Supplementary Materials for

\title{
Total Synthesis of Dapholdhamine B and Dapholdhamine B lactone
}

Lian-Dong Guo, Jieping Hou‡, Wentong Tu‡, Yan Zhang‡, Yue Zhang, Louxi Chen and Jing Xu*

Department of Chemistry and Shenzhen Grubbs Institute, Southern University of Science and Technology, China

$\ddagger$ These authors contributed equally

Correspondence to: xuj@sustech.edu.cn

General Methods

S-2

Experimental Procedures

The HPLC chromatograms of $( \pm)-5$ and $(R)-5$

Crystallographic data of compounds 16, 29 and 38

References

${ }^{1} \mathrm{H}$ and ${ }^{13} \mathrm{C}$ NMR Spectra

S-50 
General Methods. Unless otherwise mentioned, all reactions were conducted under a nitrogen atmosphere and anhydrous conditions. Tetrahydrofuran (THF) was distilled from sodium benzophenone under an argon atmosphere. Dichloromethane (DCM) was distilled from calcium hydride. Reactions were monitored by thin-layer chromatography (TLC; GF254) using plates supplied by Yantai Chemicals (China) and visualized under UV or by staining with an ethanolic solution of phosphomolybdic acid, cerium sulfate or iodine. Flash column chromatography was performed using silica gel (particle size, $0.040-0.063 \mathrm{~mm}$ ). NMR spectra were recorded on a Bruker $\mathrm{AV} 400 \mathrm{MHz}$ instrument and calibrated using residual undeuterated chloroform in $\mathrm{CDCl}_{3}(\delta \mathrm{H}=7.26 \mathrm{ppm}, \delta \mathrm{C}=77.0 \mathrm{ppm})$ or MeOD- $d_{4}(\delta \mathrm{H}=$ $3.31 \mathrm{ppm}, \delta \mathrm{C}=49.0 \mathrm{ppm}$ ) as an internal reference. The following abbreviations were used to describe signal multiplicities: s, singlet; $d$, doublet; $t$, triplet; $d$, double triplet; ddd, doublet of double doublet; ddt, doublet of double triplet; m, multiplet. High-resolution mass spectra (HRMS) were recorded on a Thermo Scientific Q Exactive Hybrid Quadrupole Orbitrap mass spectrometer. 


\section{Experimental Procedures and Characterization Data}

\section{Compound 6}<smiles>O=C1C=C(O)CCC1</smiles>

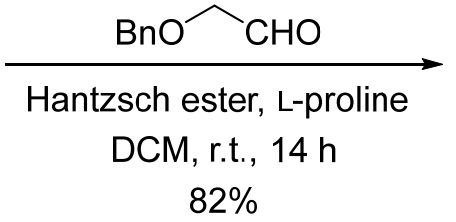<smiles>O=C1CCCC(O)=C1CCOc1ccccc1</smiles>

6

Following a modification of the literature procedure given in ref. ${ }^{1}$ : To a cooled $\left(0{ }^{\circ} \mathrm{C}\right)$ solution of benzyloxyacetaldehyde $(50.0 \mathrm{~g}, 333 \mathrm{mmol}, 1.0$ equiv) in DCM (700 mL) under argon atmosphere, were added cyclohexan-1,3-dione (74.7 g, $666 \mathrm{mmol}, 2.0$ equiv), Hantzsch ester (84.5 g, 333 mmol, 1.0 eq) and finally L-proline (3.83 g, 33.3 mmol, $0.1 \mathrm{eq})$. After being stirred for $14 \mathrm{~h}$ at room temperature, the reaction mixture was quenched with $2 \mathrm{~N} \mathrm{HCl}(400 \mathrm{~mL})$. The organic phase was separated and the aqueous phase was extracted with DCM $(3 \times 300 \mathrm{~mL})$. The combined organic phases were washed with brine, dried over anhydrous $\mathrm{Na}_{2} \mathrm{SO}_{4}$, filtered, and concentrated under reduced pressure. The residue was purified by flash chromatography on silica gel (eluent: EtOAc/PE, 1/9 to 1/3) to give compound $6(67.3 \mathrm{~g}$, yield: $82 \%)$ as a pale yellow solid.

$\mathbf{R}_{\mathbf{f}}=0.65$ (silica, EtOAc/PE $=1: 2$ );

${ }^{1} \mathbf{H}$ NMR (400 MHz, $\left.\mathrm{CDCl}_{3}\right): \delta=9.72(\mathrm{~s}, 1 \mathrm{H}), 7.40-7.27(\mathrm{~m}, 5 \mathrm{H}), 4.57(\mathrm{~s}, 2 \mathrm{H})$, $3.64-3.58(\mathrm{~m}, 2 \mathrm{H}), 2.69(\mathrm{t}, J=5.0 \mathrm{~Hz}, 2 \mathrm{H}), 2.46(\mathrm{t}, J=6.3 \mathrm{~Hz}, 2 \mathrm{H}), 2.34(\mathrm{t}, J=6.6$ $\mathrm{Hz}, 2 \mathrm{H}), 1.91(\mathrm{dt}, J=13.0,6.5 \mathrm{~Hz}, 2 \mathrm{H}) \mathrm{ppm}$;

${ }^{13} \mathrm{C}$ NMR (101 MHz, $\left.\mathrm{CDCl}_{3}\right): \delta=198.3,174.8,136.5,128.6(2 \mathrm{C}), 128.2,127.9(2 \mathrm{C})$, $113.9,73.6,71.9,36.4,29.3,22.5,20.5 \mathrm{ppm}$;

HRMS (ESI): calculated for $\left[\mathrm{C}_{15} \mathrm{H}_{18} \mathrm{O}_{3}+\mathrm{H}\right]^{+}$247.1329, found 247.1327. 


\section{Compound 5}
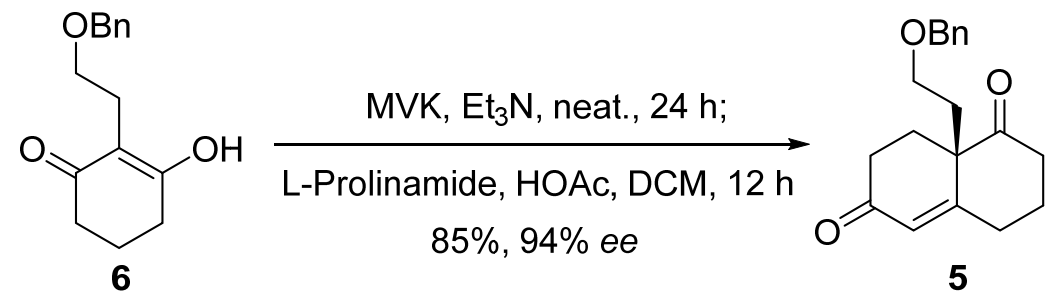

To a mixture of compound 6 (61.6 g, 250 mmol, 1.0 equiv) and MVK (41.7 mL, 500 mmol, 2.0 equiv) under argon atmosphere, was added TEA (3.48 mL, $25.0 \mathrm{mmol}, 0.1$ equiv). The initial thick suspension slowly became more fluid as the solid gradually dissolved to give a yellow/orange solution. The reaction was stirred at room temperature for $24 \mathrm{~h}$. Volatiles were removed under reduced pressure and the residue was dissolved in DCM $(650 \mathrm{~mL})$. To the resulting solution were added L-Prolinamide (28.5 g, $250 \mathrm{mmol}, 1.0$ equiv) and $\mathrm{AcOH}\left(14.3 \mathrm{~mL}, 250 \mathrm{mmol}, 1.0\right.$ equiv) at $0{ }^{\circ} \mathrm{C}$. The mixture was warmed to room temperature and stirred overnight. DCM was removed under reduced pressure and the residue was dissolved in EtOAc $(800 \mathrm{~mL})$ and $2 \mathrm{~N}$ $\mathrm{HCl}(300 \mathrm{~mL})$. The organic phase was separated and the aqueous phase was extracted with EtOAc $(3 \times 300 \mathrm{~mL})$. The combined organic phases were washed with brine, dried over anhydrous $\mathrm{Na}_{2} \mathrm{SO}_{4}$, filtered, and concentrated under reduced pressure. The residue was purified by flash chromatography on silica gel (eluent: EtOAc/PE, 1/5) to give compound 5 (63.4 g, yield: $85 \%)$ as a pale yellow oil.

$\mathbf{R f}=0.40($ silica, $\mathrm{EtOAc} / \mathrm{PE}=1: 4)$

$[\alpha]_{\mathbf{D}^{20}}=+40.5\left(c\right.$ 2.0, $\left.\mathrm{CHCl}_{3}\right)$

${ }^{1} \mathbf{H}$ NMR (400 MHz, $\left.\mathrm{CDCl}_{3}\right): \delta=7.37-7.26(\mathrm{~m}, 5 \mathrm{H}), 5.87(\mathrm{~s}, 1 \mathrm{H}), 4.40(\mathrm{~d}, J=11.5$ $\mathrm{Hz}, 1 \mathrm{H}), 4.36(\mathrm{~d}, J=11.5 \mathrm{~Hz}, 1 \mathrm{H}), 3.54(\mathrm{dt}, J=10.5,5.4 \mathrm{~Hz}, 1 \mathrm{H}), 3.41(\mathrm{dt}, J=9.5$, 8.8, 4.8 Hz, 1H), $2.84(\mathrm{dd}, J=14.3,5.7 \mathrm{~Hz}, 1 \mathrm{H}), 2.77(\mathrm{dd}, J=14.3,5.7 \mathrm{~Hz}, 1 \mathrm{H})$, 2.49-2.23 (m, 6H), 2.18-2.01 m, 3H), 1.74-1.61 (m, 1H) ppm;

${ }^{13}$ C NMR (101 MHz, $\left.\mathrm{CDCl}_{3}\right): \delta=209.9,198.1,165.8,137.6,128.3$ (2C), 127.7 (3C), $126.4,73.3,65.9,53.2,38.7,35.1,33.4,31.9,26.0,23.6 \mathrm{ppm}$;

HRMS (ESI): calculated for $\left[\mathrm{C}_{19} \mathrm{H}_{22} \mathrm{O}_{3}+\mathrm{H}\right]^{+}$299.1642, found 299.1639;

HPLC (Chiralpak AD-3, column temperature: $30^{\circ} \mathrm{C}, n$-hexane/isopropanol $=85 / 15$, flow $0.8 \mathrm{~mL} / \mathrm{min}$, detection at $254 \mathrm{~nm}$ ) retention time $=10.6 \mathrm{~min}$ (minor) and 11.4 
$\min$ (major), $e e=94 \%$.

\section{Compound 8}<smiles>O=C1C=C2CCCC(=O)C2(CCOc2ccccc2)CC1</smiles>

5
$\mathrm{CH}(\mathrm{OMe})_{3}, \mathrm{PTSA}, \mathrm{THF}, 1 \mathrm{~h}$;

7, $\mathrm{HCHO}$ aq., $7 \mathrm{~d}$ $88 \%, d r=3: 1$<smiles>[R5]N([13CH3])C[C@H]1CCC(=O)[C@]2(CCOc3ccccc3)CCC(=O)C=C12</smiles><smiles>C=CCN[AsH3+]</smiles>

7

To a solution of compound 5 (60.0 g, $201 \mathrm{mmol}, 1.0$ equiv) and $\mathrm{CH}(\mathrm{OMe})_{3}(33.0 \mathrm{~mL}$, 302 mmol, 1.5 equiv) in THF $(700 \mathrm{~mL})$ under argon atmosphere, was added p-toluenesulfonic acid monohydrate (7.65 g, $40.2 \mathrm{mmol}, 0.2$ equiv). After being stirred for $70 \mathrm{~min}$ at room tempereture, compound $7^{2}$ (71.2 g, $402 \mathrm{mmol}, 2$ equiv) and formalin $(60.0 \mathrm{~mL}, 37 \%$ aqueous, $800 \mathrm{mmol}, 4.0$ equiv) was added. The solution was stirred for 7 days at room temperature. THF was removed under reduced pressure and the residue was dissolved in MTBE $(600 \mathrm{~mL})$ and $5 \% \mathrm{NaOH}$ aq. $(200 \mathrm{~mL})$. The organic phase was separated and the aqueous phase was extracted with MTBE $(3 \times$ $200 \mathrm{~mL}$ ). The combined organic phases were washed with brine, dried over anhydrous $\mathrm{Na}_{2} \mathrm{SO}_{4}$, filtered, and concentrated under reduced pressure. The residue was purified by flash chromatography on silica gel (eluent: EtOAc/PE, 1/10 to 1/3) to give compound 8 ( $86.3 \mathrm{~g}$, yield: $88 \%$ ) as a mixture of diastereomers $(d r=3: 1$, pale yellow oil). The material was used directly for the next step without further purification.

\section{Compound 9}<smiles>C=CCN(CC)C[C@H]1CCC(=O)C2(CCOc3ccccc3)CCC(=O)C=C12</smiles>

1,3-Dimethylbarbituric acid $\mathrm{Pd}\left(\mathrm{PPh}_{3}\right)_{4}, \mathrm{DCM} / \mathrm{THF}, 4 \mathrm{~h}$;

$\mathrm{NaHCO}_{3}$ aq., $\mathrm{TsCl}, 14 \mathrm{~h}$ $82 \%, d r>6: 1$<smiles>CCCCN(C)C[C@H]1CCC(=O)C2(CCOCc3ccccc3)CCC(=O)C=C12</smiles>

To a solution of compound $8(48.8 \mathrm{~g}, 100 \mathrm{mmol}, 1.0$ equiv, $d r=3: 1)$ and 1,3-Dimethylbarbituric acid (34.4 g, $220 \mathrm{mmol}, 2.2$ equiv) in a mixture of DCM (500 
$\mathrm{mL})$ and THF $(100 \mathrm{~mL})$ under argon atmosphere, was added $\mathrm{Pd}\left(\mathrm{PPh}_{3}\right)_{4}(5.78 \mathrm{~g}, 5.00$ mmol, 0.05 equiv). After being stirred for $4 \mathrm{~h}$ at room tempereture, saturated aqueous $\mathrm{NaHCO}_{3}(500 \mathrm{~mL})$ and $\mathrm{TsCl}(38.1 \mathrm{~g}, 200 \mathrm{mmol}, 2.0$ equiv) was added. The solution was stirred for $14 \mathrm{~h}$ at room temperature. The organic phase was separated and the aqueous phase was extracted with DCM $(3 \times 200 \mathrm{~mL})$. The combined organic phases were washed with brine, dried over anhydrous $\mathrm{Na}_{2} \mathrm{SO}_{4}$, filtered, and concentrated under reduced pressure. The residue was purified by flash chromatography on silica gel (eluent: EtOAc/PE, $1 / 5$ to $1 / 3)$ to give compound $9(49.3 \mathrm{~g}$, yield: $82 \%)$ as a mixture of diastereomers $(d r>6: 1)$. The material was used directly for the next step without further purification.

A small amount of material was treated with DBU to get a pure compound $\mathbf{9}(d r>$ 50:1): To a solution of compound 9 (603 $\mathrm{mg}, 1.00 \mathrm{mmol}, 1.0$ equiv, $d r>6: 1)$ in DCM $(10 \mathrm{~mL})$ under argon atmosphere, was added DBU $(0.0299 \mathrm{~mL}, 0.200 \mathrm{mmol}, 0.20$ equiv). After being stirred for $3 \mathrm{~h}$ at room tempereture, $2 N \mathrm{HCl}(10 \mathrm{~mL})$ was added. The organic phase was separated and the aqueous phase was extracted with EtOAc (3 $\times 20 \mathrm{~mL}$ ). The combined organic phases were washed with brine, dried over anhydrous $\mathrm{Na}_{2} \mathrm{SO}_{4}$, filtered, and concentrated under reduced pressure. The residue was purified by flash chromatography on silica gel (eluent: EtOAc/PE, 1/3) to give compound 9 (573 mg, yield: $95 \%, d r>50: 1)$ as a pale yellow oil.

$\mathbf{R f}=0.55($ silica, $\mathrm{EtOAc} / \mathrm{PE}=1: 2)$;

$[\alpha]_{\mathbf{D}}{ }^{\mathbf{2 0}}=-1.0\left(c 1.0, \mathrm{CHCl}_{3}\right) ;$

${ }^{1} \mathbf{H}$ NMR (400 MHz, $\left.\mathrm{CDCl}_{3}\right): \delta=7.71(\mathrm{~d}, J=7.9 \mathrm{~Hz}, 2 \mathrm{H}), 7.39-7.19(\mathrm{~m}, 9 \mathrm{H}), 6.84(\mathrm{~d}$, $J=8.2 \mathrm{~Hz}, 2 \mathrm{H}), 5.54(\mathrm{~s}, 1 \mathrm{H}), 4.50(\mathrm{~d}, J=14.0 \mathrm{~Hz}, 1 \mathrm{H}), 4.36(\mathrm{~d}, J=11.6 \mathrm{~Hz}, 1 \mathrm{H})$, $4.32(\mathrm{~d}, J=11.6 \mathrm{~Hz}, 1 \mathrm{H}), 3.70$ (s, 3H), 3.70-3.65 (m, 1H), 3.43-3.25 (m, 3H), 2.95 $(\mathrm{dd}, J=13.5,3.6 \mathrm{~Hz}, 1 \mathrm{H}), 2.52-2.42(\mathrm{~m}, 4 \mathrm{H}), 2.39-2.20(\mathrm{~m}, 5 \mathrm{H}), 2.13(\mathrm{dt}, J=14.5$, $5.0 \mathrm{~Hz}, 1 \mathrm{H}), 2.09-1.97(\mathrm{~m}, 1 \mathrm{H}), 1.77$ (t, $J=5.9 \mathrm{~Hz}, 2 \mathrm{H}), 1.21-1.06(\mathrm{~m}, 1 \mathrm{H}) \mathrm{ppm}$;

${ }^{13}$ C NMR (101 MHz, $\left.\mathrm{CDCl}_{3}\right): \delta=209.3,197.9,165.7,159.5,143.7,137.6,135.1$, 129.9 (4C), 128.2 (2C), 128.1, 127.7 (2C), 127.6, 127.1 (2C), 123.0, 114.0 (2C), 73.1, 65.7, 55.1, 54.1, 52.9, 51.3, 37.4, 36.9, 36.0, 33.1, 26.7, 26.2, 21.4 ppm;

HRMS (ESI): calculated for $\left[\mathrm{C}_{35} \mathrm{H}_{39} \mathrm{NO}_{6} \mathrm{~S}+\mathrm{H}\right]^{+}$602.2571, found 602.2570. 


\section{Compound 10}

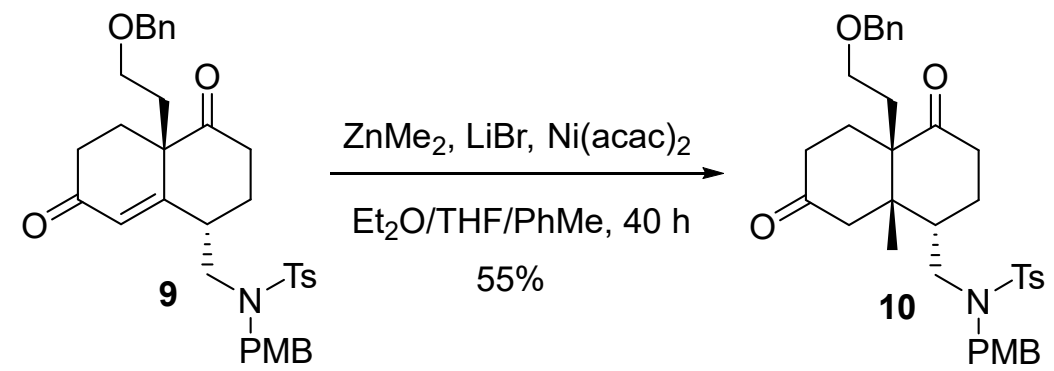

Using a glovebox, a flask was charged with dry $\operatorname{LiBr}(17.4$ g, 200.0 mmol, 10 equiv) and $\mathrm{Ni}(\mathrm{acac})_{2}(0.514 \mathrm{~g}, 2.00 \mathrm{mmol}, 0.10$ equiv) and then flushed with argon, and dry $\mathrm{Et}_{2} \mathrm{O}(40 \mathrm{~mL})$ was added. Dimethylzinc $(100 \mathrm{~mL}, 100 \mathrm{mmol}, 1.0 \mathrm{M}$ in toluene, 5.0 equiv) was added dropwise to this stirred suspension at $0{ }^{\circ} \mathrm{C}$ under Ar. The brown heterogeneous mixture was stirred at $0{ }^{\circ} \mathrm{C}$ for $30 \mathrm{~min}$, and then a solution of enone 9 (12.0 g, $20.0 \mathrm{mmol}, 1.0$ equiv, $d r>6: 1)$ in THF $(20 \mathrm{~mL})$ was added over $30 \mathrm{~min}$. The reaction was allowed to warm to room temperature and stirred at room temperature for $40 \mathrm{~h}$. This mixture was cooled to $0{ }^{\circ} \mathrm{C}$ and quenched with saturated aqueous $\mathrm{NH}_{4} \mathrm{Cl}(20 \mathrm{~mL}) .2 \mathrm{~N} \mathrm{HCl}(100 \mathrm{~mL})$ was then added, and the organic layer was separated. The aqueous phase was extracted with EtOAc $(3 \times 200 \mathrm{~mL})$. The combined organic phases were washed with brine, dried over anhydrous $\mathrm{Na}_{2} \mathrm{SO}_{4}$, filtered, and concentrated under reduced pressure. The residue was purified by flash chromatography on silica gel (eluent: EtOAc/PE, $1 / 5$ to $1 / 3$ ) to give compound $\mathbf{1 0}$ $(6.80 \mathrm{~g}$, yield: $55 \%)$ as a colorless oil.

$\mathbf{R f}=0.58$ (silica, $\mathrm{EtOAc} / \mathrm{PE}=1: 2)$

$[\alpha]_{\mathbf{D}}^{\mathbf{2 0}}=-20.0\left(c\right.$ 1.0, $\left.\mathrm{CHCl}_{3}\right)$;

${ }^{1} \mathbf{H}$ NMR (400 MHz, $\left.\mathrm{CDCl}_{3}\right): \delta=7.68(\mathrm{~d}, J=8.0 \mathrm{~Hz}, 2 \mathrm{H}), 7.42-7.31(\mathrm{~m}, 4 \mathrm{H})$, $7.31-7.25(\mathrm{~m}, 3 \mathrm{H}), 7.22(\mathrm{~d}, J=8.3 \mathrm{~Hz}, 2 \mathrm{H}), 6.86(\mathrm{~d}, J=8.3 \mathrm{~Hz}, 2 \mathrm{H}), 4.51(\mathrm{~d}, J=$ $14.0 \mathrm{~Hz}, 1 \mathrm{H}), 4.37(\mathrm{~d}, J=11.9 \mathrm{~Hz}, 1 \mathrm{H}), 4.31(\mathrm{~d}, J=11.9 \mathrm{~Hz}, 1 \mathrm{H}), 3.82(\mathrm{~d}, J=14.0$ $\mathrm{Hz}, 1 \mathrm{H}), 3.69$ (s, 3H), 3.36-3.29 (m, 1H), $3.13(\mathrm{dt}, J=9.4,7.1 \mathrm{~Hz}, 1 \mathrm{H}), 3.02(\mathrm{dd}, J=$ 13.6, 11.2 Hz, 1H), 2.85-2.76 (m, 1H), 2.65-2.48 (m, 2H), 2.45 (s, 3H), 2.35 (dt, $J=$ 14.1, 7.1 Hz, 1H), 2.27-2.19 (m, 1H), 2.19-2.09 (m, 3H), 1.99-1.82 (m, 2H), 1.70 (br $\mathrm{d}, J=13.2,1 \mathrm{H}), 1.46-1.28(\mathrm{~m}, 3 \mathrm{H}), 0.84(\mathrm{~s}, 3 \mathrm{H}) \mathrm{ppm}$;

${ }^{13} \mathrm{C}$ NMR $\left(101 \mathrm{MHz}, \mathrm{CDCl}_{3}\right): \delta=213.1,211.3,159.6,143.6,137.8,135.5,130.1$ 
(2C), 129.9 (2C), 128.3 (2C), 128.1, 127.6, 127.5 (2C), 127.1 (2C), 114.0 (2C), 73.2, 65.7, 55.1, 54.2, 53.3, 50.0, 47.4, 45.1, 38.5, 38.1, 37.9, 34.7, 28.0, 26.0, 21.5, 20.4 ppm;

HRMS (ESI): calculated for $\left[\mathrm{C}_{36} \mathrm{H}_{43} \mathrm{NO}_{6} \mathrm{~S}+\mathrm{H}\right]^{+}$618.2884, found 618.2881.

\section{Compound 12}
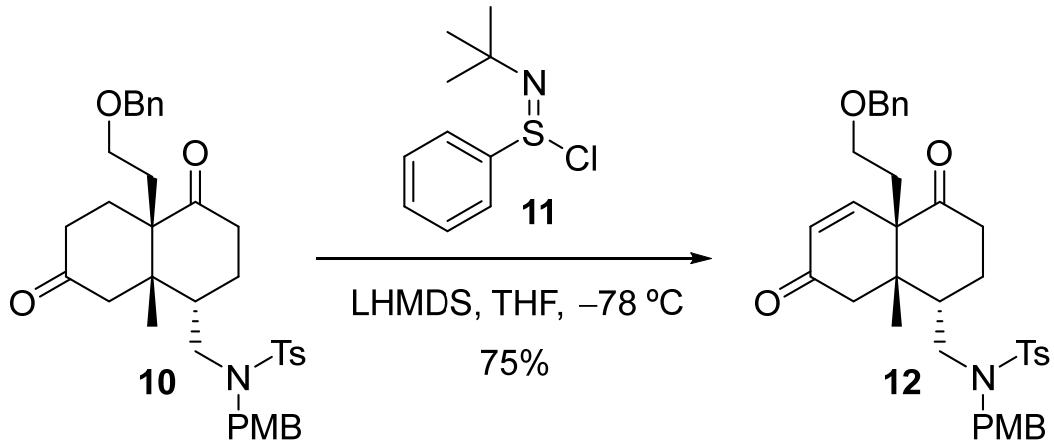

To a solution of ketone $\mathbf{1 0}$ (15.5 g, $25.1 \mathrm{mmol}, 1.0$ equiv) in THF (250 ml) was added a solution of 1.0 M LHMDS (26.3 mL, $26.3 \mathrm{mmol}, 1.05$ equiv) in THF at $-78{ }^{\circ} \mathrm{C}$ under argon. After being stirred for $1 \mathrm{~h}$ at $-50{ }^{\circ} \mathrm{C}$, the reaction mixture was recooled to $-78{ }^{\circ} \mathrm{C}$, and $N$-tert-butylbenzenesulfinimidoyl chloride $(\mathbf{1 1})^{3}$ (8.12 g, $37.7 \mathrm{mmol}$, 1.5 equiv) in THF ( $25 \mathrm{~mL}$ ) was added. The resulting yellow mixture was stirred at this temperature for $1 \mathrm{~h}$, then quenched with saturated aqueous $\mathrm{NH}_{4} \mathrm{Cl}(300 \mathrm{~mL})$ and warmed to room temperature. THF was removed under reduced pressure, the aqueous phase was extracted with DCM $(3 \times 250 \mathrm{~mL})$. The combined organic phases were washed with brine, dried over anhydrous $\mathrm{Na}_{2} \mathrm{SO}_{4}$, filtered, and concentrated under reduced pressure. The residue was purified by flash chromatography on silica gel (eluent: EtOAc/PE, 1/10 to 1/3) to give enone 12 (11.6 g, yield: $75 \%$ ) as a pale yellow foam.

$\mathbf{R f}=0.53($ silica, $\mathrm{EtOAc} / \mathrm{PE}=1: 2)$

$[\alpha]_{\mathbf{D}^{20}}=-4.3\left(\right.$ c $\left.1.0, \mathrm{CHCl}_{3}\right) ;$

${ }^{1} \mathbf{H}$ NMR (400 MHz, $\left.\mathrm{CDCl}_{3}\right): \delta=7.70(\mathrm{~d}, J=7.9 \mathrm{~Hz}, 2 \mathrm{H}), 7.39-7.25(\mathrm{~m}, 7 \mathrm{H}), 7.23(\mathrm{~d}$, $J=8.5 \mathrm{~Hz}, 2 \mathrm{H}), 6.90-6.83(\mathrm{~m}, 2 \mathrm{H}), 6.77(\mathrm{~d}, J=10.4 \mathrm{~Hz}, 1 \mathrm{H}), 6.04(\mathrm{~d}, J=10.4 \mathrm{~Hz}$, 1H), 4.47 (d, $J=13.9 \mathrm{~Hz}, 1 \mathrm{H}), 4.42(\mathrm{~d}, J=11.7 \mathrm{~Hz}, 1 \mathrm{H}), 4.38(\mathrm{~d}, J=11.7 \mathrm{~Hz}, 1 \mathrm{H})$, $3.88(\mathrm{~d}, J=14.0 \mathrm{~Hz}, 1 \mathrm{H}), 3.72(\mathrm{~s}, 3 \mathrm{H}), 3.47$ (dt, $J=10.8,5.6 \mathrm{~Hz}, 1 \mathrm{H}), 3.40-3.32(\mathrm{~m}$, 
1H), $3.04(\mathrm{dd}, J=13.6,11.2 \mathrm{~Hz}, 1 \mathrm{H}), 2.83(\mathrm{dd}, J=13.7,2.9 \mathrm{~Hz}, 1 \mathrm{H}), 2.46(\mathrm{~s}, 3 \mathrm{H})$, 2.41-2.30 (m, 1H), 2.27-2.18 (m, 2H), 2.17-2.10 (m, 1H), 1.98-1.78 (m, 3H), $1.57-1.49(\mathrm{~m}, 1 \mathrm{H}), 1.31-1.17(\mathrm{~m}, 1 \mathrm{H}), 0.89$ (s, 3H) ppm;

${ }^{13} \mathbf{C}$ NMR (101 MHz, $\left.\mathrm{CDCl}_{3}\right): \delta=212.3,197.9,159.7,149.8,143.7,137.6,135.4$, 130.2 (2C), 130.0 (2C), 128.4 (2C), 128.2, 128.1, 127.8, 127.7 (2C), 127.3 (2C), 114.1 (2C), 73.5, 65.4, 57.4, 55.2, 54.4, 49.7, 45.4, 42.5, 39.3, 38.6, 32.0, 25.8, 21.5, 20.7 ppm;

HRMS (ESI): calculated for $\left[\mathrm{C}_{36} \mathrm{H}_{41} \mathrm{NO}_{6} \mathrm{~S}+\mathrm{H}\right]^{+}$616.2727, found 616.2726.

\section{Compound ( \pm )-13}<smiles>CN(C)C[C@H]1CCC(=O)[C@]2(CCOc3ccccc3)C=CC(=O)C[C@@]12C</smiles>

$( \pm)-12$

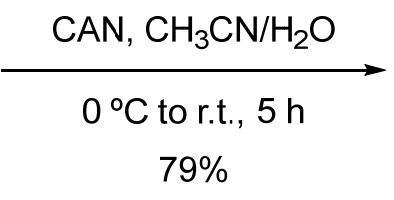

$79 \%$

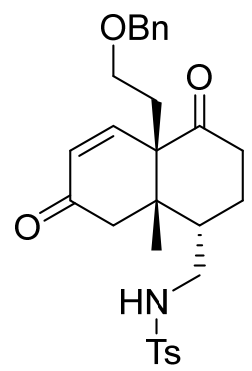

$( \pm)-13$

To a cooled $\left(0{ }^{\circ} \mathrm{C}\right)$ solution of enone $( \pm)-12(616 \mathrm{mg}, 1.00 \mathrm{mmol}, 1.0$ equiv) in a mixture of $\mathrm{CH}_{3} \mathrm{CN}(28 \mathrm{~mL})$ and $\mathrm{H}_{2} \mathrm{O}(7 \mathrm{~mL})$, was added CAN (2.20 g, $4.00 \mathrm{mmol}, 4.0$ equiv). After being stirred for $1 \mathrm{~h}$ at $0{ }^{\circ} \mathrm{C}$, the solution was warmed to room temperature and stirred for $4 \mathrm{~h}$. The crude mixture was partitioned between EtOAc $(60 \mathrm{~mL})$ and $\mathrm{H}_{2} \mathrm{O}(20 \mathrm{~mL})$, and the aqueous phase was extracted with EtOAc $(3 \times 30$ $\mathrm{mL})$. The combined organic phases were washed with $\mathrm{H}_{2} \mathrm{O}(3 \times 30 \mathrm{~mL})$, saturated aqueous $\mathrm{NaHCO}_{3}(30 \mathrm{~mL})$ and brine $(30 \mathrm{~mL})$, dried over anhydrous $\mathrm{Na}_{2} \mathrm{SO}_{4}$, filtered, and concentrated under reduced pressure. The residue was purified by flash chromatography on silica gel (eluent: EtOAc/PE, 1/4 to 2/3) to give compound ( \pm )-13 (393 mg, yield: 79\%) as a white foam.

$\mathbf{R f}=0.50($ silica, $\mathrm{EtOAc} / \mathrm{PE}=1: 1)$

${ }^{1} \mathbf{H}$ NMR (400 MHz, $\left.\mathrm{CDCl}_{3}\right): \delta=7.71(\mathrm{~d}, J=7.8 \mathrm{~Hz}, 2 \mathrm{H}), 7.37-7.24(\mathrm{~m}, 7 \mathrm{H}), 6.82(\mathrm{~d}$, $J=10.4 \mathrm{~Hz}, 1 \mathrm{H}), 6.07$ (d, $J=10.4 \mathrm{~Hz}, 1 \mathrm{H}), 5.32$ (t, $J=6.4 \mathrm{~Hz}, 1 \mathrm{H}), 4.40$ (d, $J=11.7$ $\mathrm{Hz}, 1 \mathrm{H}), 4.36$ (d, $J=11.7 \mathrm{~Hz}, 1 \mathrm{H}), 3.61-3.53$ (m, 1H), 3.52-3.44 (m, 1H), 3.06 (dd, $J$ 
$=12.3,5.4 \mathrm{~Hz}, 1 \mathrm{H}), 2.81-2.67(\mathrm{~m}, 1 \mathrm{H}), 2.61-2.46(\mathrm{~m}, 1 \mathrm{H}), 2.40(\mathrm{~s}, 3 \mathrm{H}), 2.37-2.16$ (m, 5H), 1.91-1.81 (m, 1H), $1.71(\mathrm{dt}, J=14.7,5.0 \mathrm{~Hz}, 1 \mathrm{H}), 1.49-1.37$ (m, 1H), 1.04 $(\mathrm{s}, 3 \mathrm{H}) \mathrm{ppm}$;

${ }^{13}$ C NMR (101 MHz, $\left.\mathrm{CDCl}_{3}\right): \delta=212.1,197.7,149.8,143.8,137.4,136.5,129.9$ (2C), 128.4 (2C), 128.1, 127.8 (3C), 126.9 (2C), 73.5, 65.4, 57.3, 45.6, 43.5, 42.6, 41.5, 38.4, 32.1, 26.3, 21.4, $21.0 \mathrm{ppm}$;

HRMS (ESI): calculated for $\left[\mathrm{C}_{28} \mathrm{H}_{33} \mathrm{NO}_{5} \mathrm{~S}+\mathrm{Na}\right]^{+}$518.1972, found 518.1967.

\section{Compound ( \pm )-15}<smiles>C[C@]12CC(=O)C=C[C@]1(CCOCc1ccccc1)C(=O)CC[C@@H]2CN[As]</smiles>

$( \pm)-13$

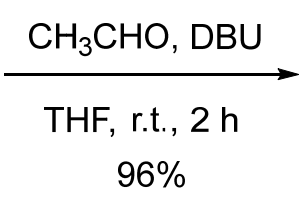

$96 \%$

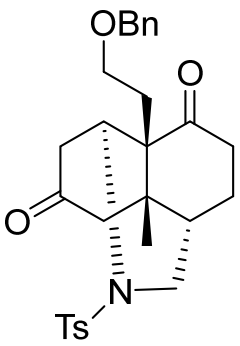

$( \pm)-15$

To a solution of compound $( \pm)-\mathbf{1 3}(99.2 \mathrm{mg}, 0.200 \mathrm{mmol}, 1.0$ equiv) in THF (2 $\mathrm{mL})$ under argon atmosphere, were added $\mathrm{CH}_{3} \mathrm{CHO}(0.400 \mathrm{~mL}, 5 \mathrm{M}$ in THF, 2.00 mmol, 10.0 equiv) and DBU (0.0598 mL, $0.400 \mathrm{mmol}, 2.0$ equiv). After being stirred for $2 \mathrm{~h}$ at room temperature, $0.5 \mathrm{~N} \mathrm{HCl}(5 \mathrm{~mL})$ and EtOAc $(15 \mathrm{~mL})$ were added. The organic phase was separated and the aqueous phase was extracted with EtOAc $(3 \times 10$ $\mathrm{mL}$ ). The combined organic phases were washed with brine, dried over anhydrous $\mathrm{Na}_{2} \mathrm{SO}_{4}$, filtered, and concentrated under reduced pressure. The residue was purified by flash chromatography on silica gel (eluent: EtOAc/PE, 2/5) to give compound (士)-15 (95.6 mg, yield: 96\%) as a colorless oil.

$\mathbf{R f}=0.60($ silica, $\mathrm{EtOAc} / \mathrm{PE}=1: 2)$

${ }^{1} \mathbf{H}$ NMR $\left(400 \mathrm{MHz}, \mathrm{CDCl}_{3}\right): \delta=7.53(\mathrm{~d}, J=7.9 \mathrm{~Hz}, 2 \mathrm{H}), 7.37-7.21(\mathrm{~m}, 7 \mathrm{H})$, 4.53-4.48 (m, 1H), $4.48(\mathrm{~d}, J=12.2 \mathrm{~Hz}, 1 \mathrm{H}), 4.44(\mathrm{~d}, J=12.2 \mathrm{~Hz}, 1 \mathrm{H}), 3.97-3.80(\mathrm{~m}$, $3 \mathrm{H}), 3.12(\mathrm{~d}, J=13.6 \mathrm{~Hz}, 1 \mathrm{H}), 3.01(\mathrm{dt}, J=18.1,10.4 \mathrm{~Hz}, 1 \mathrm{H}), 2.80(\mathrm{dd}, J=18.3,4.3$ Hz, 1H), 2.45 (dd, $J=18.1,8.5 \mathrm{~Hz}, 1 \mathrm{H}), 2.40$ (s, 3H), 2.38-2.27 (m, 2H), 2.24-2.10 (m, 2H), 1.98-1.85 (m, 2H), 1.69-1.58 (m, 2H), 0.96 (s, 3H) ppm;

${ }^{13}$ C NMR (101 MHz, $\left.\mathrm{CDCl}_{3}\right): \delta=212.9,207.7,143.9,138.0,136.4,130.0$ (2C), 
128.4 (2C), 127.7 (2C), 127.6, 126.7 (2C), 73.3, 68.0, 54.8, 54.5, 50.6, 45.9, 40.7, 40.6, 39.9, 38.1, 29.0, 26.2, 24.0, 21.5 ppm;

HRMS (ESI): calculated for $\left[\mathrm{C}_{28} \mathrm{H}_{33} \mathrm{NO}_{5} \mathrm{~S}+\mathrm{Na}\right]^{+}$518.1972, found 518.1968.

\section{Compound 16}<smiles>CCCCN([13F])C[C@@H]1CCC(=O)[C@]2(CCOCc3ccccc3)C=CC(=O)C[C@]12C</smiles>

12
$\mathrm{H}_{2} \mathrm{O}_{2}, \mathrm{NaOH}, \mathrm{THF} / \mathrm{MeOH} / \mathrm{H}_{2} \mathrm{O}$ $0{ }^{\circ} \mathrm{C}, 2 \mathrm{~h}$ $95 \%$

To a cooled $\left(0^{\circ} \mathrm{C}\right)$ solution of enone $12(6.16 \mathrm{~g}, 10.0 \mathrm{mmol}, 1.0$ equiv) in a mixture of $\mathrm{MeOH}(100 \mathrm{~mL})$ and $\mathrm{THF}(100 \mathrm{~mL})$, were added 30\% aqueous hydrogen peroxide $(5.12 \mathrm{~mL}, 50.0 \mathrm{mmol}, 5.0$ equiv) and $15 \%$ aqueous sodium hydroxide $(2.67 \mathrm{~mL}$, $10.0 \mathrm{mmol}, 1.0$ equiv). After being stirred for $2 \mathrm{~h}$ at $0{ }^{\circ} \mathrm{C}$, the reaction mixture was quenched with saturated aqueous $\mathrm{Na}_{2} \mathrm{~S}_{2} \mathrm{O}_{3}(50 \mathrm{~mL})$. Volatiles were removed under reduced pressure and DCM $(200 \mathrm{~mL})$ was added. The organic phase was separated and the aqueous phase was extracted with DCM $(2 \times 150 \mathrm{~mL})$. The combined organic phases were washed with brine, dried over anhydrous $\mathrm{Na}_{2} \mathrm{SO}_{4}$, filtered, and concentrated under reduced pressure. The residue was purified by flash chromatography on silica gel (eluent: EtOAc/DCM, 1/50) to give compound 16 (6.01 g, yield: 95\%) as a white solid.

$\mathbf{R f}=0.53($ silica, $\mathrm{EtOAc} / \mathrm{PE}=1: 2)$

$[\alpha]_{\mathbf{D}}{ }^{\mathbf{2 0}}=-7.0\left(c 1.0, \mathrm{CHCl}_{3}\right)$;

mp: $85-90{ }^{\circ} \mathrm{C}$;

${ }^{1} \mathbf{H}$ NMR $\left(400 \mathrm{MHz}, \mathrm{CDCl}_{3}\right): \delta=7.68(\mathrm{~d}, J=7.8 \mathrm{~Hz}, 2 \mathrm{H}), 7.40-7.26(\mathrm{~m}, 7 \mathrm{H}), 7.20(\mathrm{~d}$, $J=8.4 \mathrm{~Hz}, 2 \mathrm{H}), 6.84(\mathrm{~d}, J=7.9 \mathrm{~Hz}, 2 \mathrm{H}), 4.49$ (d, $J=14.0 \mathrm{~Hz}, 1 \mathrm{H}), 4.39$ (d, $J=11.5$ $\mathrm{Hz}, 1 \mathrm{H}), 4.33$ (d, $J=11.5 \mathrm{~Hz}, 1 \mathrm{H}), 3.82$ (d, $J=14.1 \mathrm{~Hz}, 1 \mathrm{H}), 3.71(\mathrm{~s}, 3 \mathrm{H}), 3.49-3.41$ (m, 2H), 3.28-3.19 (m, 2H), $3.04(\mathrm{dd}, J=13.6,11.2 \mathrm{~Hz}, 1 \mathrm{H}), 2.78(\mathrm{dd}, J=13.6,2.8$ $\mathrm{Hz}, 1 \mathrm{H}), 2.55$ (d, $J=12.9 \mathrm{~Hz}, 1 \mathrm{H}), 2.46$ (s, 3H), 2.38-2.28 (m, 1H), 2.10-1.97 (m, 2H), 1.75-1.57 (m, 3H), $1.48(\mathrm{~d}, J=12.9 \mathrm{~Hz}, 1 \mathrm{H}), 1.21-1.09(\mathrm{~m}, 1 \mathrm{H}), 0.77(\mathrm{~s}, 3 \mathrm{H})$ 
ppm;

${ }^{13}$ C NMR (101 MHz, $\left.\mathrm{CDCl}_{3}\right): \delta=209.1,205.8,159.7,143.8,137.7,135.5,130.1$ (2C), 130.0 (2C), 128.4 (2C), 128.2, 127.9 (2C), 127.8, 127.2 (2C), 114.1 (2C), 73.5, $65.5,60.6,55.2,54.6,54.6,54.3,49.6,47.2,40.2,39.5$ (2C), 32.1, 23.4, 21.5, 21.5 ppm;

HRMS (ESI): calculated for $\left[\mathrm{C}_{36} \mathrm{H}_{41} \mathrm{NO}_{7} \mathrm{~S}+\mathrm{Na}\right]^{+}$654.2496, found 654.2488.

\section{Compound 17}

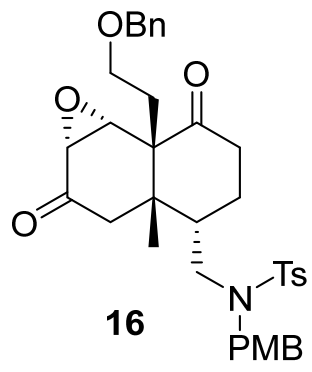

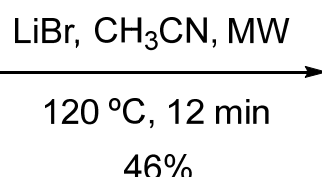

$46 \%$

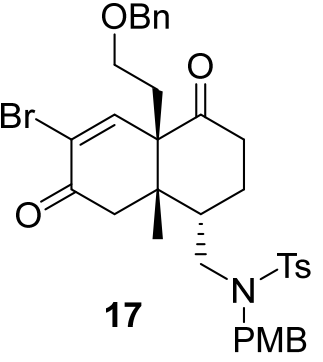

Using a glovebox, a sealed reaction glass vial containing a stirring bar was charged with dry $\operatorname{LiBr}(1.74 \mathrm{~g}, 20.0 \mathrm{mmol}, 10$ equiv) and then flushed with argon, and a solution of compound 16 (1.26 g, $2.00 \mathrm{mmol}, 1.0$ equiv) in dry $\mathrm{CH}_{3} \mathrm{CN}$ (15 mL) was added. The mixture was irradiated (microwave) for $12 \mathrm{~min}$ at a pre-selected temperature of $120^{\circ} \mathrm{C}$. After the reaction, the vial was cooled to room temperature by gas jet cooling. The crude mixture was partitioned between EtOAc $(20 \mathrm{~mL})$ and $\mathrm{H}_{2} \mathrm{O}$ $(20 \mathrm{~mL})$, and the aqueous phase was extracted with EtOAc $(3 \times 20 \mathrm{~mL})$. The combined organic phases were washed with brine, dried over anhydrous $\mathrm{Na}_{2} \mathrm{SO}_{4}$, filtered, and concentrated under reduced pressure. The residue was purified by flash chromatography on silica gel (eluent: EtOAc/PE, 1/10 to 1/5) to give compound $\mathbf{1 7}$ (639 mg, yield: 46\%) as a white solid.

$\mathbf{R f}=0.70($ silica, $\mathrm{EtOAc} / \mathrm{PE}=1: 2)$;

$[\alpha]_{\mathbf{D}}^{\mathbf{2 0}}=-32.0\left(c 1.0, \mathrm{CHCl}_{3}\right)$;

${ }^{1} \mathbf{H}$ NMR $\left(400 \mathrm{MHz}, \mathrm{CDCl}_{3}\right): \delta=7.69(\mathrm{~d}, J=8.2 \mathrm{~Hz}, 2 \mathrm{H}), 7.39-7.32(\mathrm{~m}, 4 \mathrm{H})$, $7.32-7.24(\mathrm{~m}, 4 \mathrm{H}), 7.22(\mathrm{~d}, J=8.5 \mathrm{~Hz}, 2 \mathrm{H}), 6.86(\mathrm{~d}, J=8.5 \mathrm{~Hz}, 2 \mathrm{H}), 4.47(\mathrm{~d}, J=$ $13.9 \mathrm{~Hz}, 1 \mathrm{H}), 4.37$ (s, 2H), 3.85 (d, $J=13.9 \mathrm{~Hz}, 1 \mathrm{H}), 3.71(\mathrm{~s}, 3 \mathrm{H}), 3.46$ (dt, $J=10.3$, $5.3 \mathrm{~Hz}, 1 \mathrm{H}), 3.42-3.34(\mathrm{~m}, 1 \mathrm{H}), 3.10-2.95(\mathrm{~m}, 1 \mathrm{H}), 2.83(\mathrm{dd}, J=13.6,2.4 \mathrm{~Hz}, 1 \mathrm{H})$, 
$2.45(\mathrm{~s}, 3 \mathrm{H}), 2.41-2.29(\mathrm{~m}, 2 \mathrm{H}), 2.24-2.05(\mathrm{~m}, 3 \mathrm{H}), 1.92-1.78(\mathrm{~m}, 2 \mathrm{H}), 1.57(\mathrm{dt}, J=$ 14.6, 5.1 Hz, 1H), 1.28-1.13 (m, 1H), 0.90 (s, 3H) ppm;

${ }^{13}$ C NMR (101 MHz, $\left.\mathrm{CDCl}_{3}\right): \delta=211.0,190.0,159.6,150.4,143.7,137.4,135.2$, 130.1 (2C), 129.9 (2C), 128.3 (2C), 128.0, 127.7, 127.6 (2C), 127.1 (2C), 121.6, 114.0 (2C), 73.4, 65.2, 60.6, 55.1, 54.3, 49.5, 45.7, 42.5, 39.2, 38.5, 31.7, 25.7, 21.4, 20.7 ppm;

HRMS (ESI): calculated for $\left[\mathrm{C}_{36} \mathrm{H}_{40} \mathrm{BrNO}_{6} \mathrm{~S}+\mathrm{Na}\right]^{+}$716.1652, found 716.1645.

\section{Compound 19}

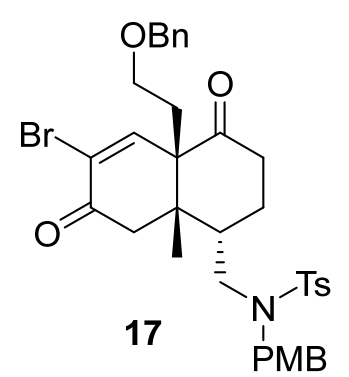
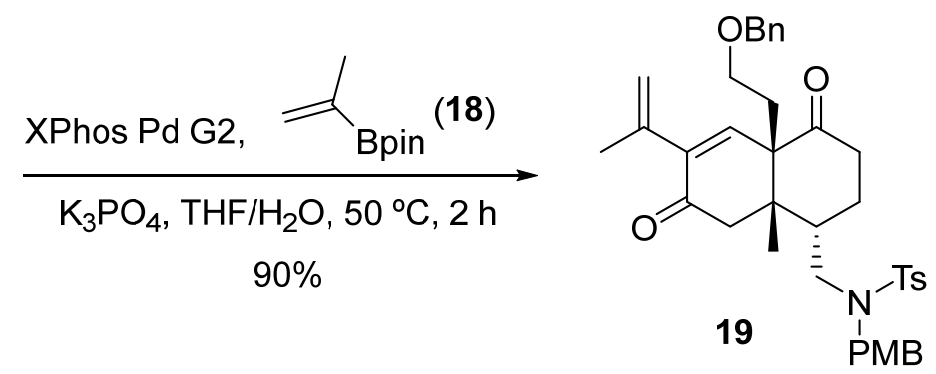

To a solution of compound 17 (13.89 g, $20.0 \mathrm{mmol}$, 1.0 equiv) and Xphos Pd G2 (0.944 g, $1.20 \mathrm{mmol}, 0.06$ equiv) in THF(100 mL) under argon atmosphere, were added $\mathrm{K}_{3} \mathrm{PO}_{4}$ in degassed $\mathrm{H}_{2} \mathrm{O}(60.0 \mathrm{~mL}, 60.0 \mathrm{mmol}, 1.0 \mathrm{M}, 3.0$ equiv) and isopropenylboronic acid pinacol ester (18) $(7.52 \mathrm{~mL}, 40.0 \mathrm{mmol}, 2.0$ equiv). The reaction was allowed to stir for $2 \mathrm{~h}$ at $50{ }^{\circ} \mathrm{C}$. The mixture was cooled to room temperature, and the organic phase was separated. The aqueous phase was extracted with EtOAc $(3 \times 100 \mathrm{~mL})$. The combined organic phases were washed with brine, dried over anhydrous $\mathrm{Na}_{2} \mathrm{SO}_{4}$, filtered, and concentrated under reduced pressure. The residue was purified by flash chromatography on silica gel (eluent: EtOAc/PE, 1/20 to 1/5) to give compound 19 (11.8 g, yield: 90\%) as a white foam.

$\mathbf{R f}=0.73($ silica, $\mathrm{EtOAc} / \mathrm{PE}=1: 2)$;

$[\alpha]_{\mathbf{D}^{20}}=-39.0\left(\right.$ c 1.0, $\left.\mathrm{CHCl}_{3}\right)$;

${ }^{1} \mathbf{H}$ NMR (400 MHz, $\left.\mathrm{CDCl}_{3}\right): \delta=7.73-7.67$ (m, 2H), 7.38-7.32 (m, 4H), 7.32-7.26 (m, 3H), 7.24-7.19 (m, 2H), 6.89-6.83 (m, 2H), 6.61 (s, 1H), 5.15 (dd, J=2.2, $1.0 \mathrm{~Hz}$, 1H), 5.07-5.01 (m, 1H), $4.46(\mathrm{~d}, J=14.0 \mathrm{~Hz}, 1 \mathrm{H}), 4.40(\mathrm{~d}, J=11.6 \mathrm{~Hz}, 1 \mathrm{H}), 4.36(\mathrm{~d}$, $J=11.7 \mathrm{~Hz}, 1 \mathrm{H}), 3.88(\mathrm{~d}, J=14.0 \mathrm{~Hz}, 1 \mathrm{H}), 3.72(\mathrm{~s}, 3 \mathrm{H}), 3.54-3.44(\mathrm{~m}, 1 \mathrm{H})$, 
3.43-3.34 (m, 1H), $3.04(\mathrm{dd}, J=13.7,11.2 \mathrm{~Hz}, 1 \mathrm{H}), 2.84(\mathrm{dd}, J=13.7,2.9 \mathrm{~Hz}, 1 \mathrm{H})$, 2.46 (s, 3H), 2.40-2.24 (m, 2H), 2.20 (ddd, $J=14.1,4.6,2.4 \mathrm{~Hz}, 1 \mathrm{H}), 2.15-2.07$ (m, 1H), $1.96-1.80(\mathrm{~m}, 6 \mathrm{H}), 1.55(\mathrm{dt}, J=14.5,5.6 \mathrm{~Hz}, 1 \mathrm{H}), 1.29-1.15(\mathrm{~m}, 1 \mathrm{H}), 0.88(\mathrm{~s}$, 3H) ppm;

${ }^{13}$ C NMR (101 MHz, $\left.\mathrm{CDCl}_{3}\right): \delta=212.3,196.9,159.7,145.0,143.7,140.3,139.7$, 137.6, 135.4, 130.2 (2C), 129.9 (2C), 128.4 (2C), 128.1, 127.7, 127.7 (2C), 127.2 (2C), 116.5, 114.1 (2C), 73.4, 65.5, 57.7, 55.2, 54.3, 49.7, 45.2, 43.5, 39.2, 38.7, 32.2, 25.7, 22.3, 21.5, $20.6 \mathrm{ppm}$;

HRMS (ESI): calculated for $\left[\mathrm{C}_{39} \mathrm{H}_{45} \mathrm{NO}_{6} \mathrm{~S}+\mathrm{H}\right]^{+}$656.3040, found 656.3033 .

\section{Compound ( \pm )-S1}<smiles>[Y5]N([Y5])C[C@@H]1CCC(=O)[C@@]2(CCOc3ccccc3)C=C(C(=C)C)C(=O)C[C@@]12C</smiles>

( \pm -19

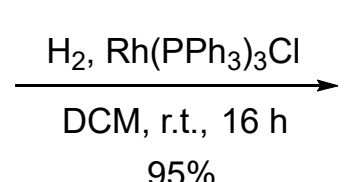

95\%

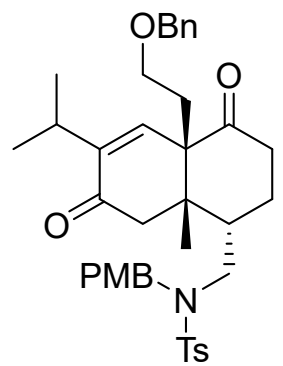

( \pm -S1

A solution of compound ( \pm )-19 (550 mg, $0.838 \mathrm{mmol}, 1.0$ equiv) and $\mathrm{Rh}\left(\mathrm{PPh}_{3}\right)_{3} \mathrm{Cl}$ (77.5 mg, $0.0838 \mathrm{mmol}, 0.10$ equiv) in dry DCM $(21 \mathrm{~mL})$ was stirred under an atmosphere of hydrogen for $16 \mathrm{~h}$. DCM was removed under reduced pressure and the residue was purified by flash chromatography on silica gel (eluent: EtOAc/PE, 1/10 to 1/5) to give compound $\mathbf{S 1}$ (524 $\mathrm{mg}$, yield: $95 \%$ ) as a white foam.

$\mathbf{R f}=0.45($ silica, $\mathrm{EtOAc} / \mathrm{PE}=1: 3)$

${ }^{1} \mathbf{H}$ NMR (400 MHz, $\left.\mathrm{CDCl}_{3}\right): \delta=7.69(\mathrm{~d}, J=8.2 \mathrm{~Hz}, 2 \mathrm{H}), 7.39-7.27(\mathrm{~m}, 7 \mathrm{H}), 7.22(\mathrm{~d}$, $J=8.6 \mathrm{~Hz}, 2 \mathrm{H}), 6.89-6.82(\mathrm{~m}, 2 \mathrm{H}), 6.39$ (d, $J=0.6 \mathrm{~Hz}, 1 \mathrm{H}), 4.45$ (d, $J=13.8 \mathrm{~Hz}$, 1H), 4.41 (d, $J=11.7 \mathrm{~Hz}, 1 \mathrm{H}), 4.37$ (d, $J=11.7 \mathrm{~Hz}, 1 \mathrm{H}), 3.88$ (d, $J=14.0 \mathrm{~Hz}, 1 \mathrm{H})$, $3.72(\mathrm{~s}, 3 \mathrm{H}), 3.52-3.44(\mathrm{~m}, 1 \mathrm{H}), 3.41-3.32(\mathrm{~m}, 1 \mathrm{H}), 3.03(\mathrm{dd}, J=13.6,11.6 \mathrm{~Hz}, 1 \mathrm{H})$, 2.89-2.73 (m, 2H), $2.45(\mathrm{~s}, 3 \mathrm{H}), 2.38-2.26(\mathrm{~m}, 1 \mathrm{H}), 2.23-2.05(\mathrm{~m}, 3 \mathrm{H}), 1.95-1.81(\mathrm{~m}$, $3 \mathrm{H}), 1.55(\mathrm{dt}, J=14.7,5.8 \mathrm{~Hz}, 1 \mathrm{H}), 1.24-1.15(\mathrm{~m}, 1 \mathrm{H}), 1.01(\mathrm{~d}, J=6.8 \mathrm{~Hz}, 3 \mathrm{H}), 0.98$ $(\mathrm{d}, J=6.8 \mathrm{~Hz}, 3 \mathrm{H}), 0.84(\mathrm{~s}, 3 \mathrm{H}) \mathrm{ppm}$;

${ }^{13}$ C NMR (101 MHz, $\left.\mathrm{CDCl}_{3}\right): \delta=212.6,197.3,159.7,143.7,143.7,141.5,137.7$, 
$135.4,130.2,129.9,128.4,128.1,127.7,127.7,127.2,114.0,73.4,65.6,57.2,55.2$, 54.3, 49.8, 45.0, 43.0, 39.1, 38.7, 32.3, 26.4, 25.7, 21.9, 21.5, 21.3, 20.5 ppm;

HRMS (ESI): calculated for $\left[\mathrm{C}_{39} \mathrm{H}_{47} \mathrm{NO}_{6} \mathrm{~S}+\mathrm{Na}\right]^{+}$680.3016, found 680.3009 .

\section{Compound ( \pm )-20}<smiles>[Y9]N([13CH3])C[C@@H]1CCC(=O)[C@@]2(CCOc3ccccc3)C=C(C(C)C)C(=O)C[C@@]12C</smiles>

$( \pm)-\mathrm{S} 1$

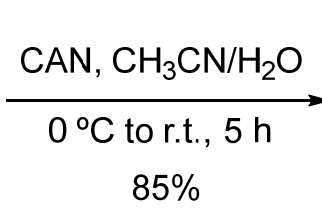

$85 \%$

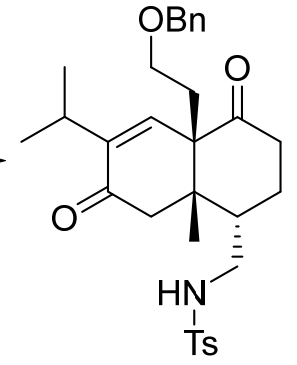

$( \pm)-20$

To a cooled $\left(0{ }^{\circ} \mathrm{C}\right)$ solution of enone $( \pm)-\mathbf{S 1}(658 \mathrm{mg}, 1.00 \mathrm{mmol}, 1.0$ equiv) in a mixture of $\mathrm{CH}_{3} \mathrm{CN}(28 \mathrm{~mL})$ and $\mathrm{H}_{2} \mathrm{O}(7 \mathrm{~mL})$, was added CAN $(2.20 \mathrm{~g}, 4.00 \mathrm{mmol}, 4.0$ equiv). After being stirred for $1 \mathrm{~h}$ at $0{ }^{\circ} \mathrm{C}$, the solution was warmed to room temperature and stirred for $4 \mathrm{~h}$. The crude mixture was partitioned between EtOAc $(60 \mathrm{~mL})$ and $\mathrm{H}_{2} \mathrm{O}(20 \mathrm{~mL})$, and the aqueous phase was extracted with EtOAc $(3 \times 30$ $\mathrm{mL})$. The combined organic phases were washed with $\mathrm{H}_{2} \mathrm{O}(3 \times 30 \mathrm{~mL})$, saturated aqueous $\mathrm{NaHCO}_{3}(30 \mathrm{~mL})$ and brine $(30 \mathrm{~mL})$, dried over anhydrous $\mathrm{Na}_{2} \mathrm{SO}_{4}$, filtered, and concentrated under reduced pressure. The residue was purified by flash chromatography on silica gel (eluent: EtOAc/PE, 1/4 to 1/2) to give compound ( \pm )-20 (459 mg, yield: $85 \%$ ) as a white foam.

$\mathbf{R f}=0.35$ (silica, EtOAc/PE $=1: 2$ );

${ }^{1} \mathbf{H}$ NMR (400 MHz, $\left.\mathrm{CDCl}_{3}\right): \delta=7.72(\mathrm{~d}, J=8.3 \mathrm{~Hz}, 2 \mathrm{H}), 7.38-7.25(\mathrm{~m}, 7 \mathrm{H}), 6.45(\mathrm{~d}$, $J=0.6 \mathrm{~Hz}, 1 \mathrm{H}), 5.23(\mathrm{t}, J=6.4 \mathrm{~Hz}, 1 \mathrm{H}), 4.42(\mathrm{~d}, J=11.5 \mathrm{~Hz}, 1 \mathrm{H}), 4.38(\mathrm{~d}, J=11.5$ $\mathrm{Hz}, 1 \mathrm{H}), 3.64-3.54(\mathrm{~m}, 1 \mathrm{H}), 3.54-3.44(\mathrm{~m}, 1 \mathrm{H}), 3.07$ (ddd, $J=12.7,5.5,2.4 \mathrm{~Hz}, 1 \mathrm{H})$, 2.88-2.76 (m, 1H), 2.76-2.63 (m, 1H), 2.52 (ddd, $J=13.1,9.9,7.1 \mathrm{~Hz}, 1 \mathrm{H}), 2.40$ (s, $3 \mathrm{H}), 2.38-2.10(\mathrm{~m}, 5 \mathrm{H}), 1.88(\mathrm{~d}, J=15.2 \mathrm{~Hz}, 1 \mathrm{H}), 1.70(\mathrm{dt}, J=14.5,5.4 \mathrm{~Hz}, 1 \mathrm{H})$, $1.47-1.35(\mathrm{~m}, 1 \mathrm{H}), 1.03$ (d, $J=6.8 \mathrm{~Hz}, 3 \mathrm{H}), 1.00(\mathrm{~s}, 3 \mathrm{H}), 0.99(\mathrm{~d}, J=6.8 \mathrm{~Hz}, 3 \mathrm{H})$ ppm;

${ }^{13} \mathbf{C}$ NMR (101 MHz, $\left.\mathrm{CDCl}_{3}\right): \delta=212.3,197.2,143.8,143.8,141.5,137.6,136.5$, 129.9 (2C), 128.4 (2C), 127.8 (3C), 127.0 (2C), 73.5, 65.6, 57.2, 45.1, 43.6, 43.1, 41.4, 
$38.5,32.5,26.4,26.2,21.9,21.4,21.3,20.8 \mathrm{ppm}$;

HRMS (ESI): calculated for $\left[\mathrm{C}_{31} \mathrm{H}_{39} \mathrm{NO}_{5} \mathrm{~S}+\mathrm{Na}\right]^{+}$560.2441, found 560.2437.

\section{Compound ( \pm )-22}<smiles>CC(C)C1=CC2(CCOCc3ccccc3)C(=O)CC[C@@H](CN[As])[C@]2(C)CC1=O</smiles>

$( \pm)-20$

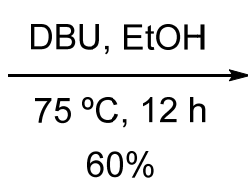

$60 \%$

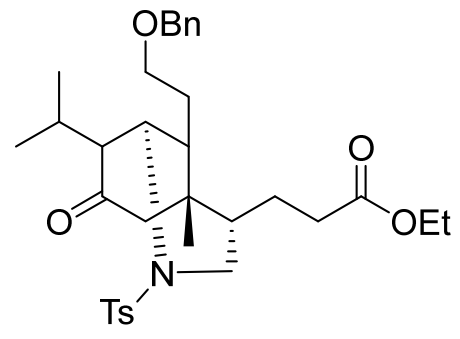

$( \pm)-22$

To a solution of compound ( \pm )-20 (53.8 mg, $0.100 \mathrm{mmol}, 1.0$ equiv) in EtOH (2 mL) under argon atmosphere, was added DBU (0.0299 mL, $0.200 \mathrm{mmol}, 2.0$ equiv). After being stirred for $12 \mathrm{~h}$ at $75{ }^{\circ} \mathrm{C}, 0.5 \mathrm{~N} \mathrm{HCl}(5 \mathrm{~mL})$ and EtOAc $(10 \mathrm{~mL})$ were added. The organic phase was separated and the aqueous phase was extracted with EtOAc (3 $\times 10 \mathrm{~mL}$ ). The combined organic phases were washed with brine, dried over anhydrous $\mathrm{Na}_{2} \mathrm{SO}_{4}$, filtered, and concentrated under reduced pressure. The residue was purified by flash chromatography on silica gel (eluent: EtOAc/PE, 1/3) to give compound ( \pm )-22 (35.0 mg, yield: $60 \%)$ as a colorless oil.

$\mathbf{R f}=0.55$ (silica, EtOAc/PE $=1: 2$ );

${ }^{1} \mathbf{H}$ NMR $\left(400 \mathrm{MHz}, \mathrm{CDCl}_{3}\right): \delta=7.63(\mathrm{~d}, J=8.3 \mathrm{~Hz}, 2 \mathrm{H}), 7.42-7.29(\mathrm{~m}, 5 \mathrm{H}), 7.09(\mathrm{~d}$, $J=8.0 \mathrm{~Hz}, 2 \mathrm{H}), 4.68(\mathrm{t}, J=3.4 \mathrm{~Hz}, 1 \mathrm{H}), 4.61(\mathrm{~d}, J=11.6 \mathrm{~Hz}, 1 \mathrm{H}), 4.57$ (d, $J=11.5$ Hz, 1H), 4.09 (q, $J=7.0 \mathrm{~Hz}, 2 \mathrm{H}), 3.66-3.58$ (m, 3H), 3.10-3.01 (m, 1H), 2.39-2.24 (m, 2H), $2.35(\mathrm{~s}, 3 \mathrm{H}), 2.22-2.16(\mathrm{~m}, 2 \mathrm{H}), 2.14-2.06(\mathrm{~m}, 1 \mathrm{H}), 1.99-1.82(\mathrm{~m}, 3 \mathrm{H})$, 1.74-1.52 (m, 3H), 1.24 (t, $J=7.0 \mathrm{~Hz}, 3 \mathrm{H}), 1.18-1.12(\mathrm{~m}, 1 \mathrm{H}), 1.00(\mathrm{~d}, J=6.6 \mathrm{~Hz}$, $3 \mathrm{H}), 0.94$ (s, 3H), $0.92(\mathrm{~d}, J=7.0 \mathrm{~Hz}, 3 \mathrm{H}) \mathrm{ppm}$;

${ }^{13}$ C NMR (101 MHz, $\left.\mathrm{CDCl}_{3}\right): \delta=209.7,172.9,143.1,138.6,137.8,129.4(2 \mathrm{C})$, 128.5 (2C), 128.1 (2C), 127.8, 126.8 (2C), 73.6, 68.3, 60.3, 57.4, 52.7, 52.3, 45.5, 41.0, 39.4, 38.8, 32.4, 27.0, 26.6, 25.4, 21.5, 21.4, 20.7, 19.5, 14.2 ppm;

HRMS (ESI): calculated for $\left[\mathrm{C}_{33} \mathrm{H}_{45} \mathrm{NO}_{6} \mathrm{~S}+\mathrm{Na}\right]^{+}$606.2860, found 606.2855. 


\section{Compound ( \pm )-23}<smiles>CC(C)C1=CC2(CCOCc3ccccc3)C(=O)CC[C@@H](CN[13F])[C@@]2(C)CC1=O</smiles>

$( \pm)-20$

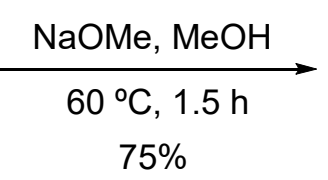

$75 \%$

To a solution of compound $( \pm)-\mathbf{2 0}(53.8 \mathrm{mg}, 0.100 \mathrm{mmol}, 1.0$ equiv) in $\mathrm{MeOH}(2 \mathrm{~mL})$ under argon atmosphere, was added $\mathrm{NaOMe}(27.0 \mathrm{mg}, 0.500 \mathrm{mmol}, 5.0$ equiv). After being stirred for $1.5 \mathrm{~h}$ at $60{ }^{\circ} \mathrm{C}, 0.5 \mathrm{~N} \mathrm{HCl}(6 \mathrm{~mL})$ and EtOAc $(15 \mathrm{~mL})$ were added. The organic phase was separated and the aqueous phase was extracted with EtOAc (3 $\times 10 \mathrm{~mL}$ ). The combined organic phases were washed with brine, dried over anhydrous $\mathrm{Na}_{2} \mathrm{SO}_{4}$, filtered, and concentrated under reduced pressure. The residue was purified by flash chromatography on silica gel (eluent: EtOAc/PE, 1/3) to give compound ( \pm )-23 (42.7 $\mathrm{mg}$, yield: 75\%) as a colorless oil.

$\mathbf{R f}=0.53($ silica, $\mathrm{EtOAc} / \mathrm{PE}=1: 2)$

${ }^{1} \mathbf{H}$ NMR $\left(400 \mathrm{MHz}, \mathrm{CDCl}_{3}\right): \delta=7.66-7.61(\mathrm{~m}, 2 \mathrm{H}), 7.43-7.28(\mathrm{~m}, 5 \mathrm{H}), 7.09(\mathrm{~d}, J=$ $8.0 \mathrm{~Hz}, 2 \mathrm{H}), 4.68(\mathrm{t}, J=3.1 \mathrm{~Hz}, 1 \mathrm{H}), 4.61(\mathrm{~d}, J=11.6 \mathrm{~Hz}, 1 \mathrm{H}), 4.57(\mathrm{~d}, J=11.5 \mathrm{~Hz}$, 1H), 3.69-3.56 (m, 3H), 3.64 (s, 3H), 3.05 (ddd, $J=16.1,3.6,1.9 \mathrm{~Hz}, 1 \mathrm{H}), 2.43-2.25$ (m, 2H), $2.36(\mathrm{~s}, 3 \mathrm{H}), 2.24-2.16(\mathrm{~m}, 2 \mathrm{H}), 2.16-2.04(\mathrm{~m}, 1 \mathrm{H}), 2.02-1.81(\mathrm{~m}, 3 \mathrm{H})$, 1.69-1.55 (m, 3H), 1.19-1.11 (m, 1H), 0.99 (d, $J=6.8 \mathrm{~Hz}, 3 \mathrm{H}), 0.94$ (s, 3H), 0.91 (d, $J=6.8 \mathrm{~Hz}, 3 \mathrm{H}) \mathrm{ppm}$;

${ }^{13} \mathrm{C}$ NMR (101 MHz, $\left.\mathrm{CDCl}_{3}\right): \delta=209.7,173.4,143.1,138.6,137.8,129.4(2 \mathrm{C})$, 128.5 (2C), 128.1 (2C), 127.8, 126.8 (2C), 73.6, 68.4, 57.4, 52.7, 52.4, 51.5, 45.6, 41.0, 39.5, 38.9, 32.2, 27.1, 26.7, 25.4, 21.6, 21.4, 20.7, 19.6 ppm;

HRMS (ESI): calculated for $\left[\mathrm{C}_{32} \mathrm{H}_{43} \mathrm{NO}_{6} \mathrm{~S}+\mathrm{Na}\right]^{+}$592.2703, found 592.2698. 


\section{Compound 4}
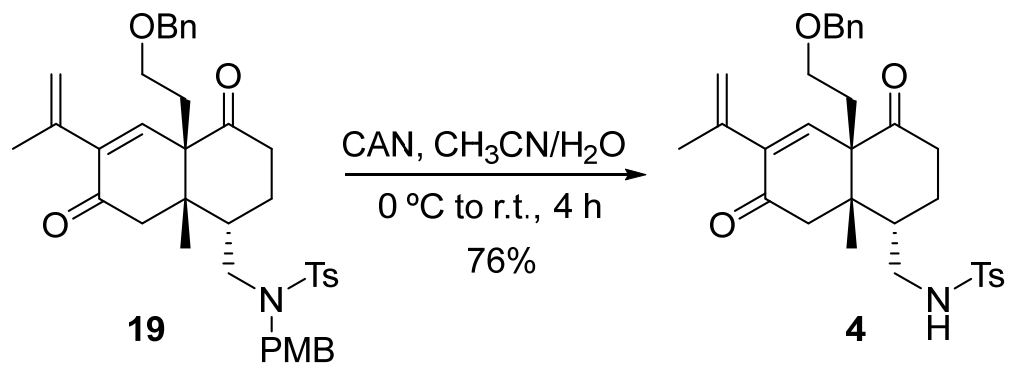

To a cooled $\left(0^{\circ} \mathrm{C}\right)$ solution of enone $19(6.56 \mathrm{~g}, 10.0 \mathrm{mmol}, 1.0$ equiv $)$ in a mixture of $\mathrm{CH}_{3} \mathrm{CN}(280 \mathrm{~mL})$ and $\mathrm{H}_{2} \mathrm{O}(70 \mathrm{~mL})$, was added CAN (22.0 g, $40.0 \mathrm{mmol}, 4.0$ equiv). After being stirred for $1 \mathrm{~h}$ at $0{ }^{\circ} \mathrm{C}$, the solution was warmed to room temperature and stirred for $4 \mathrm{~h}$. The crude mixture was partitioned between EtOAc $(500 \mathrm{~mL})$ and $\mathrm{H}_{2} \mathrm{O}$ $(200 \mathrm{~mL})$, and the aqueous phase was extracted with EtOAc $(3 \times 250 \mathrm{~mL})$. The combined organic phases were washed with $\mathrm{H}_{2} \mathrm{O}(3 \times 200 \mathrm{~mL})$, saturated aqueous $\mathrm{NaHCO}_{3}(200 \mathrm{~mL})$ and brine $(200 \mathrm{~mL})$, dried over anhydrous $\mathrm{Na}_{2} \mathrm{SO}_{4}$, filtered, and concentrated under reduced pressure. The residue was purified by flash chromatography on silica gel (eluent: EtOAc/PE, 1/4 to 1/2) to give compound 4 (4.08 g, yield: $76 \%)$ as a white foam.

$\mathbf{R f}=0.35($ silica, $\mathrm{EtOAc} / \mathrm{PE}=1: 2)$;

$[\alpha]_{\mathbf{D}^{20}}=+4.0\left(c 1.0, \mathrm{CHCl}_{3}\right) ;$

${ }^{1} \mathbf{H}$ NMR (400 MHz, $\left.\mathrm{CDCl}_{3}\right): \delta=7.75-7.69(\mathrm{~m}, 2 \mathrm{H}), 7.36-7.26(\mathrm{~m}, 7 \mathrm{H}), 6.68(\mathrm{~s}, 1 \mathrm{H})$, $5.17(\mathrm{dd}, J=2.2,1.0 \mathrm{~Hz}, 1 \mathrm{H}), 5.16-5.11(\mathrm{~m}, 1 \mathrm{H}), 5.07-5.03(\mathrm{~m}, 1 \mathrm{H}), 4.41(\mathrm{~d}, J=$ $11.5 \mathrm{~Hz}, 1 \mathrm{H}), 4.38(\mathrm{~d}, J=11.5 \mathrm{~Hz}, 1 \mathrm{H}), 3.60(\mathrm{dt}, J=10.8,5.5 \mathrm{~Hz}, 1 \mathrm{H}), 3.50$ (ddd, $J=$ 9.8, 8.0, $5.2 \mathrm{~Hz}, 1 \mathrm{H}), 3.12-3.04(\mathrm{~m}, 1 \mathrm{H}), 2.73(\mathrm{td}, J=14.2,6.3 \mathrm{~Hz}, 1 \mathrm{H}), 2.53(\mathrm{ddd}, J$ $=13.2,10.0,7.1 \mathrm{~Hz}, 1 \mathrm{H}), 2.41(\mathrm{~s}, 3 \mathrm{H}), 2.38-2.27(\mathrm{~m}, 3 \mathrm{H}), 2.26-2.13(\mathrm{~m}, 2 \mathrm{H})$, 1.93-1.87 (m, 4H), 1.48-1.35 (m, 1H), 1.04 (s, 3H) ppm;

${ }^{13}$ C NMR (101 MHz, $\left.\mathrm{CDCl}_{3}\right): \delta=212.1,196.7,144.9,143.8,140.2,139.8,137.5$, 136.5, 129.9 (2C), 128.4 (2C), 127.8 (3C), 127.0 (2C), 116.6, 73.5, 65.5, 57.6, 45.3, 43.7, 43.6, 41.5, 38.6, 32.4, 26.2, 22.3, 21.5, 20.9 ppm;

HRMS (ESI): calculated for $\left[\mathrm{C}_{31} \mathrm{H}_{37} \mathrm{NO}_{5} \mathrm{~S}+\mathrm{H}\right]^{+}$536.2465, found 536.2462. 


\section{Compound 24}
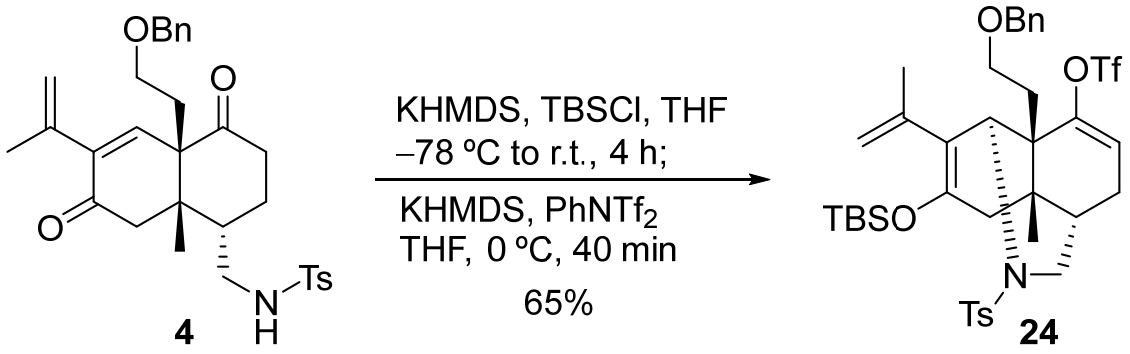

To a solution of enone 4 (1.07 g, $2.00 \mathrm{mmol}, 1.0$ equiv) and TBSCl (0.332 g, 2.20 mmol, 1.1 equiv) in THF $(20 \mathrm{ml})$ under argon atmosphere, was added a solution of 0.5 M KHMDS (4.20 mL, $2.10 \mathrm{mmol}, 1.05$ equiv) in toluene at $-78{ }^{\circ} \mathrm{C}$. After being stirred for $4 \mathrm{~h}$ at room temperature, the reaction mixture was recooled to $-78{ }^{\circ} \mathrm{C}$, then $\operatorname{PhNTf}_{2}(1.43 \mathrm{~g}, 4.00 \mathrm{mmol}, 2.0$ equiv) in THF (5 mL) and KHMDS (5.20 mL, 2.6 mmol, $0.5 \mathrm{M}, 1.3$ equiv) in toluene were added. The resulting mixture was warmed to $0{ }^{\circ} \mathrm{C}$ and stirred for $40 \mathrm{~min}$, then quenched with saturated aqueous $\mathrm{NH}_{4} \mathrm{Cl}(30 \mathrm{~mL})$. The organic phase was separated and the aqueous phase was extracted with EtOAc (3 $\times 30 \mathrm{~mL}$ ). The combined organic phases were washed with brine, dried over anhydrous $\mathrm{Na}_{2} \mathrm{SO}_{4}$, filtered, and concentrated under reduced pressure. The residue was purified by flash chromatography on silica gel (eluent: EtOAc/PE, 1/40 to 1/20) to give compound 24 (1.02 g, yield: 65\%) as a white solid.

$\mathbf{R f}=0.65($ silica $, \mathrm{EtOAc} / \mathrm{PE}=1: 9)$;

$[\alpha]_{\mathbf{D}^{20}}=+105.0\left(c 1.0, \mathrm{CHCl}_{3}\right)$

${ }^{1} \mathbf{H}$ NMR $\left(400 \mathrm{MHz}, \mathrm{CDCl}_{3}\right): \delta=7.62-7.57(\mathrm{~m}, 2 \mathrm{H}), 7.36-7.24(\mathrm{~m}, 5 \mathrm{H}), 7.18(\mathrm{~d}, J=$ $8.0 \mathrm{~Hz}, 2 \mathrm{H}), 5.67(\mathrm{dd}, J=5.0,2.8 \mathrm{~Hz}, 1 \mathrm{H}), 4.78(\mathrm{t}, J=1.6 \mathrm{~Hz}, 1 \mathrm{H}), 4.71(\mathrm{~s}, 1 \mathrm{H}), 4.59$ $(\mathrm{d}, J=11.6 \mathrm{~Hz}, 1 \mathrm{H}), 4.43(\mathrm{~d}, J=5.5 \mathrm{~Hz}, 1 \mathrm{H}), 4.41(\mathrm{~d}, J=5.5 \mathrm{~Hz}, 1 \mathrm{H}), 3.80(\mathrm{ddd}, J=$ 11.0, 8.2, $4.7 \mathrm{~Hz}, 1 \mathrm{H}), 3.68(\mathrm{ddd}, J=10.7,8.2,5.7 \mathrm{~Hz}, 1 \mathrm{H}), 3.47-3.35(\mathrm{~m}, 2 \mathrm{H})$, 2.44-2.32 (m, 1H), $2.39(\mathrm{~s}, 3 \mathrm{H}), 2.29-2.19(\mathrm{~m}, 1 \mathrm{H}), 2.10-1.96(\mathrm{~m}, 2 \mathrm{H}), 1.92(\mathrm{dd}, J=$ 18.6, $5.1 \mathrm{~Hz}, 1 \mathrm{H}), 1.83$ (ddd, $J=14.4,11.1,5.7 \mathrm{~Hz}, 1 \mathrm{H}), 1.51(\mathrm{~s}, 3 \mathrm{H}), 1.38-1.33$ (m, 1H), 1.02 (s, 3H), 0.88 (s, 9H), 0.09 (s, 3H), 0.06 (s, 3H) ppm;

${ }^{13} \mathrm{C}$ NMR (101 MHz, $\left.\mathrm{CDCl}_{3}\right): \delta=148.9,148.4,142.6,140.7,139.1,138.1,129.0$ (2C), 128.3 (2C), 128.0 (2C), 127.6, 126.9 (2C), 119.0, 117.3, 113.7, 73.4, 66.5, 57.0, $45.0,44.7,43.3,38.8,37.6,29.7,28.5,25.6$ (3C), 23.2, 22.2, 21.4, 17.9, -3.9, -4.0 
ppm;

HRMS (ESI): calculated for $\left[\mathrm{C}_{38} \mathrm{H}_{50} \mathrm{~F}_{3} \mathrm{NO}_{7} \mathrm{~S}_{2} \mathrm{Si}+\mathrm{H}\right]^{+}$782.2823, found 782.2814.

\section{Compound 25}

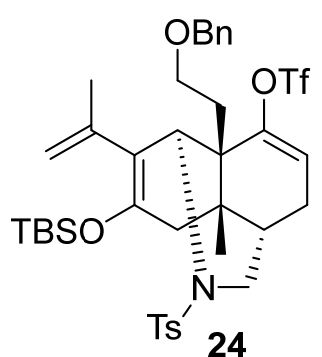

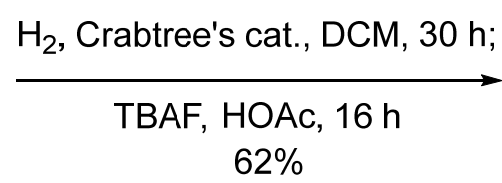

$62 \%$

A solution of compound 24 (1.17 g, $1.50 \mathrm{mmol}, 1.0$ equiv) and Crabtree's cat. (96.6 $\mathrm{mg}, 0.120 \mathrm{mmol}, 0.08$ equiv) in dry DCM (30 mL) was stirred under an atmosphere of hydrogen for $30 \mathrm{~h}$. DCM was removed under reduced pressure and the residue was dissolved in THF $(30 \mathrm{~mL})$. To the resulting mixture were added HOAc $(0.858 \mathrm{~mL}$, $15.0 \mathrm{mmol}, 10$ equiv) and TBAF (3.0 mL, $3.00 \mathrm{mmol}, 1.0 \mathrm{M}$ in THF, 2.0 equiv) at $0{ }^{\circ} \mathrm{C}$. The resulting mixture was warmed to room temperature and stirred for $16 \mathrm{~h}$, then quenched with saturated aqueous $\mathrm{NH}_{4} \mathrm{Cl}(30 \mathrm{~mL})$. The organic phase was separated and the aqueous phase was extracted with EtOAc $(4 \times 40 \mathrm{~mL})$. The combined organic phases were washed with brine $(2 \times 30 \mathrm{~mL})$, dried over anhydrous $\mathrm{Na}_{2} \mathrm{SO}_{4}$, filtered, and concentrated under reduced pressure. The residue was purified by flash chromatography on silica gel (eluent: EtOAc/PE, $1 / 10$ to $1 / 6$ ) to give compound $25(0.627 \mathrm{~g}$, yield: $62 \%)$ as a white foam.

$\mathbf{R f}=0.42($ silica, $\mathrm{EtOAc} / \mathrm{PE}=1: 3)$

$[\alpha]_{\mathbf{D}}^{20}=+41.5\left(c 2.0, \mathrm{CHCl}_{3}\right)$;

${ }^{1}$ H NMR (400 MHz, $\left.\mathrm{CDCl}_{3}\right): \delta=7.61-7.56(\mathrm{~m}, 2 \mathrm{H}), 7.36-7.26(\mathrm{~m}, 5 \mathrm{H}), 7.23(\mathrm{~d}, J=$ $8.2 \mathrm{~Hz}, 2 \mathrm{H}), 5.74(\mathrm{dd}, J=5.2,2.7 \mathrm{~Hz}, 1 \mathrm{H}), 4.57$ (d, $J=3.1 \mathrm{~Hz}, 1 \mathrm{H}), 4.56$ (d, $J=11.5$ $\mathrm{Hz}, 1 \mathrm{H}), 4.48$ (d, $J=11.5 \mathrm{~Hz}, 1 \mathrm{H}), 3.94(\mathrm{dt}, J=9.6,6.9 \mathrm{~Hz}, 1 \mathrm{H}), 3.76$ (ddd, $J=9.7$, 7.7, 5.1 Hz, 1H), 3.69 (d, $J=15.5 \mathrm{~Hz}, 1 \mathrm{H}), 3.30$ (ddd, $J=15.5,3.7,1.6 \mathrm{~Hz}, 1 \mathrm{H}), 2.65$ (d, $J=16.9 \mathrm{~Hz}, 1 \mathrm{H}), 2.54-2.41(\mathrm{~m}, 2 \mathrm{H}), 2.40$ (s, 3H), 2.36-2.27 (m, 2H), 2.20-2.05 (m, 2H), 1.57 (dt, $J=13.6,6.7 \mathrm{~Hz}, 1 \mathrm{H}), 1.47$ (t, $J=4.1 \mathrm{~Hz}, 1 \mathrm{H}), 1.03$ (s, 3H), 0.88 (d, $J=6.4 \mathrm{~Hz}, 3 \mathrm{H}), 0.78(\mathrm{~d}, J=6.7 \mathrm{~Hz}, 3 \mathrm{H}) \mathrm{ppm}$; 
${ }^{13}$ C NMR (101 MHz, $\left.\mathrm{CDCl}_{3}\right): \delta=209.1,148.3,143.1,139.0,137.6,129.4$ (2C), 128.5 (2C), 128.1 (2C), 127.9, 126.3 (2C), 119.1, 118.2 (q, $J=320 \mathrm{~Hz}), 73.5,66.1$, 58.4, 55.1, 52.1, 46.5, 46.0, 41.5, 38.1, 30.2, 28.5, 26.4, 22.6, 21.4, 21.2, 20.8 ppm;

HRMS (ESI): calculated for $\left[\mathrm{C}_{32} \mathrm{H}_{38} \mathrm{~F}_{3} \mathrm{NO}_{7} \mathrm{~S}_{2}+\mathrm{Na}\right]^{+}$692.1934, found 692.1930 .

\section{Compound S2}
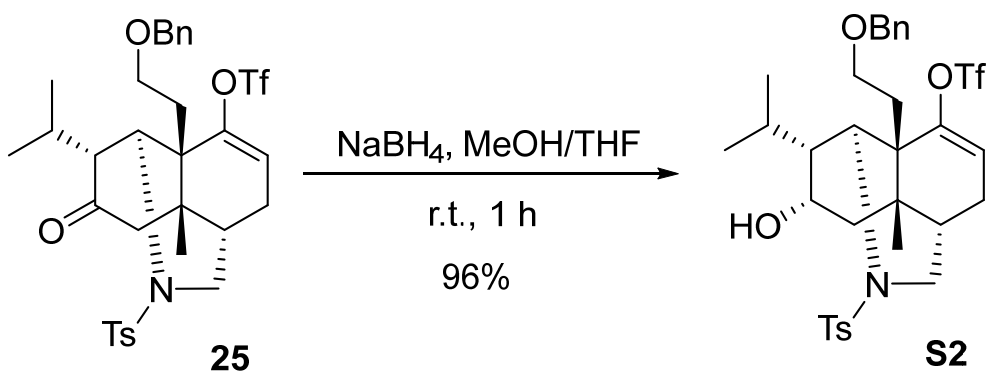

To a solution of compound 25 ( $1.34 \mathrm{~g}, 2.00 \mathrm{mmol}, 1.0$ equiv) in a mixture of $\mathrm{MeOH}$ $(20 \mathrm{~mL})$ and THF $(20 \mathrm{~mL})$ was added $\mathrm{NaBH}_{4}(760 \mathrm{mg}, 20.0 \mathrm{mmol}, 10$ equiv) over 15 min. The resulting mixture was stirred at room temperature for $1 \mathrm{~h}$, then quenched with $2 N \mathrm{HCl}(20 \mathrm{~mL})$. The organic phase was separated and the aqueous phase was extracted with DCM $(4 \times 40 \mathrm{~mL})$. The combined organic phases were washed with brine, dried over anhydrous $\mathrm{Na}_{2} \mathrm{SO}_{4}$, filtered, and concentrated under reduced pressure. The residue was purified by flash chromatography on silica gel (eluent: EtOAc/PE, 1/2) to give compound S2 (1.29 g, yield: 96\%) as a white foam.

$\mathbf{R f}=0.35($ silica $, \mathrm{EtOAc} / \mathrm{PE}=1: 3)$

$[\alpha]_{\mathbf{D}}^{\mathbf{2 0}}=+29.0\left(c 1.0, \mathrm{CHCl}_{3}\right)$;

${ }^{1} \mathbf{H}$ NMR (400 MHz, $\left.\mathrm{CDCl}_{3}\right): \delta=7.66-7.62(\mathrm{~m}, 2 \mathrm{H}), 7.36-7.27(\mathrm{~m}, 5 \mathrm{H}), 7.22(\mathrm{~d}, J=$ $8.1 \mathrm{~Hz}, 2 \mathrm{H}), 5.64(\mathrm{dd}, J=5.5,2.5 \mathrm{~Hz}, 1 \mathrm{H}), 4.89-4.78(\mathrm{~m}, 1 \mathrm{H}), 4.54(\mathrm{~d}, J=11.5 \mathrm{~Hz}$, $1 \mathrm{H}), 4.45(\mathrm{~d}, J=11.5 \mathrm{~Hz}, 1 \mathrm{H}), 4.26(\mathrm{~d}, J=2.4 \mathrm{~Hz}, 1 \mathrm{H}), 4.17-4.09(\mathrm{~m}, 1 \mathrm{H}), 3.86(\mathrm{td}$, $J=9.1,6.4 \mathrm{~Hz}, 1 \mathrm{H}), 3.60(\mathrm{td}, J=9.4,4.1 \mathrm{~Hz}, 1 \mathrm{H}), 3.36(\mathrm{~d}, J=13.7 \mathrm{~Hz}, 1 \mathrm{H})$, 2.44-2.34 (m, 1H), 2.40 (s, 3H), 2.21 (dd, $J=16.5,7.1 \mathrm{~Hz}, 1 \mathrm{H}), 2.07$ (ddd, $J=15.5$, 9.5, 6.4 Hz, 1H), $1.88(\mathrm{dd}, J=18.3,5.5 \mathrm{~Hz}, 1 \mathrm{H}), 1.80(\mathrm{ddd}, J=15.1,8.9,4.2 \mathrm{~Hz}, 1 \mathrm{H})$, $1.71(\mathrm{~d}, J=2.8 \mathrm{~Hz}, 1 \mathrm{H}), 1.66-1.61(\mathrm{~m}, 1 \mathrm{H}), 1.55-1.38(\mathrm{~m}, 2 \mathrm{H}), 1.35(\mathrm{t}, J=5.2 \mathrm{~Hz}$, $1 \mathrm{H}), 0.94(\mathrm{~d}, J=6.0 \mathrm{~Hz}, 3 \mathrm{H}), 0.92(\mathrm{~d}, J=5.7 \mathrm{~Hz}, 3 \mathrm{H}), 0.91$ (s, 3H) ppm;

${ }^{13}$ C NMR (101 MHz, $\left.\mathrm{CDCl}_{3}\right): \delta=149.9,142.6,138.7,137.9,129.1$ (2C), $128.5(2 \mathrm{C})$, 
128.0 (2C), 127.8, 126.9 (2C), 117.8, 73.3, 66.2, 63.1, 52.4, 47.2, 45.8, 44.6, 42.9, $36.8,35.4,30.1,30.0,25.7,24.3,21.6,21.5,21.1 \mathrm{ppm}$;

HRMS (ESI): calculated for $\left[\mathrm{C}_{32} \mathrm{H}_{40} \mathrm{~F}_{3} \mathrm{NO}_{7} \mathrm{~S}_{2}+\mathrm{Na}\right]^{+}$694.2090, found 694.2084.

\section{Compound 26}
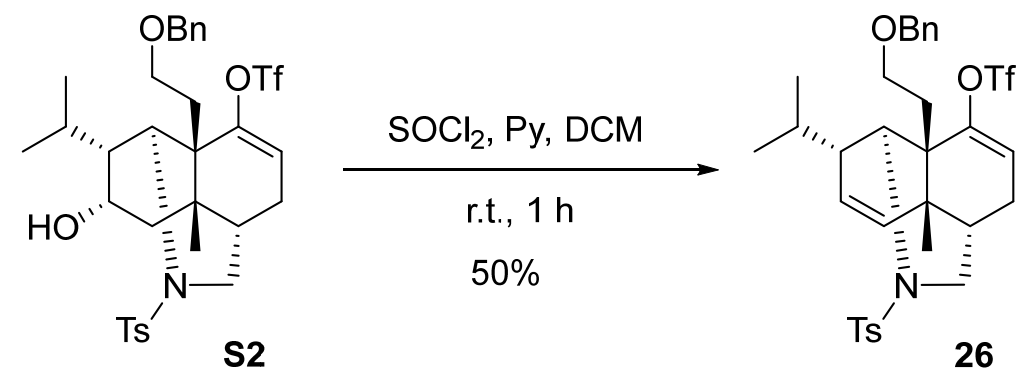

To a solution of compound $\mathbf{S 2}(672 \mathrm{mg}, 1.00 \mathrm{mmol}, 1.0$ equiv) in a mixture of pyridine $(5 \mathrm{~mL})$ and DCM $(5 \mathrm{~mL})$ under argon atmosphere, was added $\mathrm{SOCl}_{2}(0.726$ $\mathrm{mL}, 10.0 \mathrm{mmol}, 10$ equiv) dropwise at room temperature. The resulting mixture was stirred at this temperature for $1 \mathrm{~h}$, then quenched with $15 \% \mathrm{NaOH}$ aq. $(15 \mathrm{~mL})$ at $0{ }^{\circ} \mathrm{C}$. The mixture was partitioned between EtOAc $(20 \mathrm{~mL})$ and $\mathrm{H}_{2} \mathrm{O}(10 \mathrm{~mL})$, and the aqueous phase was extracted with EtOAc $(3 \times 20 \mathrm{~mL})$. The combined organic phases were washed with brine $(200 \mathrm{~mL})$, dried over anhydrous $\mathrm{Na}_{2} \mathrm{SO}_{4}$, filtered, and concentrated under reduced pressure. The residue was purified by flash chromatography on silica gel (eluent: EtOAc/PE, 1/40 to 1/20) to give compound 26 (330 mg, yield: 50\%) as a white foam.

$\mathbf{R f}=0.65($ silica, $\mathrm{EtOAc} / \mathrm{PE}=1: 5)$;

$[\alpha]_{\mathbf{D}}{ }^{\mathbf{2 0}}=+138.0\left(c 1.0, \mathrm{CHCl}_{3}\right)$;

${ }^{1} \mathbf{H}$ NMR (400 MHz, $\mathrm{CDCl}_{3}$ ): $\delta=7.67-7.62(\mathrm{~m}, 2 \mathrm{H}), 7.37-7.20(\mathrm{~m}, 7 \mathrm{H}), 5.95$ (ddd, $J$ $=10.4,2.3,1.2 \mathrm{~Hz}, 1 \mathrm{H}), 5.51(\mathrm{dd}, J=5.2,2.7 \mathrm{~Hz}, 1 \mathrm{H}), 5.21(\mathrm{dd}, J=10.4,3.0 \mathrm{~Hz}$, $1 \mathrm{H}), 4.53(\mathrm{~d}, J=11.6 \mathrm{~Hz}, 1 \mathrm{H}), 4.45(\mathrm{~d}, J=11.6 \mathrm{~Hz}, 1 \mathrm{H}), 4.23(\mathrm{~d}, J=4.3 \mathrm{~Hz}, 1 \mathrm{H})$, 3.92-3.83 (m, 1H), $3.64(\mathrm{td}, J=9.2,4.5 \mathrm{~Hz}, 1 \mathrm{H}), 3.53(\mathrm{~d}, J=2.5 \mathrm{~Hz}, 2 \mathrm{H}), 2.41(\mathrm{~s}$, 3H), $2.36(\mathrm{ddd}, J=18.7,6.0,2.7 \mathrm{~Hz}, 1 \mathrm{H}), 2.19$ (ddt, $J=7.4,5.3,2.7 \mathrm{~Hz}, 1 \mathrm{H}$ ), 2.14-1.89 (m, 3H), $1.52(\mathrm{dt}, J=13.5,6.7 \mathrm{~Hz}, 1 \mathrm{H}), 1.30-1.23(\mathrm{~m}, 1 \mathrm{H}), 1.02(\mathrm{~s}, 3 \mathrm{H})$, $0.87(\mathrm{~d}, J=6.7 \mathrm{~Hz}, 3 \mathrm{H}), 0.82(\mathrm{~d}, J=6.5 \mathrm{~Hz}, 3 \mathrm{H}) \mathrm{ppm}$;

${ }^{13} \mathrm{C}$ NMR (101 MHz, $\left.\mathrm{CDCl}_{3}\right): \delta=149.5,142.8,139.0,138.1,131.4,129.2$ (3C), 
128.4 (2C), 127.9 (2C), 127.6, 126.9 (2C), 118.3, 73.1, 66.9, 55.0, 47.6, 45.5, 43.2, 38.8, 34.8, 30.4, 28.0, 26.8, 23.1, 21.4, 21.1, $20.1 \mathrm{ppm}$;

HRMS (ESI): calculated for $\left[\mathrm{C}_{32} \mathrm{H}_{38} \mathrm{~F}_{3} \mathrm{NO}_{6} \mathrm{~S}_{2}+\mathrm{Na}\right]^{+}$676.1985, found 676.1977.

\section{Compound 27}<smiles>Cc1cccc(C)c1N</smiles>

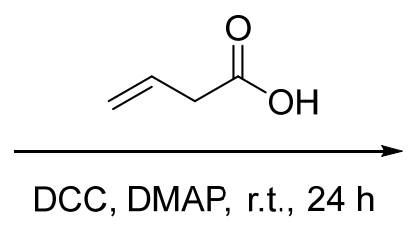

$52 \%$<smiles>C=CCC(=O)Nc1c(C)cccc1C</smiles>

27

To a cooled $\left(0{ }^{\circ} \mathrm{C}\right)$ solution of DCC $(12.4 \mathrm{~g}, 60.1 \mathrm{mmol}, 1.0$ equiv) and DMAP (733 mg, $6.00 \mathrm{mmol}, 0.1$ equiv) in DCM (200 mL) under argon atmosphere, were added Vinyl acetic caid (6.12 mL, $72.0 \mathrm{mmol}, 1.2$ equiv) and 2,6-dimethylaniline (8.86 mL, $72.0 \mathrm{mmol}, 1.2 \mathrm{eq})$. After being stirred for $24 \mathrm{~h}$ at room temperature, the reaction mixture was filtered through a plug of $\mathrm{SiO}_{2}$ topped with Celite (eluent: DCM). The filtrate was concentrated under reduced pressure. The residue was purified by flash chromatography on silica gel (eluent: EtOAc/PE, 1/6) to give compound 27 (5.92 g, yield: $52 \%$ ) as a white solid.

$\mathbf{R f}=0.40($ silica, $\mathrm{EtOAc} / \mathrm{PE}=1: 2)$

${ }^{1} \mathbf{H}$ NMR (400 MHz, $\mathrm{CDCl}_{3}$, data of two rotamers, ratio = 8:1): $\delta=7.22-6.95(\mathrm{~m}, 4 \mathrm{H})$, 6.07 (ddt, $J=17.2,10.1,7.2 \mathrm{~Hz}, 0.89 \mathrm{H}), 5.89$ (ddt, $J=17.2,10.1,7.1 \mathrm{~Hz}, 0.11 \mathrm{H}$ ), 5.37-5.29 (m, 1.78H), 5.09 (ddd, $J=10.1,2.8,1.5 \mathrm{~Hz}, 0.11 \mathrm{H}), 4.93$ (ddd, $J=17.1$, 3.0, $1.5 \mathrm{~Hz}, 0.11 \mathrm{H}), 3.19(\mathrm{dt}, J=7.3,1.1 \mathrm{~Hz}, 1.78 \mathrm{H}), 2.73(\mathrm{dt}, J=6.9,1.4 \mathrm{~Hz}, 0.22 \mathrm{H})$, $2.26(\mathrm{~s}, 0.67 \mathrm{H}), 2.19(\mathrm{~s}, 5.33 \mathrm{H}) \mathrm{ppm}$;

${ }^{13} \mathbf{C ~ N M R}\left(101 \mathrm{MHz}, \mathrm{CDCl}_{3}\right.$, two rotamers, ratio = 8:1, data of the major rotamer was given): $\delta=168.9,135.3$ (2C), 133.7, 131.5, 128.1 (2C), 127.3, 120.1, 41.7, 18.3 (2C) ppm;

HRMS (ESI): calculated for $\left[\mathrm{C}_{12} \mathrm{H}_{15} \mathrm{NO}+\mathrm{H}\right]^{+}$190.1226, found 190.1226 . 


\section{Compound 3}

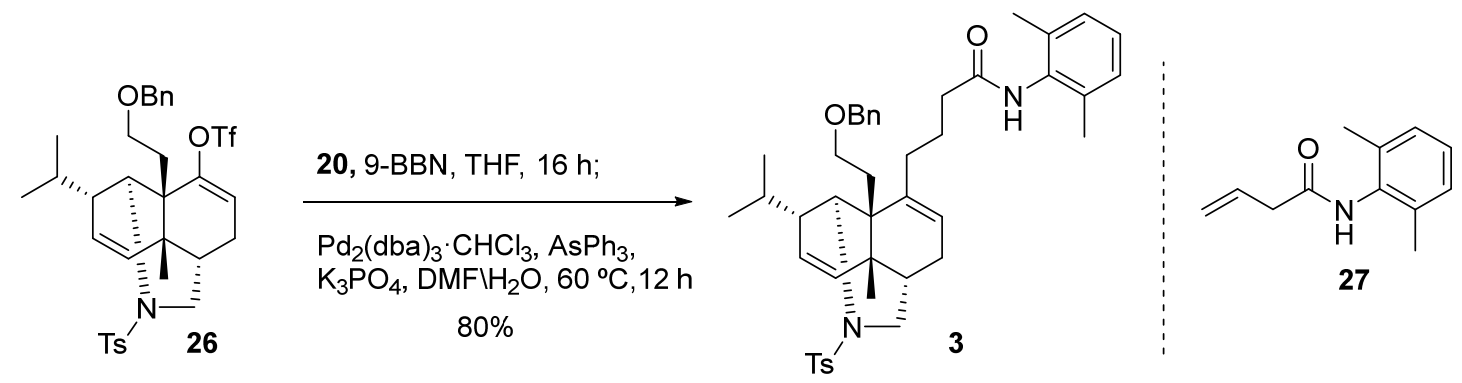

A solution of compound 27 (284 mg, $1.50 \mathrm{mmol}, 3.0$ equiv) and 9-BBN (6.0 mL, 3.0 mmol, $0.5 \mathrm{M}$ in THF, 6 equiv) was stirred at room temperature for $16 \mathrm{~h}$. An aqueous solution of $\mathrm{K}_{3} \mathrm{PO}_{4}(2.00 \mathrm{~mL}, 6.00 \mathrm{mmol}, 3.0 \mathrm{M}, 12$ equiv) was added slowly and the resulting biphasic mixture was stirred vigorously for $3 \mathrm{~h}$. A solution of enol triflate $\mathbf{2 6}$ (327 mg, $0.500 \mathrm{mmol}, 1.0$ equiv) in DMF (12 mL) was added and the mixture was purged from traces of hydrogen gas (vacuum was applied until the mixture started to boil and then the flask was refilled with argon). AsPh $(61.2 \mathrm{mg}, 0.200 \mathrm{mmol}, 0.4$ equiv) and $\mathrm{Pd}_{2}(\mathrm{dba})_{3} \bullet \mathrm{CHCl}_{3}(51.8 \mathrm{mg}, 0.0500 \mathrm{mmol}, 0.1$ equiv) were added and the resulting mixture was warmed to $60{ }^{\circ} \mathrm{C}$. The resulting mixture was stirred at this temperature for $12 \mathrm{~h}$. The reaction mixture was cooled to room temperature, diluted with EtOAc $(100 \mathrm{ml})$ and poured into water $(50 \mathrm{ml})$. The organic phase was separated and the aqueous phase was extracted with EtOAc $(3 \times 50 \mathrm{~mL})$. The combined organic phases were washed with brine, dried over anhydrous $\mathrm{Na}_{2} \mathrm{SO}_{4}$, filtered, and concentrated under reduced pressure. The residue was purified by flash chromatography on silica gel (eluent: EtOAc/PE, 1/10 to 1/3) to give compound 3 (279 mg, yield: 80\%) as a white foam.

$\mathbf{R f}=0.25($ silica $, \mathrm{EtOAc} / \mathrm{PE}=1: 3)$

$[\alpha]_{\mathbf{D}}{ }^{20}=+71.0\left(c 1.0, \mathrm{CHCl}_{3}\right)$

${ }^{1} \mathbf{H}$ NMR $\left(400 \mathrm{MHz}, \mathrm{CDCl}_{3}\right.$, data of two rotamers, ratio $\left.=10: 1\right): \delta=7.57(\mathrm{~d}, J=8.2$ $\mathrm{Hz}, 0.18 \mathrm{H}), 7.46(\mathrm{~d}, J=8.2 \mathrm{~Hz}, 1.82 \mathrm{H}), 7.41-7.23(\mathrm{~m}, 6 \mathrm{H}), 7.19(\mathrm{~d}, J=8.1 \mathrm{~Hz}$, $0.18 \mathrm{H}), 7.46(\mathrm{~d}, J=8.1 \mathrm{~Hz}, 1.82 \mathrm{H}), 7.13-7.03(\mathrm{~m}, 3 \mathrm{H}), 5.84(\mathrm{~d}, J=10.2 \mathrm{~Hz}, 1 \mathrm{H})$, $5.62(\mathrm{~s}, 0.91 \mathrm{H}), 5.35(\mathrm{~s}, 0.09 \mathrm{H}), 5.23(\mathrm{dd}, J=10.4,2.9 \mathrm{~Hz}, 1 \mathrm{H}), 4.49(\mathrm{~d}, J=11.8 \mathrm{~Hz}$, $0.91 \mathrm{H}), 4.42(\mathrm{~d}, J=11.8 \mathrm{~Hz}, 0.91 \mathrm{H}), 4.38(\mathrm{~s}, 0.18 \mathrm{H}), 4.00(\mathrm{~d}, J=4.4 \mathrm{~Hz}, 0.91 \mathrm{H})$, $3.91(\mathrm{~d}, J=4.4 \mathrm{~Hz}, 0.09 \mathrm{H}), 3.69-3.32(\mathrm{~m}, 4 \mathrm{H}), 2.64-2.43(\mathrm{~m}, 2 \mathrm{H}), 2.43-2.32(\mathrm{~m}, 4 \mathrm{H})$, 
$2.24(\mathrm{~s}, 6 \mathrm{H}), 2.21-1.90(\mathrm{~m}, 7 \mathrm{H}), 1.82-1.71(\mathrm{~m}, 1 \mathrm{H}), 1.27-1.20(\mathrm{~m}, 1 \mathrm{H}), 1.18-1.06(\mathrm{~m}$, $1 \mathrm{H}), 0.99(\mathrm{~s}, 2.73 \mathrm{H}), 0.90(\mathrm{~s}, 0.27 \mathrm{H}), 0.81-0.77(\mathrm{~m}, 0.54 \mathrm{H}), 0.74(\mathrm{~d}, J=6.6 \mathrm{~Hz}$, $2.73 \mathrm{H}), 0.69(\mathrm{~d}, J=6.4 \mathrm{~Hz}, 2.73 \mathrm{H}) \mathrm{ppm}$;

${ }^{13} \mathbf{C ~ N M R}\left(101 \mathrm{MHz}, \mathrm{CDCl}_{3}\right.$, two rotamers, ratio = 10:1, data of the major rotamer was given): $\delta=171.8,142.2,139.5,138.2,135.9,135.6,134.3,134.1,129.0(2 \mathrm{C})$, 128.4 (2C), 128.0 (2C), 127.7 (2C), 127.6 (2C), 127.5, 127.0, 126.3 (2C), 124.1, 73.0, 68.6, 56.7, 47.9, 45.6, 43.7, 37.1, 36.4, 35.7, 32.6, 30.2, 28.0 (2C), 23.0 (2C), 22.7, 21.4, 20.4, 18.5 (2C) ppm;

HRMS (ESI): calculated for $\left[\mathrm{C}_{43} \mathrm{H}_{54} \mathrm{~N}_{2} \mathrm{O}_{4} \mathrm{~S}+\mathrm{H}\right]^{+}$695.3877, found 695.3870 .

\section{Compound 28 and 34}
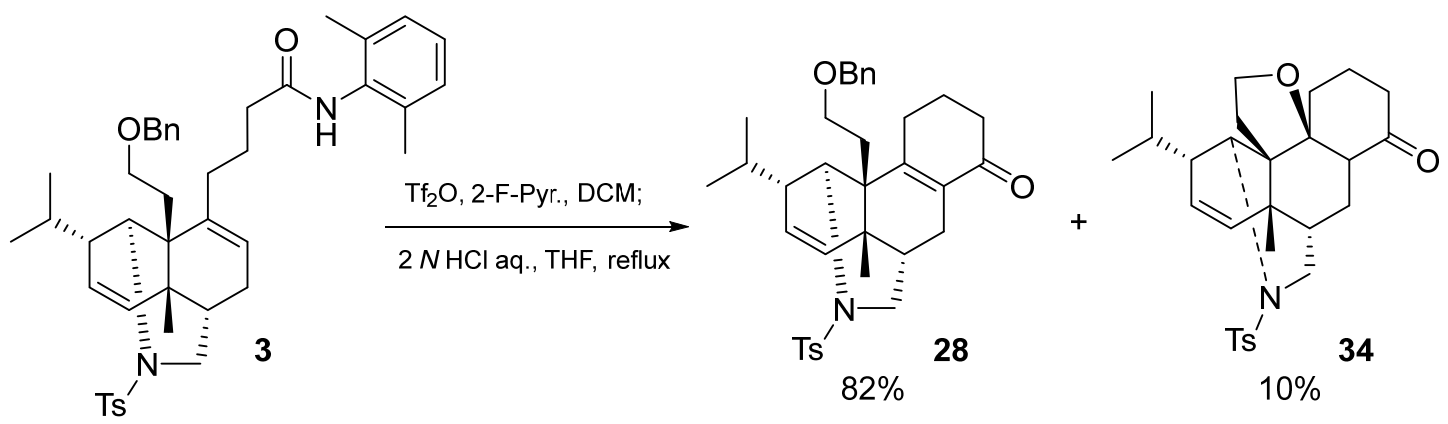

To a cooled $\left(0^{\circ} \mathrm{C}\right)$ solution of compound $3(695 \mathrm{mg}, 1.00 \mathrm{mmol}, 1.0$ equiv) and 2-Fluoropyridine $(0.155 \mathrm{~mL}, 1.80 \mathrm{mmol}, 1.8$ equiv) in $\mathrm{DCM}(15 \mathrm{~mL})$ under argon atmosphere, was added $\mathrm{Tf}_{2} \mathrm{O}(0.220 \mathrm{~mL} 1.30 \mathrm{mmol}, 1.3$ equiv $)$. After being stirred for $15 \mathrm{~min}$ at $0{ }^{\circ} \mathrm{C}$, the solution was warmed to room temperature and stirred for $1 \mathrm{~h}$. DCM was removed under reduced pressure and the residue was dissolved in THF (20 $\mathrm{mL})$ and $2 \mathrm{~N} \mathrm{HCl}(10 \mathrm{~mL})$. The resulting mixture was warmed to refluxing and stirred for $16 \mathrm{~h}$. The reaction mixture was cooled to room temperature, diluted with EtOAc $(20 \mathrm{ml})$. The organic phase was separated and the aqueous phase was extracted with EtOAc $(3 \times 15 \mathrm{~mL})$. The combined organic phases were washed with brine, dried over anhydrous $\mathrm{Na}_{2} \mathrm{SO}_{4}$, filtered, and concentrated under reduced pressure. The residue was purified by flash chromatography on silica gel (eluent: EtOAc/PE, 1/8 to 1/4) to give, in order of elution, compound 34 (47 mg, yield: 10\%) and compound 28 (473 mg, yield: 82\%) each as a pale-yellow oil. 
Data for compound 34:

$\mathbf{R f}=0.42($ silica, $\mathrm{EtOAc} / \mathrm{PE}=1: 4)$

$[\alpha]_{\mathbf{D}}^{\mathbf{2 0}}=+107\left(c 1.0, \mathrm{CHCl}_{3}\right)$

${ }^{1} \mathbf{H}$ NMR $\left(400 \mathrm{MHz}, \mathrm{CDCl}_{3}\right): \delta=7.67(\mathrm{~d}, J=8.3 \mathrm{~Hz}, 2 \mathrm{H}), 7.25(\mathrm{~d}, J=8.1 \mathrm{~Hz}, 2 \mathrm{H})$, $5.77(\mathrm{dt}, J=10.3,1.4 \mathrm{~Hz}, 1 \mathrm{H}), 5.08(\mathrm{dd}, J=10.3,3.1 \mathrm{~Hz}, 1 \mathrm{H}), 4.34(\mathrm{~d}, J=3.0 \mathrm{~Hz}$, $1 \mathrm{H}), 3.71(\mathrm{~d}, J=12.3 \mathrm{~Hz}, 1 \mathrm{H}), 3.66(\mathrm{~d}, J=8.1 \mathrm{~Hz}, 1 \mathrm{H}), 3.64(\mathrm{~d}, J=8.1 \mathrm{~Hz}, 1 \mathrm{H})$, 3.48-3.45 (m, 1H), 3.45-3.41 (m, 1H), $2.40(\mathrm{~s}, 3 \mathrm{H}), 2.38-2.22(\mathrm{~m}, 4 \mathrm{H}), 2.03-1.93(\mathrm{~m}$, 2H), 1.91-1.77 (m, 3H), 1.76-1.68 (m, 1H), 1.61-1.50 (m, 2H), 1.27-1.18 (m, 1H), $1.23(\mathrm{~s}, 3 \mathrm{H}), 1.03(\mathrm{~d}, J=6.4 \mathrm{~Hz}, 3 \mathrm{H}), 0.67$ (d, $J=6.6 \mathrm{~Hz}, 3 \mathrm{H}) \mathrm{ppm}$;

${ }^{13} \mathrm{C}$ NMR (101 MHz, $\left.\mathrm{CDCl}_{3}\right): \delta=210.8,143.0,138.2,135.5,129.3$ (2C), 127.2, 126.8 (2C), 87.8, 62.2, 56.9, 49.6, 49.2, 47.4, 46.6, 40.2, 37.2, 36.2, 32.0, 28.7, 27.3, 23.7, 23.5, 21.6, 21.4, 20.4, $20.2 \mathrm{ppm}$;

HRMS (ESI): calculated for $\left[\mathrm{C}_{28} \mathrm{H}_{37} \mathrm{NO}_{4} \mathrm{~S}+\mathrm{H}\right]^{+} 484.2516$, found 484.2511 .

Data for compound 28:

$\mathbf{R f}=0.35($ silica, $\mathrm{EtOAc} / \mathrm{PE}=1: 4)$

$[\alpha]_{\mathbf{D}}{ }^{20}=+88.0\left(c 0.5, \mathrm{CHCl}_{3}\right) ;$

${ }^{1} \mathbf{H}$ NMR $\left(400 \mathrm{MHz}, \mathrm{CDCl}_{3}\right): \delta=7.58(\mathrm{~d}, J=8.2 \mathrm{~Hz}, 2 \mathrm{H}), 7.39-7.26(\mathrm{~m}, 5 \mathrm{H}), 7.19(\mathrm{~d}$, $J=8.1 \mathrm{~Hz}, 2 \mathrm{H}), 5.84(\mathrm{~d}, J=10.3 \mathrm{~Hz}, 1 \mathrm{H}), 5.24(\mathrm{dd}, J=10.4,3.0 \mathrm{~Hz}, 1 \mathrm{H}), 4.52(\mathrm{~d}, J$ $=11.9 \mathrm{~Hz}, 1 \mathrm{H}), 4.40(\mathrm{~d}, J=11.9 \mathrm{~Hz}, 1 \mathrm{H}), 4.15(\mathrm{~d}, J=4.6 \mathrm{~Hz}, 1 \mathrm{H}), 3.58(\mathrm{dt}, J=9.0$, $7.4 \mathrm{~Hz}, 1 \mathrm{H}), 3.50(\mathrm{~d}, J=13.4 \mathrm{~Hz}, 1 \mathrm{H}), 3.40(\mathrm{td}, J=8.8,3.2 \mathrm{~Hz}, 1 \mathrm{H}), 3.26(\mathrm{dd}, J=$ 13.3, $2.8 \mathrm{~Hz}, 1 \mathrm{H}), 2.61(\mathrm{~d}, J=18.6 \mathrm{~Hz}, 1 \mathrm{H}), 2.57-2.41(\mathrm{~m}, 3 \mathrm{H}), 2.37(\mathrm{~s}, 3 \mathrm{H})$, 2.34-2.10 (m, 4H), 1.99-1.92 (m, 1H), 1.90-1.72 (m, 2H), 1.41-1.36 (m, 1H), $1.19-1.08(\mathrm{~m}, 1 \mathrm{H}), 0.94(\mathrm{~s}, 3 \mathrm{H}), 0.84(\mathrm{~d}, J=6.4 \mathrm{~Hz}, 3 \mathrm{H}), 0.72(\mathrm{~d}, J=6.6 \mathrm{~Hz}, 3 \mathrm{H})$ ppm;

${ }^{13}$ C NMR (101 MHz, $\left.\mathrm{CDCl}_{3}\right): \delta=199.0,155.1,142.4,139.0,137.9,135.5,133.8$, 129.1 (2C), 128.5 (2C), 127.9 (2C), 127.8 (2C), 127.4, 126.5, 73.2, 68.5, 57.2, 47.4, $47.3,43.7,37.5,36.7,35.3,32.7,27.9,26.1,25.5,23.3,23.2,22.2,21.4,20.4$ ppm; HRMS (ESI): calculated for $\left[\mathrm{C}_{35} \mathrm{H}_{43} \mathrm{NO}_{4} \mathrm{~S}+\mathrm{H}\right]^{+}$574.2986, found 574.2983. 


\section{Compound 30}

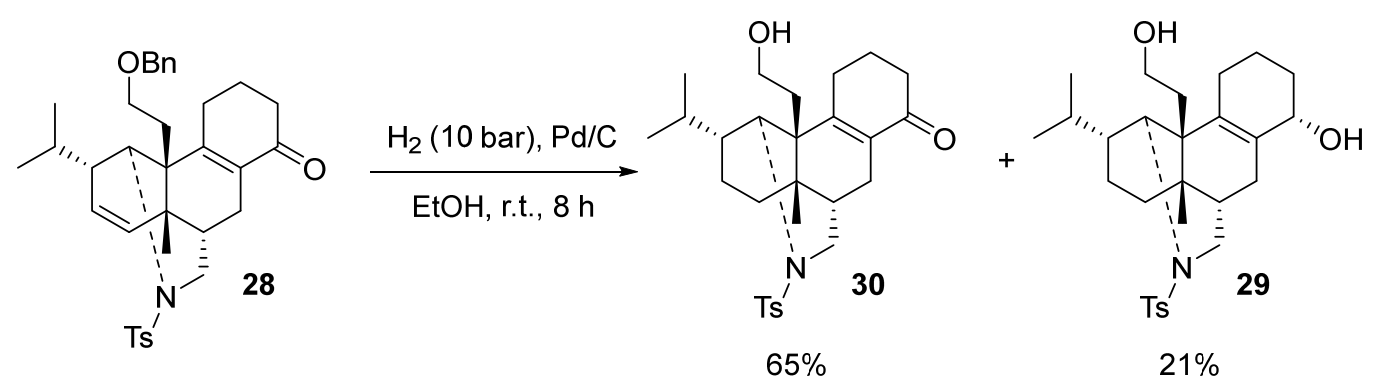

Palladium on carbon ( $40.0 \mathrm{mg}, 0.0377 \mathrm{mmol}, 10 \% \mathrm{Pd}$ on carbon, 0.27 equiv) was added to a degassed solution of compound 28 (80.0 $\mathrm{mg}, 0.139 \mathrm{mmol}, 1.0$ equiv) in EtOH $(15 \mathrm{~mL})$. The reactor was purged with $\mathrm{H}_{2}$ and the suspension was stirred under $\mathrm{H}_{2}$ (10 bar) for $8 \mathrm{~h}$. The reaction mixture was filtered through a plug of $\mathrm{SiO}_{2}$ topped with Celite (eluent: EtOAc). The filtrate was concentrated under reduced pressure. The residue was purified by flash chromatography on silica gel (eluent: EtOAc/PE, $1 / 3$ to $1 / 2)$ to give, in order of elution, compound 29 (14.3 $\mathrm{mg}$, white solid, yield: $21 \%)$ and compound 30 (44.0 mg, colorless oil, yield: 65\%).

Data for compound 30:

$\mathbf{R f}=0.25($ silica, $\mathrm{EtOAc} / \mathrm{PE}=1: 2)$

$[\alpha]_{\mathbf{D}}{ }^{20}=-22.5\left(c\right.$ 2.0, $\left.\mathrm{CHCl}_{3}\right)$;

${ }^{1} \mathbf{H}$ NMR $\left(400 \mathrm{MHz}, \mathrm{CDCl}_{3}\right): \delta=7.58(\mathrm{~d}, J=7.6 \mathrm{~Hz}, 2 \mathrm{H}), 7.21(\mathrm{~d}, J=7.7 \mathrm{~Hz}, 2 \mathrm{H})$, $4.20(\mathrm{~s}, 1 \mathrm{H}), 3.93-3.82(\mathrm{~m}, 1 \mathrm{H}), 3.69-3.50(\mathrm{~m}, 3 \mathrm{H}), 2.64-2.44(\mathrm{~m}, 3 \mathrm{H}), 2.44-2.27(\mathrm{~m}$, $4 \mathrm{H}), 2.38(\mathrm{~s}, 3 \mathrm{H}), 2.26-2.14(\mathrm{~m}, 1 \mathrm{H}), 2.02-1.84(\mathrm{~m}, 2 \mathrm{H}), 1.78-1.68(\mathrm{~m}, 2 \mathrm{H})$, 1.59-1.41 (m, 3H), 1.38-1.27 (m, 1H), 0.99 (s, 3H), 0.99-0.94 (m, 1H), $0.84(\mathrm{~s}, 3 \mathrm{H})$, $0.69(\mathrm{~d}, J=4.5 \mathrm{~Hz}, 3 \mathrm{H}) \mathrm{ppm}$;

${ }^{13} \mathrm{C}$ NMR (101 MHz, $\left.\mathrm{CDCl}_{3}\right): \delta=199.2,156.1,142.5,138.9,135.3,129.2(2 \mathrm{C})$, 126.5 (2C), 60.8, 56.9, 50.1, 47.5, 42.2, 38.7, 37.4, 35.9, 35.7, 33.3, 30.0, 29.6, 26.5, 26.4, 26.1, 22.3, 22.0, 21.4, $20.4 \mathrm{ppm}$;

HRMS (ESI): calculated for $\left[\mathrm{C}_{28} \mathrm{H}_{39} \mathrm{NO}_{4} \mathrm{~S}+\mathrm{H}\right]^{+}$486.2673, found 486.2669. 


\section{Compound 32}
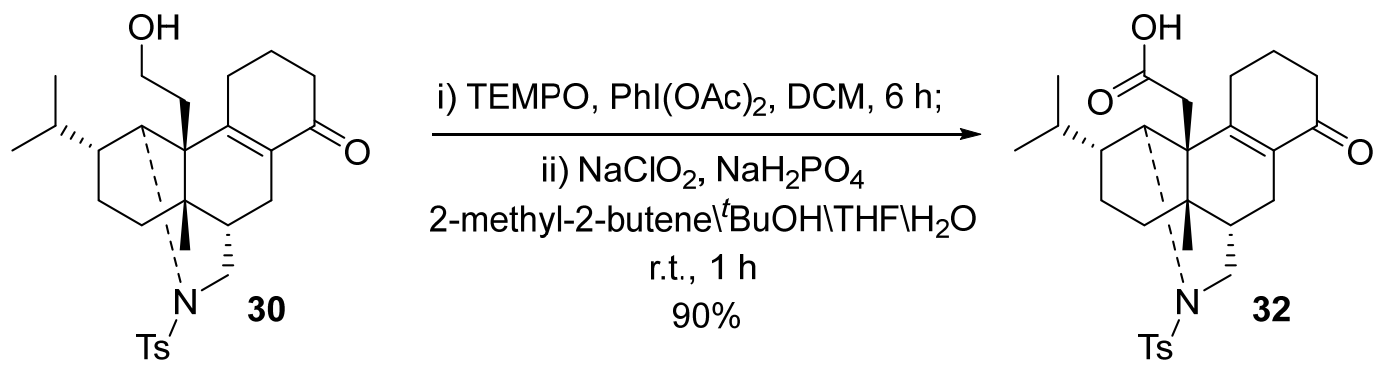

To a solution of compound 30 (48.9 mg, $0.101 \mathrm{mmol}, 1.0$ equiv) and $\mathrm{PhI}(\mathrm{OAc}) 2$ (36.5 $\mathrm{mg}, 0.113 \mathrm{mmol}, 1.1$ equiv) in DCM (2 mL), was added TEMPO (3.1 mg, 0.020 mmol, 0.2 equiv). The resulting mixture was stirred at room temperature for $6 \mathrm{~h}$. DCM was removed under reduced pressure and the residue was dissolved in THF (2 $\mathrm{mL})$ and ${ }^{t} \mathrm{BuOH}(2 \mathrm{~mL})$. 2-Methyl-2-butene (0.5 mL), $\mathrm{H}_{2} \mathrm{O}(0.8 \mathrm{~mL}), \mathrm{NaH}_{2} \mathrm{PO}_{4}(48.5$ mg, 0.404 mmol, 4.0 equiv) and $\mathrm{NaClO}_{2}(45.7 \mathrm{mg}, 0.404 \mathrm{mmol}, 80 \%$ purity, 4.0 equiv) were added. The resulting solution was stirred at room temperature for $1 \mathrm{~h}$, then $2 \mathrm{~N}$ $\mathrm{HCl}(2 \mathrm{~mL})$ and saturated aqueous $\mathrm{Na}_{2} \mathrm{SO}_{3}(1 \mathrm{~mL})$ were added. The resulting mixture was stirred at room temperature for $15 \mathrm{~min}$. The organic phase was separated and the aqueous phase was extracted with EtOAc $(4 \times 10 \mathrm{~mL})$. The combined organic phases were washed with brine, dried over anhydrous $\mathrm{Na}_{2} \mathrm{SO}_{4}$, filtered, and concentrated under reduced pressure. The residue was purified by flash chromatography on silica gel (eluent: EtOAc/PE, 2/1) to give compound 32 (45.6 mg, yield: 90\%) as a white solid.

$\mathbf{R f}=0.40($ silica, $\mathrm{EtOAc} / \mathrm{PE}=2: 1)$

$[\alpha]_{\mathbf{D}}^{\mathbf{2 0}}=-24.0\left(c\right.$ 1.0, $\left.\mathrm{CHCl}_{3}\right)$;

${ }^{1}$ H NMR (400 MHz, $\left.\mathrm{CDCl}_{3}\right): \delta=7.59(\mathrm{~d}, J=8.4 \mathrm{~Hz}, 2 \mathrm{H}), 7.21(\mathrm{~d}, J=8.4 \mathrm{~Hz}, 2 \mathrm{H})$, $4.71(\mathrm{~s}, 1 \mathrm{H}), 3.70(\mathrm{~d}, J=13.7 \mathrm{~Hz}, 1 \mathrm{H}), 3.60(\mathrm{dd}, J=13.7,3.6 \mathrm{~Hz}, 1 \mathrm{H}), 2.78(\mathrm{~d}, J=$ $17.4 \mathrm{~Hz}, 1 \mathrm{H}), 2.71(\mathrm{~d}, J=17.4 \mathrm{~Hz}, 1 \mathrm{H}), 2.67-2.59(\mathrm{~m}, 1 \mathrm{H}), 2.57-2.46(\mathrm{~m}, 2 \mathrm{H})$, 2.46-2.31 (m, 6H), 2.24-2.13 (m, 1H), 2.05-1.95 (m, 1H), 1.89-1.73 (m, 2H), $1.65-1.56(\mathrm{~m}, 2 \mathrm{H}), 1.53-1.45(\mathrm{~m}, 1 \mathrm{H}), 1.43-1.34(\mathrm{~m}, 1 \mathrm{H}), 0.99-0.92(\mathrm{~m}, 1 \mathrm{H}), 0.98(\mathrm{~s}$, $3 \mathrm{H}), 0.85$ (s, 3H), 0.69 (d, $J=6.0 \mathrm{~Hz}, 3 \mathrm{H}) \mathrm{ppm}$;

${ }^{13} \mathrm{C}$ NMR (101 MHz, $\left.\mathrm{CDCl}_{3}\right): \delta=199.5,177.2,155.8,142.5,139.0,134.2,129.2$, $126.5,54.8,49.9,47.1,42.2,38.5,37.2,35.4,34.8,33.5,30.3,29.5,26.5,26.0,25.6$, 
22.0, 21.8, 21.4, $20.4 \mathrm{ppm}$

HRMS (ESI): calculated for $\left[\mathrm{C}_{28} \mathrm{H}_{37} \mathrm{NO}_{5} \mathrm{~S}+\mathrm{H}\right]^{+}$500.2465, found 500.2457.

\section{Compound 29}

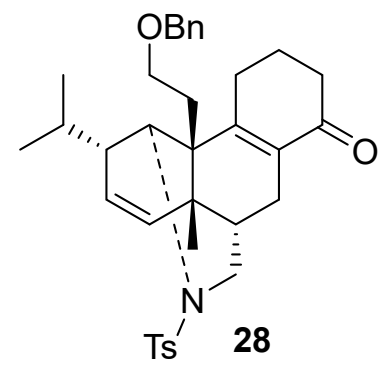
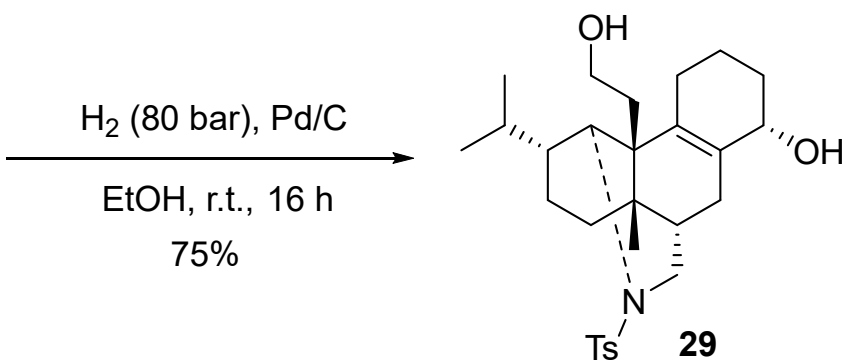

Palladium on carbon (140 mg, $0.132 \mathrm{mmol}, 10 \% \mathrm{Pd}$ on carbon, 0.27 equiv) was added to a degassed solution of compound 28 ( $280 \mathrm{mg}, 0.488 \mathrm{mmol}, 1.0$ equiv) in $\mathrm{EtOH}$ (50 $\mathrm{mL}$ ). The reactor was purged with $\mathrm{H}_{2}$ and the suspension was stirred under $\mathrm{H}_{2}$ (80 bar) for $16 \mathrm{~h}$. The reaction mixture was filtered through a plug of $\mathrm{SiO}_{2}$ topped with Celite (eluent: EtOAc). The filtrate was concentrated under reduced pressure. The residue was purified by flash chromatography on silica gel (eluent: EtOAc/PE, 1/3) to give compound 29 (179 mg, yield: 75\%) as a white solid.

$\mathbf{R f}=0.35$ (silica, $\mathrm{EtOAc} / \mathrm{PE}=1: 2)$;

$[\alpha]_{\mathrm{D}}^{20}=-56.0\left(c 0.5, \mathrm{CHCl}_{3}\right)$

mp: $181-184^{\circ} \mathrm{C}$;

${ }^{1}$ H NMR (400 MHz, $\left.\mathrm{CDCl}_{3}\right): \delta=7.60(\mathrm{~d}, J=8.1 \mathrm{~Hz}, 2 \mathrm{H}), 7.23(\mathrm{~d}, J=8.0 \mathrm{~Hz}, 2 \mathrm{H})$, $4.14(\mathrm{~d}, J=2.8 \mathrm{~Hz}, 1 \mathrm{H}), 3.93-3.79(\mathrm{~m}, 3 \mathrm{H}), 3.75(\mathrm{~d}, J=13.6 \mathrm{~Hz}, 1 \mathrm{H}), 3.65(\mathrm{td}, J=$ 10.1, $3.5 \mathrm{~Hz}, 1 \mathrm{H}), 3.55$ (dd, $J=13.6,4.3 \mathrm{~Hz}, 1 \mathrm{H}), 2.50$ (d, $J=17.9 \mathrm{~Hz}, 1 \mathrm{H}), 2.40$ (s, $3 \mathrm{H}), 2.37(\mathrm{~d}, J=17.9 \mathrm{~Hz}, 1 \mathrm{H}), 2.25-2.11(\mathrm{~m}, 2 \mathrm{H}), 1.97-1.84(\mathrm{~m}, 3 \mathrm{H}), 1.81-1.70(\mathrm{~m}$, 2H), 1.66-1.57 (m, 2H), 1.53-1.39 (m, 4H), 1.32-1.21 (m, 1H), 0.97 (d, $J=6.3 \mathrm{~Hz}$, $3 \mathrm{H}), 0.88(\mathrm{~s}, 3 \mathrm{H}), 0.85-0.78(\mathrm{~m}, 1 \mathrm{H}), 0.65(\mathrm{~d}, J=6.5 \mathrm{~Hz}, 3 \mathrm{H}) \mathrm{ppm}$;

${ }^{13} \mathrm{C}$ NMR (101 MHz, $\left.\mathrm{CDCl}_{3}\right): \delta=142.4,138.9,134.2,133.4,129.2(2 \mathrm{C}), 126.2(2 \mathrm{C})$, $67.1,60.9,56.2,50.3,45.6,42.0,39.3,35.8,35.4,33.8,33.4,31.5,30.3,26.4,26.2$, 26.0, 21.9, 21.4, 20.4, $17.3 \mathrm{ppm}$;

HRMS (ESI): calculated for $\left[\mathrm{C}_{28} \mathrm{H}_{41} \mathrm{NO}_{4} \mathrm{~S}+\mathrm{H}\right]^{+}$510.2649, found 510.2648. 


\section{Compound 36}
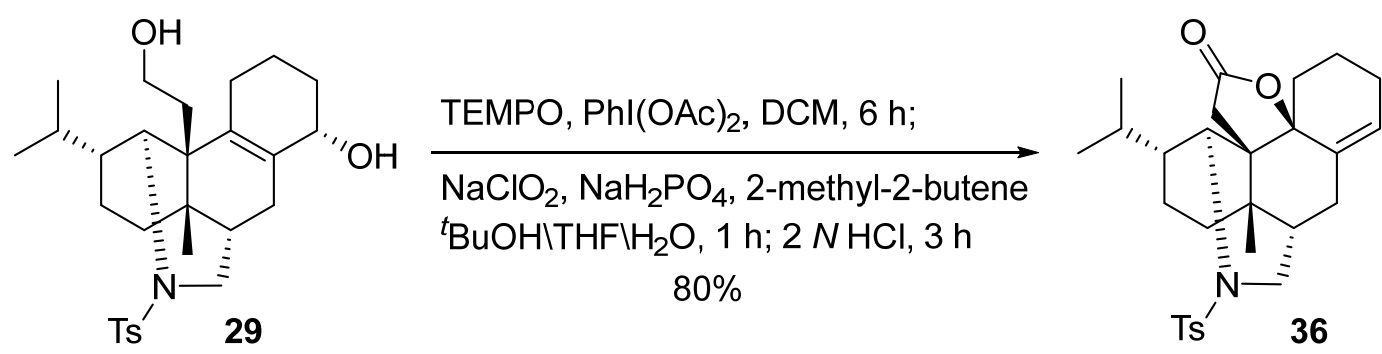

To a solution of compound 29 (160 mg, $0.328 \mathrm{mmol}, 1.0$ equiv) and $\mathrm{PhI}(\mathrm{OAc}) 2$ (116 $\mathrm{mg}, 0.361 \mathrm{mmol}, 1.1$ equiv) in DCM (10 mL), was added TEMPO (10.3 mg, 0.0656 mmol, 0.2 equiv). The resulting mixture was stirred at room temperature for $6 \mathrm{~h}$. DCM was removed under reduced pressure and the residue was dissolved in THF (7 $\mathrm{mL})$ and ${ }^{t} \mathrm{BuOH}(7 \mathrm{~mL})$. 2-Methyl-2-butene (1.5 mL), $\mathrm{H}_{2} \mathrm{O}(2.5 \mathrm{~mL}), \mathrm{NaH}_{2} \mathrm{PO}_{4}(157$ mg, $1.31 \mathrm{mmol}, 4.0$ equiv) and $\mathrm{NaClO}_{2}(148 \mathrm{mg}, 1.31 \mathrm{mmol}, 80 \%$ purity, 4.0 equiv) were added. The resulting solution was stirred at room temperature for $1 \mathrm{~h}$, then $2 \mathrm{~N}$ $\mathrm{HCl}(8 \mathrm{~mL})$ was added. The resulting mixture was stirred at room temperature for $3 \mathrm{~h}$. The organic phase was separated and the aqueous phase was extracted with EtOAc (4 $\times 15 \mathrm{~mL}$ ). The combined organic phases were washed with brine, dried over anhydrous $\mathrm{Na}_{2} \mathrm{SO}_{4}$, filtered, and concentrated under reduced pressure. The residue was purified by flash chromatography on silica gel (eluent: EtOAc/PE, 1/6) to give compound 36 (127 mg, yield: $80 \%$ ) as a white foam.

$\mathbf{R f}=0.55$ (silica, $\mathrm{EtOAc} / \mathrm{PE}=1: 4)$

$[\alpha]_{\mathrm{D}}{ }^{20}=-66.0\left(c \quad 0.5, \mathrm{CHCl}_{3}\right) ;$

${ }^{1} \mathbf{H}$ NMR $\left(400 \mathrm{MHz}, \mathrm{CDCl}_{3}\right): \delta=7.66(\mathrm{~d}, J=7.9 \mathrm{~Hz}, 2 \mathrm{H}), 7.28(\mathrm{~d}, J=7.9 \mathrm{~Hz}, 2 \mathrm{H})$, $5.51(\mathrm{~s}, 1 \mathrm{H}), 4.43(\mathrm{~s}, 1 \mathrm{H}), 3.78(\mathrm{dd}, J=12.5,5.3 \mathrm{~Hz}, 1 \mathrm{H}), 3.49(\mathrm{~d}, J=12.4 \mathrm{~Hz}, 1 \mathrm{H})$, 2.87 (d, $J=15.6 \mathrm{~Hz}, 1 \mathrm{H}), 2.61$ (d, $J=17.3 \mathrm{~Hz}, 1 \mathrm{H}), 2.46$ (d, $J=13.5 \mathrm{~Hz}, 1 \mathrm{H}), 2.44$ (s, 3H), $2.29(\mathrm{td}, J=13.1,3.3 \mathrm{~Hz}, 1 \mathrm{H}), 2.21-2.08(\mathrm{~m}, 2 \mathrm{H}), 2.05-1.95(\mathrm{~m}, 1 \mathrm{H}), 1.91(\mathrm{~d}, J$ $=15.8 \mathrm{~Hz}, 1 \mathrm{H}), 1.88-1.71(\mathrm{~m}, 4 \mathrm{H}), 1.63-1.56(\mathrm{~m}, 2 \mathrm{H}), 1.54-1.47(\mathrm{~m}, 1 \mathrm{H}), 1.37(\mathrm{dd}, J$ $=13.9,6.7 \mathrm{~Hz}, 1 \mathrm{H}), 1.12(\mathrm{~s}, 3 \mathrm{H}), 1.09(\mathrm{~d}, J=6.2 \mathrm{~Hz}, 3 \mathrm{H}), 1.05-0.98(\mathrm{~m}, 1 \mathrm{H}), 0.75(\mathrm{~d}$, $J=6.6 \mathrm{~Hz}, 3 \mathrm{H}) \mathrm{ppm}$;

${ }^{13} \mathrm{C} \mathrm{NMR}\left(101 \mathrm{MHz}, \mathrm{CDCl}_{3}\right): \delta=175.0,143.2,137.2,132.5,129.1$ (2C), 127.7 (2C), $127.3,86.8,55.8,49.7,49.5,48.0,39.6,37.4,37.1,36.6,33.9,30.1,28.3,25.8,25.4$, 
25.0, 21.8, 21.5, 20.5, $17.1 \mathrm{ppm}$;

HRMS (ESI): calculated for $\left[\mathrm{C}_{28} \mathrm{H}_{37} \mathrm{NO}_{4} \mathrm{~S}+\mathrm{H}\right]^{+} 484.2516$, found 484.2517.

\section{Compound 37}
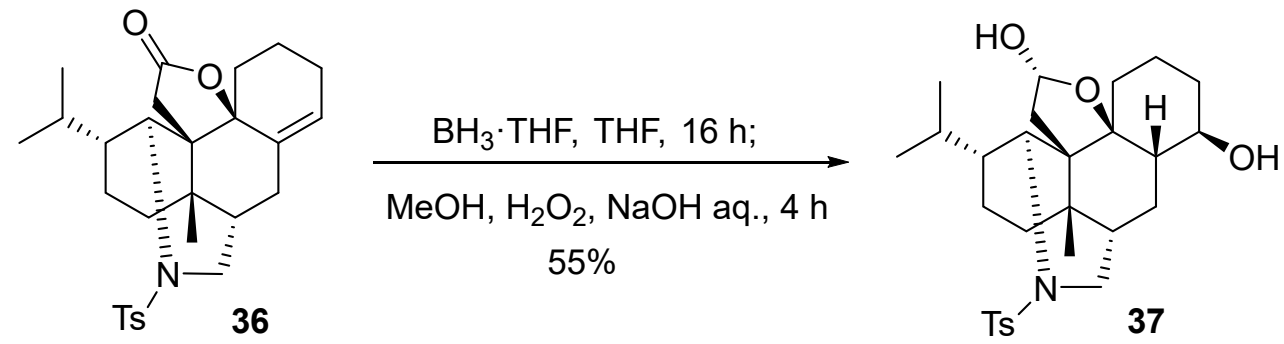

To a solution of compound 36 (110 $\mathrm{mg}, 0.227 \mathrm{mmol}, 1.0$ equiv) in THF (2 ml) was added a solution of $1.0 \mathrm{M} \mathrm{BH}_{3} \bullet \mathrm{THF}(2.27 \mathrm{~mL}, 2.27 \mathrm{mmol}, 10$ equiv) in THF at room temperature under argon. After being stirred for $16 \mathrm{~h}$ at room temperature, the reaction mixture was cooled to $0{ }^{\circ} \mathrm{C}, \mathrm{MeOH}(2 \mathrm{~mL})$ was added dropwise. After stirring for $5 \mathrm{~min}, \mathrm{H}_{2} \mathrm{O}_{2}(30 \%$ aq., $4.0 \mathrm{~mL})$ and $15 \% \mathrm{NaOH}$ aq. $(2.0 \mathrm{~mL})$ were added to the reaction mixture at the same temperature. The resulting mixture was warmed to room temperature and stirred for $4 \mathrm{~h}$. The reaction mixture was diluted with EtOAc $(20 \mathrm{ml})$ and $\mathrm{H}_{2} \mathrm{O}(10 \mathrm{ml})$. The organic phase was separated and the aqueous phase was extracted with EtOAc $(3 \times 15 \mathrm{~mL})$. The combined organic phases were washed with brine, dried over anhydrous $\mathrm{Na}_{2} \mathrm{SO}_{4}$, filtered, and concentrated under reduced pressure. The residue was purified by flash chromatography on silica gel (eluent: EtOAc/PE, 1/2 to 2/3) to give compound 37 (63.2 $\mathrm{mg}$, yield: 55\%) as a white solid.

$\mathbf{R f}=0.20($ silica, $\mathrm{EtOAc} / \mathrm{PE}=1: 1)$

$[\alpha]_{\mathbf{D}}^{\mathbf{2 0}}=-14.7\left(c 1.0, \mathrm{CHCl}_{3}\right)$;

${ }^{1}$ H NMR (400 MHz, $\left.\mathrm{CDCl}_{3}\right): \delta=7.68(\mathrm{~d}, J=8.1 \mathrm{~Hz}, 2 \mathrm{H}), 7.27(\mathrm{~d}, J=7.9 \mathrm{~Hz}, 2 \mathrm{H})$, 5.41-5.33 (m, 1H), $4.25(\mathrm{~s}, 1 \mathrm{H}), 3.75(\mathrm{dd}, J=12.4,3.8 \mathrm{~Hz}, 1 \mathrm{H}), 3.49-3.35(\mathrm{~m}, 2 \mathrm{H})$, $2.90(\mathrm{~d}, J=3.2 \mathrm{~Hz}, 1 \mathrm{H}), 2.49(\mathrm{dd}, J=14.2,6.4 \mathrm{~Hz}, 1 \mathrm{H}), 2.42(\mathrm{~s}, 3 \mathrm{H}), 2.28-2.20$ (m, 1H), 2.11-2.00 (m, 1H), 1.98-1.86 (m, 2H), 1.85-1.75 (m, 3H), 1.70-1.64 (m, 3H), $1.56-1.39(\mathrm{~m}, 2 \mathrm{H}), 1.36-1.13(\mathrm{~m}, 4 \mathrm{H}), 1.05$ (d, $J=5.6 \mathrm{~Hz}, 3 \mathrm{H}), 1.01(\mathrm{~s}, 3 \mathrm{H}), 0.65(\mathrm{~d}$, $J=6.1 \mathrm{~Hz}, 3 \mathrm{H}) \mathrm{ppm}$;

${ }^{13} \mathrm{C}$ NMR (101 MHz, $\left.\mathrm{CDCl}_{3}\right): \delta=143.0,138.1,129.2$ (2C), 127.4 (2C), 96.1, 89.0, 
71.6, 57.5, 54.0, 52.0, 47.1, 44.6, 44.2, 38.8, 37.0, 33.2, 29.8, 29.2, 29.0, 27.5, 27.3, 26.1, 22.0, 21.5, 20.5, $15.6 \mathrm{ppm}$;

HRMS (ESI): calculated for $\left[\mathrm{C}_{28} \mathrm{H}_{41} \mathrm{NO}_{5} \mathrm{~S}+\mathrm{Na}\right]^{+}$526.2598, found 526.2594.

\section{Compound 38}
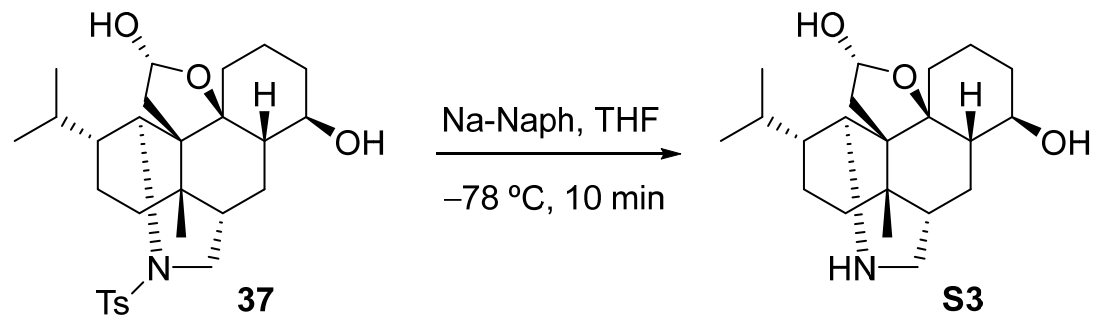

$$
\begin{gathered}
\underset{\mathrm{PPh}_{3}, \mathrm{CBr}_{4}, \mathrm{DMAP}, \mathrm{TEA}}{\longrightarrow} \\
\mathrm{DCM} \text {, r.t., } 12 \mathrm{~h} \\
50 \% \text { (2 steps) }
\end{gathered}
$$

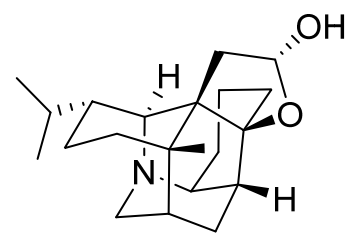

38

To a solution of naphthalene $(640 \mathrm{mg}, 5.00 \mathrm{mmol})$ in THF $(10 \mathrm{~mL})$ was added metal sodium (115 mg, $5.00 \mathrm{mmol})$ at room temperature. The mixture was stirred at room temperature for $1.5 \mathrm{~h}$ to yield a deep-blue sodium-naphthalenide solution $(0.5 \mathrm{M})$. The fresh-prepared sodium-naphthalenide solution was added slowly to a solution of compound 37 (45.2 $\mathrm{mg}, 0.0897 \mathrm{mmol}, 1.0$ equiv) in THF $(4 \mathrm{ml})$ at $-78{ }^{\circ} \mathrm{C}$ until the color of reaction mixture maintained unchanged blue (about $1.5 \mathrm{~mL}, 8$ equiv was used). After being stirred at $-78{ }^{\circ} \mathrm{C}$ for $10 \mathrm{~min}$, saturated aqueous $\mathrm{NH}_{4} \mathrm{Cl}(3 \mathrm{~mL})$ was added. The reaction mixture was warmed to room temperature, diluted with EtOAc $(20 \mathrm{ml})$ and poured into $\mathrm{H}_{2} \mathrm{O}(10 \mathrm{ml})$. The organic phase was separated and the aqueous phase was extracted with EtOAc $(3 \times 20 \mathrm{~mL})$. The combined organic phases were washed with brine, dried over anhydrous $\mathrm{Na}_{2} \mathrm{SO}_{4}$, filtered, and concentrated under reduced pressure. The residue was purified by flash chromatography on silica gel (eluent: EtOAc/PE, 1/10, then TEA/MeOH/DCM, 1/5/100) to give compound $\mathbf{S 3}$ (35.5 mg, yield: $>100 \%$ ) as a pale yellow oil. The material was used directly for the next step without further purification.

To a cooled $\left(0^{\circ} \mathrm{C}\right)$ solution of the abovementioned compound $\mathbf{S 3}$ (35.5 mg), DMAP 
(2.2 mg, 0.018 mmol, 0.2 equiv) and $\mathrm{PPh}_{3}(35.4 \mathrm{mg}, 0.135 \mathrm{mmol}, 1.5$ equiv) in DCM $(2 \mathrm{~mL})$ under argon atmosphere, TEA $\left(0.038 \mathrm{~mL}, 0.27 \mathrm{mmol}, 3.0\right.$ equiv) and $\mathrm{CBr}_{4}$ (44.8 mg, $0.135 \mathrm{mmol}, 1.5$ equiv) in DCM $(0.5 \mathrm{~mL})$ were added. After being stirred at room tempereture for $12 \mathrm{~h}$, a saturated aqueous $\mathrm{NaHCO}_{3}(5 \mathrm{~mL})$ was added. The organic phase was separated and the aqueous phase was extracted with DCM $(3 \times 5$ $\mathrm{mL})$. The combined organic phases were washed with brine, dried over anhydrous $\mathrm{Na}_{2} \mathrm{SO}_{4}$, filtered, and concentrated under reduced pressure. The residue was purified by flash chromatography on silica gel (eluent: $\mathrm{MeOH} / \mathrm{DCM}, 1 / 100$, then TEA/MeOH/DCM, 1/4/100) to give compound 38 (15.0 mg, yield: 50\%) as pale yellow solid.

$\mathbf{R f}=0.35($ silica, $\mathrm{MeOH} / \mathrm{DCM}=1: 20)$;

$[\alpha]_{\mathbf{D}}{ }^{20}=-40.0\left(c \quad 0.5, \mathrm{CHCl}_{3}\right)$

mp: $189-194^{\circ} \mathrm{C}$;

${ }^{1} \mathbf{H}$ NMR (400 MHz, $\left.\mathrm{CDCl}_{3}\right): \delta=5.53(\mathrm{t}, J=5.5 \mathrm{~Hz}, 1 \mathrm{H}), 3.70(\mathrm{dt}, J=14.1,2.9 \mathrm{~Hz}$, $1 \mathrm{H}), 3.53(\mathrm{~d}, J=4.5 \mathrm{~Hz}, 1 \mathrm{H}), 3.36-3.25(\mathrm{~m}, 2 \mathrm{H}), 2.76(\mathrm{~d}, J=14.0 \mathrm{~Hz}, 1 \mathrm{H}), 2.52-2.43$ (m, 1H), $2.37(\mathrm{dq}, J=13.2,3.0 \mathrm{~Hz}, 1 \mathrm{H}), 2.27-2.19(\mathrm{~m}, 1 \mathrm{H}), 2.03-1.97(\mathrm{~m}, 1 \mathrm{H})$, $1.88-1.53(\mathrm{~m}, 12 \mathrm{H}), 1.42(\mathrm{dd}, J=14.1,8.2 \mathrm{~Hz}, 1 \mathrm{H}), 0.99$ (s, 3H), 0.98 (d, $J=6.5 \mathrm{~Hz}$, $3 \mathrm{H}), 0.88(\mathrm{~d}, J=6.7 \mathrm{~Hz}, 3 \mathrm{H}) \mathrm{ppm}$;

${ }^{13} \mathrm{C}$ NMR (101 MHz, $\left.\mathrm{CDCl}_{3}\right): \delta=98.7,87.5,65.5,57.3,56.4,50.4,43.7,38.9,37.8$, 36.8, 36.7 (2C), 36.2, 30.9, 30.3, 29.3, 29.0, 27.8, 21.2, 21.1, 20.9 ppm;

HRMS (ESI): calculated for $\left[\mathrm{C}_{21} \mathrm{H}_{33} \mathrm{NO}_{2}+\mathrm{H}\right]^{+} 332.2584$, found 332.2580. 


\section{Dapholdhamine B (1)}

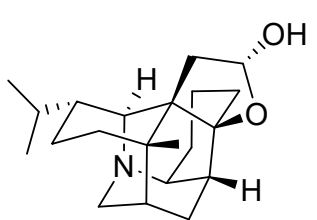

38

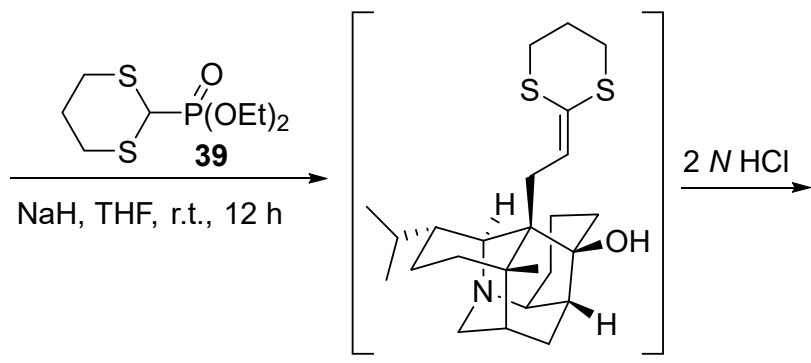

40

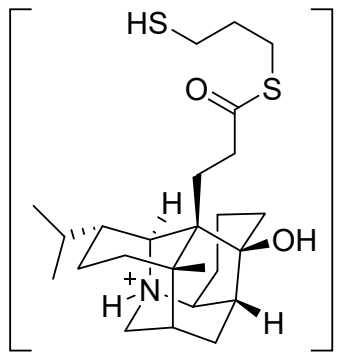

41

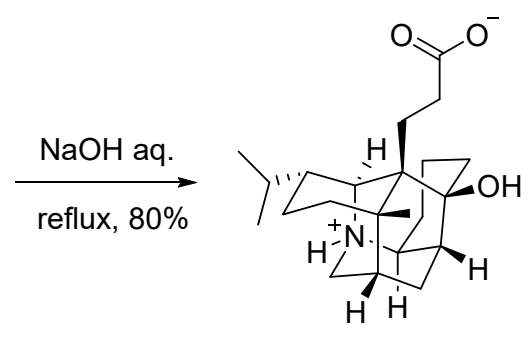

Dapholdhamine B, 1

To a solution of compound 38 (11.2 $\mathrm{mg}, 0.0338 \mathrm{mmol}, 1.0$ equiv) and diethyl (1,3-dithian-2-yl) phosphonate (39) (34.6 mg, $0.135 \mathrm{mmol}, 4$ equiv) in THF (2 mL) under argon atmosphere, $\mathrm{NaH}(8.1 \mathrm{mg}, 0.20 \mathrm{mmol}, 60 \mathrm{wt} \%$ dispersion in paraffin, 6 equiv) was added. Sonication was applied to the mixture at $25{ }^{\circ} \mathrm{C}$ for $5 \mathrm{~min}$. After being stirred at room tempereture for $12 \mathrm{~h}, 2 \mathrm{~N} \mathrm{HCl}(2 \mathrm{~mL})$ was added dropwise. The resulting mixture was warmed to refluxing and stirred for $6 \mathrm{~h}$. The reaction mixture was cooled to room temperature and THF was removed under reduced pressure. $\mathrm{MeOH}(2 \mathrm{~mL})$ and $15 \% \mathrm{NaOH}$ aq. $(2 \mathrm{~mL})$ were added to the residue. The resulting mixture was warmed to refluxing and stirred for $8 \mathrm{~h}$. The reaction mixture was cooled to room temperature and $\mathrm{H}_{2} \mathrm{O}(10 \mathrm{~mL})$ was added. A pH level of 12 was obtained by adding $2 N \mathrm{HCl}(\sim 2 \mathrm{~mL})$ and the mixture was extracted with $\mathrm{CHCl}_{3}(3 \times 5 \mathrm{~mL})$. The aqueous phase was adjusted to neutral $(\mathrm{pH}=7.0-7.5)^{a}$ by adding $2 N \mathrm{HCl}(\sim 0.5 \mathrm{~mL})$ and the solution was extracted with $\mathrm{CHCl}_{3}(2 \times 5 \mathrm{~mL})$. Freeze drying method was used to freeze-dry the aqueous phase. The residue was purified by flash chromatography on silica gel (eluent: $\mathrm{MeOH} / \mathrm{CHCl}_{3}, 1 / 20$ to $\left.1 / 5\right)$ give compound 1 (9.8 mg, yield: $80 \%$ ) as a white solid.

$\mathbf{R f}=0.2($ silica $, \mathrm{MeOH} / \mathrm{DCM}=1: 10)$; 
$[\alpha]_{\mathbf{D}}{ }^{20}=+6.67(c 0.3, \mathrm{MeOH}) ;$

${ }^{1} \mathbf{H}$ NMR $\left(400 \mathrm{MHz}, \mathrm{CDCl}_{3} / \mathrm{CD}_{3} \mathrm{OD}=2: 1\right): \delta=3.81-3.67(\mathrm{~m}, 2 \mathrm{H}), 3.48(\mathrm{t}, J=2.2$ Hz, 1H), 2.91 (d, $J=13.2 \mathrm{~Hz}, 1 \mathrm{H}), 2.45$ (dd, $J=13.3,3.0 \mathrm{~Hz}, 1 \mathrm{H}), 2.26$ (dt, $J=14.6$, $4.6 \mathrm{~Hz}, 1 \mathrm{H}), 2.17-2.02(\mathrm{~m}, 3 \mathrm{H}), 1.90-1.64(\mathrm{~m}, 6 \mathrm{H}), 1.61-1.49(\mathrm{~m}, 3 \mathrm{H}), 1.41-1.14(\mathrm{~m}$, $6 \mathrm{H}), 1.00(\mathrm{~s}, 3 \mathrm{H}), 0.77(\mathrm{~d}, J=3.2 \mathrm{~Hz}, 3 \mathrm{H}), 0.76(\mathrm{~d}, J=3.2 \mathrm{~Hz}, 3 \mathrm{H}) \mathrm{ppm}$;

${ }^{13} \mathrm{C}$ NMR (101 MHz, $\left.\mathrm{CDCl}_{3} / \mathrm{CD}_{3} \mathrm{OD}=2: 1\right): \delta=180.0,72.6,64.8,57.5,54.3,44.5$, $40.3,39.2,37.7,37.6,37.6,35.6,32.9,29.7,27.6,26.1,25.2,25.1,23.2,21.2,20.0$, 19.9 ppm;

HRMS (ESI): calculated for $\left[\mathrm{C}_{22} \mathrm{H}_{35} \mathrm{NO}_{3}+\mathrm{H}\right]^{+} 362.2690$, found 362.2686.

${ }^{a}$ Note: We observed the NMR signals of the isolated material to drift depending upon the $\mathrm{pH}$ level (see next page). We found when the $\mathrm{pH}<5.5$, the isolated material contained the lactone $\mathbf{4 2}$ (by MS). 

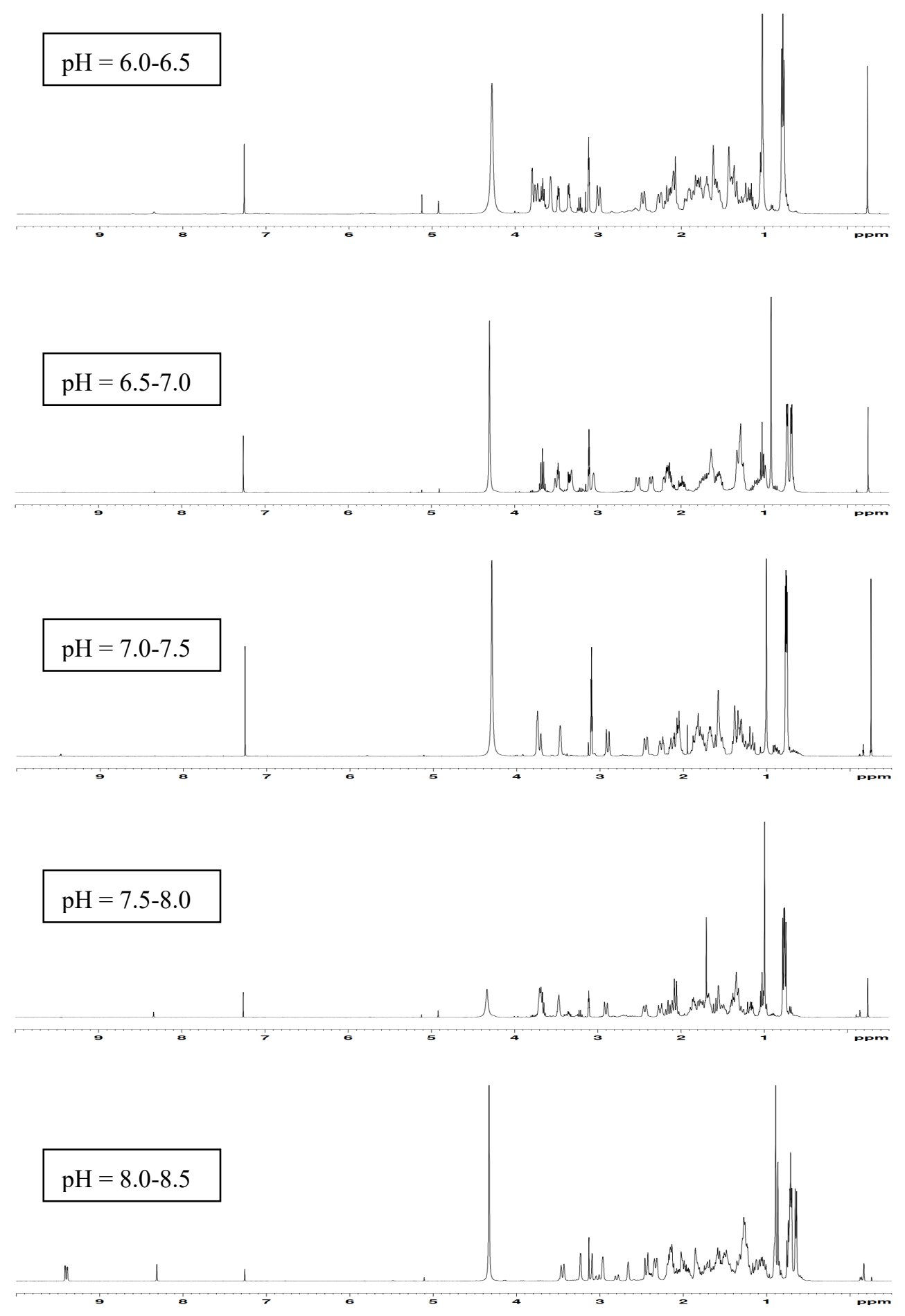

Reported ${ }^{1} \mathrm{H} \mathrm{NMR}^{4}$ 
1H NMR comparison of Dapholdhamine B with reported values

\begin{tabular}{|c|c|}
\hline $\begin{array}{c}\text { Hao's data }^{4} \\
\left(400 \mathrm{MHz}, \mathrm{CDCl}_{3} / \mathrm{CD}_{3} \mathrm{OD}=2: 1\right)\end{array}$ & $\begin{array}{c}\text { Our synthetic data } \\
\left(400 \mathrm{MHz}, \mathrm{CDCl}_{3} / \mathrm{CD}_{3} \mathrm{OD}=2: 1\right) \\
(\mathrm{pH}=7.0-7.5)\end{array}$ \\
\hline $\begin{array}{c}3.91(\mathrm{~d}, J=12.8 \mathrm{~Hz}, 1 \mathrm{H}) \\
3.89(\mathrm{~d}, J=6.4 \mathrm{~Hz}, 1 \mathrm{H})\end{array}$ & $3.81-3.67(\mathrm{~m}, 2 \mathrm{H})$ \\
\hline $3.45(\mathrm{br} \mathrm{d}, J=12.8 \mathrm{~Hz}, 1 \mathrm{H})$ & $3.48(\mathrm{t}, J=2.2 \mathrm{~Hz}, 1 \mathrm{H})$ \\
\hline $3.35(\mathrm{~d}, J=3.2 \mathrm{~Hz}, 1 \mathrm{H})$ & $2.91(\mathrm{~d}, J=13.2 \mathrm{~Hz}, 1 \mathrm{H})$ \\
\hline $2.63(\mathrm{~m}, 1 \mathrm{H})$ & $2.45(\mathrm{dd}, J=13.3,3.0 \mathrm{~Hz}, 1 \mathrm{H})$ \\
\hline $2.32(\mathrm{~m}, 1 \mathrm{H})$ & $2.26(\mathrm{dt}, J=14.6,4.6 \mathrm{~Hz}, 1 \mathrm{H})$ \\
\hline $\begin{array}{l}2.29(\mathrm{~m}, 1 \mathrm{H}) \\
2.28(\mathrm{~m}, 1 \mathrm{H}) \\
2.16(\mathrm{~m}, 1 \mathrm{H})\end{array}$ & $2.17-2.02(\mathrm{~m}, 3 \mathrm{H})$ \\
\hline $\begin{array}{l}2.06(\mathrm{~m}, 1 \mathrm{H}) \\
2.04(\mathrm{~m}, 1 \mathrm{H}) \\
1.92(\mathrm{~m}, 2 \mathrm{H}) \\
1.80(\mathrm{~m}, 2 \mathrm{H})\end{array}$ & $1.90-1.64(\mathrm{~m}, 6 \mathrm{H})$ \\
\hline $\begin{array}{l}1.70(\mathrm{~m}, 1 \mathrm{H}) \\
1.60(\mathrm{~m}, 1 \mathrm{H}) \\
1.58(\mathrm{~m}, 1 \mathrm{H})\end{array}$ & $1.61-1.49(\mathrm{~m}, 3 \mathrm{H})$ \\
\hline $\begin{array}{l}1.57(\mathrm{~m}, 1 \mathrm{H}) \\
1.48(\mathrm{~m}, 1 \mathrm{H}) \\
1.41(\mathrm{~m}, 1 \mathrm{H}) \\
1.39(\mathrm{~m}, 1 \mathrm{H}) \\
1.38(\mathrm{~m}, 1 \mathrm{H}) \\
1.33(\mathrm{~m}, 1 \mathrm{H})\end{array}$ & $1.41-1.14(\mathrm{~m}, 6 \mathrm{H})$ \\
\hline $1.00(\mathrm{~d}, J=5.1 \mathrm{~Hz}, 3 \mathrm{H})$ & $0.77(\mathrm{~d}, J=3.2 \mathrm{~Hz}, 3 \mathrm{H})$ \\
\hline $0.93(\mathrm{~d}, J=5.0 \mathrm{~Hz}, 3 \mathrm{H})$ & $0.76(\mathrm{~d}, J=3.2 \mathrm{~Hz}, 3 \mathrm{H})$ \\
\hline
\end{tabular}


${ }^{13}$ C NMR comparison of Dapholdhamine B with reported values

\begin{tabular}{|c|c|}
\hline $\begin{array}{c}\text { Hao's data }^{4} \\
\left(101 \mathrm{MHz}, \mathrm{CDCl}_{3} / \mathrm{CD}_{3} \mathrm{OD}=2: 1\right)\end{array}$ & $\begin{array}{c}\text { Our synthetic data } \\
\left(101 \mathrm{MHz}, \mathrm{CDCl}_{3} / \mathrm{CD}_{3} \mathrm{OD}=2: 1\right) \\
(\mathrm{pH}=7.0-7.5)\end{array}$ \\
\hline 179.1 & 180.0 \\
\hline 80.5 & 72.6 \\
\hline 66.8 & 64.8 \\
\hline 64.4 & 57.5 \\
\hline 48.2 & 54.3 \\
\hline 47.1 & 44.5 \\
\hline 45.0 & 40.3 \\
\hline 38.3 & 39.2 \\
\hline 37.3 & 37.7 \\
\hline 36.3 & 37.6 \\
\hline 35.0 & 37.6 \\
\hline 34.5 & 35.6 \\
\hline 34.1 & 32.9 \\
\hline 31.3 & 29.7 \\
\hline 29.8 & 27.6 \\
\hline 28.4 & 26.1 \\
\hline 26.8 & 25.2 \\
\hline 25.6 & 25.1 \\
\hline 24.6 & 23.2 \\
\hline 24.5 & 21.2 \\
\hline 21.1 & 20.0 \\
\hline 20.5 & 19.9 \\
\hline
\end{tabular}




\section{Dapholdhamine B lactone (42)}

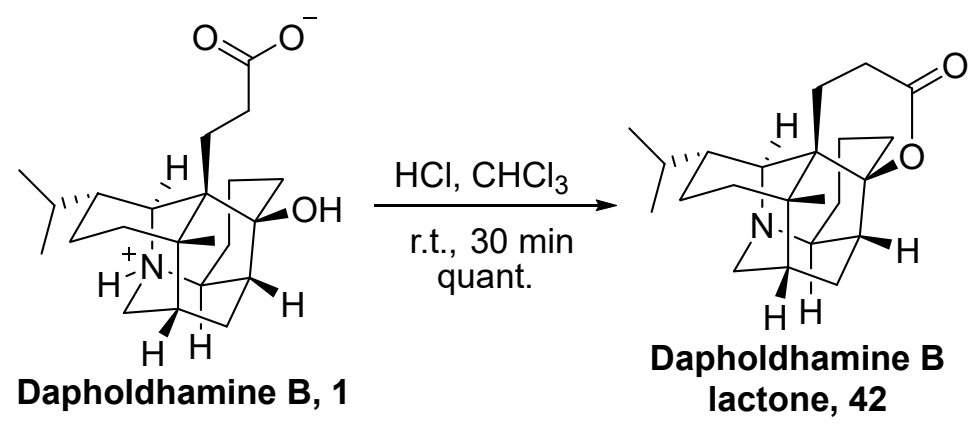

To a suspension of compound 1 ( $7.0 \mathrm{mg}, 0.019 \mathrm{mmol}, 1.0$ equiv) in $\mathrm{CHCl}_{3}$ (4 mL) was added dropwise concentrated hydrochloric acid $(0.50 \mathrm{~mL})$. After being stirred at room tempereture for $30 \mathrm{~min}$, saturated aqueous $\mathrm{NaHCO}_{3}(5 \mathrm{~mL})$ and $15 \% \mathrm{NaOH}$ aq. $(2 \mathrm{~mL})$ were added dropwise. The resulting mixture was stirred at room temperature for $30 \mathrm{~min}$. The organic phase was separated and the aqueous phase was extracted with DCM $(3 \times 10 \mathrm{~mL})$. The combined organic phases were washed with brine, dried over anhydrous $\mathrm{Na}_{2} \mathrm{SO}_{4}$, filtered, and concentrated under reduced pressure to give compound 42 (6.7 mg, yield: 100\%) as a pale yellow oil.

$\mathbf{R f}=0.45($ silica $, \mathrm{MeOH} / \mathrm{DCM}=1: 20)$;

$[\alpha]_{\mathbf{D}}{ }^{\mathbf{2 0}}=-30.0\left(c 0.5, \mathrm{CHCl}_{3}\right)$;

${ }^{1} \mathbf{H}$ NMR (400 MHz, $\left.\mathrm{CDCl}_{3}\right): \delta=3.71(\mathrm{dt}, J=14.1,3.1 \mathrm{~Hz}, 1 \mathrm{H}), 3.39(\mathrm{~d}, J=7.7 \mathrm{~Hz}$, $1 \mathrm{H}), 3.18(\mathrm{~d}, J=4.6 \mathrm{~Hz}, 1 \mathrm{H}), 2.80(\mathrm{~d}, J=14.1 \mathrm{~Hz}, 1 \mathrm{H}), 2.64(\mathrm{dd}, J=6.0,2.9 \mathrm{~Hz}, 1 \mathrm{H})$, $2.62(\mathrm{dd}, J=6.0,2.9 \mathrm{~Hz}, 1 \mathrm{H}), 2.54(\mathrm{ddd}, J=13.4,5.9,3.2 \mathrm{~Hz}, 1 \mathrm{H}), 2.30-2.21(\mathrm{~m}$, 1H), 2.08-1.99 (m, 1H), 1.99-1.90 (m, 1H), 1.89-1.73 (m, 6H), 1.70-1.52 (m, 5H), 1.46-1.37 (m, 1H), 1.27-1.19 (m, 2H), 1.00 (s, 3H), 0.95 (d, J=6.4 Hz, 3H), 0.88 (d, $J=6.6 \mathrm{~Hz}, 3 \mathrm{H}) \mathrm{ppm}$;

${ }^{13}$ C NMR (101 MHz, $\left.\mathrm{CDCl}_{3}\right): \delta=172.7,85.5,63.0,57.9,55.8,40.3,39.7,38.1,37.6$, $36.4,36.0,34.8,30.9,29.7,28.5,27.7,27.6,27.0,21.6,21.1,20.8,20.6$ ppm;

HRMS (ESI): calculated for $\left[\mathrm{C}_{22} \mathrm{H}_{33} \mathrm{NO}_{2}+\mathrm{H}\right]^{+}$344.2584, found 344.2582.

Through extensive NMR analysis, we clearly assigned the chemical structure of $\mathbf{4 2}$, see Figures S1, S2 and Table S1. 


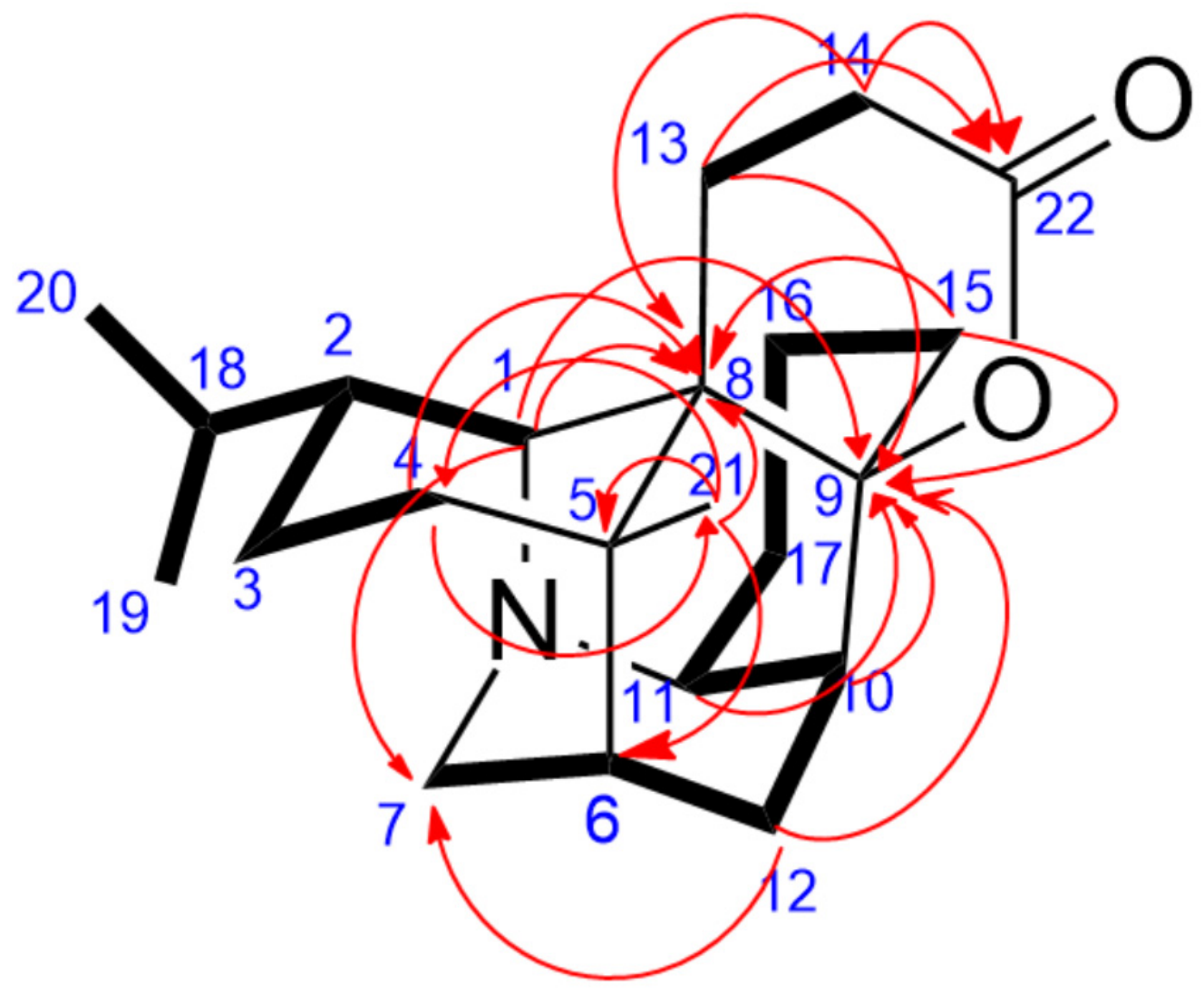

Figure S1. ${ }^{1} \mathrm{H}-{ }^{1} \mathrm{H}$ COSY (bold) and key HMBC (arrow, $\mathrm{H} \rightarrow \mathrm{C}$ ) correlations of dapholdhamine B lactone (42)

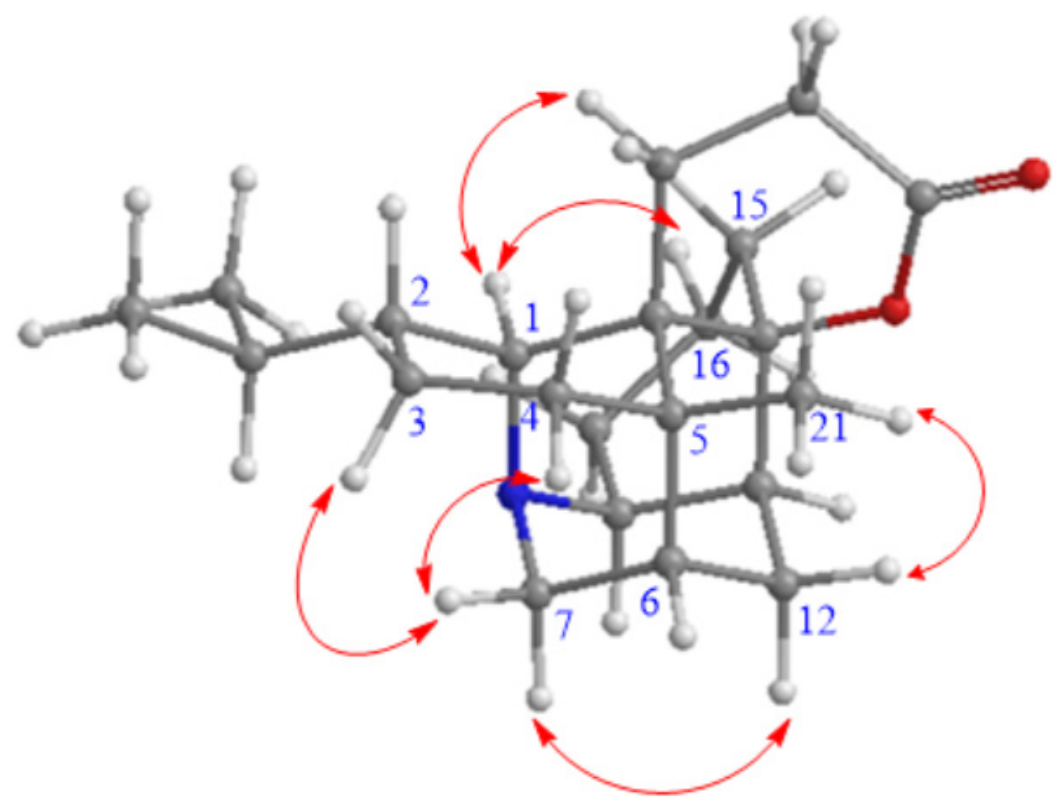

Figure S2. Key NOESY (red) correlations of dapholdhamine B lactone (42) 
Table S1. ${ }^{1} \mathrm{H}$ and ${ }^{13} \mathrm{C}$ NMR Data of dapholdhamine B lactone (42) in $\mathrm{CDCl}_{3}$

\begin{tabular}{|c|c|c|c|}
\hline Position & $\mathrm{H}(J, \mathrm{~Hz})$ & $\mathrm{C}$ & $\operatorname{HMBC}\left({ }^{1} \mathrm{H}\right)$ \\
\hline 1 & 3.18 & 57.86 & $3 \mathrm{~b}, 7 \mathrm{a}, 11,13 \mathrm{a}, 18$ \\
\hline 2 & 1.25 & 39.69 & $1,4 \mathrm{a}, 19,20$ \\
\hline $3 a$ & 1.60 & 26.95 & $1,4 \mathrm{a}$ \\
\hline $3 b$ & $1.84^{a}$ & & \\
\hline $4 a$ & 1.43 & 36.42 & 21 \\
\hline $4 b$ & $1.84^{a}$ & & \\
\hline 5 & & 37.58 & $1,4 \mathrm{a}, 7 \mathrm{a}, 21$ \\
\hline 6 & 1.21 & 38.11 & $4 a, 21$ \\
\hline $7 a$ & 2.80 & 55.77 & $1,12 b$ \\
\hline $7 b$ & 3.71 & & \\
\hline 8 & & 40.26 & $1,4 a, 14 b, 15 a, 21$ \\
\hline 9 & & 85.53 & $1,10,11,12 a, 12 b, 13 b, 15 a, 15 b$ \\
\hline 10 & 1.87 & 35.98 & $15 \mathrm{a}$ \\
\hline 11 & 3.38 & 63.01 & $1,7 \mathrm{a}, 7 \mathrm{~b}, 10,15 \mathrm{a}, 17 \mathrm{a}, 17 \mathrm{~b}$ \\
\hline $12 \mathrm{a}$ & 1.61 & 29.72 & $4 a, 7 a, 7 b$ \\
\hline $12 b$ & 2.55 & & \\
\hline $13 \mathrm{a}$ & 1.56 & 21.57 & $14 a, 14 b$ \\
\hline $13 b$ & 2.04 & & \\
\hline $14 \mathrm{a}$ & 2.62 & 28.45 & $13 b$ \\
\hline $14 b$ & 2.64 & & \\
\hline $15 \mathrm{a}$ & 2.25 & 34.83 & $17 \mathrm{a}$ \\
\hline $15 b$ & 1.78 & & \\
\hline $16 \mathrm{a}$ & $1.83^{a}$ & 20.59 & $11,15 a, 15 b, 17$ \\
\hline $16 b$ & $1.83^{a}$ & & \\
\hline $17 \mathrm{a}$ & 1.96 & 27.56 & $11,15 \mathrm{a}$ \\
\hline $17 b$ & 1.67 & & \\
\hline 18 & 1.59 & 30.90 & 19,20 \\
\hline 19 & 0.88 & 20.85 & 20 \\
\hline 20 & 0.95 & 21.08 & 19 \\
\hline 21 & 1.00 & 27.73 & $4 a, 7 a$ \\
\hline 22 & & 172.67 & $13 a, 13 b, 14 a, 14 b$ \\
\hline
\end{tabular}

${ }^{a}$ overlapped 


\section{HPLC chromatograms:}

$\mathrm{mAU}$

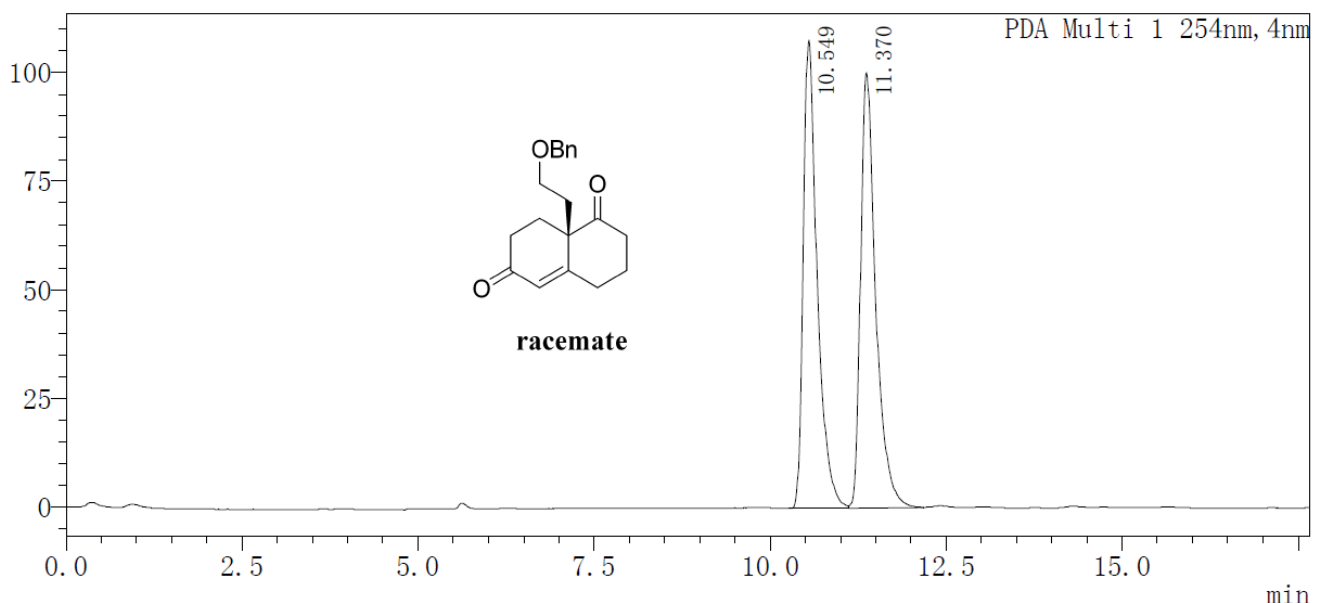

Figure S3. HPLC analysis of racemic 5

\begin{tabular}{|c|c|c|c|c|}
\hline $\mathrm{Pk} \#$ & Retention Time & Area & Height & Area \% \\
\hline 1 & 10.549 & 1489737 & 107814 & 49.916 \\
\hline 2 & 11.370 & 1494758 & 100291 & 50.084 \\
\hline
\end{tabular}

Conditions: Chiralpak AD-3, column temperature: $30^{\circ} \mathrm{C}, n$-hexane/ isopropanol $=85 /$ 15 , flow $0.8 \mathrm{~mL} / \mathrm{min}$, detection at $254 \mathrm{~nm}$.

$\mathrm{mAU}$

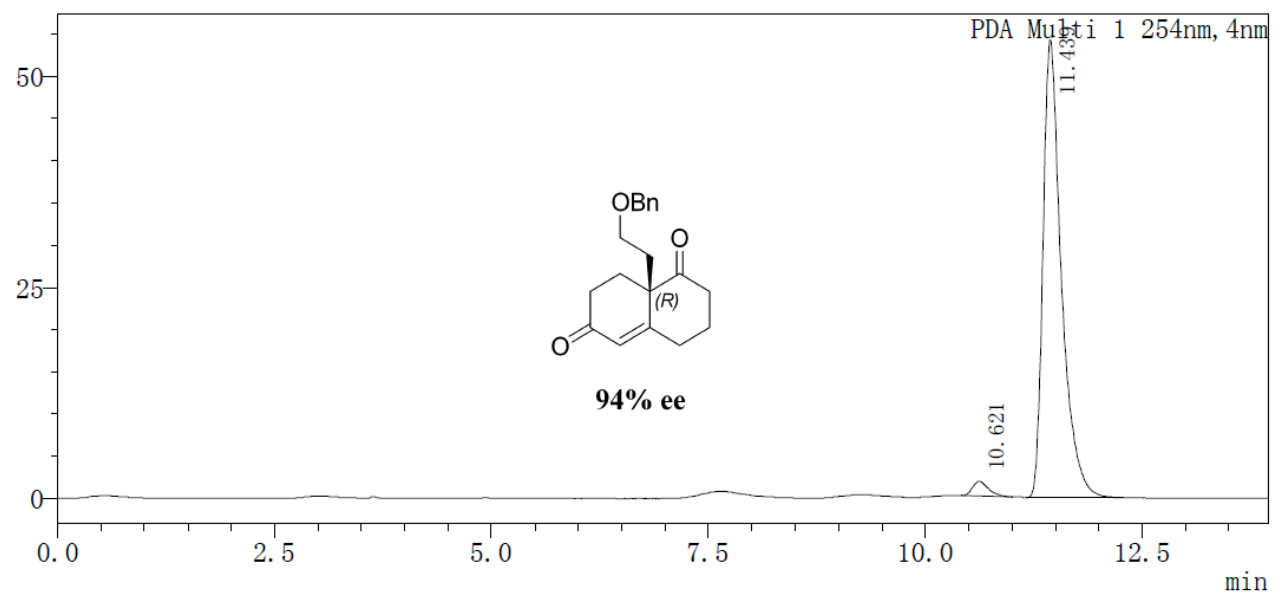

Figure S4. HPLC analysis of $(\boldsymbol{R})-\mathbf{5}$

\begin{tabular}{|c|c|c|c|c|}
\hline Pk\# & Retention Time & Area & Height & Area \% \\
\hline 1 & 10.621 & 22108 & 1761 & 2.695 \\
\hline 2 & 11.439 & 798368 & 54211 & 97.305 \\
\hline
\end{tabular}

Conditions: Chiralpak AD-3, column temperature: $30^{\circ} \mathrm{C}, n$-hexane/ isopropanol $=85$ / 15 , flow $0.8 \mathrm{~mL} / \mathrm{min}$, detection at $254 \mathrm{~nm}$. 


\section{Crystallographic data}

Crystal Data for Compound $\mathbf{1 6}\left(\mathrm{C}_{36} \mathrm{H}_{41} \mathrm{NO}_{7} \mathrm{~S}\right)$ :

\begin{tabular}{llll} 
Bond precision: & $\mathrm{C}-\mathrm{C}=0.0054 \mathrm{~A}$ & \multicolumn{2}{c}{ Wavelength $=0.71073$} \\
Cell: & $\mathrm{a}=38.5549(16)$ & $\mathrm{b}=38.5549(16)$ & $\mathrm{c}=14.8445(7)$ \\
& alpha $=90$ & beta $=90$ & gamma $=120$
\end{tabular}

Temperature: $\quad 100 \mathrm{~K}$

\begin{tabular}{|c|c|c|}
\hline & Calculated & Reported \\
\hline Volume & $19110(2)$ & $19109.8(18$ \\
\hline Space group & P 62 & P 62 \\
\hline Hall group & P 62 & P 62 \\
\hline Moiety formula & $\mathrm{C}_{36} \mathrm{H}_{41} \mathrm{~N} \mathrm{O}_{7} \mathrm{~S}$ & $\mathrm{C}_{36} \mathrm{H}_{41} \mathrm{~N} \mathrm{O}_{7} \mathrm{~S}$ \\
\hline Sum formula & $\mathrm{C}_{36} \mathrm{H}_{41} \mathrm{~N} \mathrm{O}_{7} \mathrm{~S}$ & $\mathrm{C}_{36} \mathrm{H}_{41} \mathrm{~N} \mathrm{O}_{7} \mathrm{~S}$ \\
\hline $\mathrm{Mr}$ & 631.76 & 631.76 \\
\hline $\mathrm{Dx}, \mathrm{g} \mathrm{cm}^{-3}$ & 1.317 & 1.318 \\
\hline $\mathrm{Z}$ & 24 & 24 \\
\hline $\mathrm{Mu}\left(\mathrm{mm}^{-1}\right)$ & 0.153 & 0.155 \\
\hline F000 & 8064.0 & 8064.0 \\
\hline F000' & 8070.79 & \\
\hline $\mathrm{h}, \mathrm{k}, \mathrm{l}_{\max }$ & $50,50,19$ & $50,50,19$ \\
\hline Nref & 29456 [15298] & 29386 \\
\hline $\mathrm{T}_{\min }, \mathrm{T}_{\max }$ & $0.938,0.963$ & $0.707,0.746$ \\
\hline $\mathrm{T}_{\min }$ & 0.938 & \\
\hline
\end{tabular}

Correction method $=\#$ Reported T Limits: $\mathrm{T}_{\min }=0.707 \mathrm{~T}_{\max }=0.746$

AbsCorr $=$ MULTI-SCAN

Data completeness $=1.92 / 1.00 \quad \operatorname{Theta}(\max )=27.564$

$\mathrm{R}($ reflections $)=0.0542(23768) \quad \mathrm{wR} 2($ reflections $)=0.1020(29386)$

$\mathrm{S}=1.121 \quad \mathrm{Npar}=1664$ 


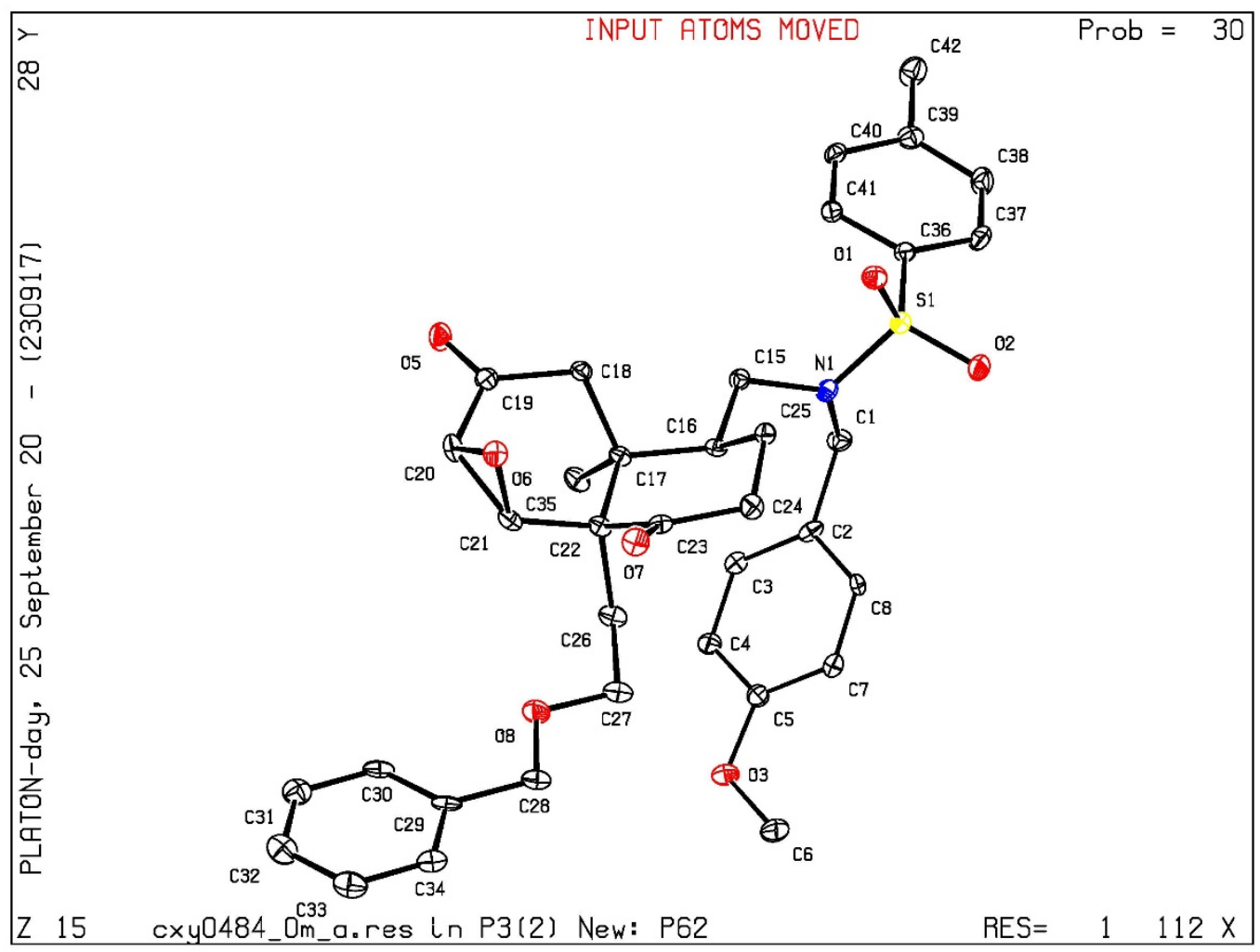


Crystal Data for Compound $29\left(\mathrm{C}_{28} \mathrm{H}_{41} \mathrm{NO}_{4} \mathrm{~S}\right)$ :

\begin{tabular}{|c|c|c|}
\hline \multirow{2}{*}{$\begin{array}{l}\text { Bond precision: } \\
\text { Cell: }\end{array}$} & \multirow{2}{*}{$\begin{array}{l}C-C=0.0041 \mathrm{~A} \\
a=9.098(6)\end{array}$} & Wavelength $=0.71073$ \\
\hline & & $c=13.365(6)$ \\
\hline \multirow{3}{*}{ Temperature: } & alpha $=90$ & gamma $=90$ \\
\hline & $100 \mathrm{~K}$ & \\
\hline & Calculated & Reported \\
\hline Volume & $1261.9(11)$ & $1261.9(10)$ \\
\hline Space group & P 21 & P 1211 \\
\hline Hall group & $\mathrm{P} 2 \mathrm{yb}$ & P 2yb \\
\hline Moiety formula & $\mathrm{C}_{28} \mathrm{H}_{41} \mathrm{~N} \mathrm{O}_{4} \mathrm{~S}$ & $\mathrm{C}_{28} \mathrm{H}_{41} \mathrm{~N} \mathrm{O}_{4} \mathrm{~S}$ \\
\hline Sum formula & $\mathrm{C}_{28} \mathrm{H}_{41} \mathrm{~N} \mathrm{O}_{4} \mathrm{~S}$ & $\mathrm{C}_{28} \mathrm{H}_{41} \mathrm{~N} \mathrm{O}_{4} \mathrm{~S}$ \\
\hline $\mathrm{Mr}$ & 487.68 & 487.68 \\
\hline $\mathrm{Dx}, \mathrm{g} \mathrm{cm}^{-3}$ & 1.283 & 1.284 \\
\hline $\mathrm{Z}$ & 2 & 2 \\
\hline $\mathrm{Mu}\left(\mathrm{mm}^{-1}\right)$ & 0.163 & 0.163 \\
\hline F000 & 528.0 & 528.0 \\
\hline F000' & 528.47 & \\
\hline $\mathrm{h}, \mathrm{k}, \mathrm{l}_{\max }$ & $11,13,17$ & $11,13,17$ \\
\hline Nref & $5781[3046]$ & 5742 \\
\hline $\mathrm{T}_{\min }, \mathrm{T}_{\max }$ & $0.929,0.945$ & $0.544,0.746$ \\
\hline$T_{\min }$ & 0.929 & \\
\hline
\end{tabular}

Correction method $=\#$ Reported T Limits: $\mathrm{T}_{\min }=0.544 \mathrm{~T}_{\max }=0.746$

AbsCorr $=$ MULTI - SCAN

Data completeness $=1.89 / 0.99 \quad \operatorname{Theta}(\max )=27.496$

$\mathrm{R}($ reflections $)=0.0474(5430) \quad \mathrm{wR} 2($ reflections $)=0.1216(5742)$

$\mathrm{S}=1.044 \quad \mathrm{Npar}=313$ 


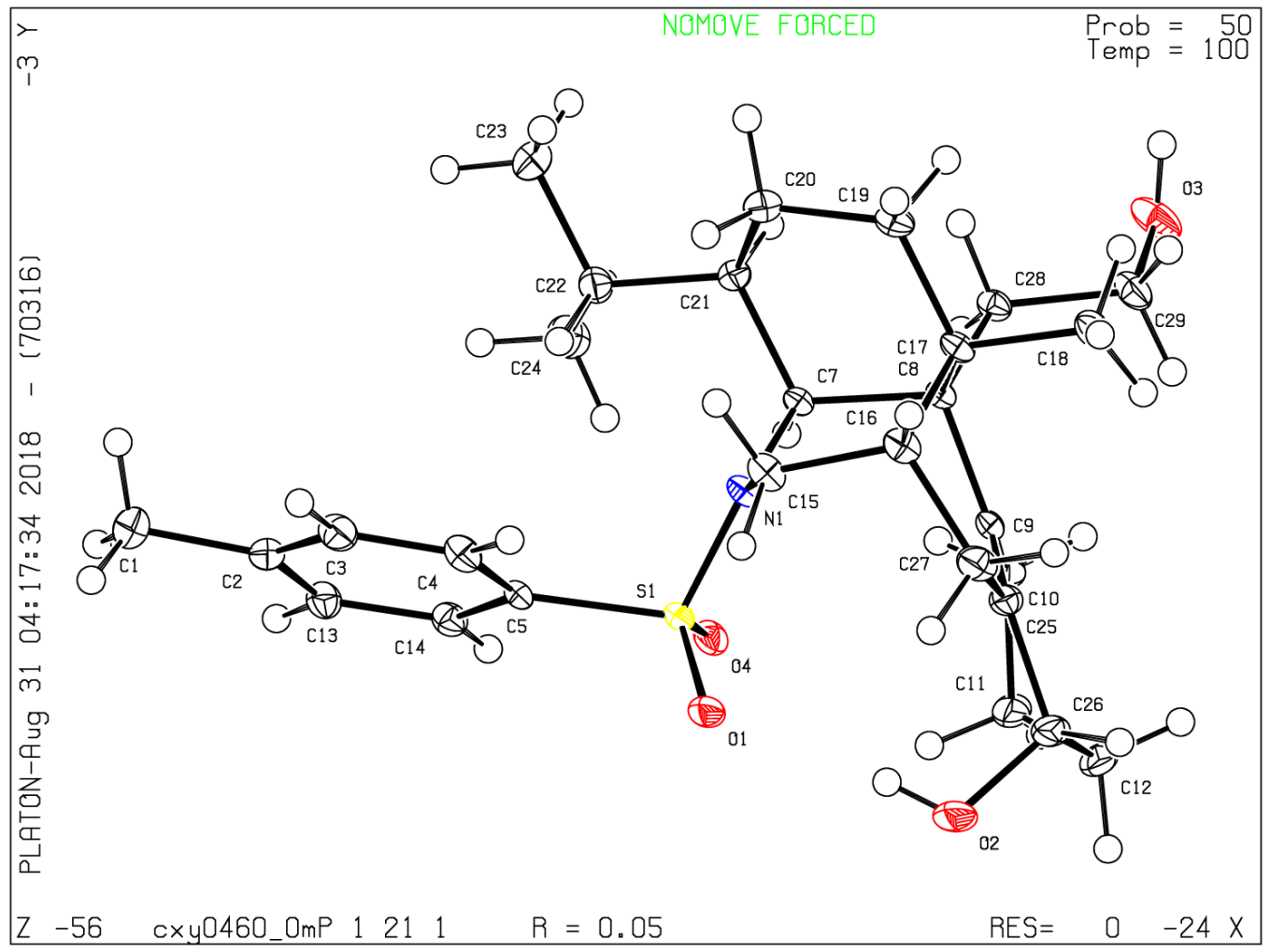


Crystal Data for Compound $\mathbf{3 8}\left(\mathrm{C}_{21} \mathrm{H}_{33} \mathrm{NO}_{2}\right)$ :

\begin{tabular}{|c|c|c|}
\hline Bond precision: & $\mathrm{C}-\mathrm{C}=0.0033 \mathrm{~A}$ & Wavelength $=1.54178$ \\
\hline Cell: & $\mathrm{a}=8.2356(10)$ & $b=14.7564(17)$ \\
\hline \multirow{3}{*}{ Temperature: } & alpha $=90$ & gamma $=90$ \\
\hline & $100 \mathrm{~K}$ & \\
\hline & Calculated & Reported \\
\hline Volume & $1793.3(3)$ & 1793.3(3) \\
\hline Space group & P 212121 & P 212121 \\
\hline Hall group & P $2 a c 2 a b$ & P $2 a c 2 a b$ \\
\hline Moiety formula & $\mathrm{C}_{21} \mathrm{H}_{33} \mathrm{~N} \mathrm{O}_{2}$ & $\mathrm{C}_{21} \mathrm{H}_{33} \mathrm{~N} \mathrm{O}_{2}$ \\
\hline Sum formula & $\mathrm{C}_{21} \mathrm{H}_{33} \mathrm{~N} \mathrm{O}_{2}$ & $\mathrm{C}_{21} \mathrm{H}_{34} \mathrm{~N} \mathrm{O}_{2}$ \\
\hline $\mathrm{Mr}$ & 331.48 & 332.49 \\
\hline $\mathrm{Dx}, \mathrm{g} \mathrm{cm}^{-3}$ & 1.228 & 1.231 \\
\hline $\mathrm{Z}$ & 4 & 4 \\
\hline $\mathrm{Mu}\left(\mathrm{mm}^{-1}\right)$ & 0.600 & 0.600 \\
\hline F000 & 728.0 & 732.0 \\
\hline F000' & 729.94 & \\
\hline $\mathrm{h}, \mathrm{k}, \mathrm{l}_{\max }$ & $10,18,18$ & $9,18,18$ \\
\hline Nref & 3846 [ 2203] & 3687 \\
\hline $\mathrm{T}_{\min }, \mathrm{T}_{\max }$ & $0.917,0.942$ & $0.649,0.754$ \\
\hline $\mathrm{T}_{\min }$ & 0.914 & \\
\hline
\end{tabular}

Correction method $=\#$ Reported T Limits: $\mathrm{T}_{\min }=0.649 \mathrm{~T}_{\max }=0.754$

AbsCorr $=$ MULTI - SCAN

Data completeness $=1.67 / 0.96 \quad \operatorname{Theta}(\max )=77.959$

$\mathrm{R}($ reflections $)=0.0420(3555) \quad \mathrm{wR} 2($ reflections $)=0.1180(3687)$

$\mathrm{S}=1.062 \quad \mathrm{Npar}=221$ 


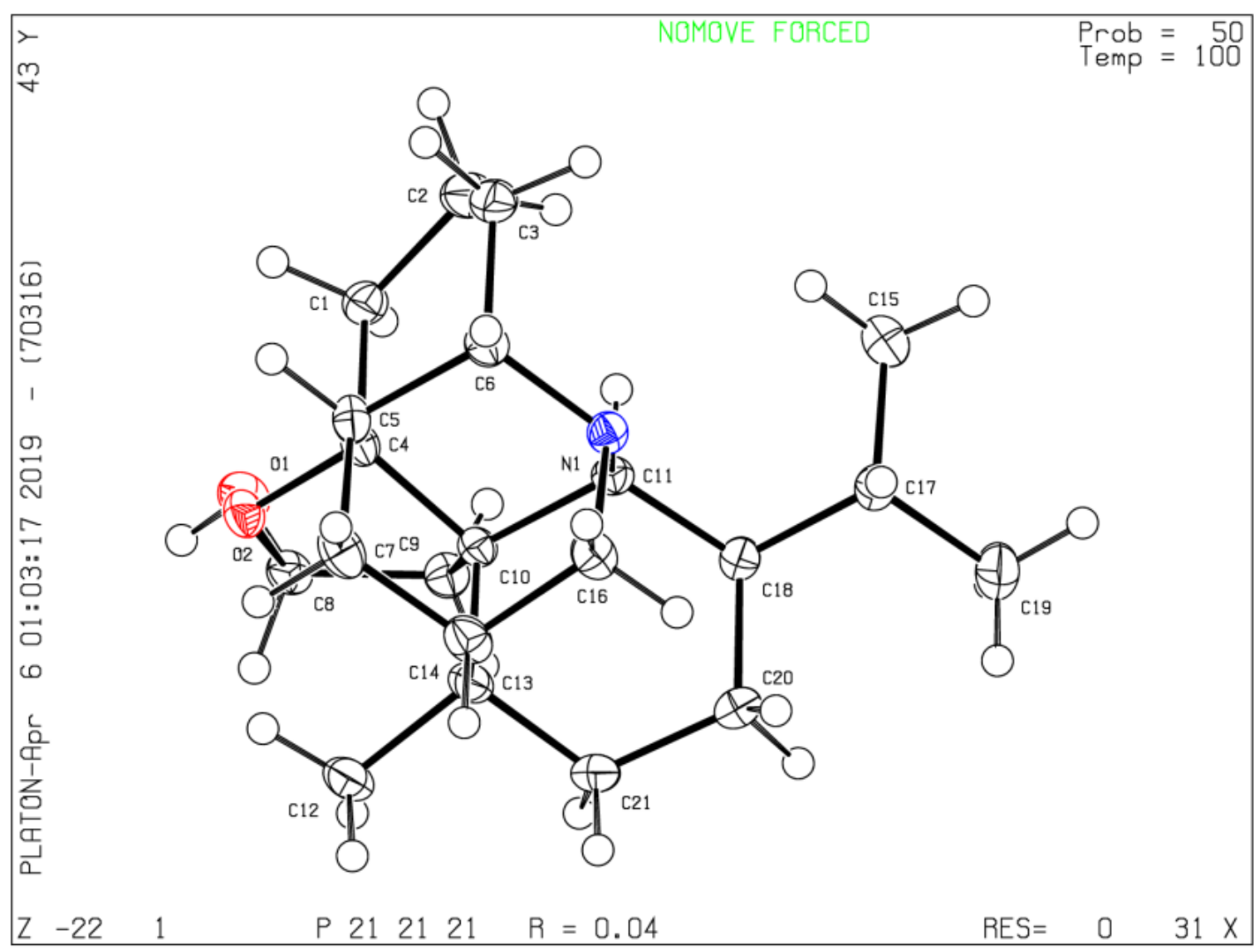




\section{Reference}

1. Ramachary, D. B.; Kishor, M. Organocatalytic Sequential One-Pot Double Cascade AsymmetricSynthesis of Wieland-Miescher Ketone Analogues from a Knoevenagel/ Hydrogenation/Robinson Annulation Sequence: Scopeand Applications of Organocatalytic Biomimetic Reductions. J. Org. Chem. 2007, 72, $5056-5068$.

2. Lechner, R.; König, B. Oxidation and Deprotection of Primary Benzylamines by Visible Light Flavin Photocatalysis. Synthesis 2010, 10, $1712-1718$.

3. Mukaiyama, T.; Matsuo, J.; Yanagisawa, M. A New and Efficient Method for Oxidation of Various Alcohols by Using N-tert-Butyl Phenylsulfinimidoyl Chloride. Chem. Lett 2000, 29, $1072-1073$.

4. Zhang, Y.; Di, Y.-T.; Mu, S.-Z.; Li, C.-S.; Zhang, Q.; Tan, C.-J.; Zhang, Z.; Fang, X.; Hao, X.-J. Dapholdhamines A-D, alkaloids from Daphniphyllum oldhami. J. Nat. Prod. 2009, 72, $1325-1327$ 
${ }^{1} \mathrm{H}$ NMR of 6

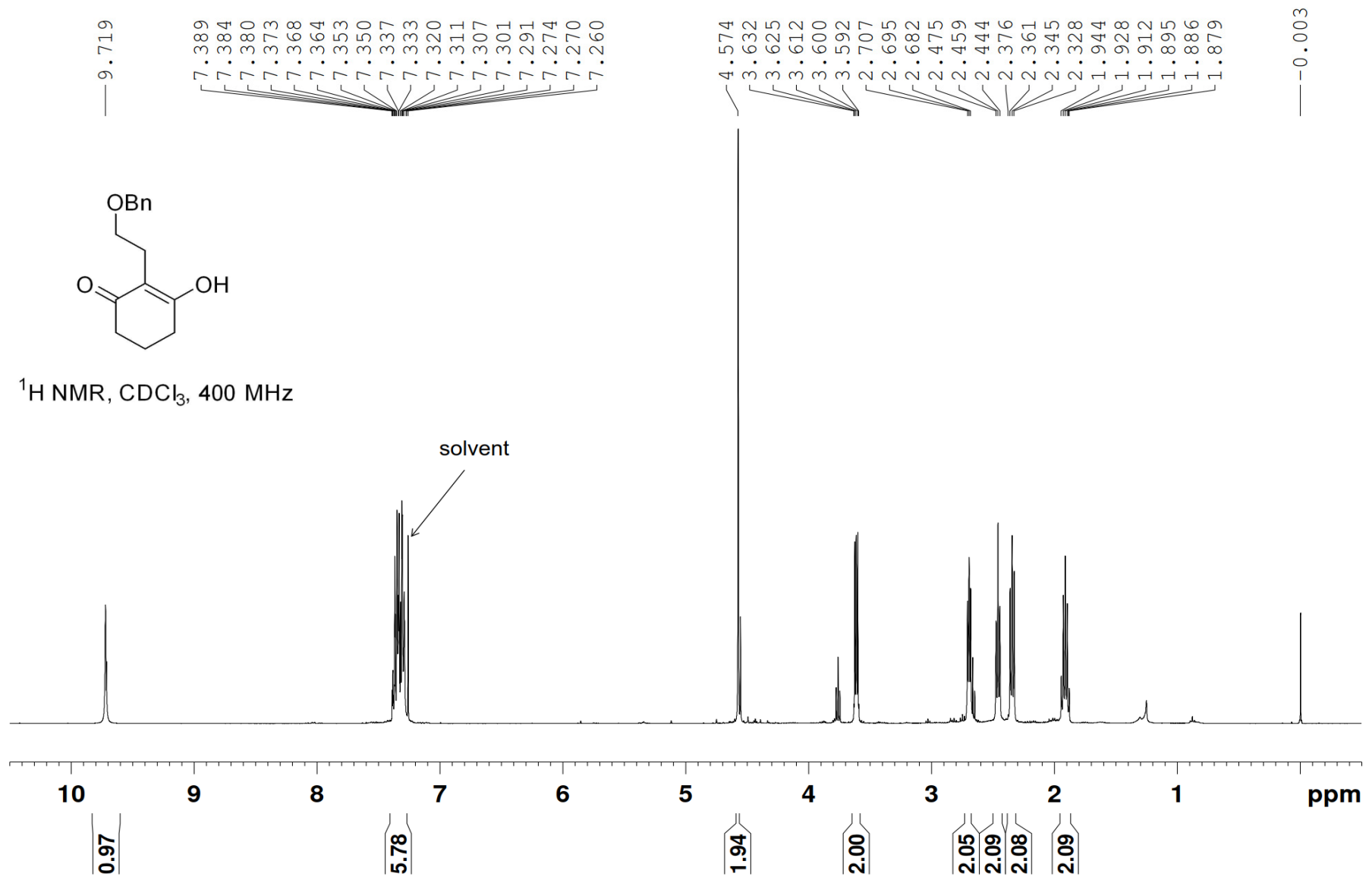


${ }^{13} \mathrm{C}$ NMR of 6

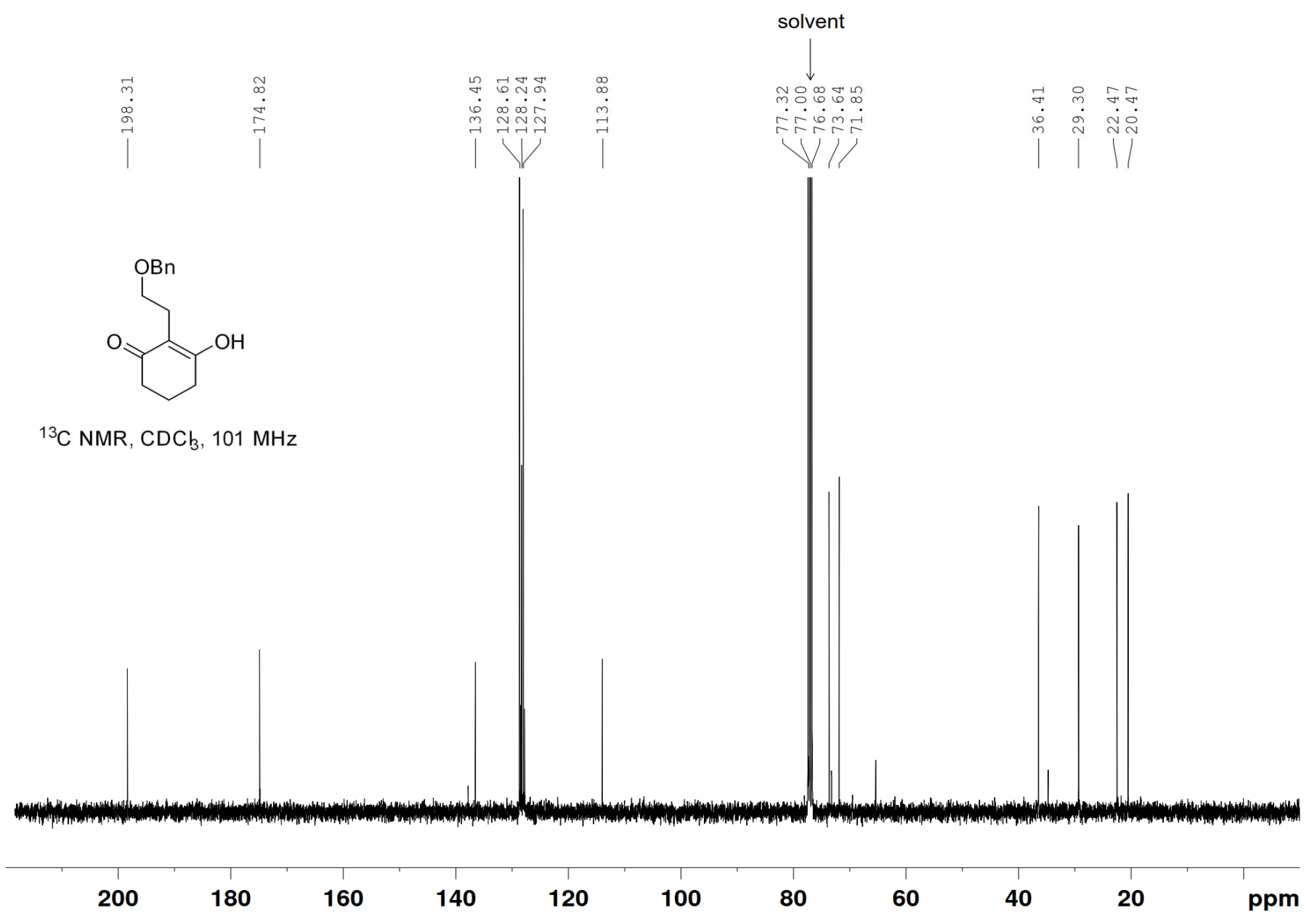




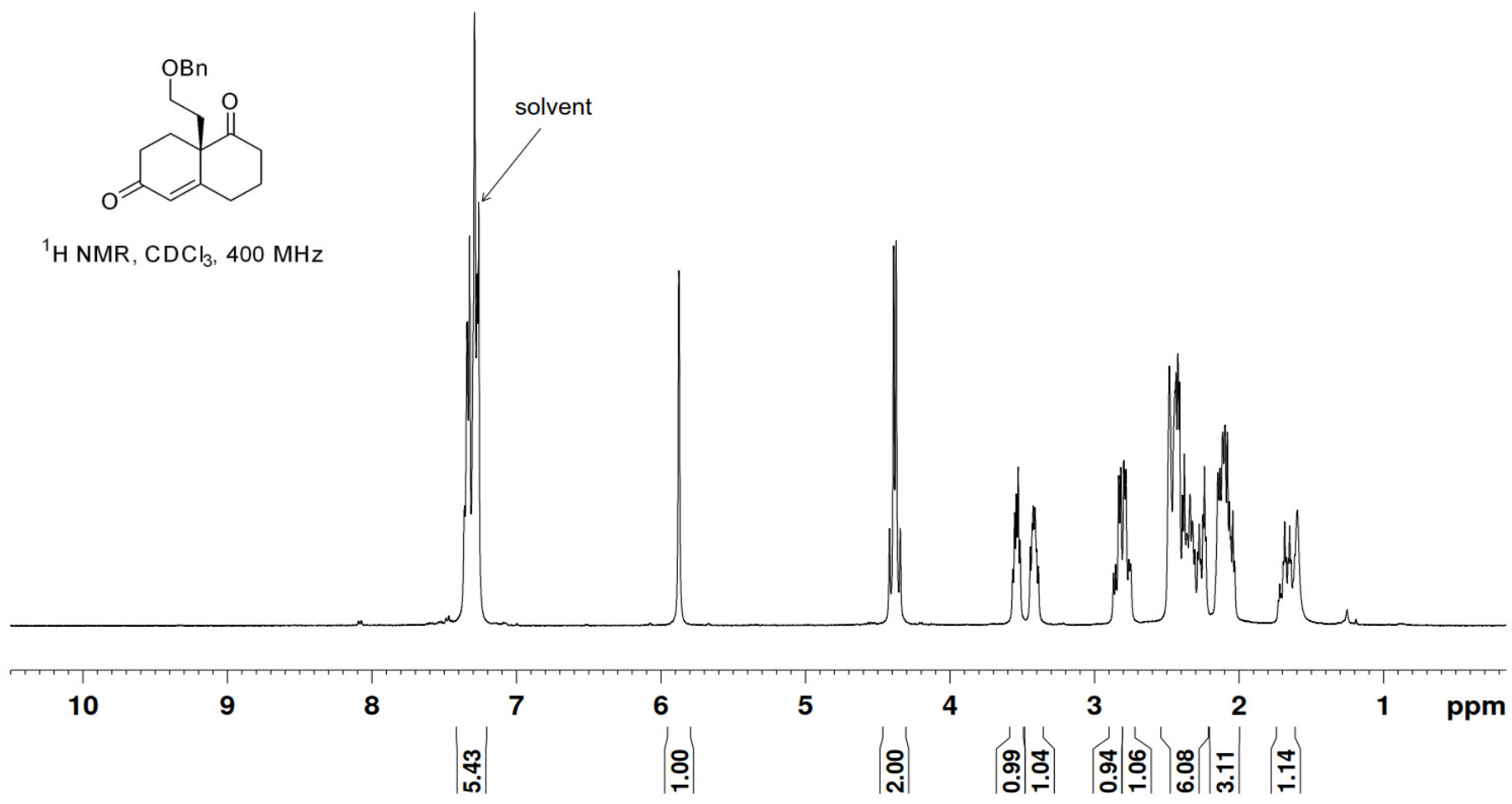


${ }^{13} \mathrm{C}$ NMR of 5

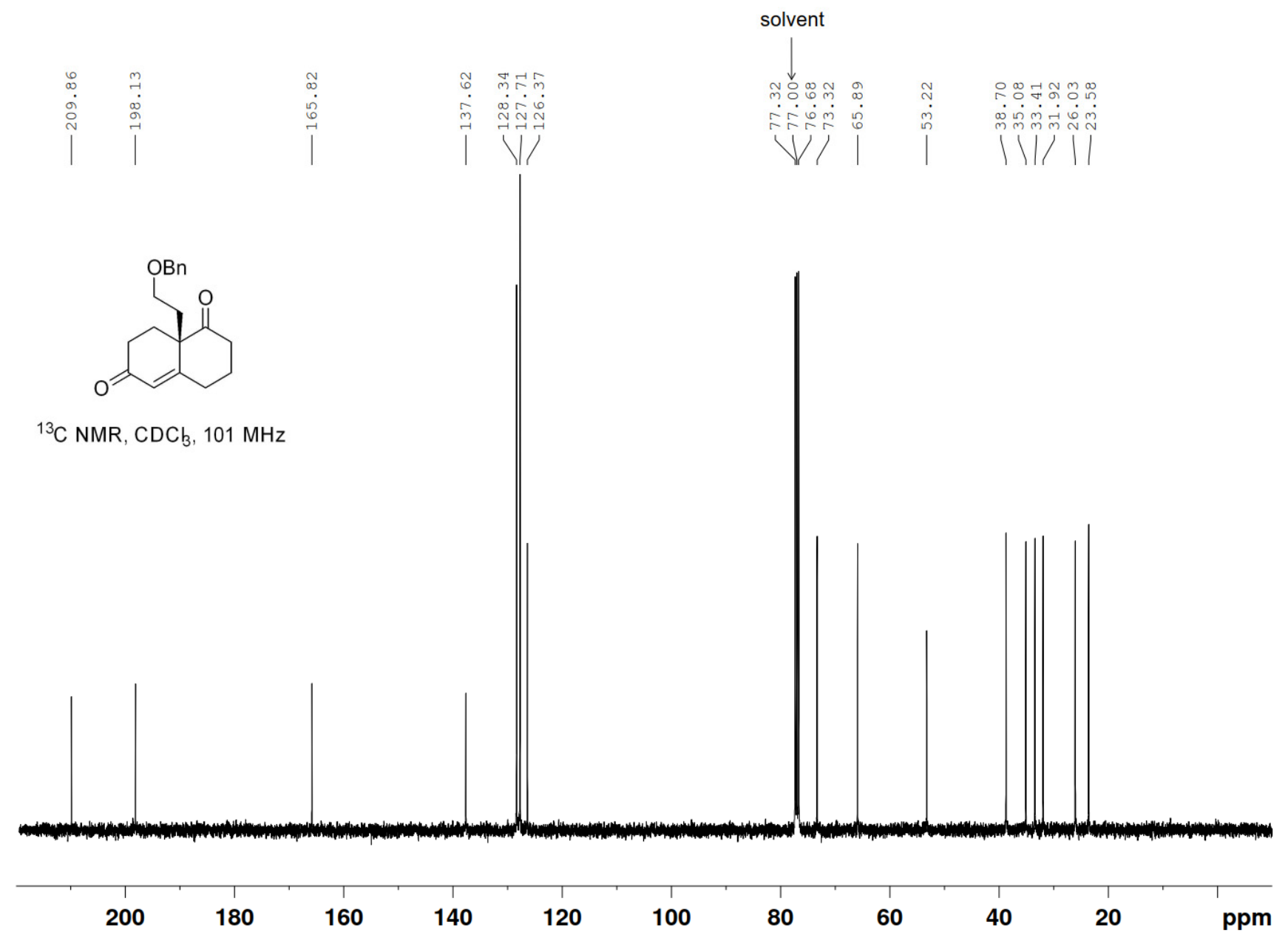




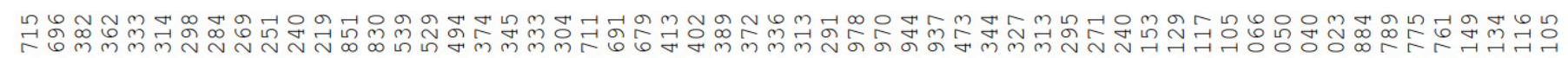

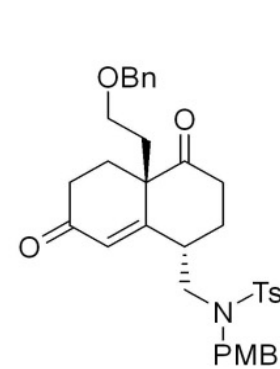

${ }^{1} \mathrm{H} \mathrm{NMR}, \mathrm{CDCl}_{3}, 400 \mathrm{MHz}$

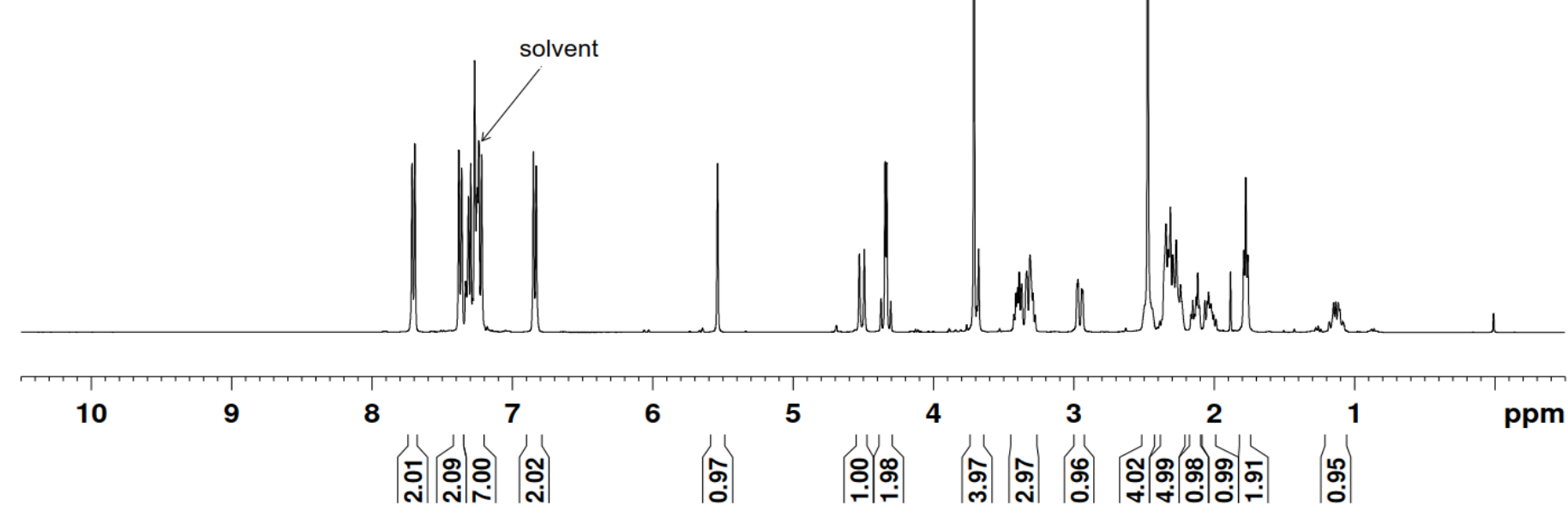


${ }^{13} \mathrm{C}$ NMR of 9

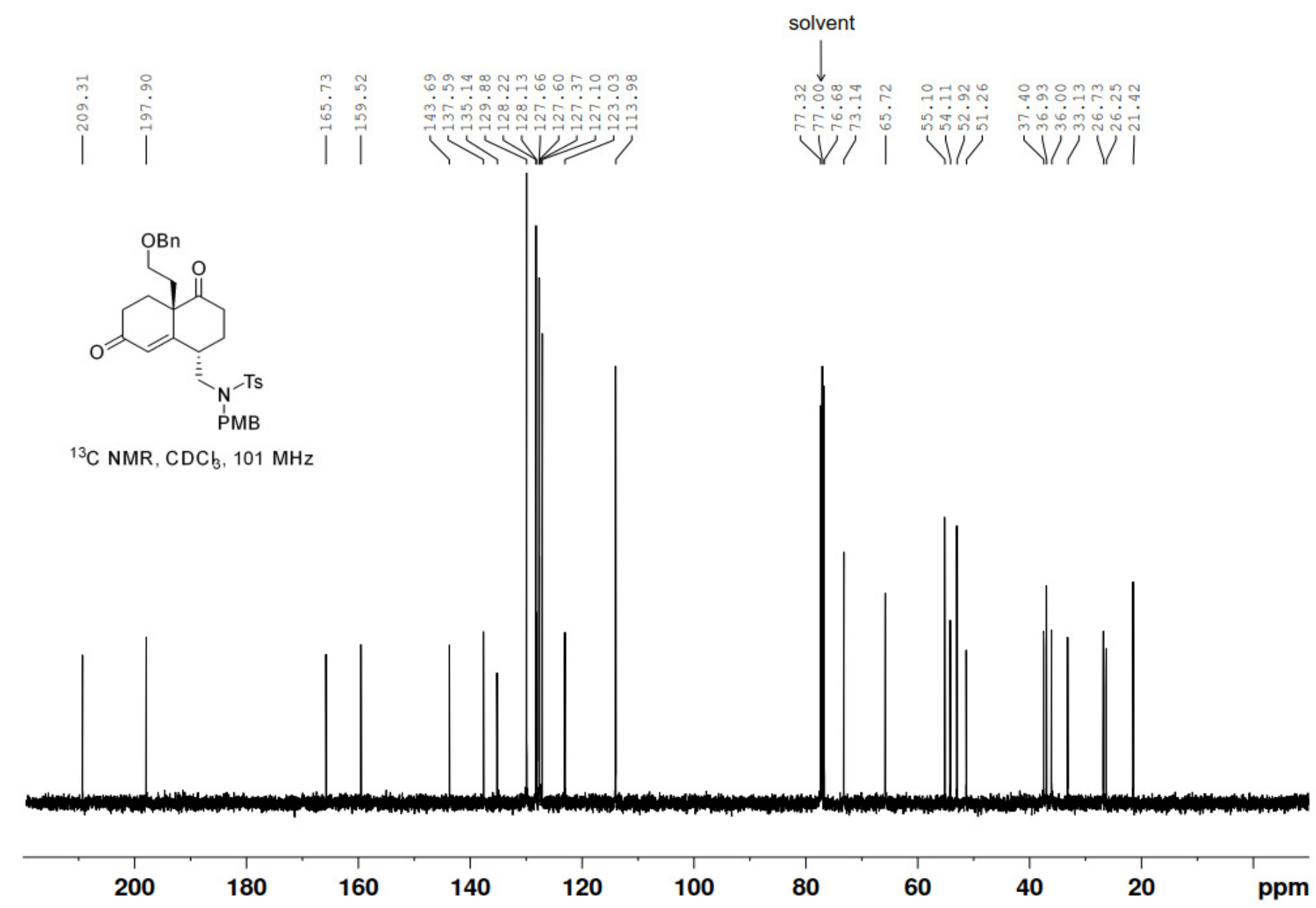




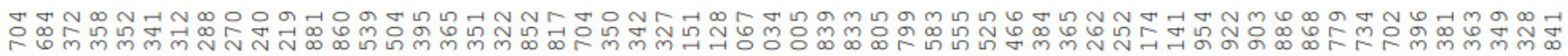

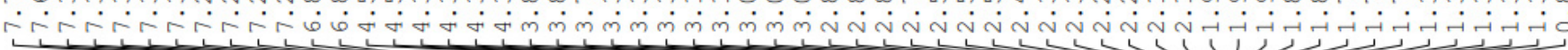

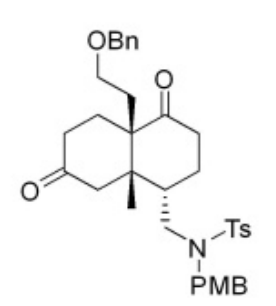

${ }^{1} \mathrm{HNMR}, \mathrm{CDCl}_{3}, 400 \mathrm{MHz}$

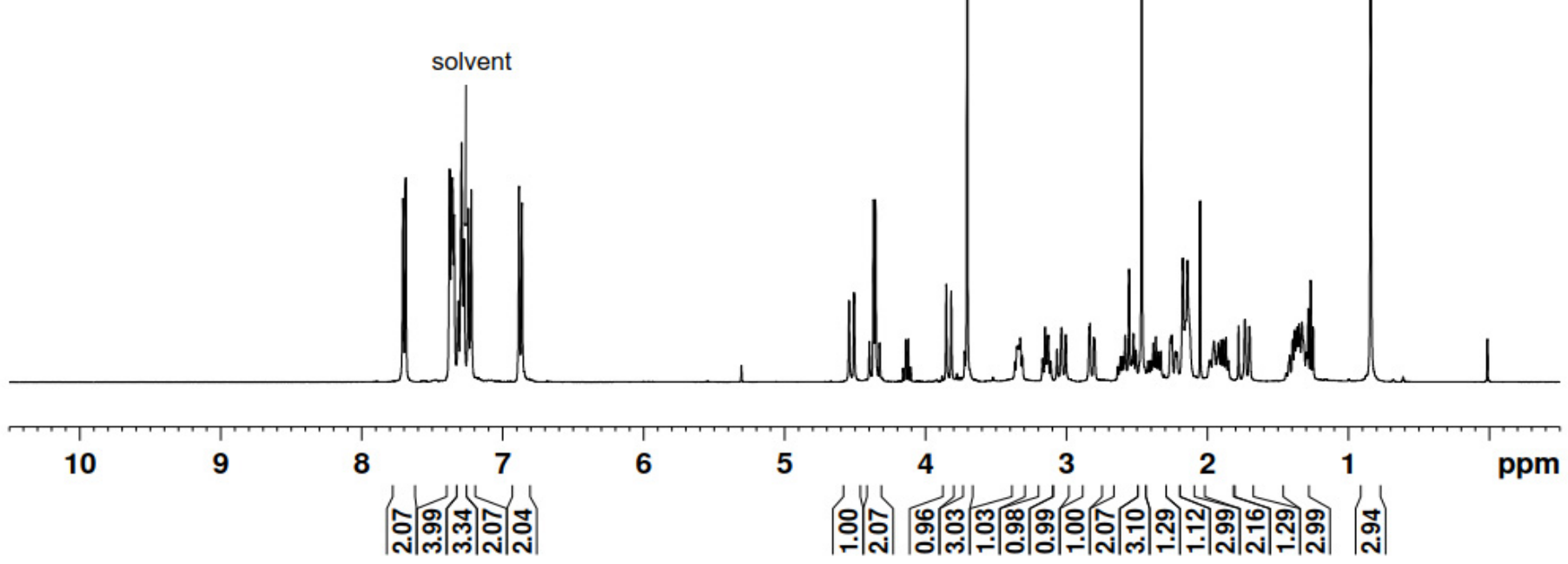


${ }^{13} \mathrm{C}$ NMR of $\mathbf{1 0}$

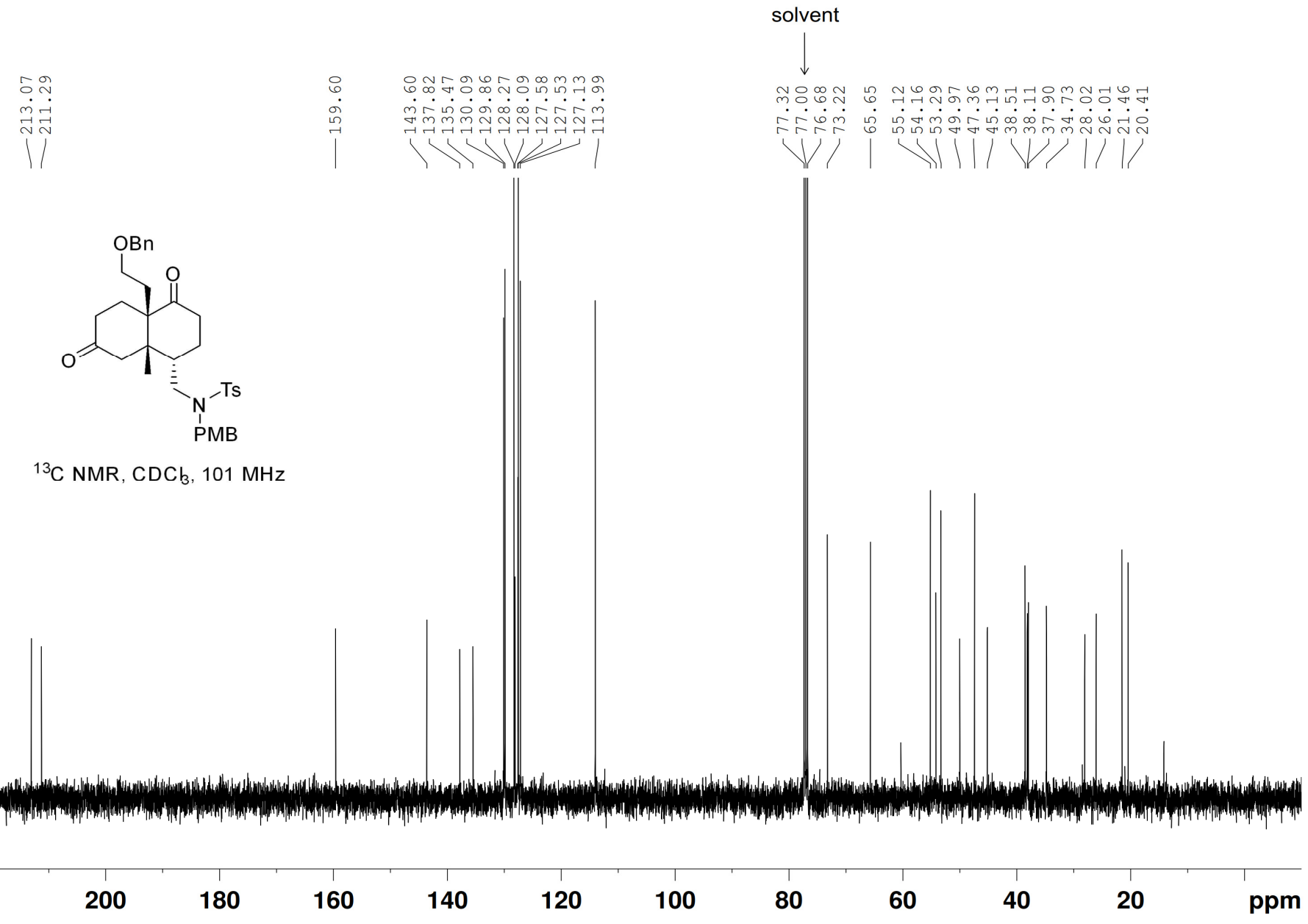




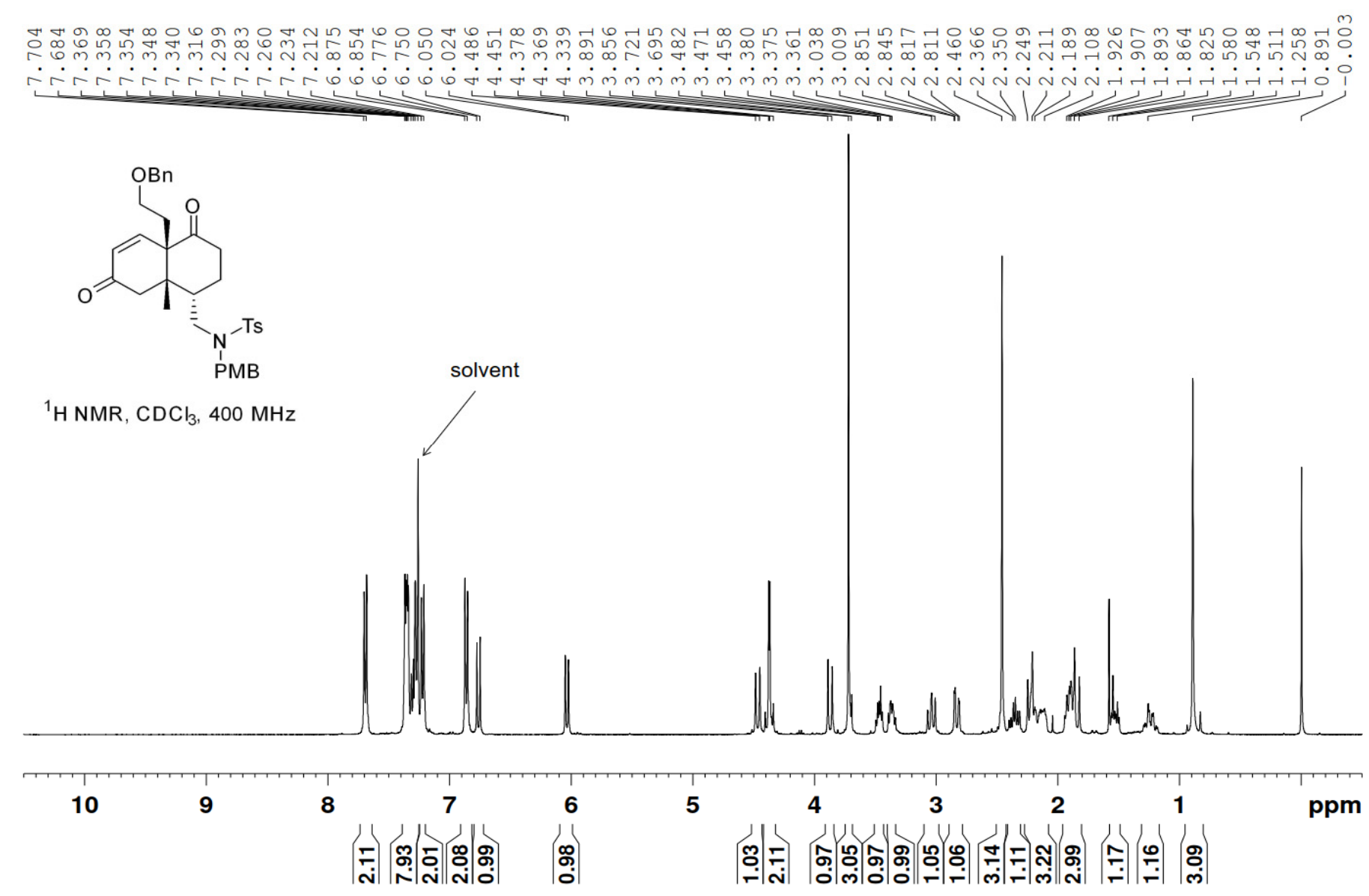


${ }^{13} \mathrm{C}$ NMR of $\mathbf{1 2}$

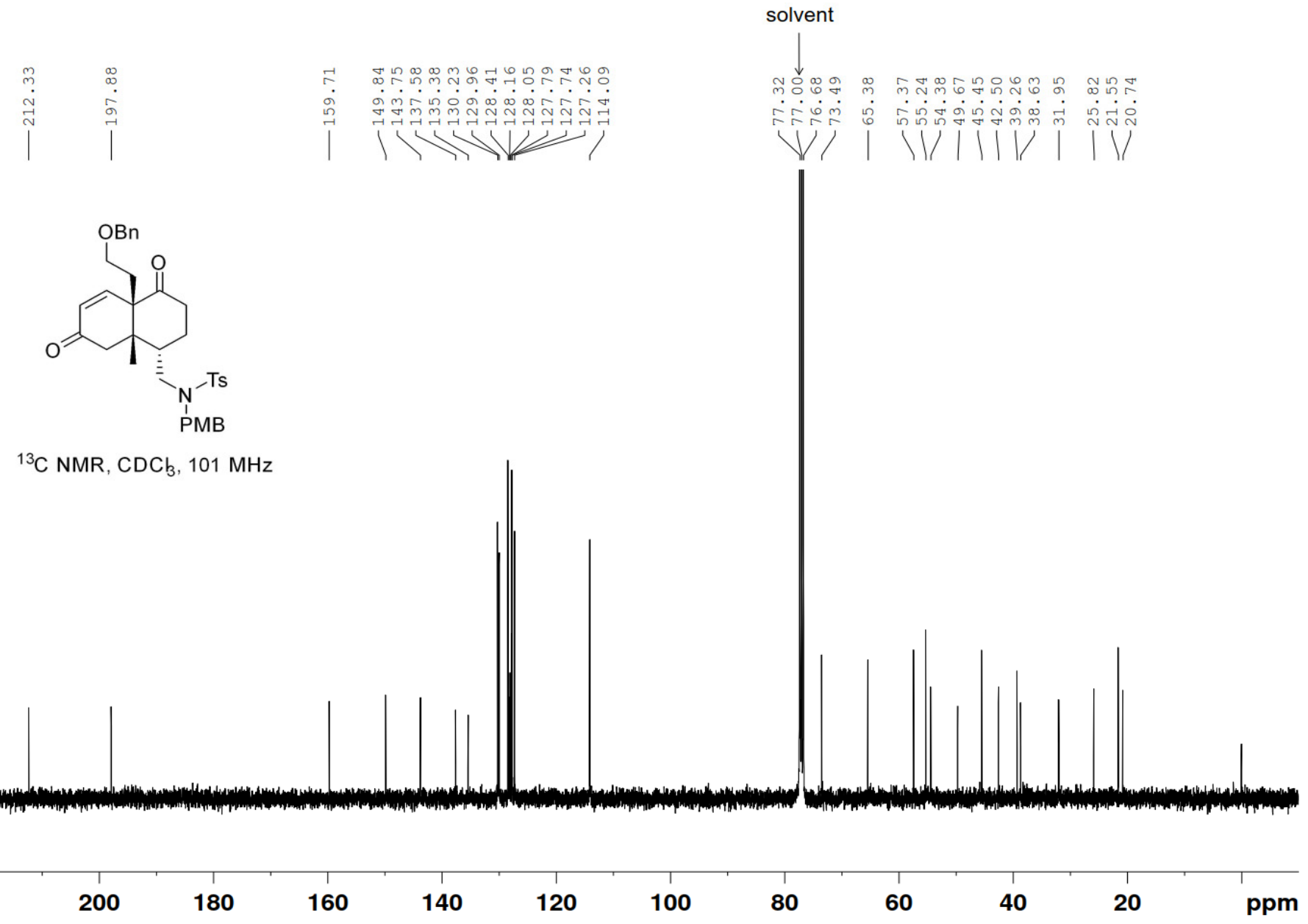


${ }^{1} \mathrm{H}$ NMR of $( \pm)-\mathbf{1 3}$

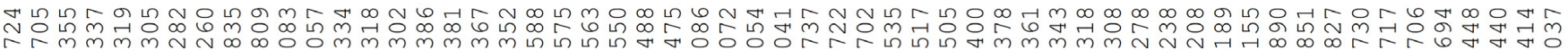

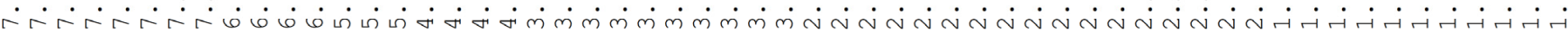

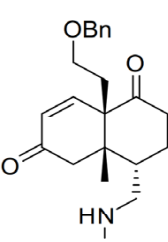

${ }^{1} \mathrm{H} \mathrm{NMR}, \mathrm{CDCl}_{3}, 400 \mathrm{MHz}$

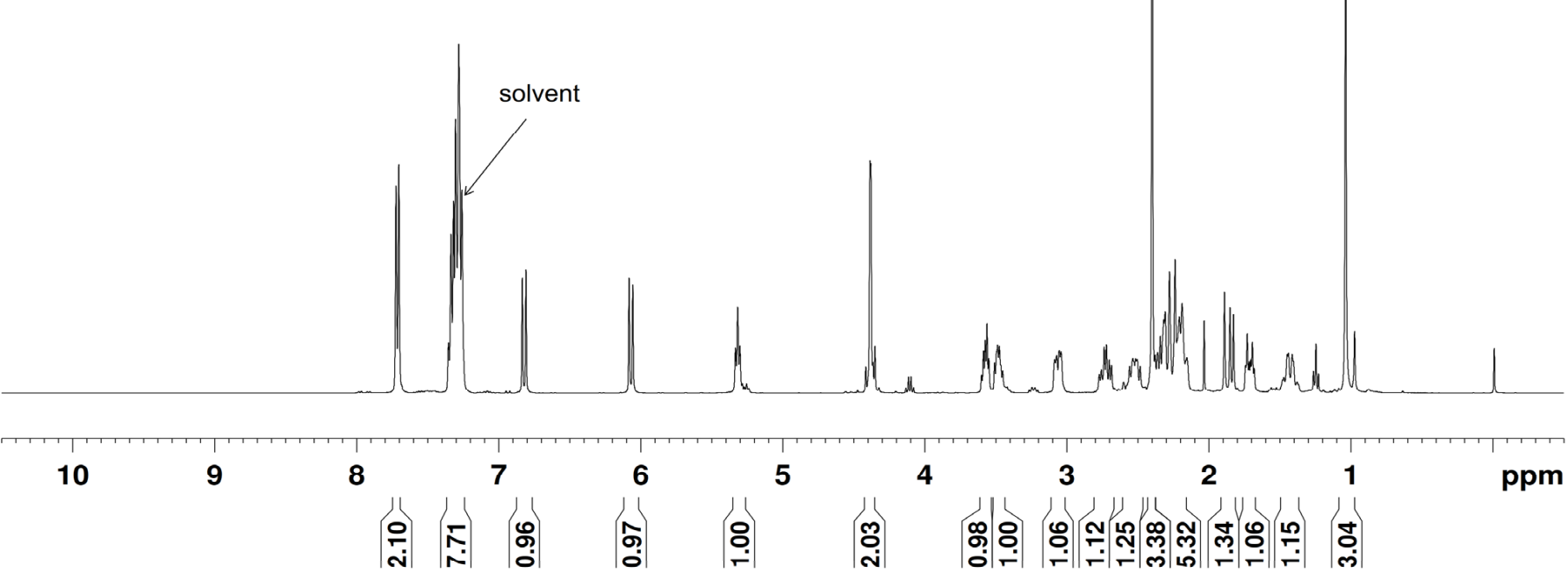


${ }^{13} \mathrm{C}$ NMR of $( \pm)-13$

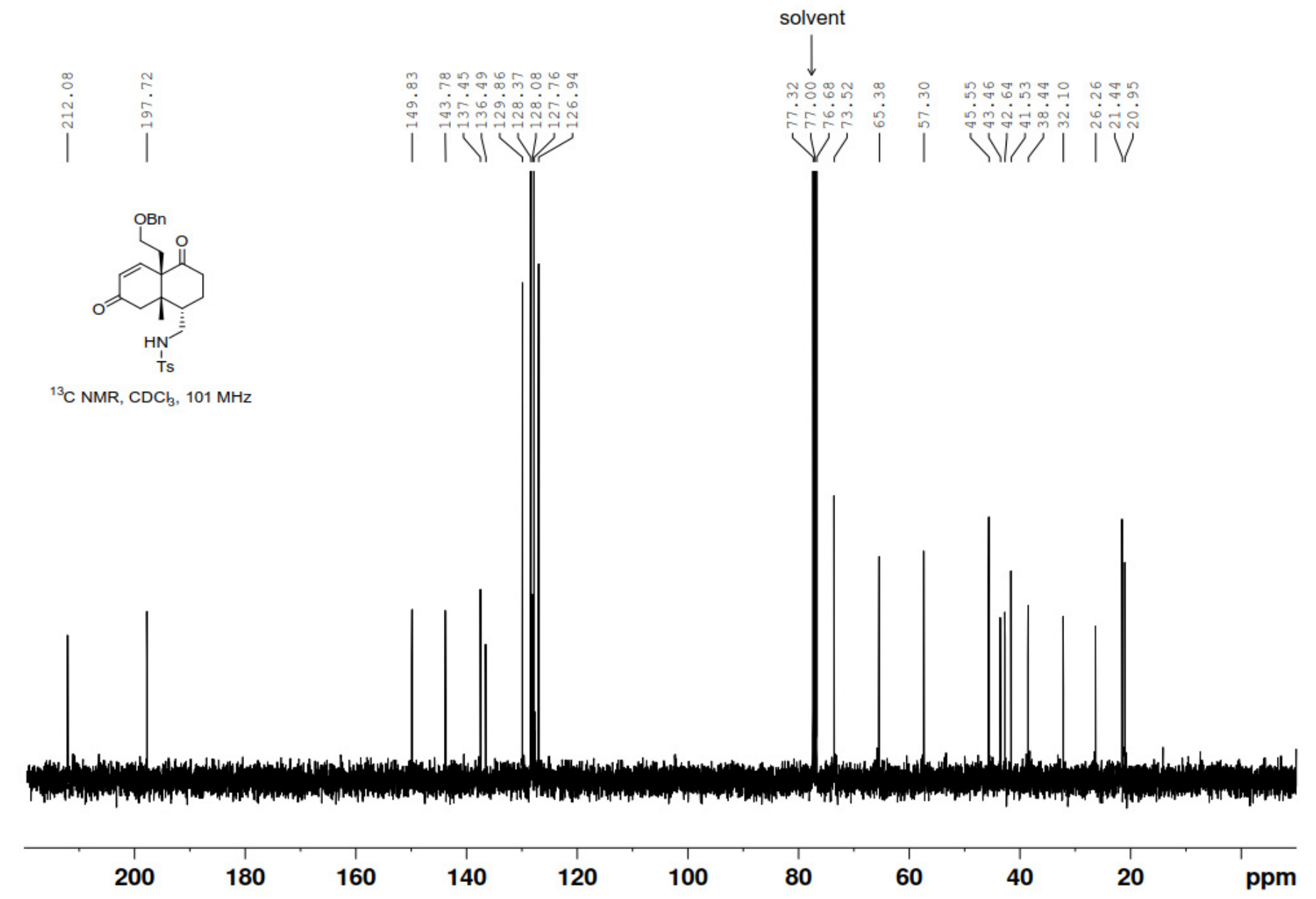


${ }^{1} \mathrm{H}$ NMR of $( \pm)-15$

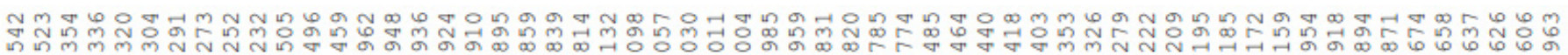

-

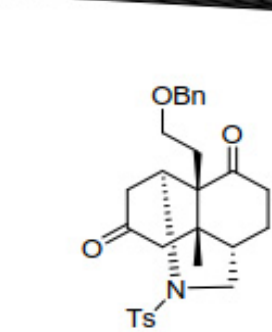

${ }^{1} \mathrm{H} \mathrm{NMR,} \mathrm{CDCl}_{3}, 400 \mathrm{MHz}$

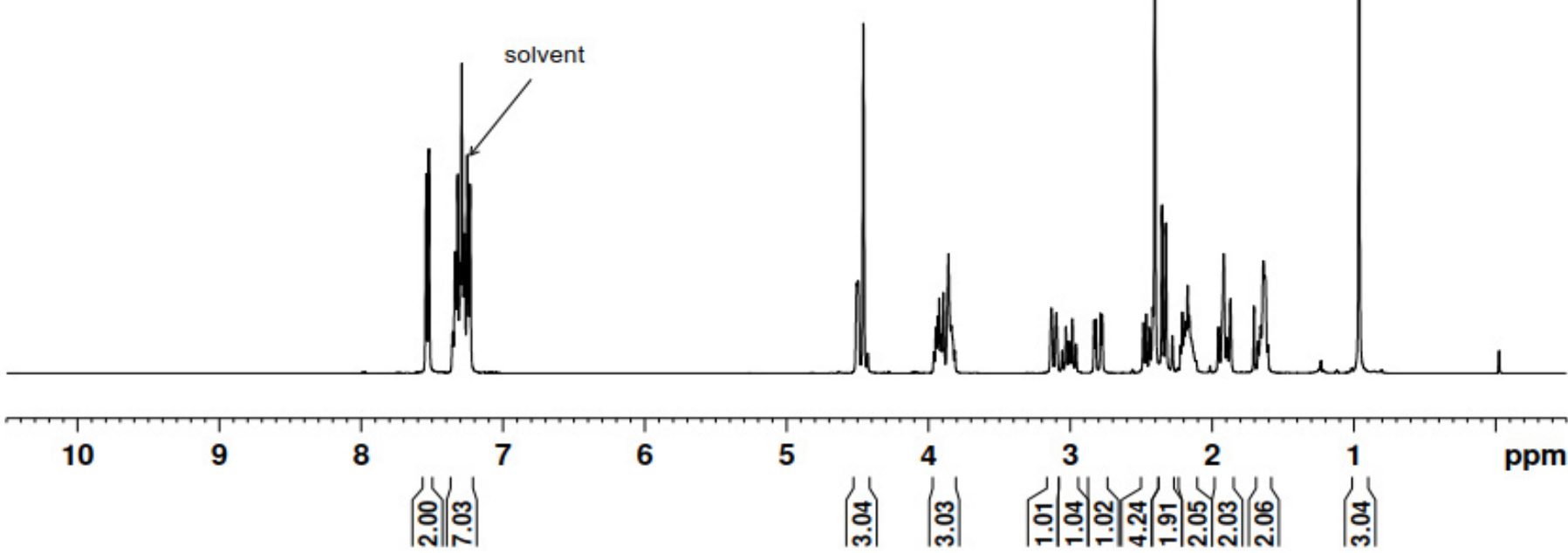


${ }^{13} \mathrm{C}$ NMR of $( \pm)-15$

|

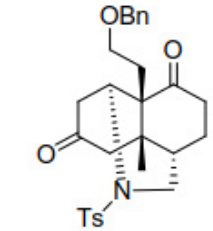

${ }^{13} \mathrm{C}$ NMR, $\mathrm{CDCl}_{3}, 101 \mathrm{MHz}$
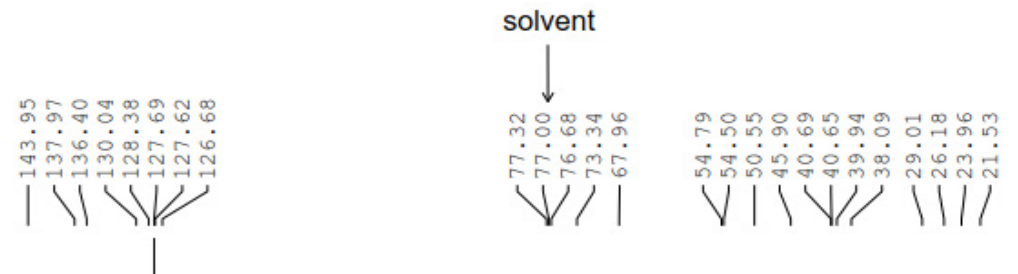

VI।

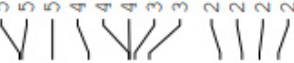

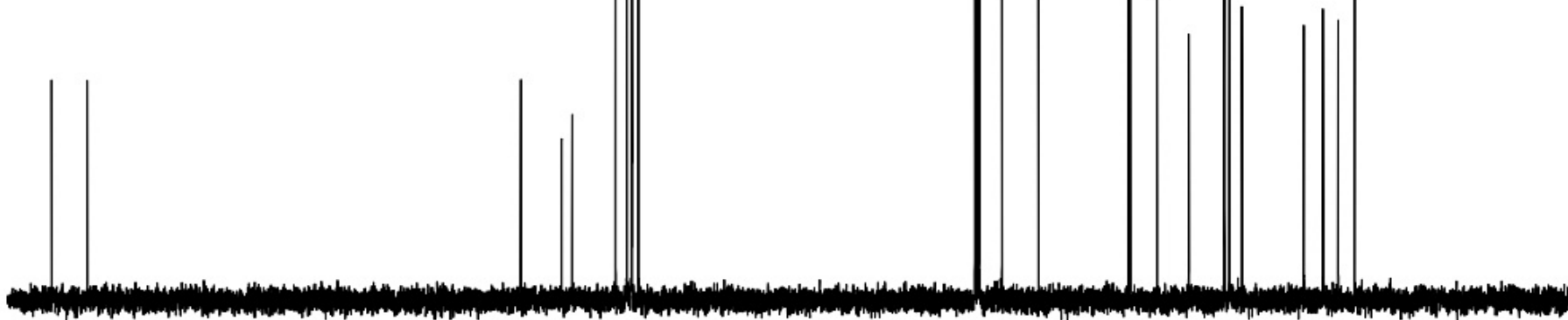

200 


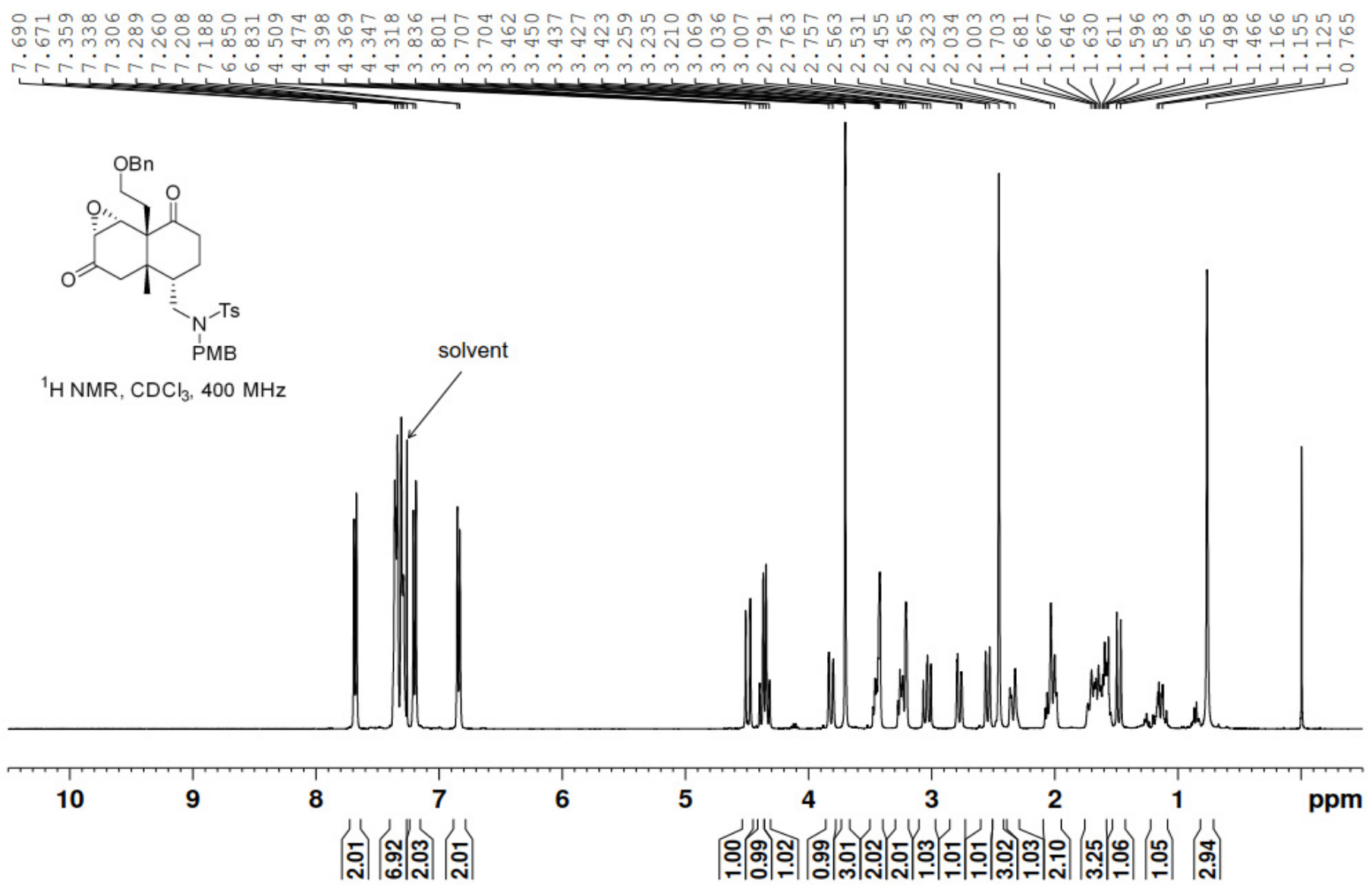


${ }^{13} \mathrm{C}$ NMR of 16

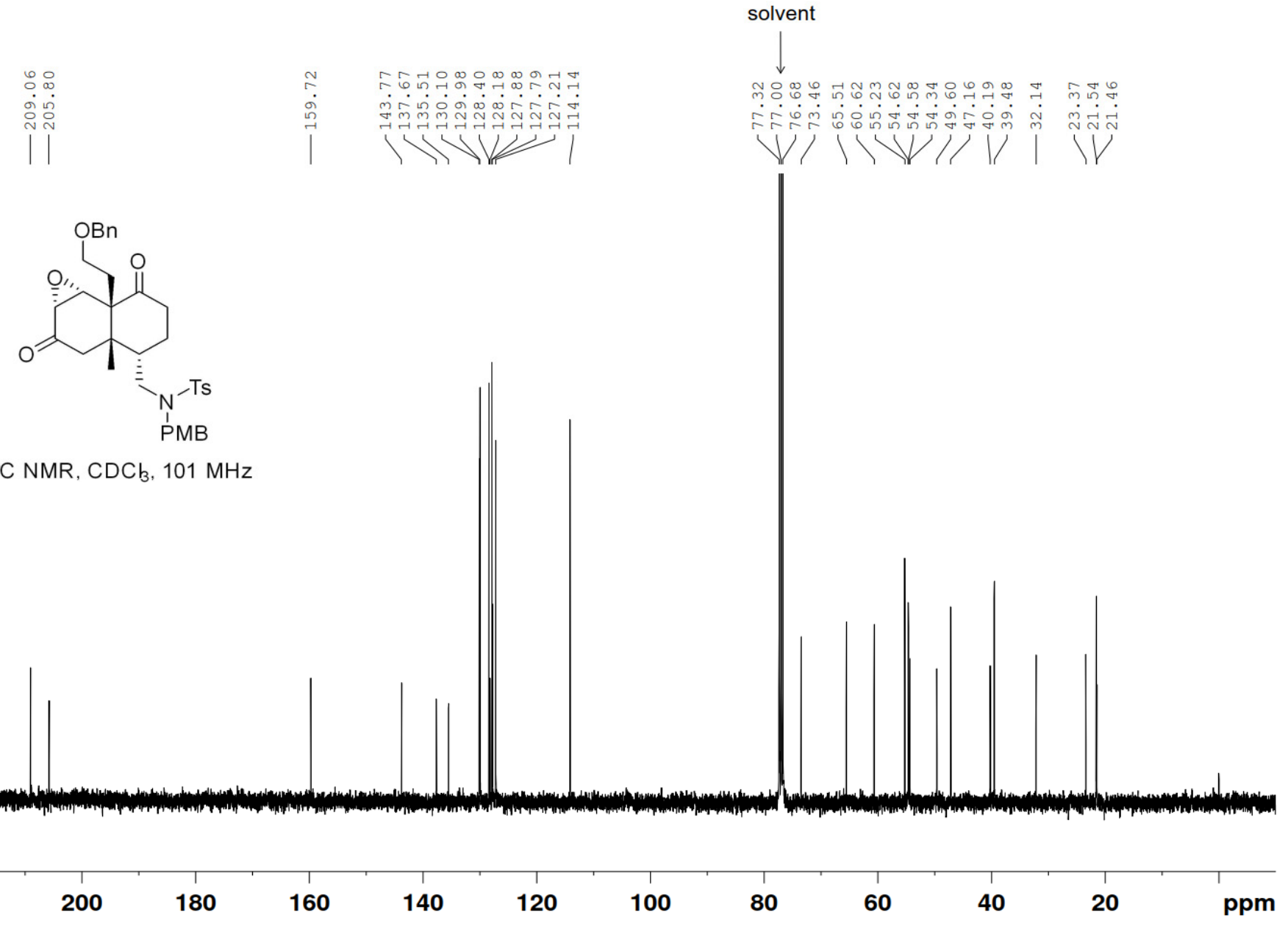




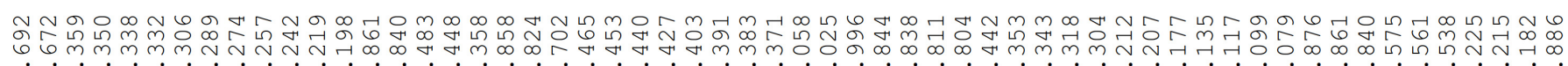

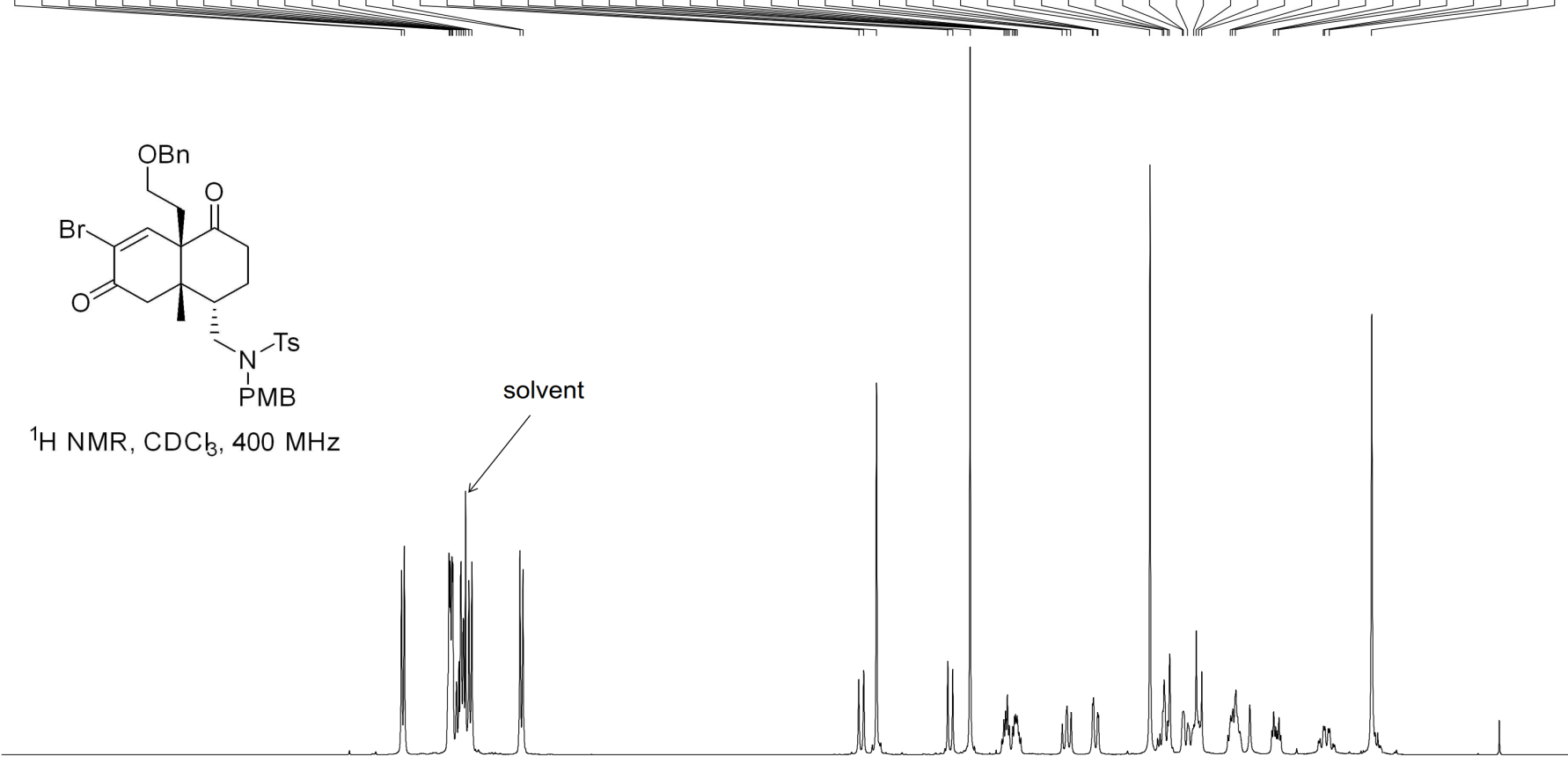

$10 \quad 9$


${ }^{13} \mathrm{C}$ NMR of $\mathbf{1 7}$

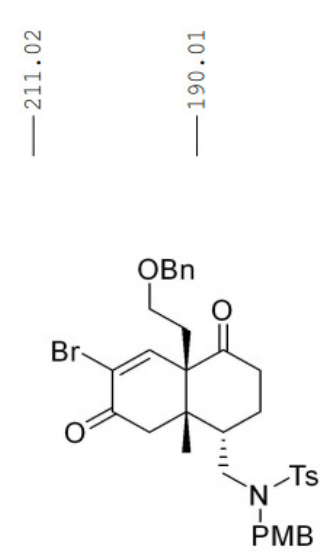

${ }^{13} \mathrm{C}$ NMR, $\mathrm{CDC}_{3}, 101 \mathrm{MHz}$

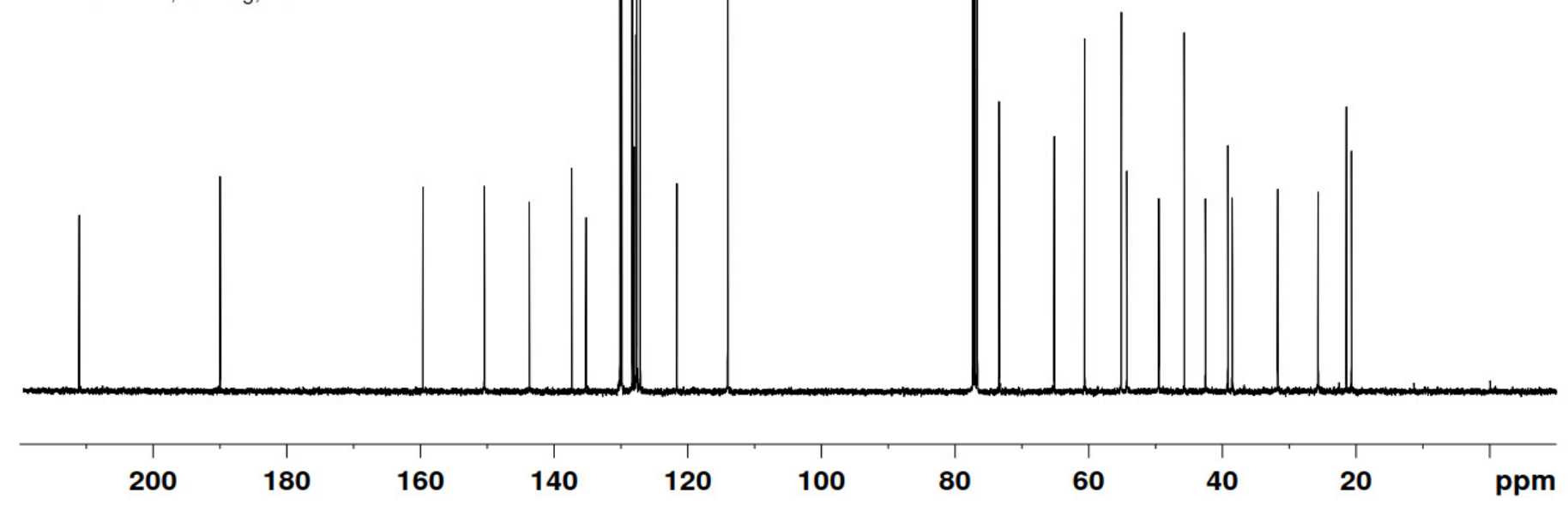




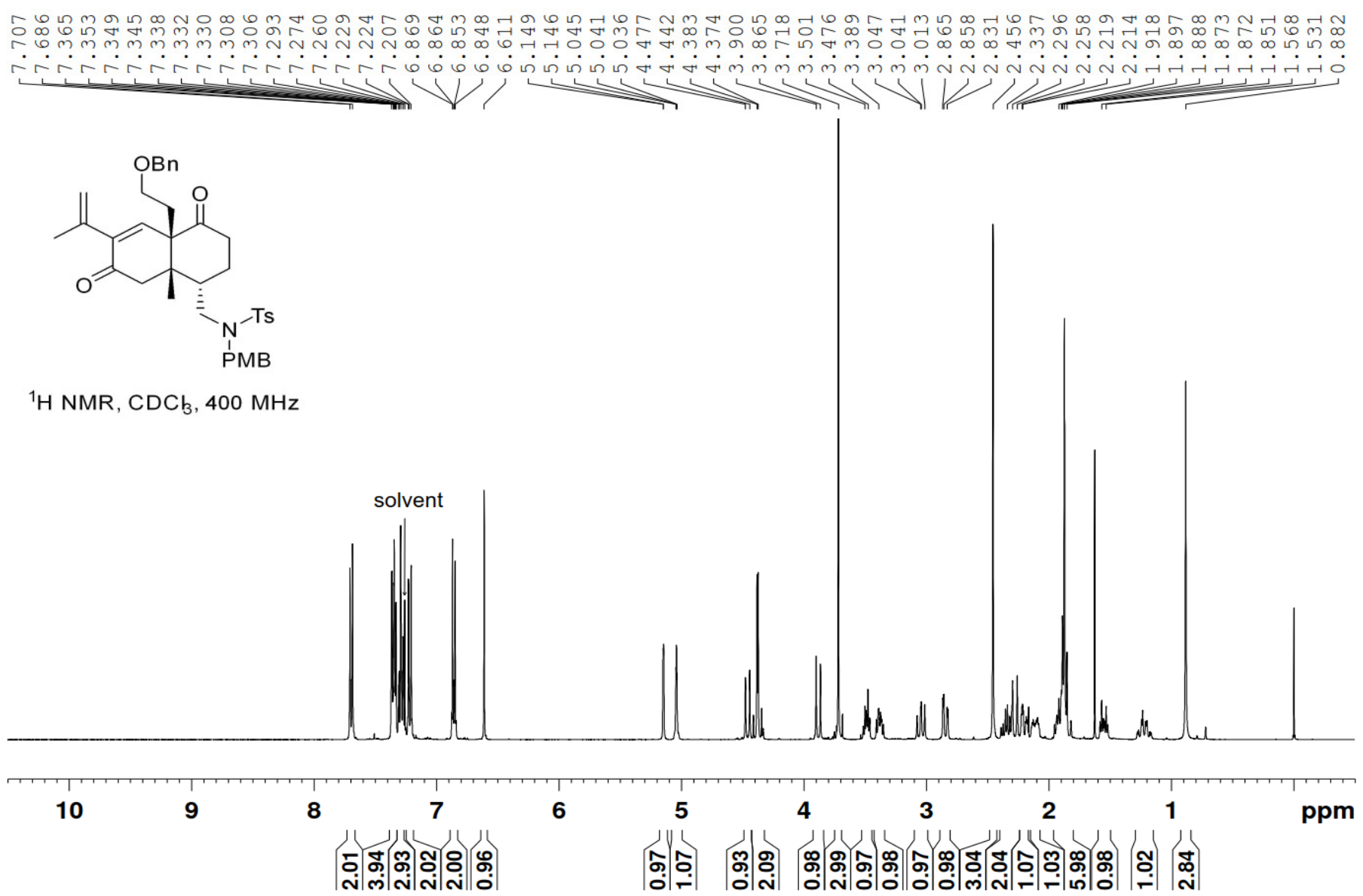


${ }^{13} \mathrm{C}$ NMR of 19

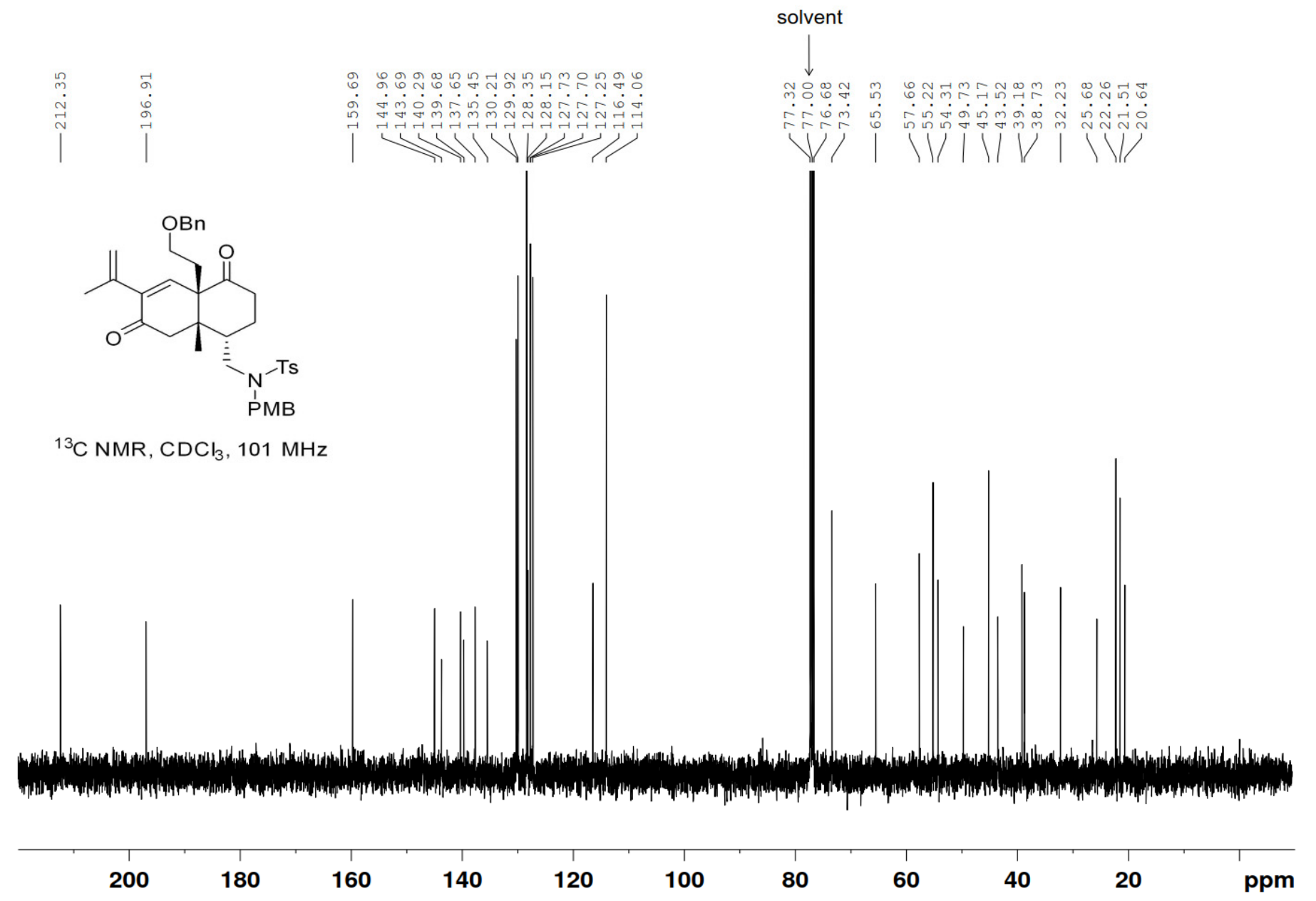


${ }^{1} \mathrm{H}$ NMR of $( \pm)-\mathbf{S 1}$

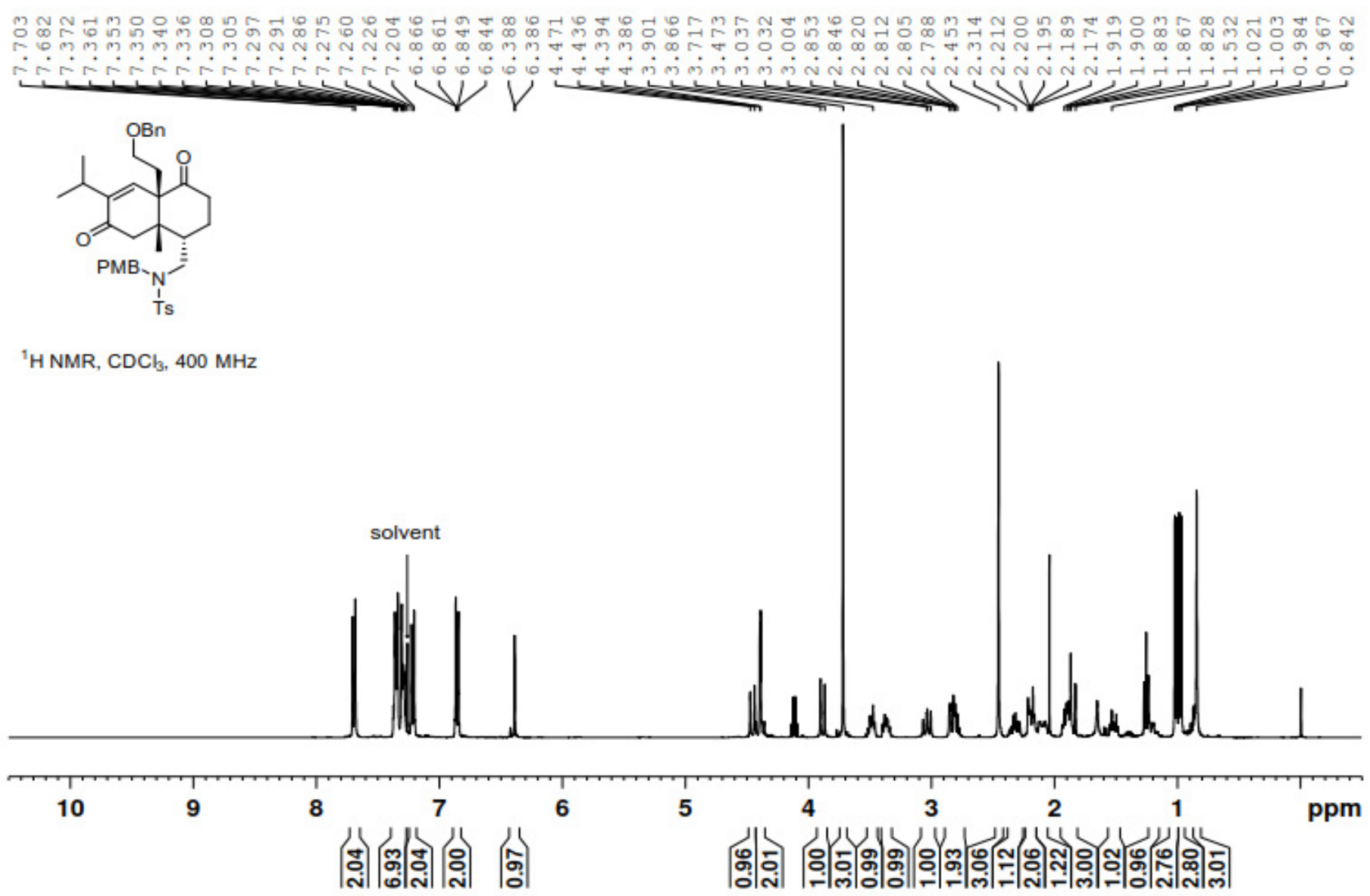


${ }^{13} \mathrm{C}$ NMR of $( \pm)-\mathbf{S 1}$
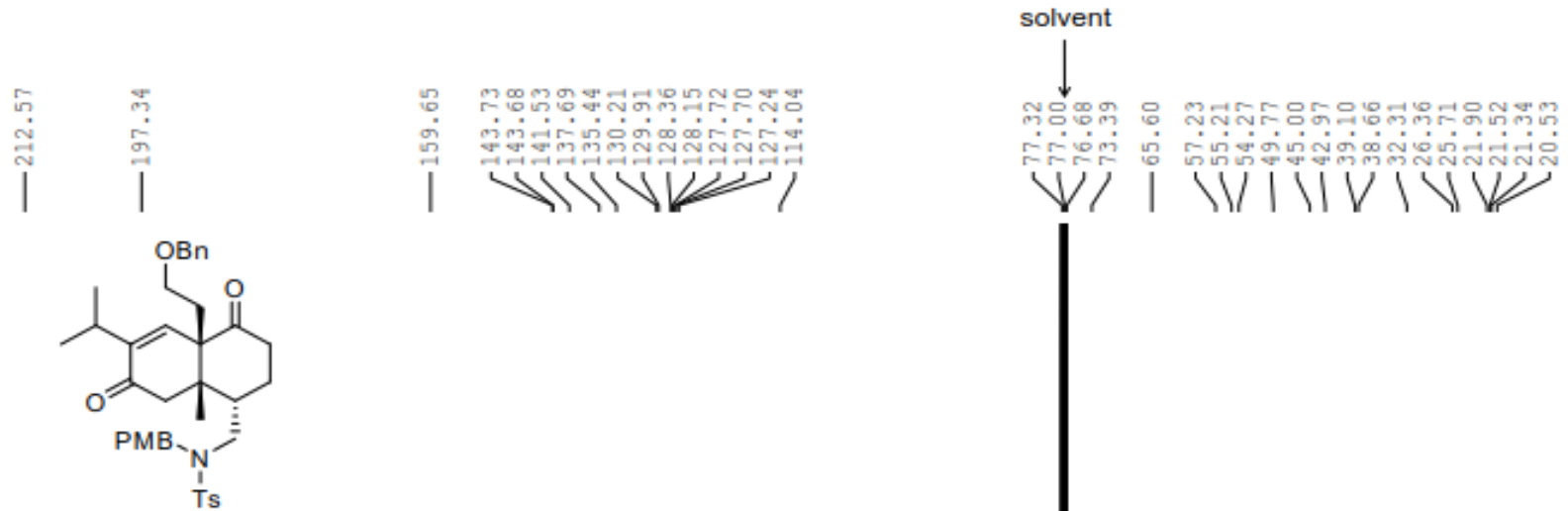

${ }^{13} \mathrm{C}$ NMR, $\mathrm{CDCl}_{3}, 101 \mathrm{MHz}$

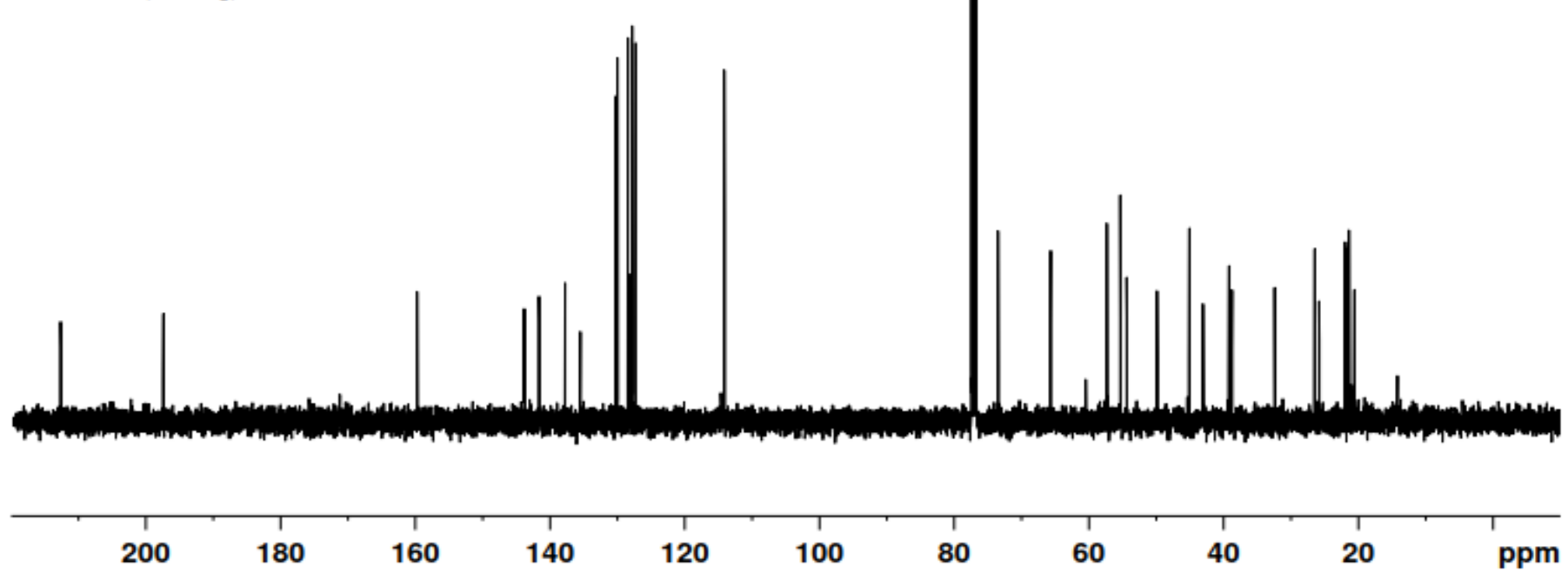




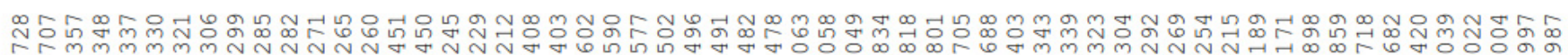

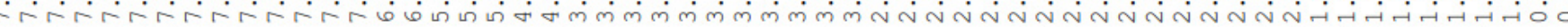

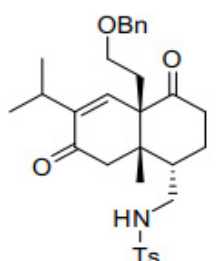

${ }^{1} \mathrm{H} \mathrm{NMR}, \mathrm{CDCl}_{3}, 400 \mathrm{MHz}$

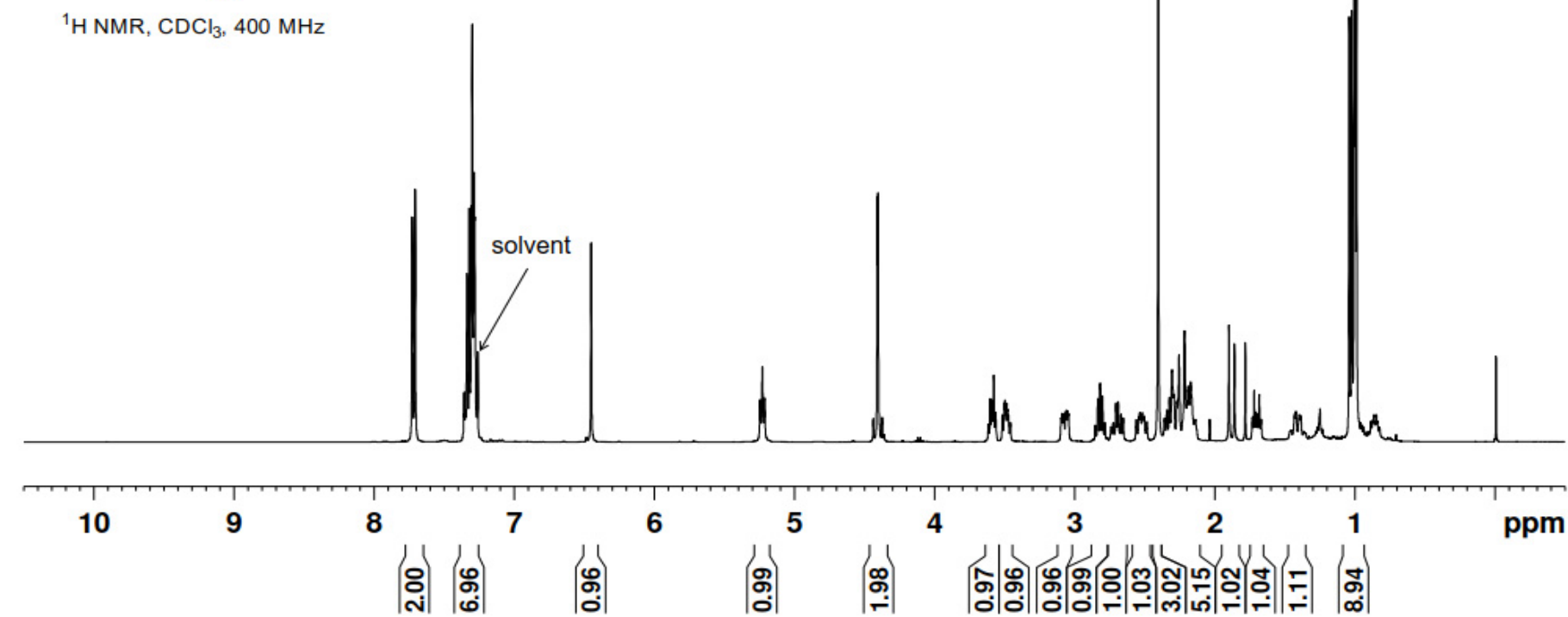


${ }^{13} \mathrm{C}$ NMR of $( \pm)-20$

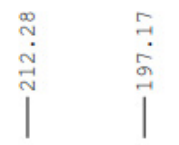

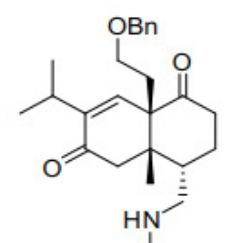

${ }^{13} \mathrm{C} \mathrm{NMR}, \mathrm{CDCl}_{3}, 101 \mathrm{MHz}$
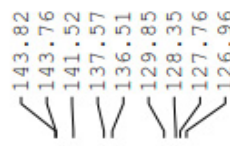

solvent
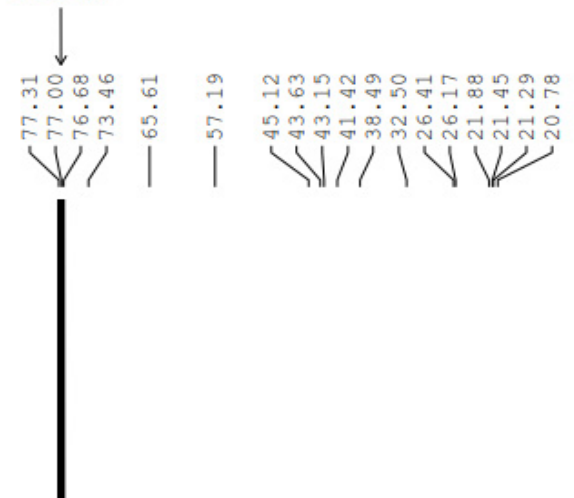
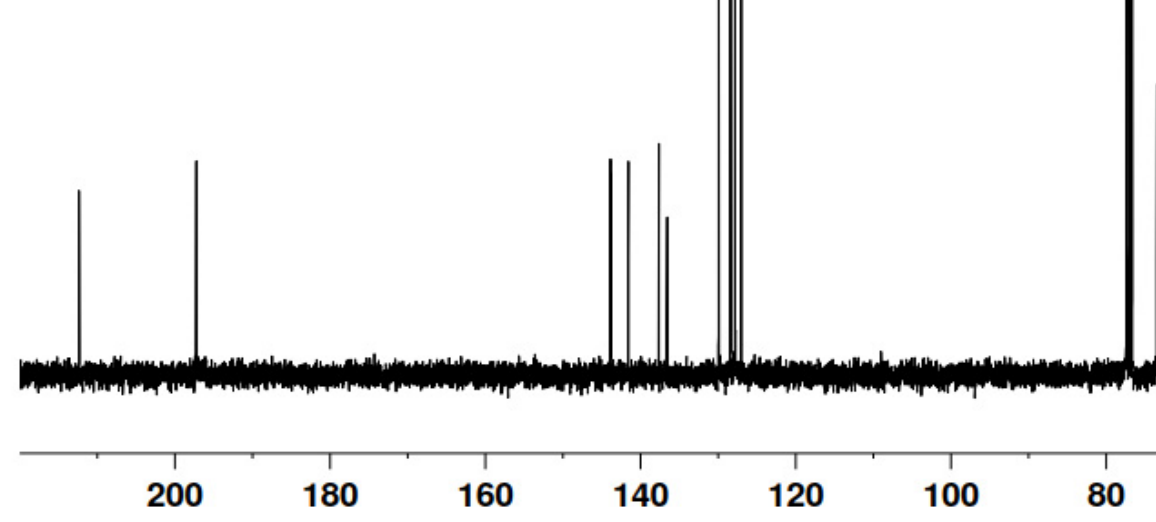

60

40

ppm 
${ }^{1} \mathrm{H}$ NMR of $( \pm)-22$

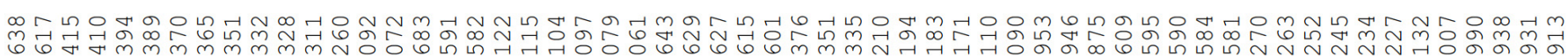

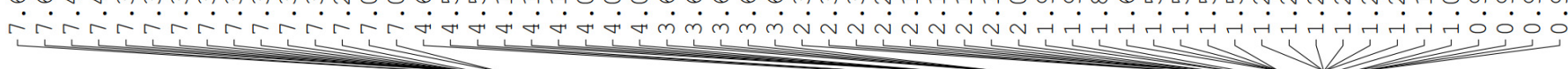

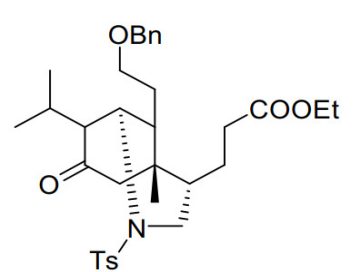

${ }^{1} \mathrm{HNMR}, \mathrm{CDCl}_{3}, 400 \mathrm{MHz}$

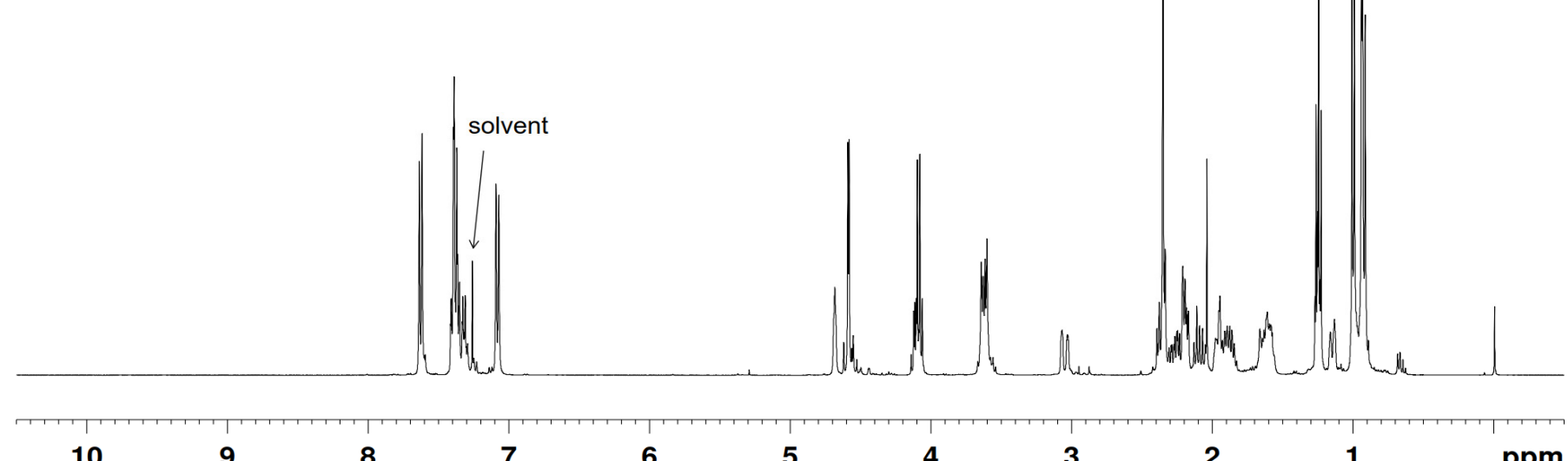

10

8

7

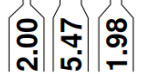

5

4

2

1

ppm

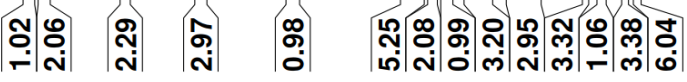


${ }^{13} \mathrm{C}$ NMR of $( \pm)-22$

$$
\text { I }
$$
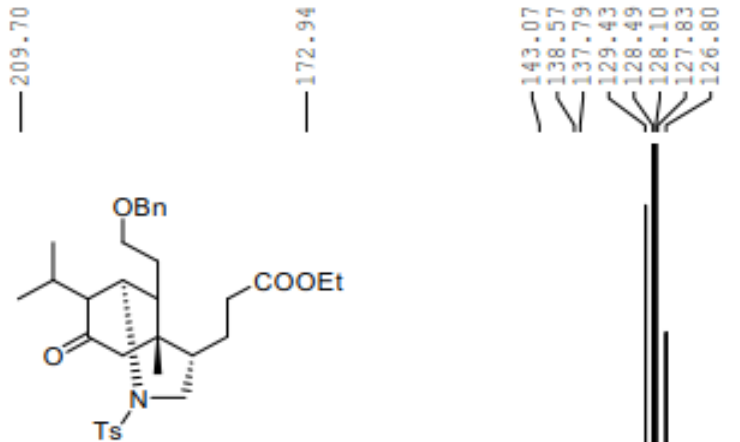

${ }^{13} \mathrm{C} \mathrm{NMR}, \mathrm{CDCl}_{3}, 101 \mathrm{MHz}$

$$
\text { solvent }
$$

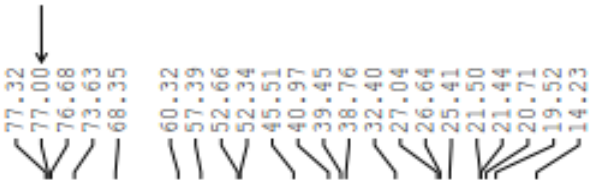

v/
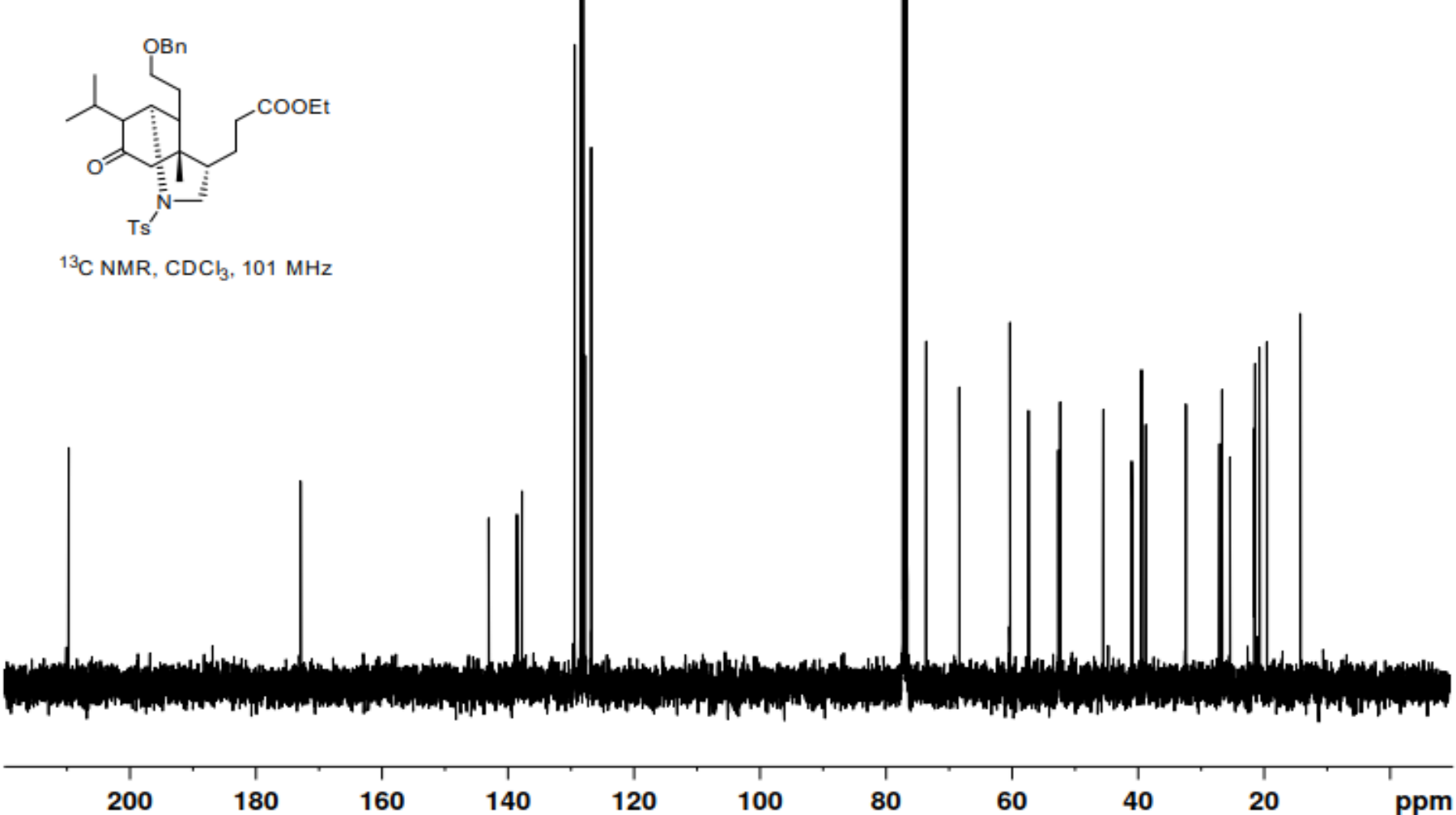
${ }^{1} \mathrm{H}$ NMR of $( \pm)-23$

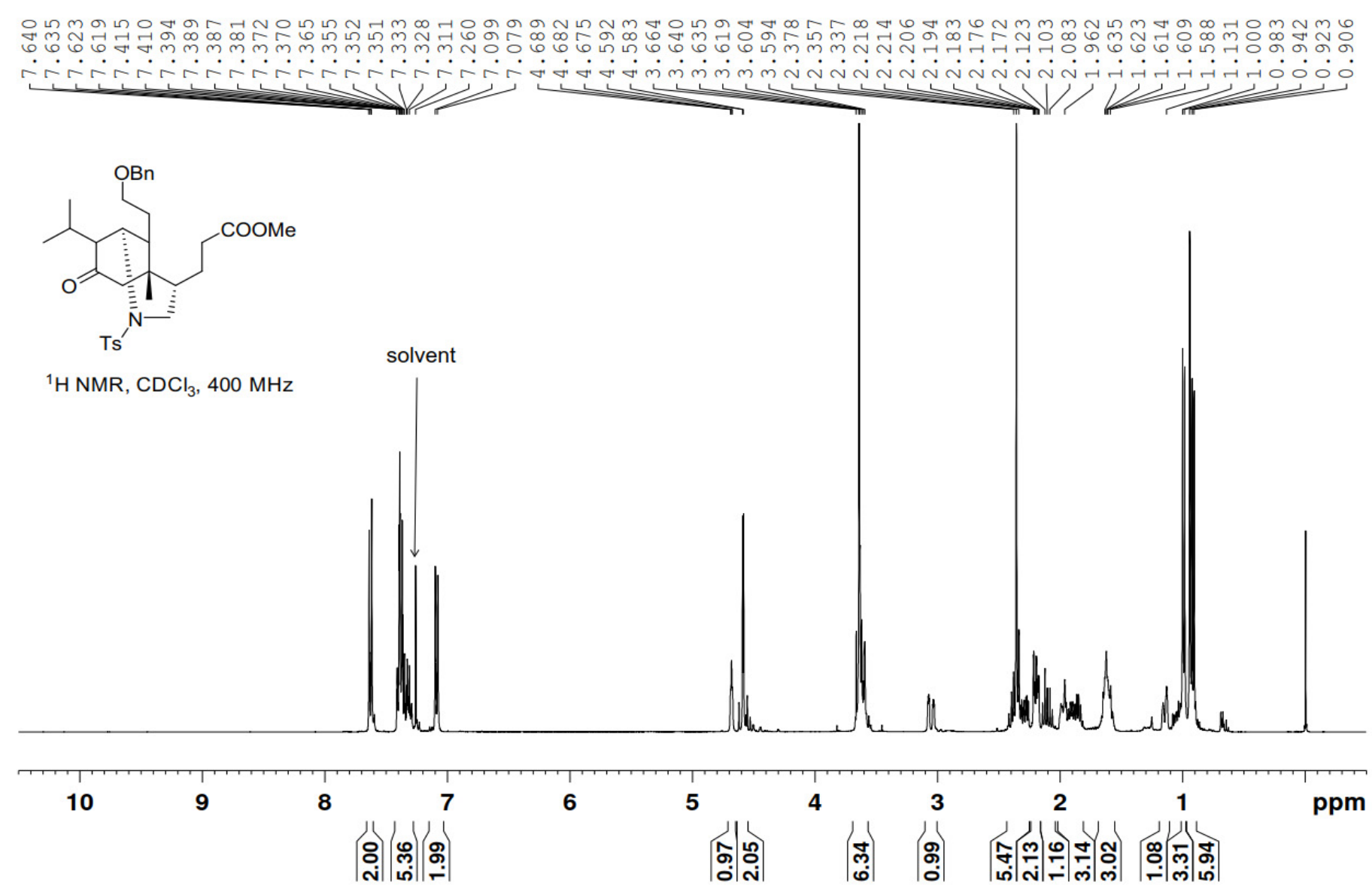


${ }^{13} \mathrm{C}$ NMR of $( \pm)-23$

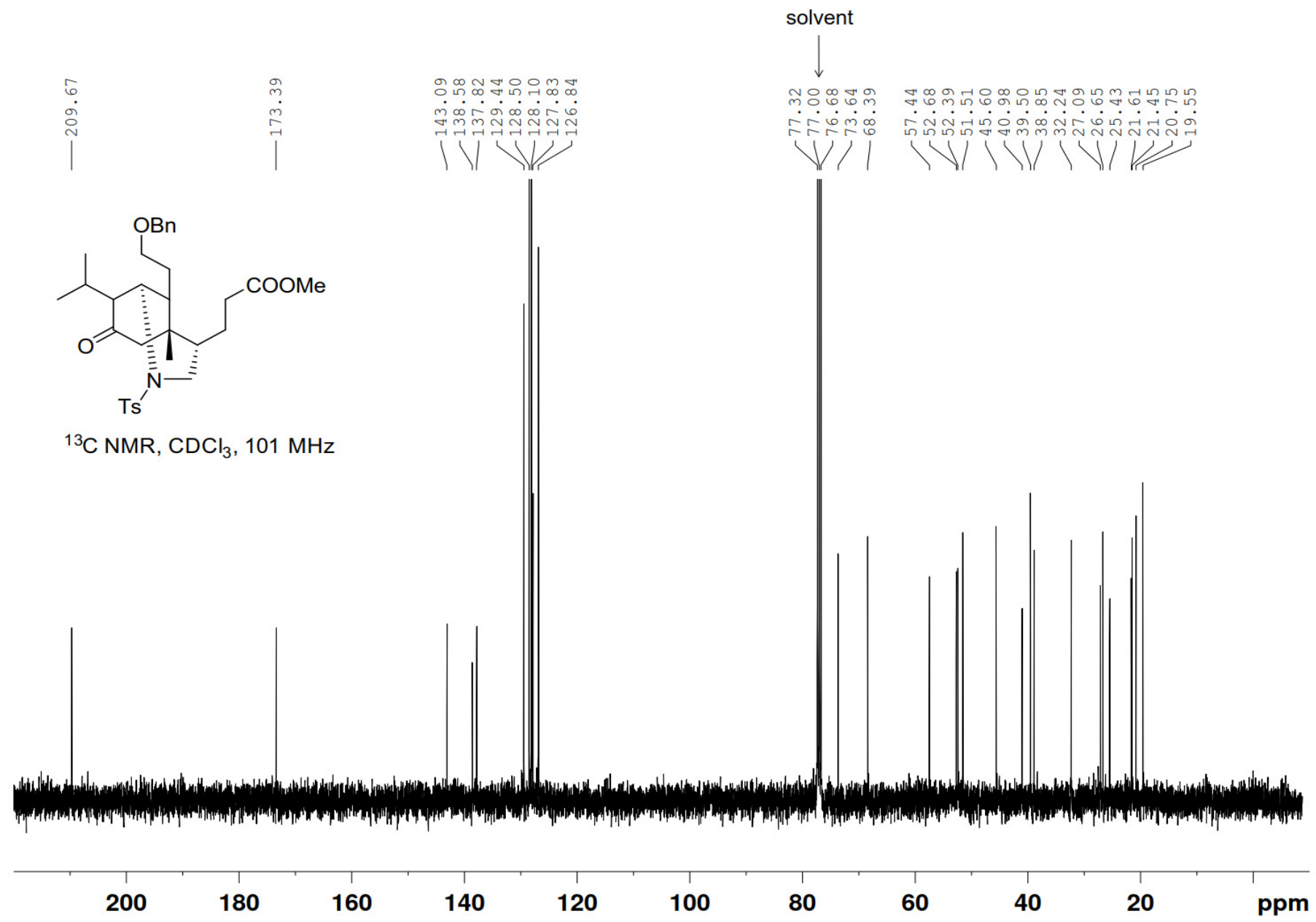



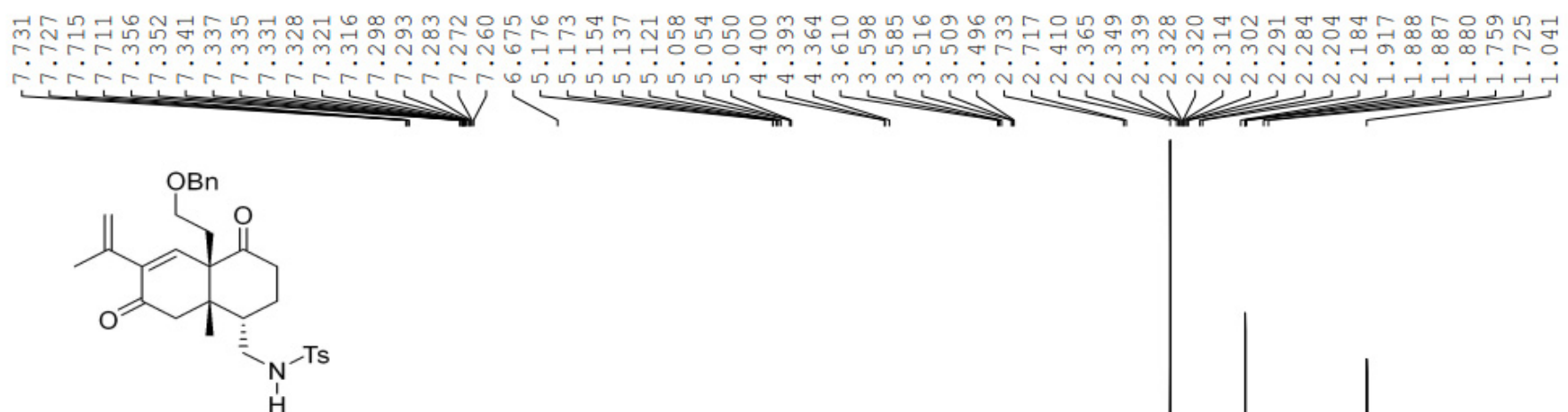

${ }^{1} \mathrm{H}$ NMR, $\mathrm{CDC}_{3}, 400 \mathrm{MHz}$

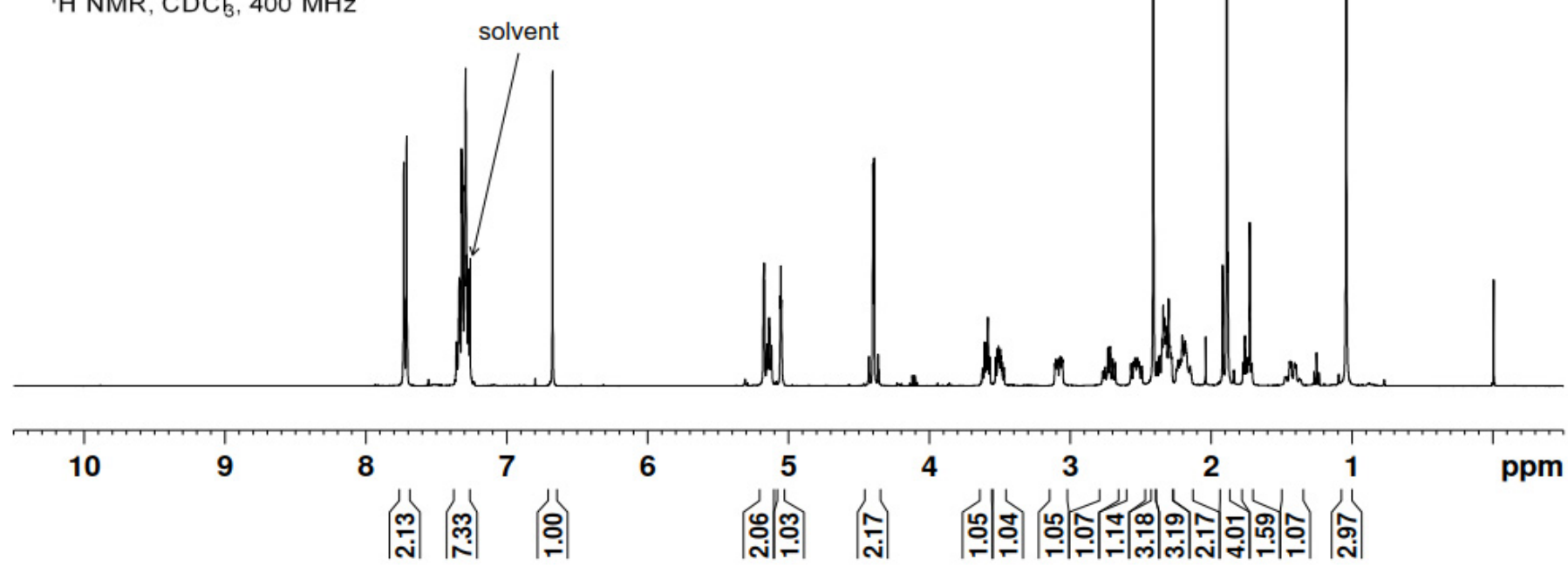


${ }^{13} \mathrm{C}$ NMR of 4

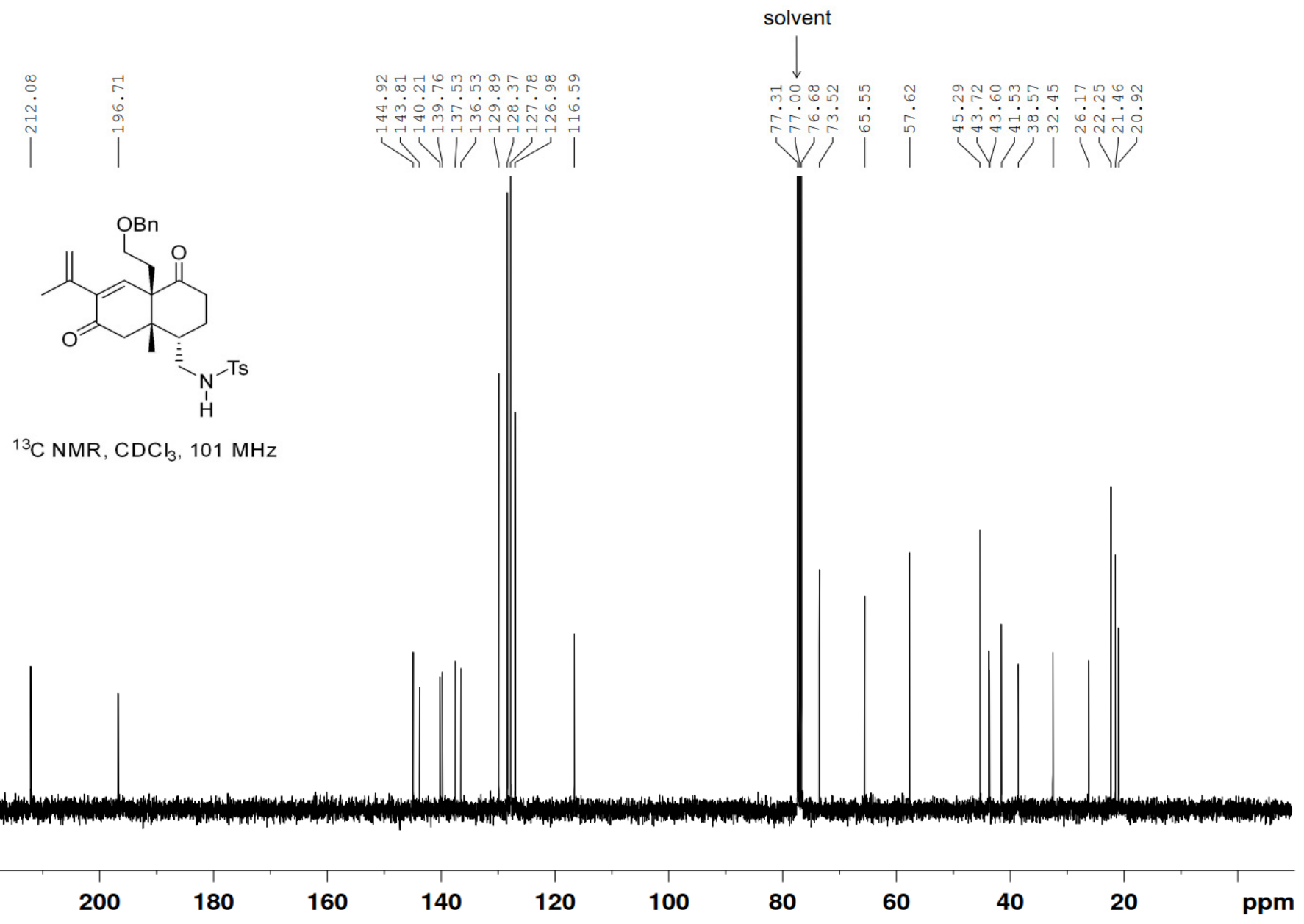




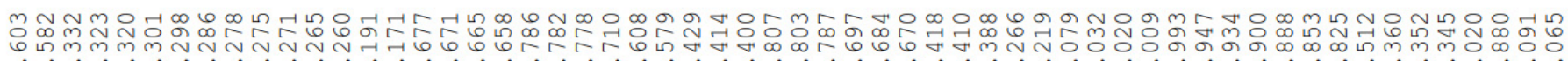
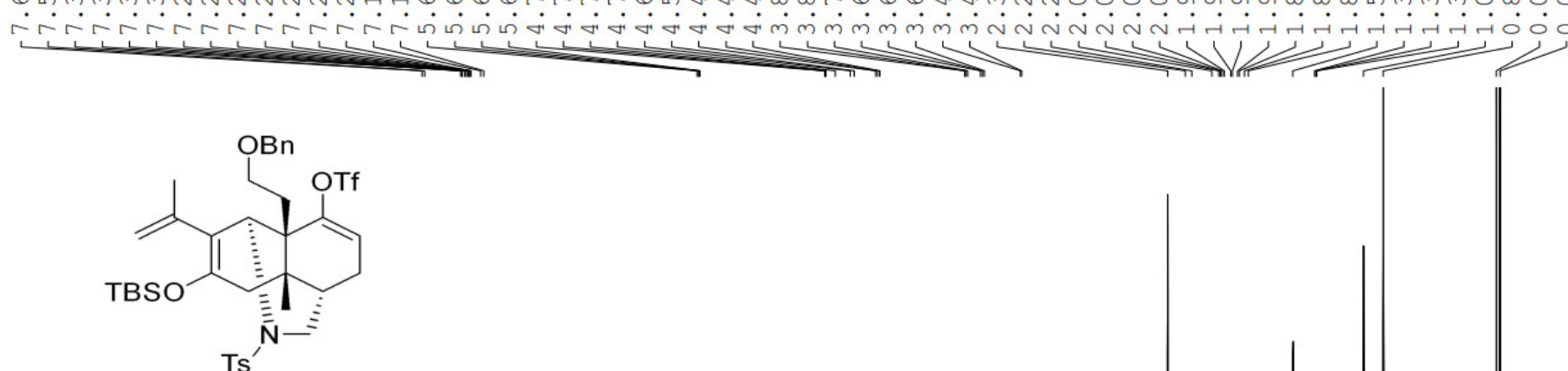

${ }^{1} \mathrm{H} \mathrm{NMR}, \mathrm{CDC}_{3}, 400 \mathrm{MHz}$
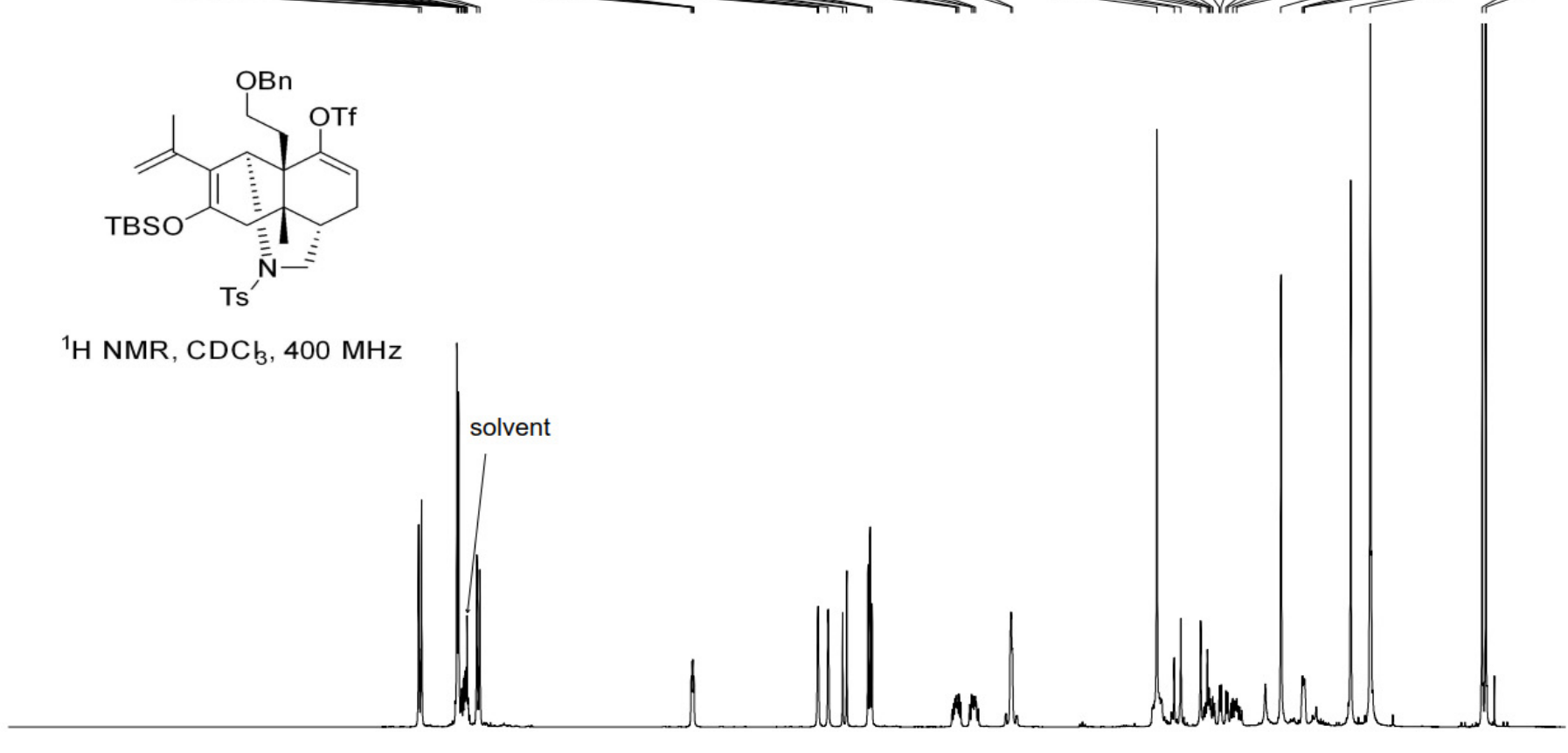

10 8 7

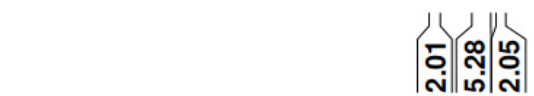
Alh

|ல்

3 2 1

ppm 
${ }^{13} \mathrm{C}$ NMR of 24

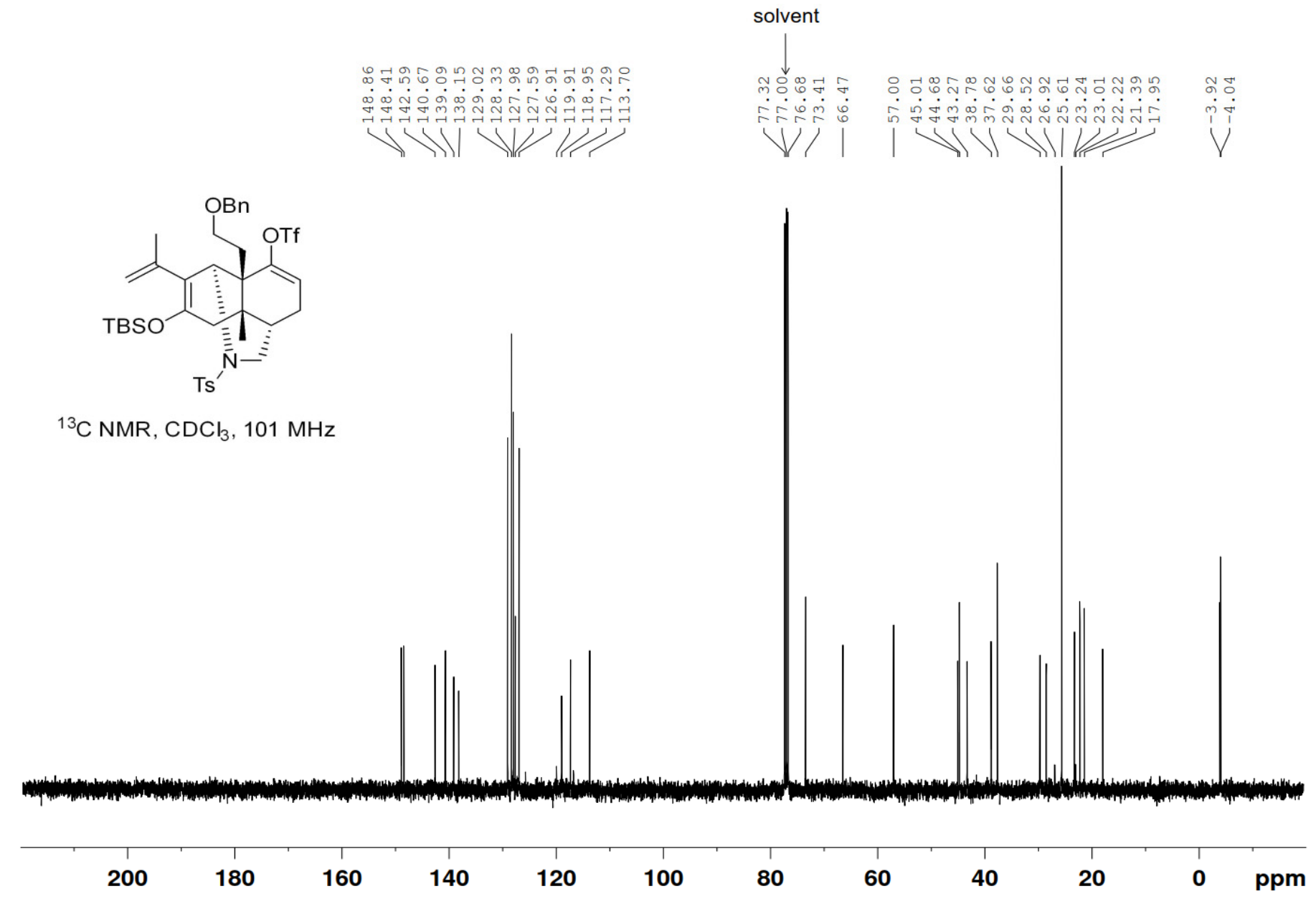




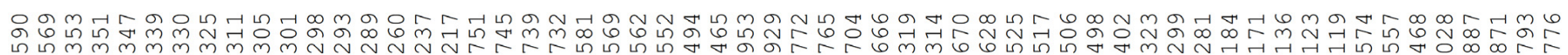

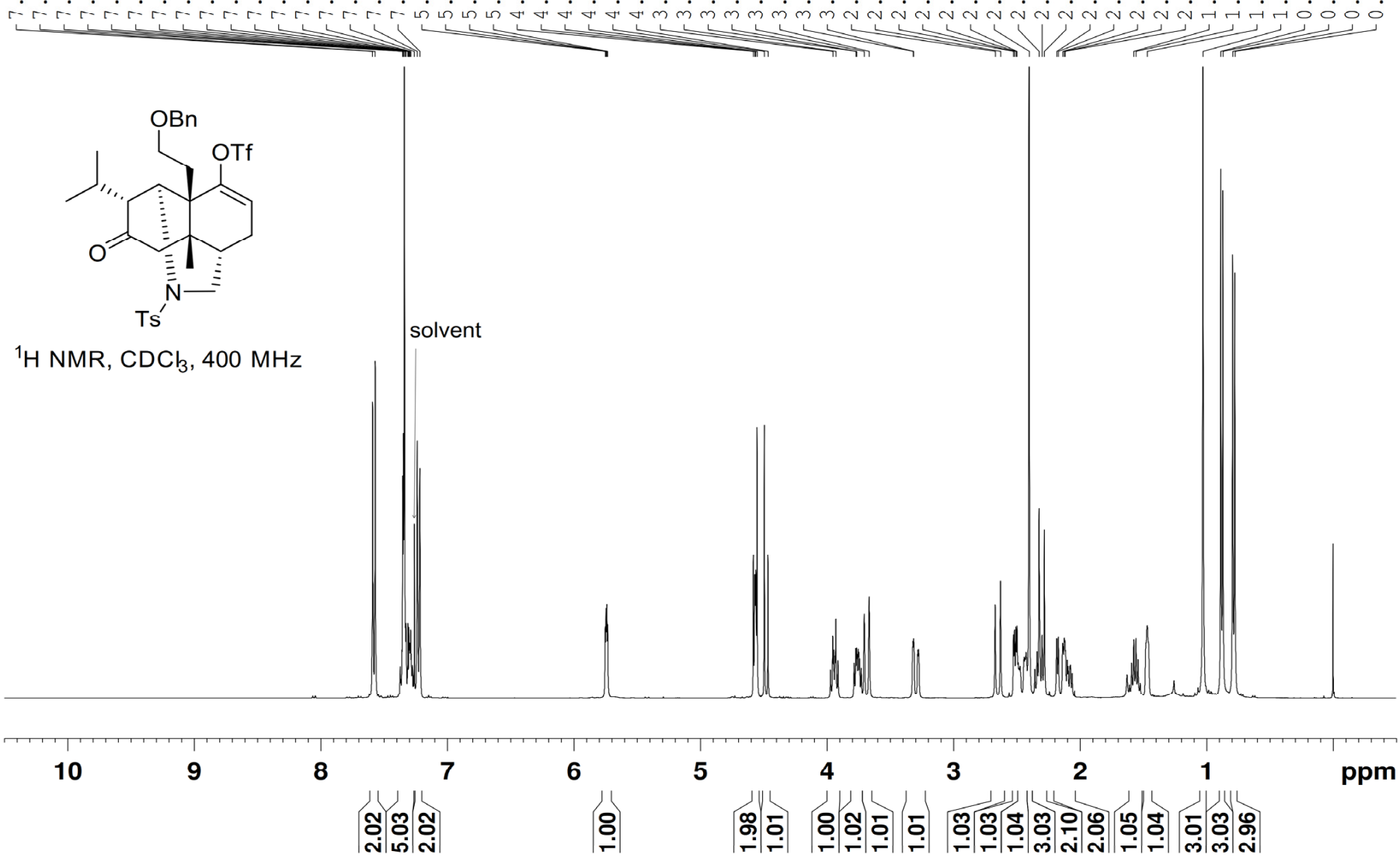


${ }^{13} \mathrm{C}$ NMR of 25

ㄱ.

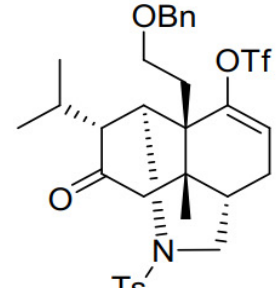

${ }^{13} \mathrm{C} \mathrm{NMR}, \mathrm{CDCl}_{3}, 101 \mathrm{MHz}$

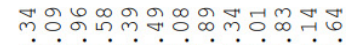

क mim

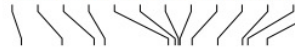

|

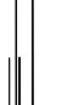

solvent

$\downarrow$

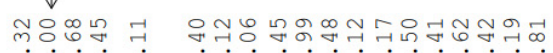

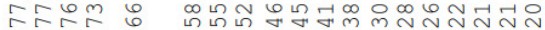

V)

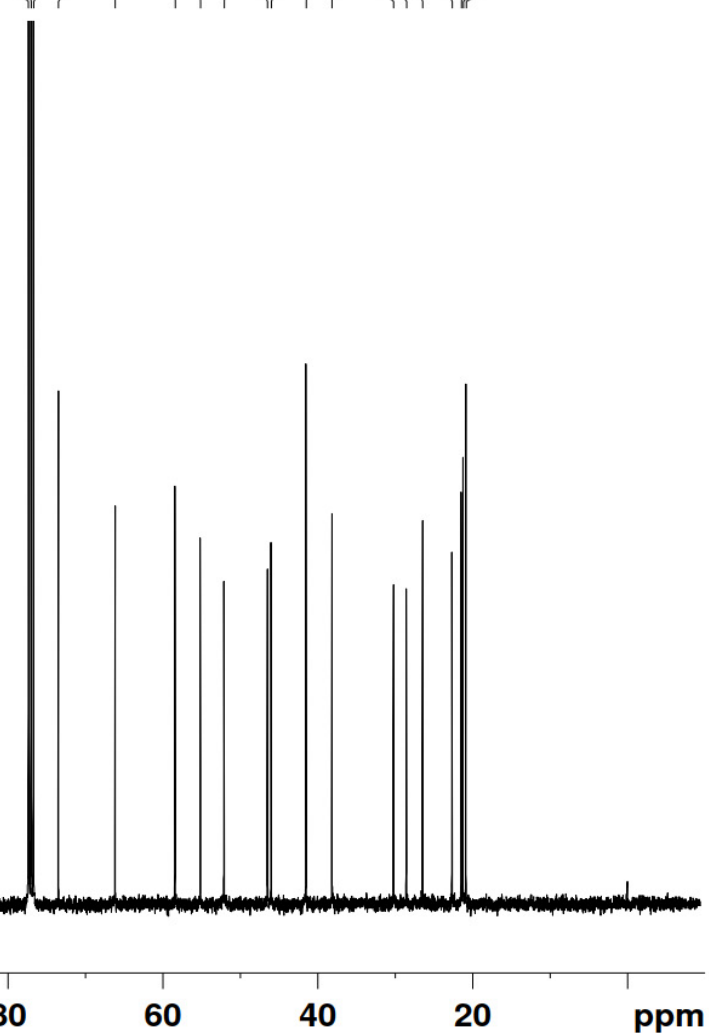




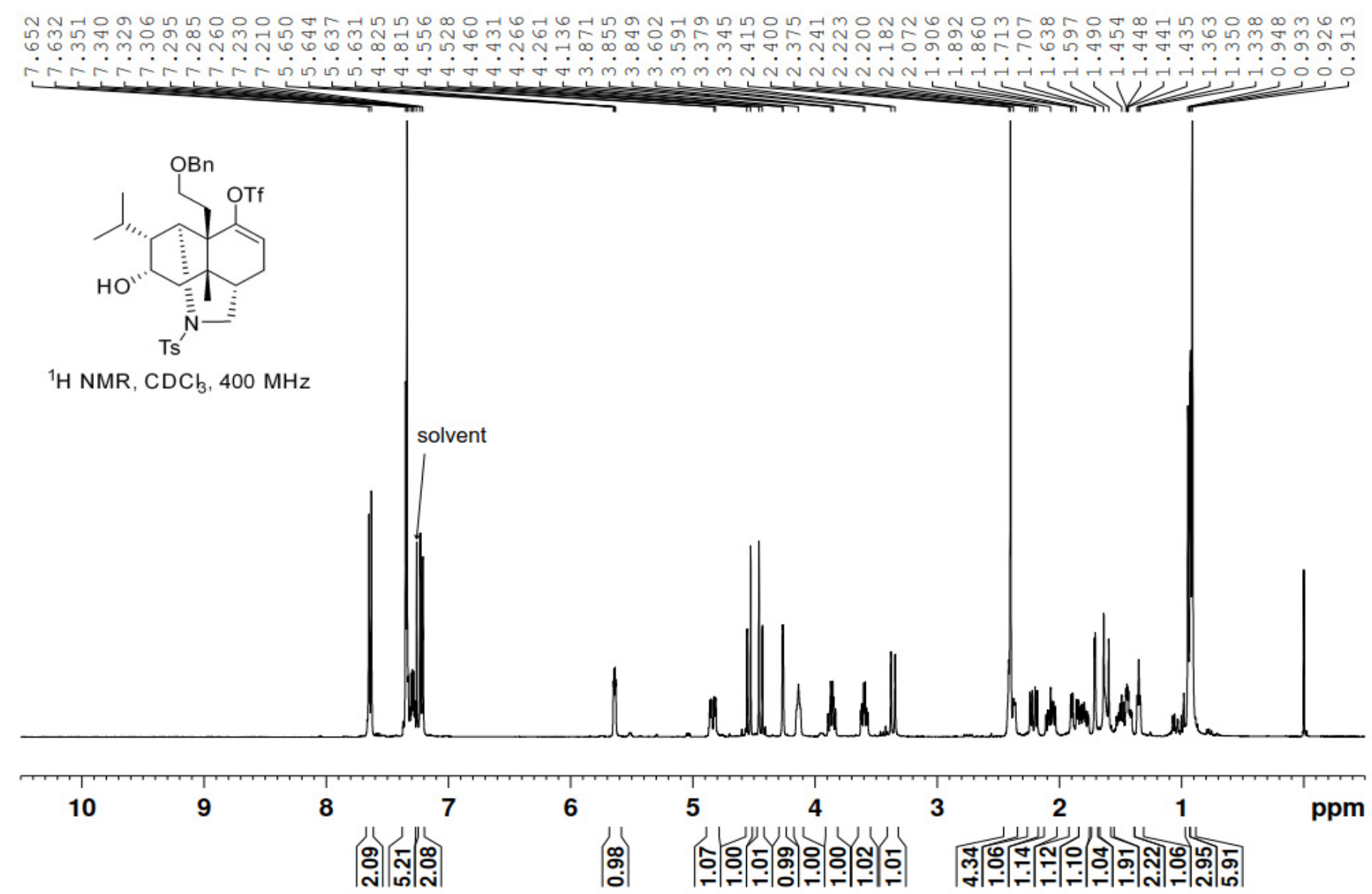


${ }^{13} \mathrm{C}$ NMR of $\mathbf{S 2}$

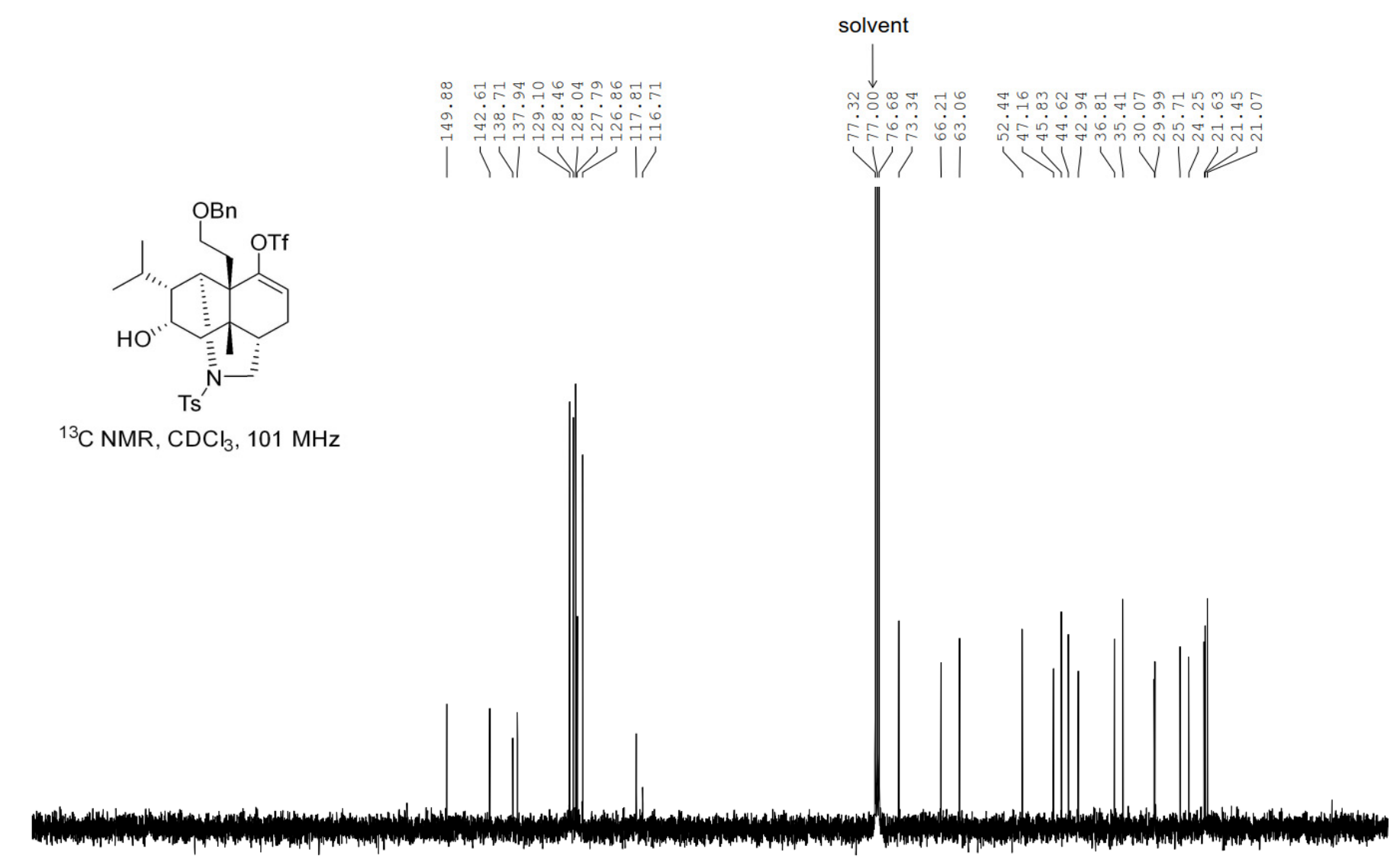

160

120

100

80

60

40

20

ppm 


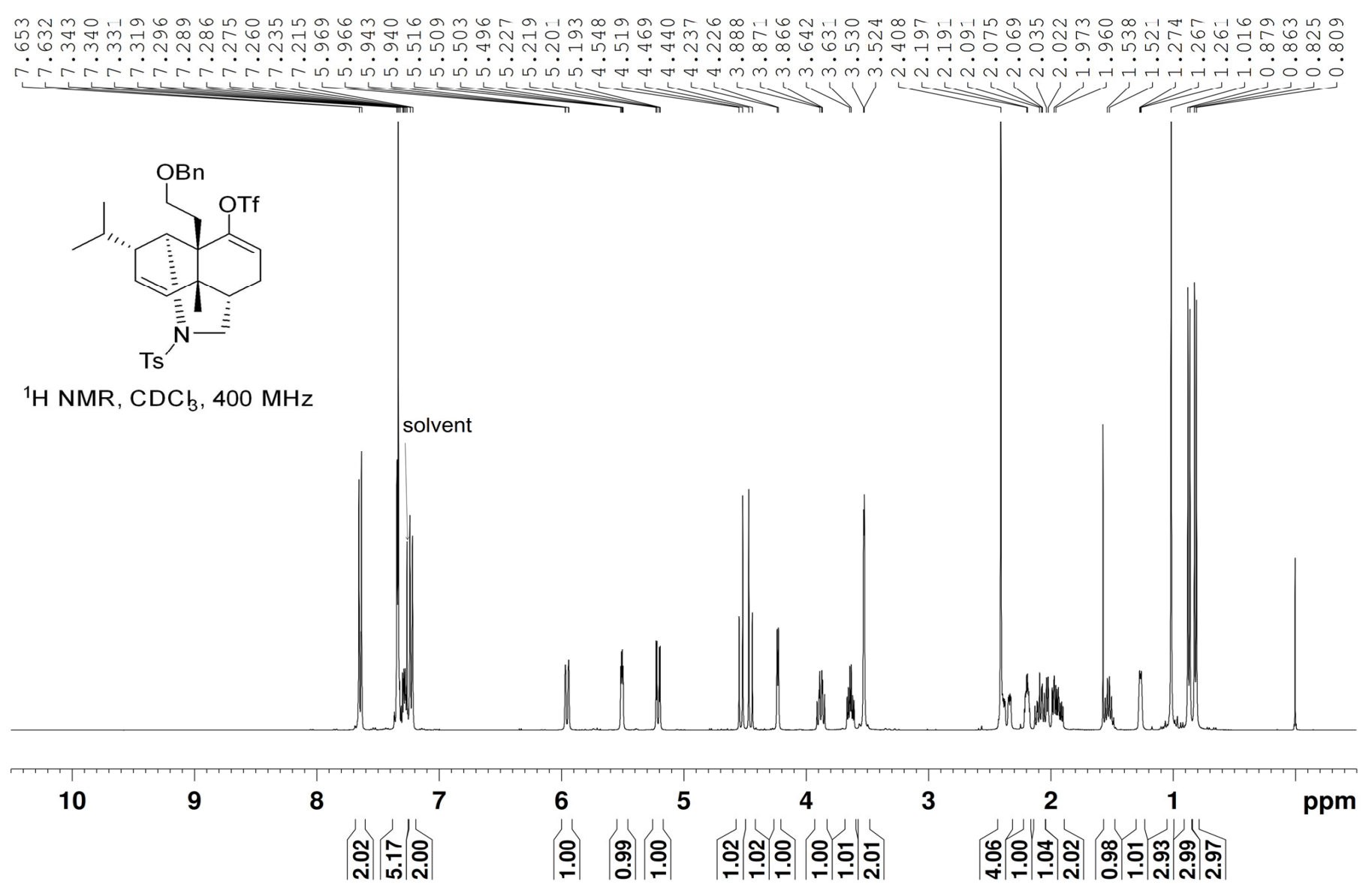


${ }^{13} \mathrm{C}$ NMR of 26

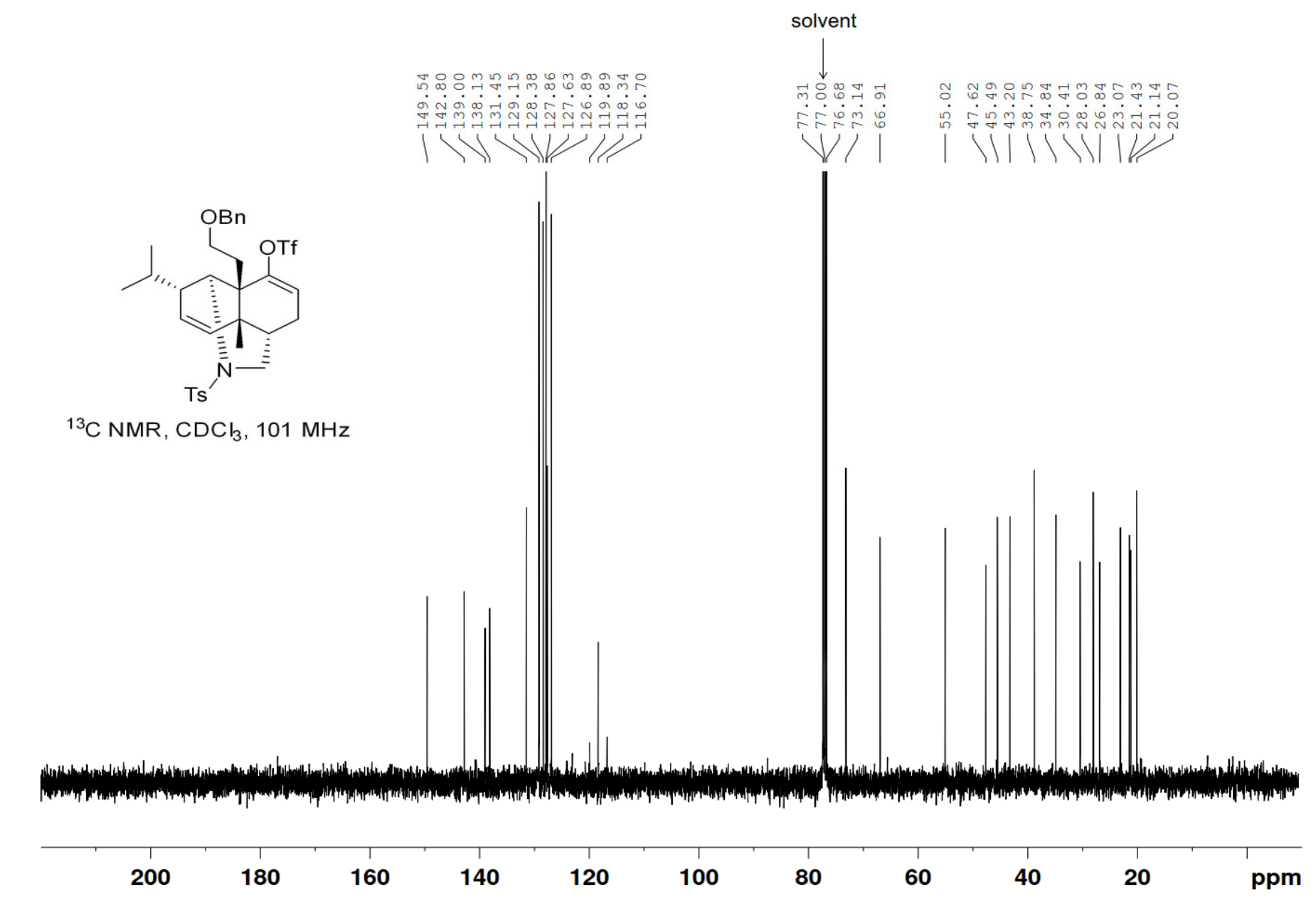




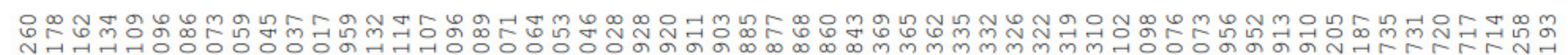

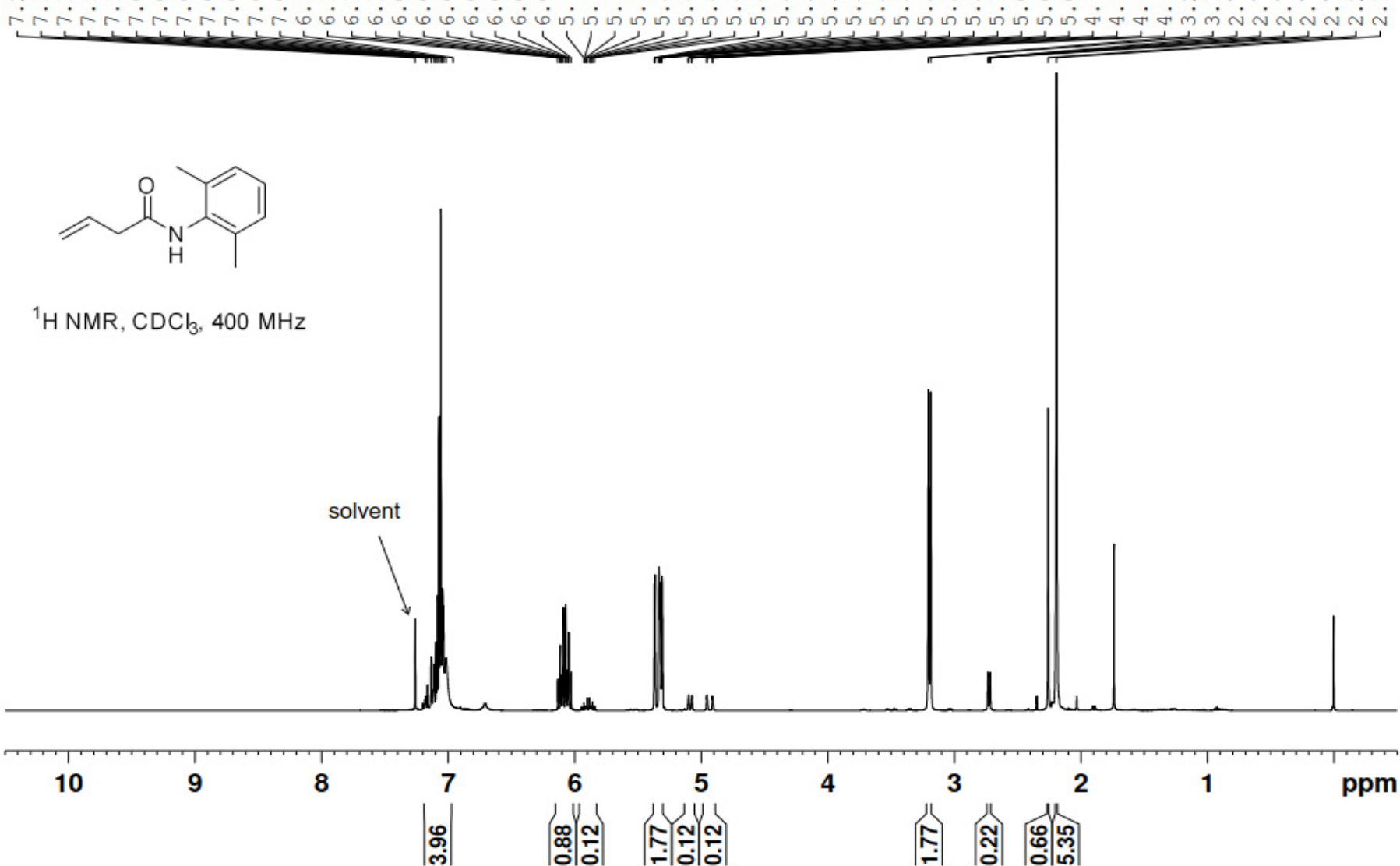


${ }^{13} \mathrm{C}$ NMR of 27

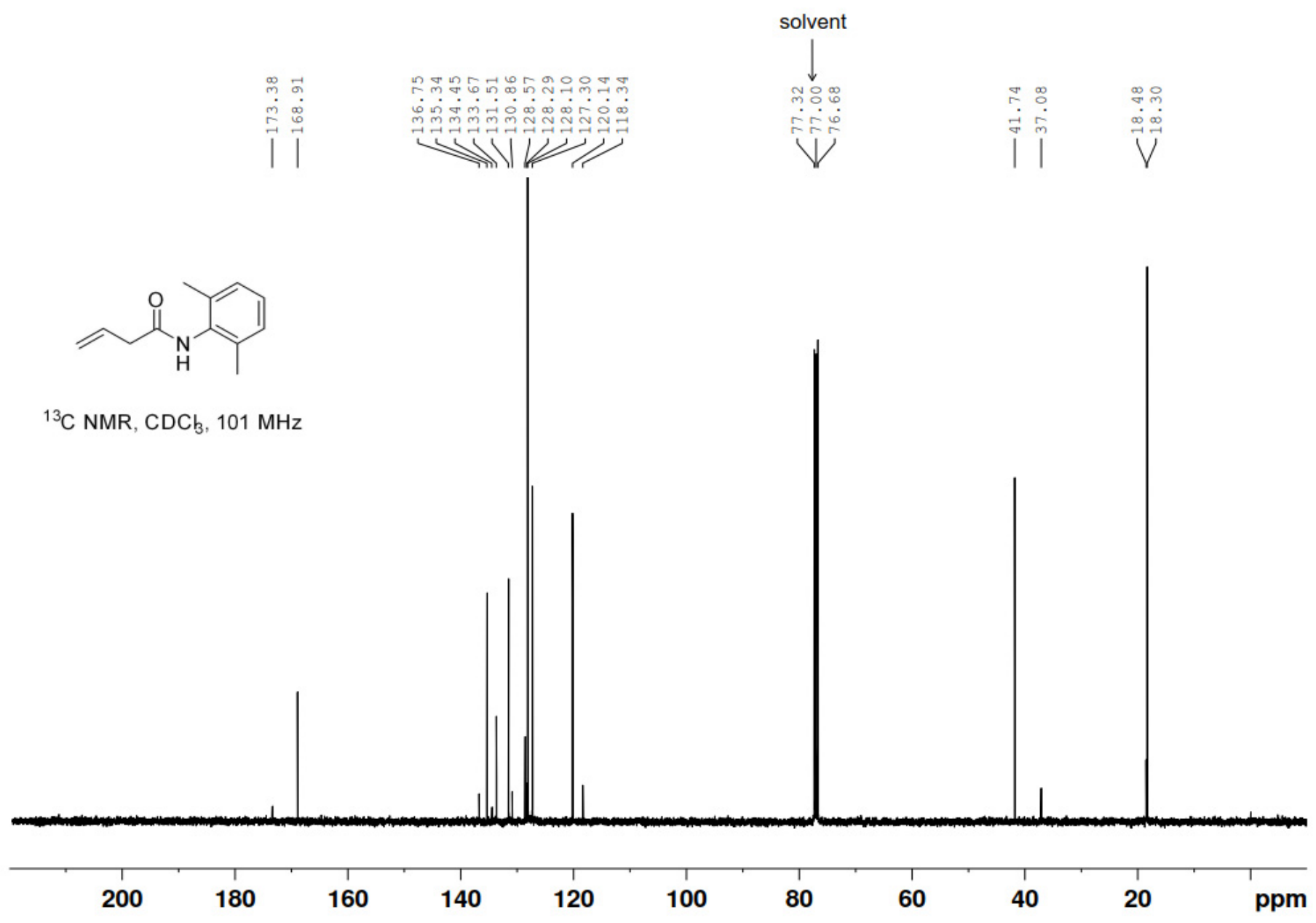




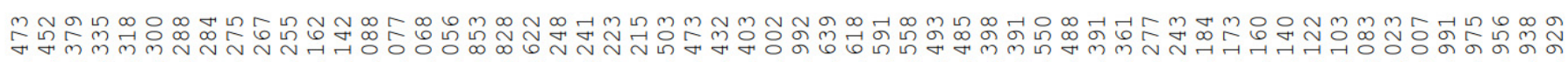

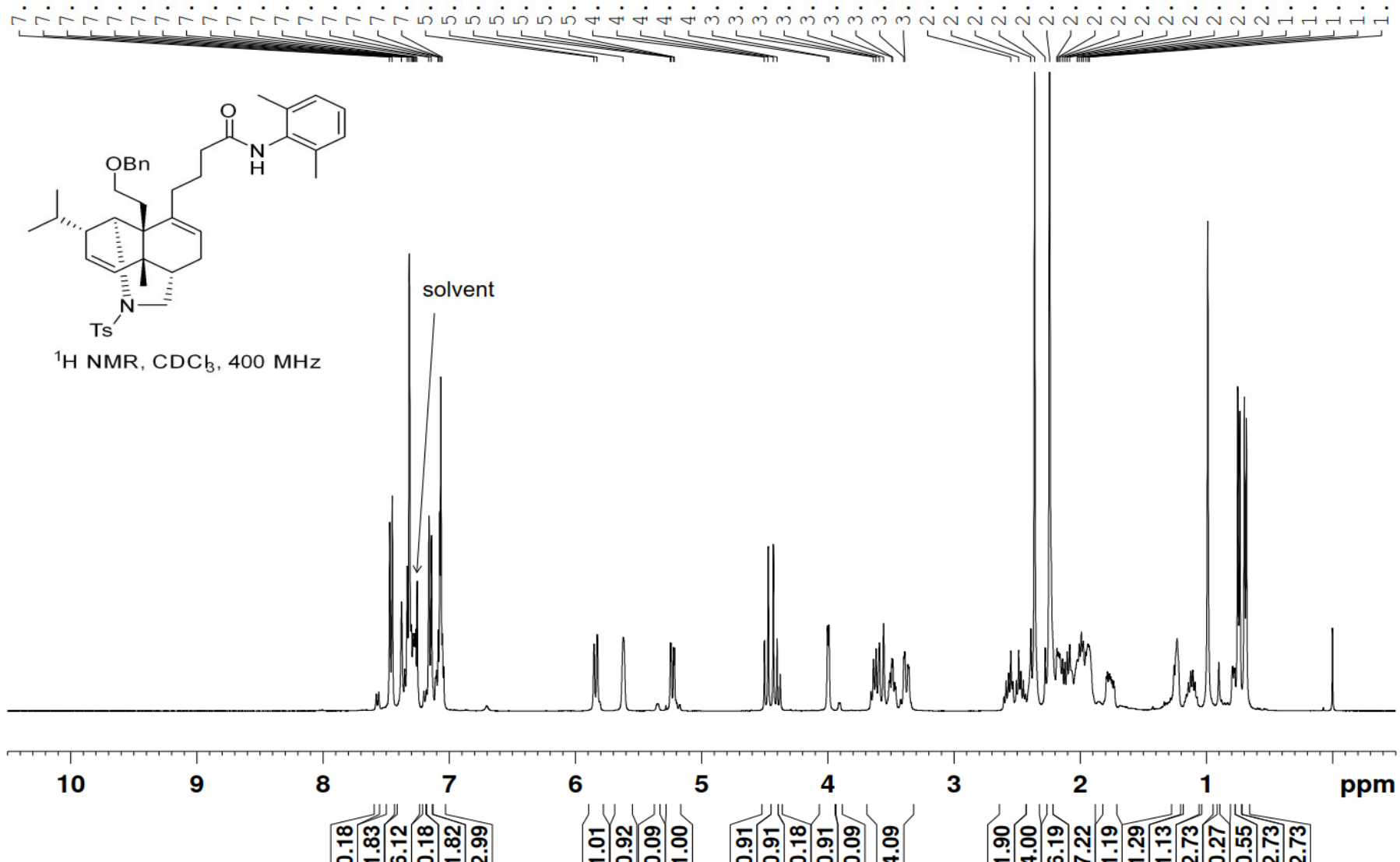

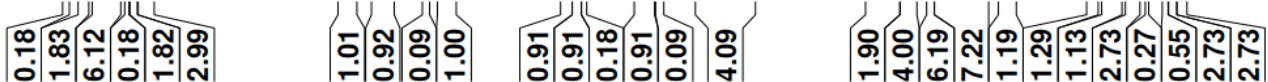


${ }^{13} \mathrm{C}$ NMR of 3

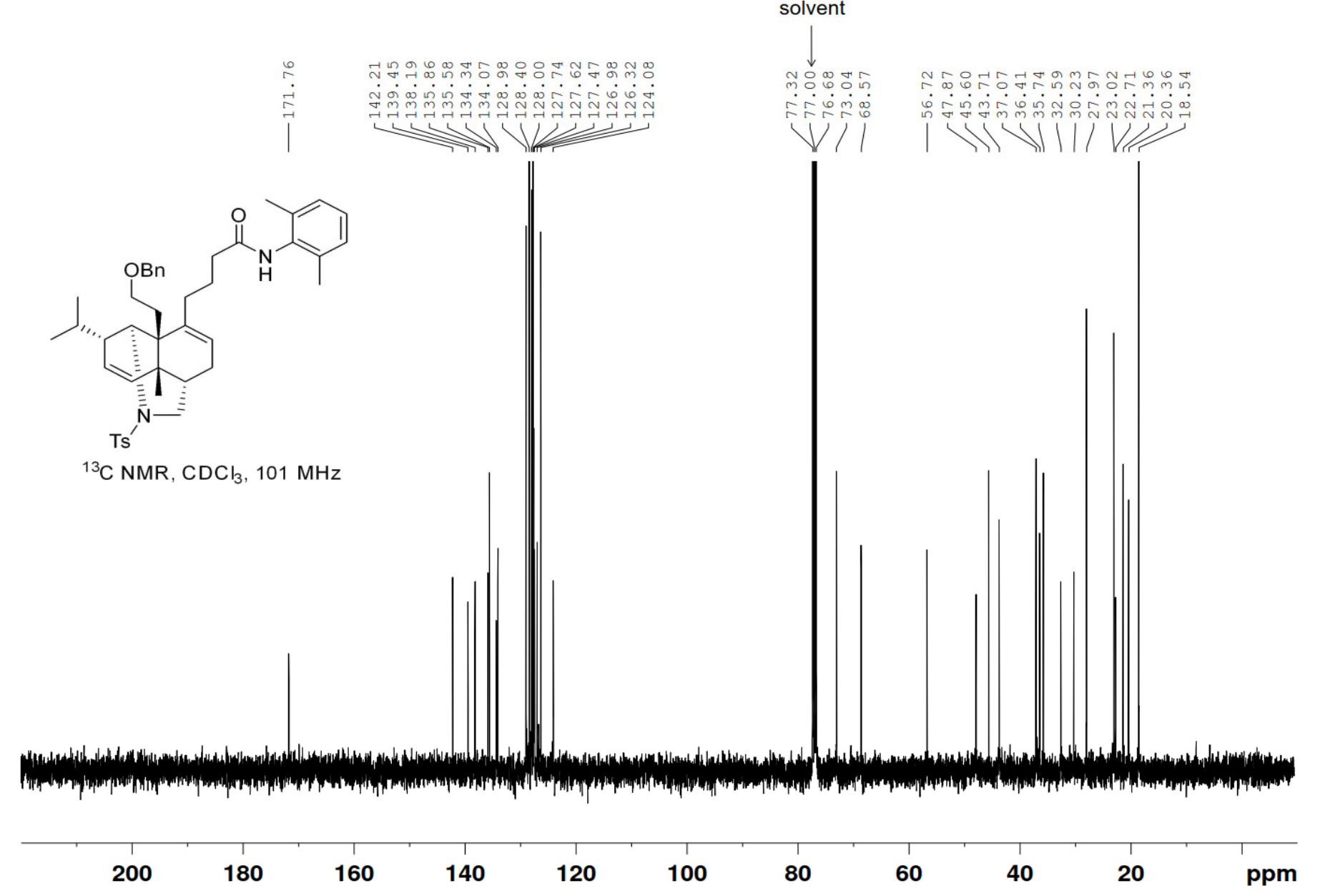




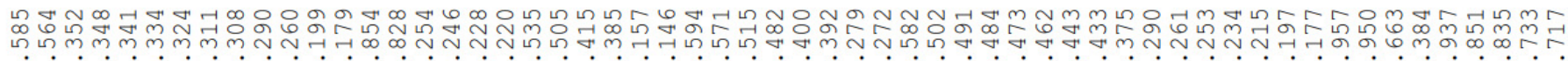

ririvir

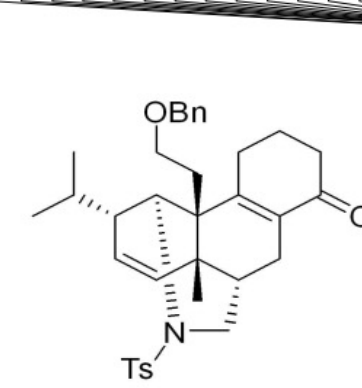

${ }^{1} \mathrm{H} N M R, \mathrm{CDCl}_{3}, 400 \mathrm{MHz}$
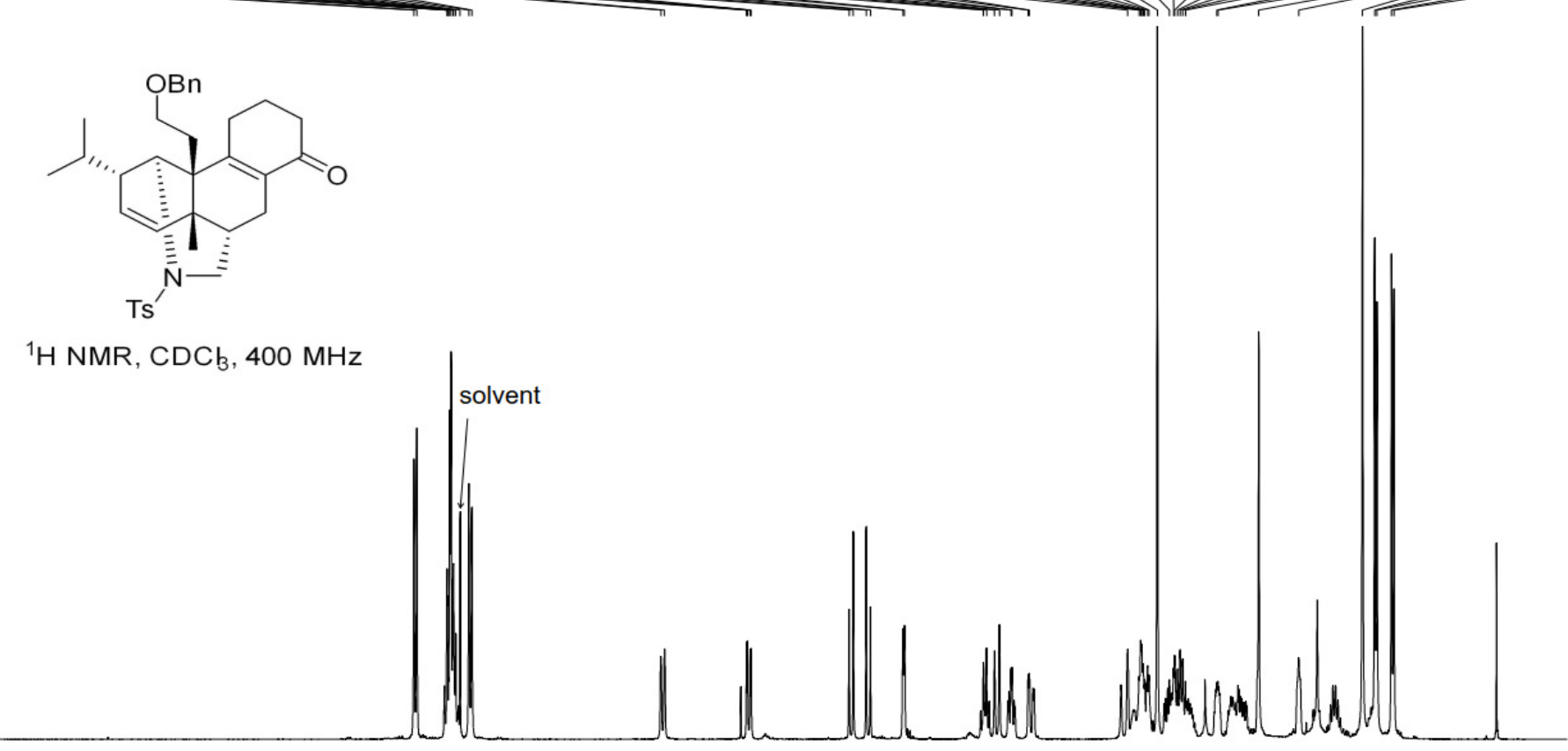

10 8 7

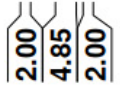
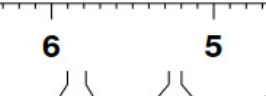
4 3 2 1 ppm | 
${ }^{13} \mathrm{C}$ NMR of 28

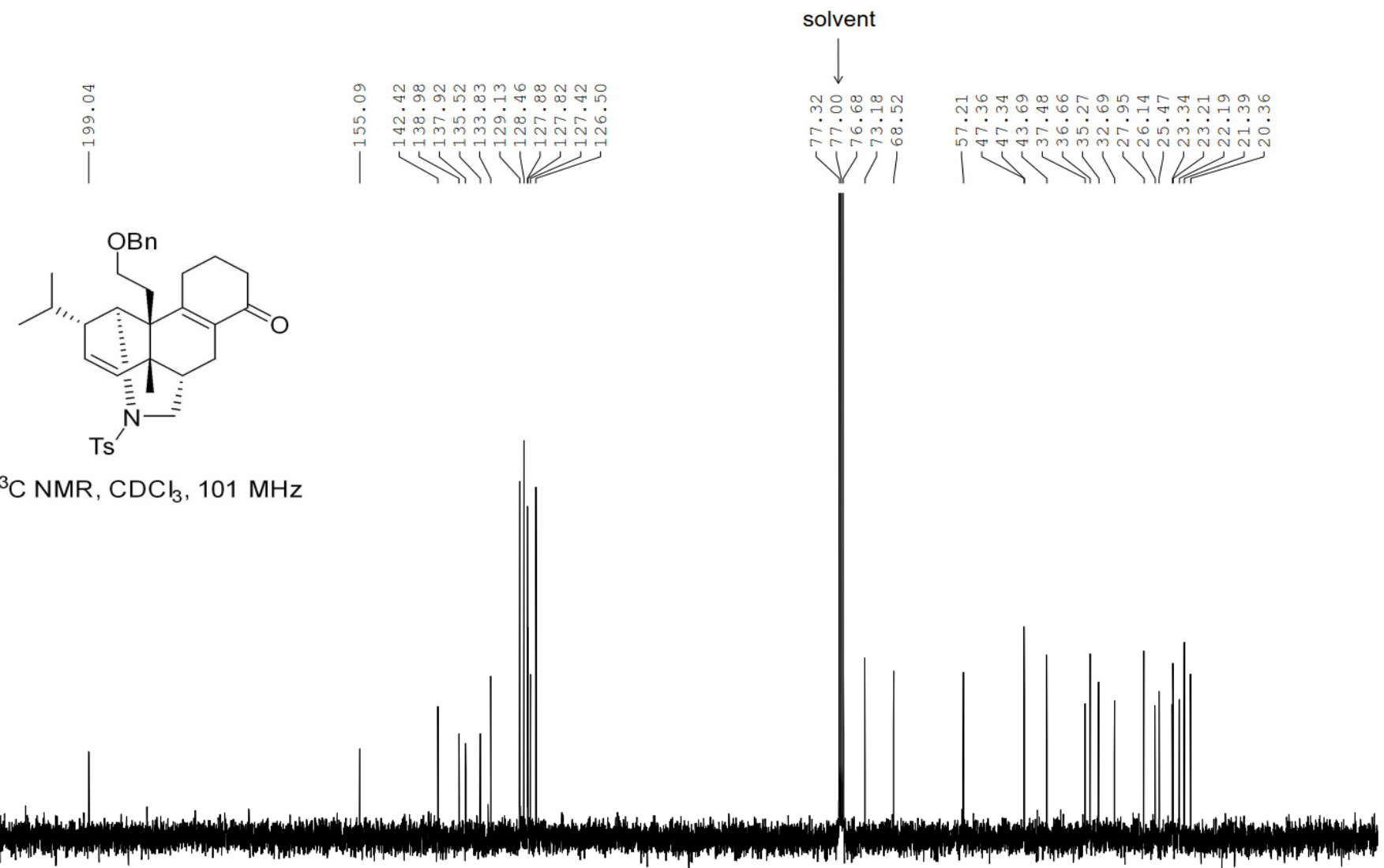

200

180

160

140

120

100

80

60

40

20

ppm 


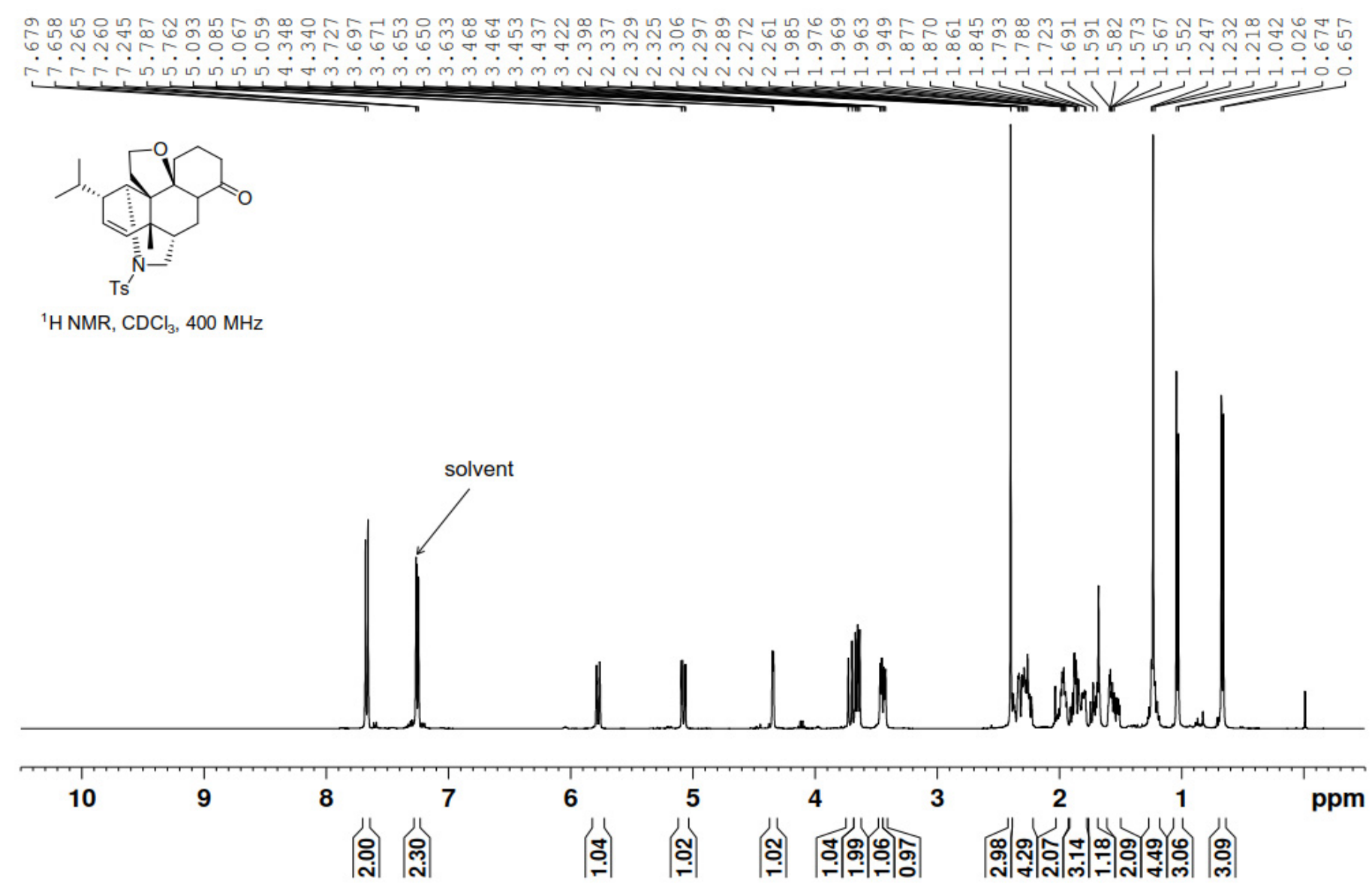


${ }^{13} \mathrm{C}$ NMR of 34

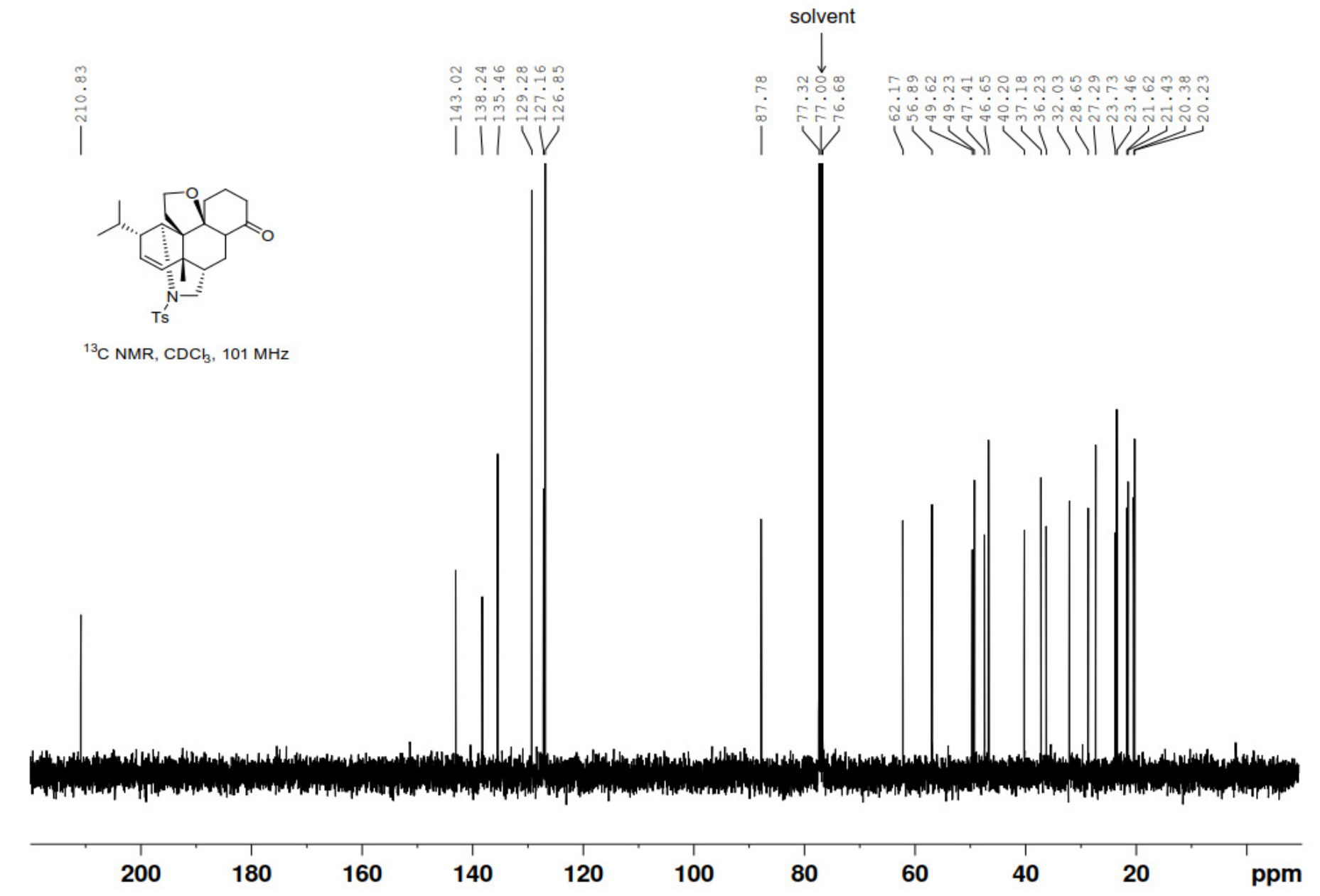




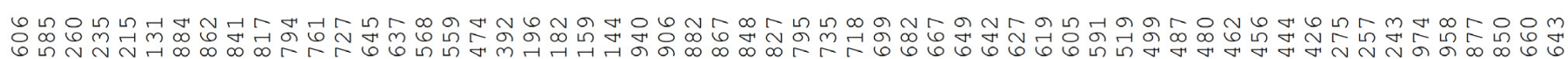

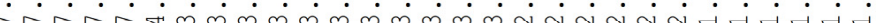

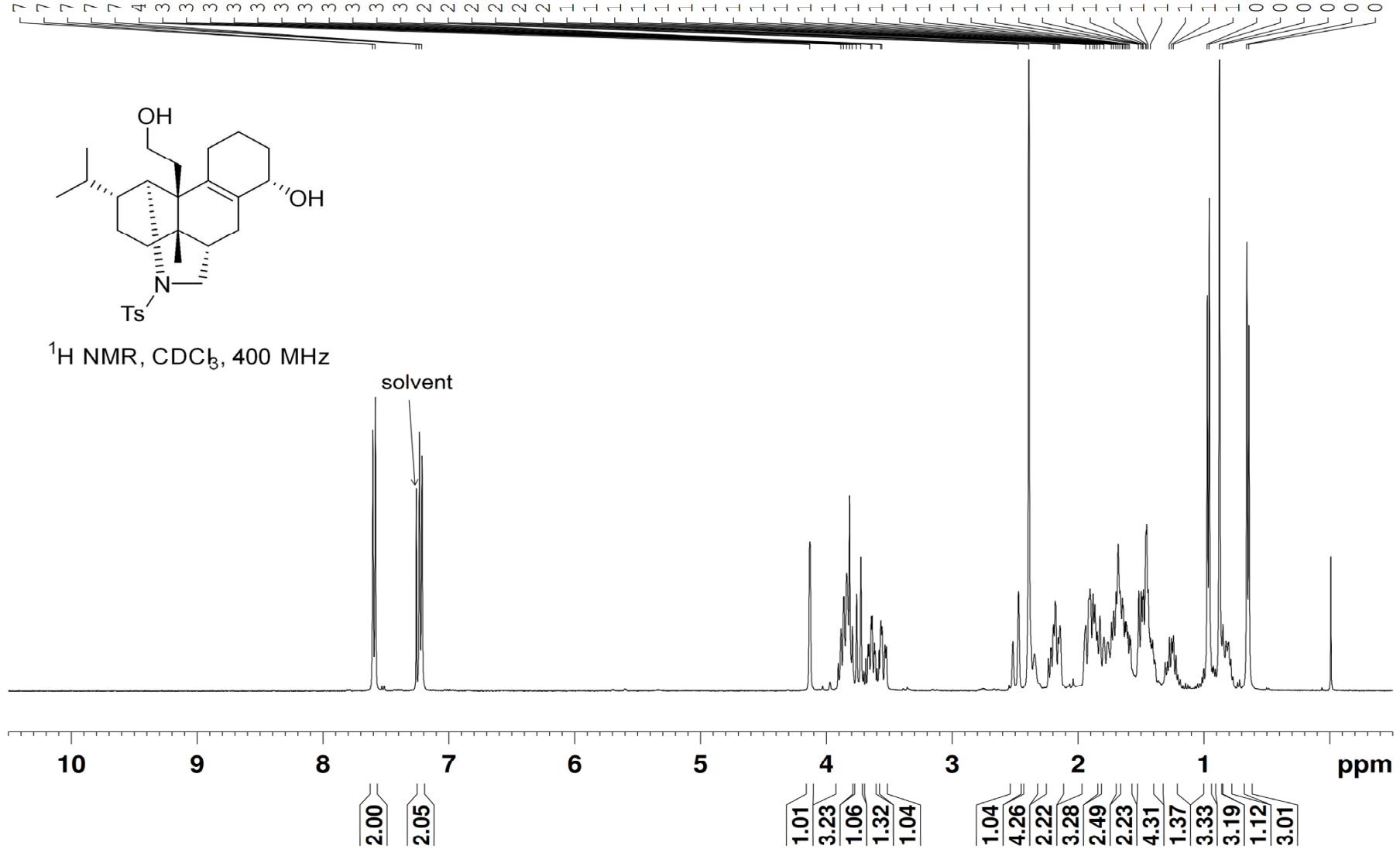


${ }^{13} \mathrm{C}$ NMR of 29

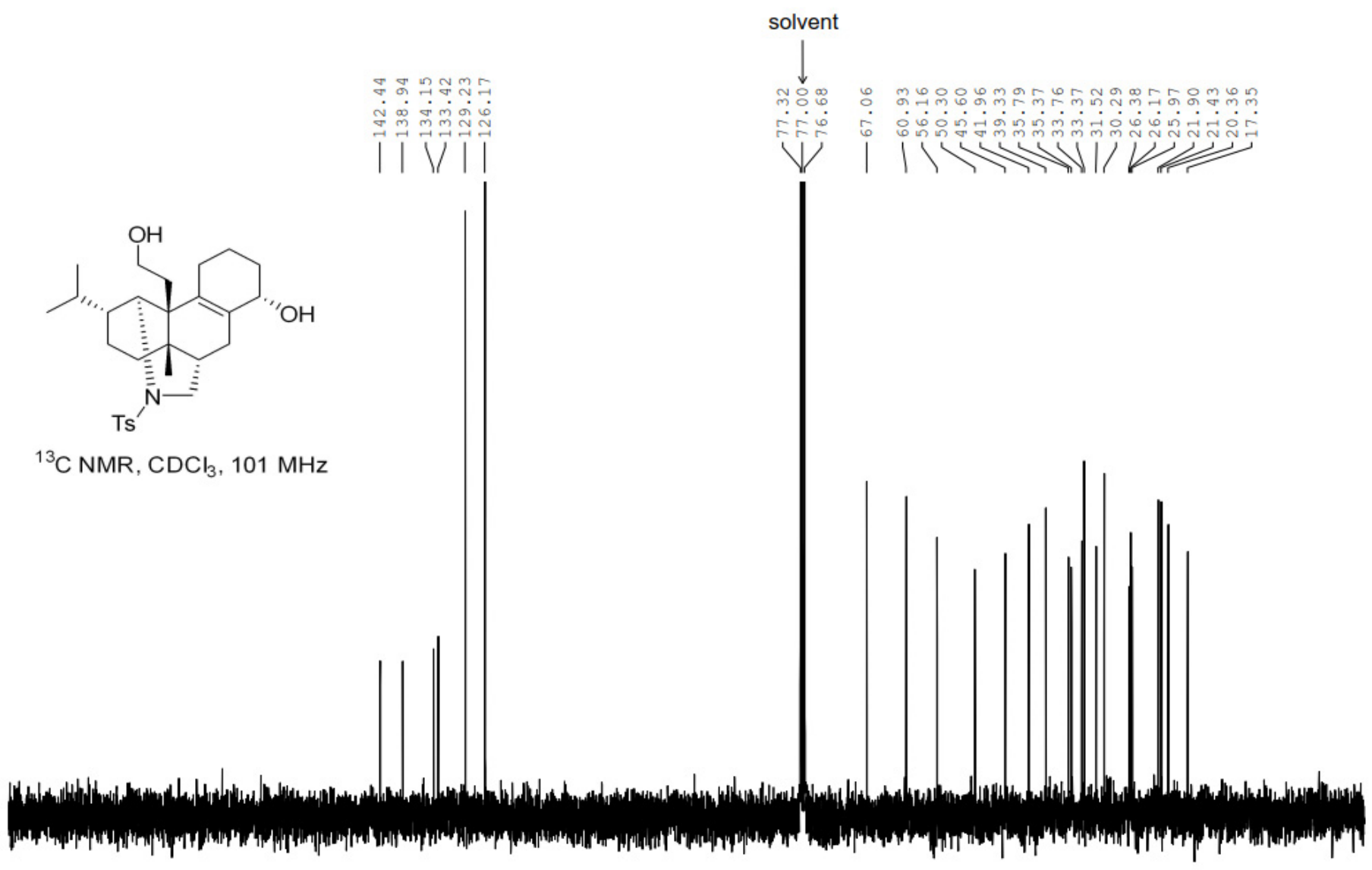

$\begin{array}{llllllllllllllllllll}190 & 180 & 170 & 160 & 150 & 140 & 130 & 120 & 110 & 100 & 90 & 80 & 70 & 60 & 50 & 40 & 30 & 20 & 10 & \text { ppm }\end{array}$ 


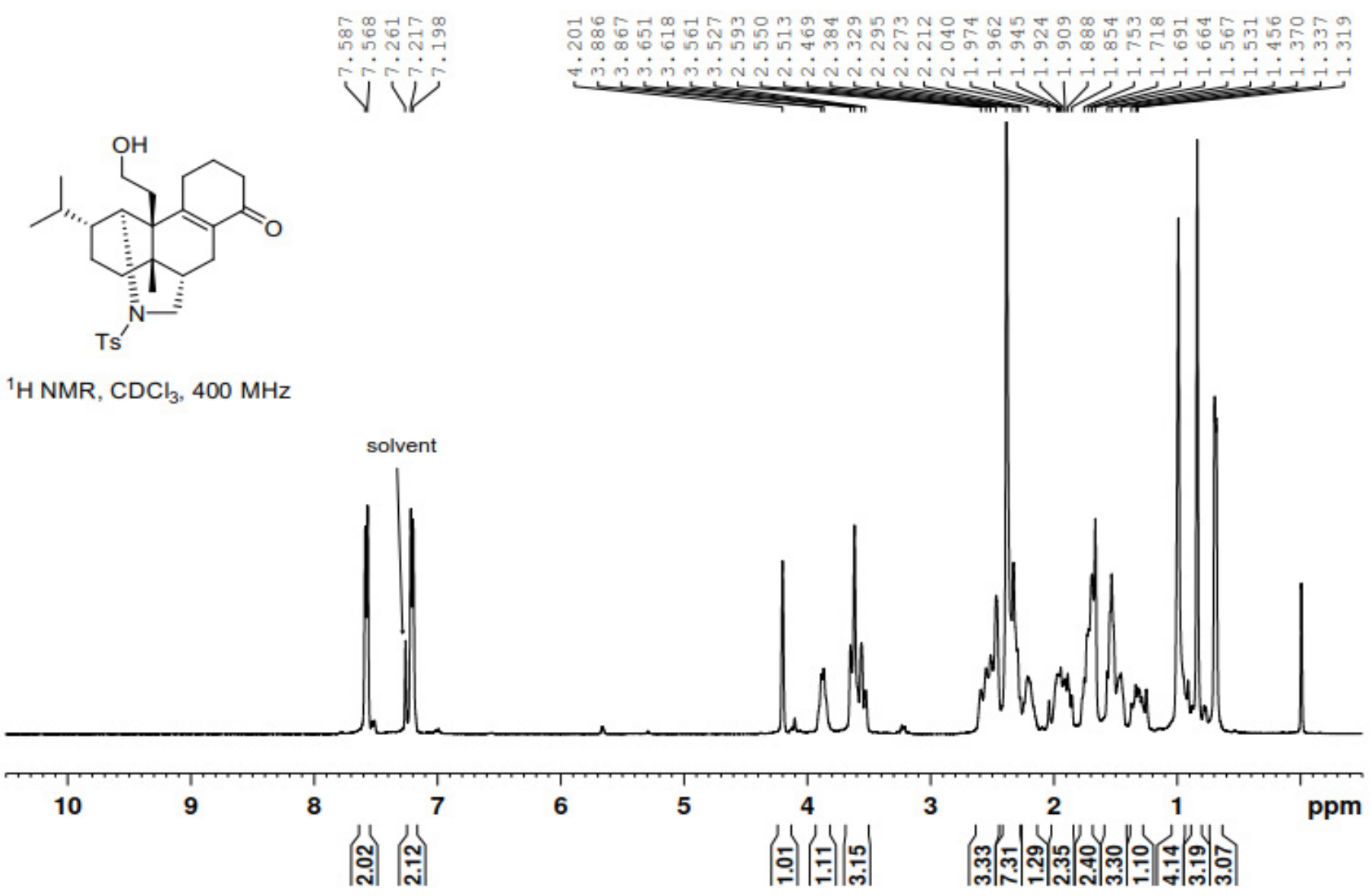


${ }^{13} \mathrm{C}$ NMR of 30

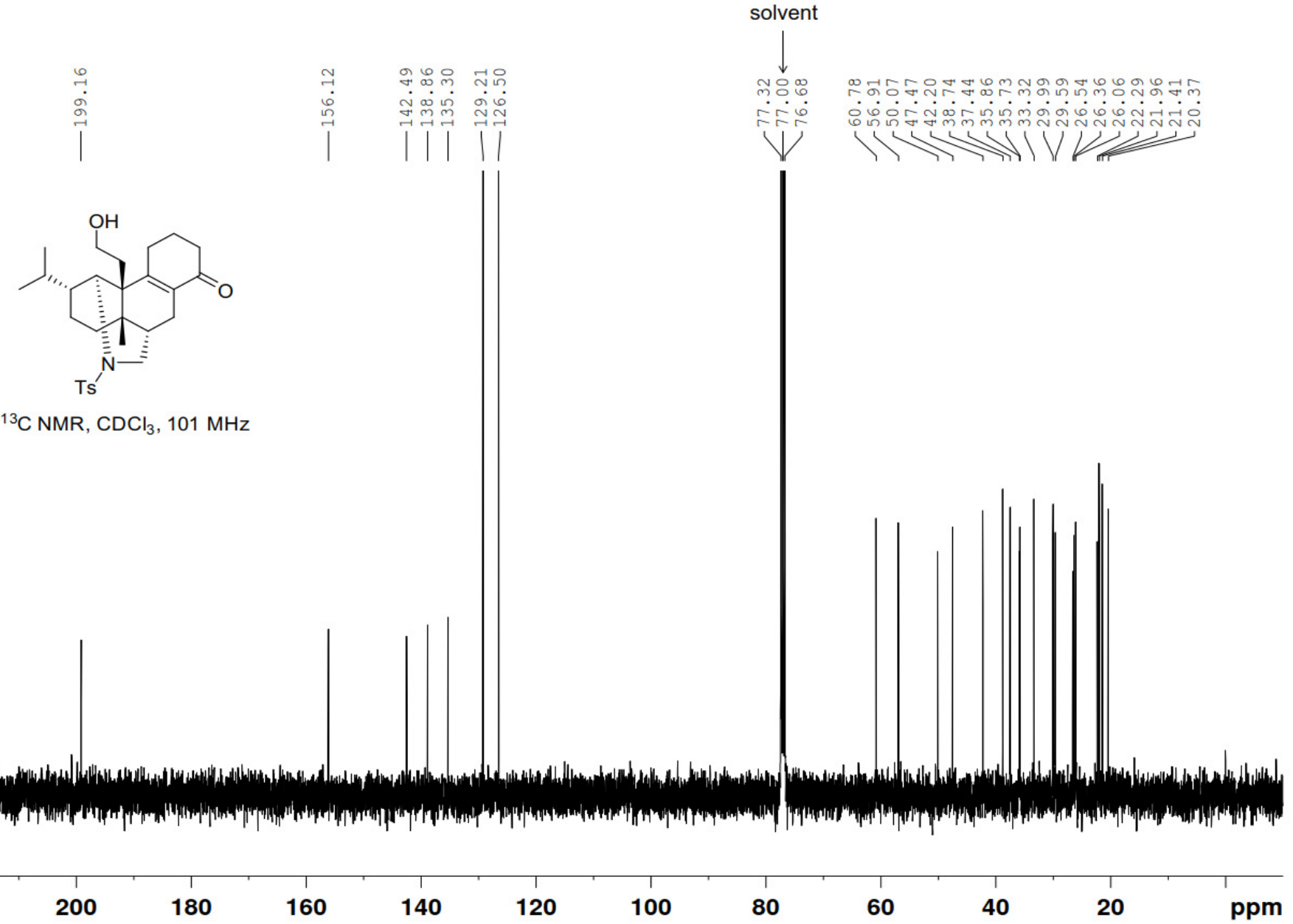




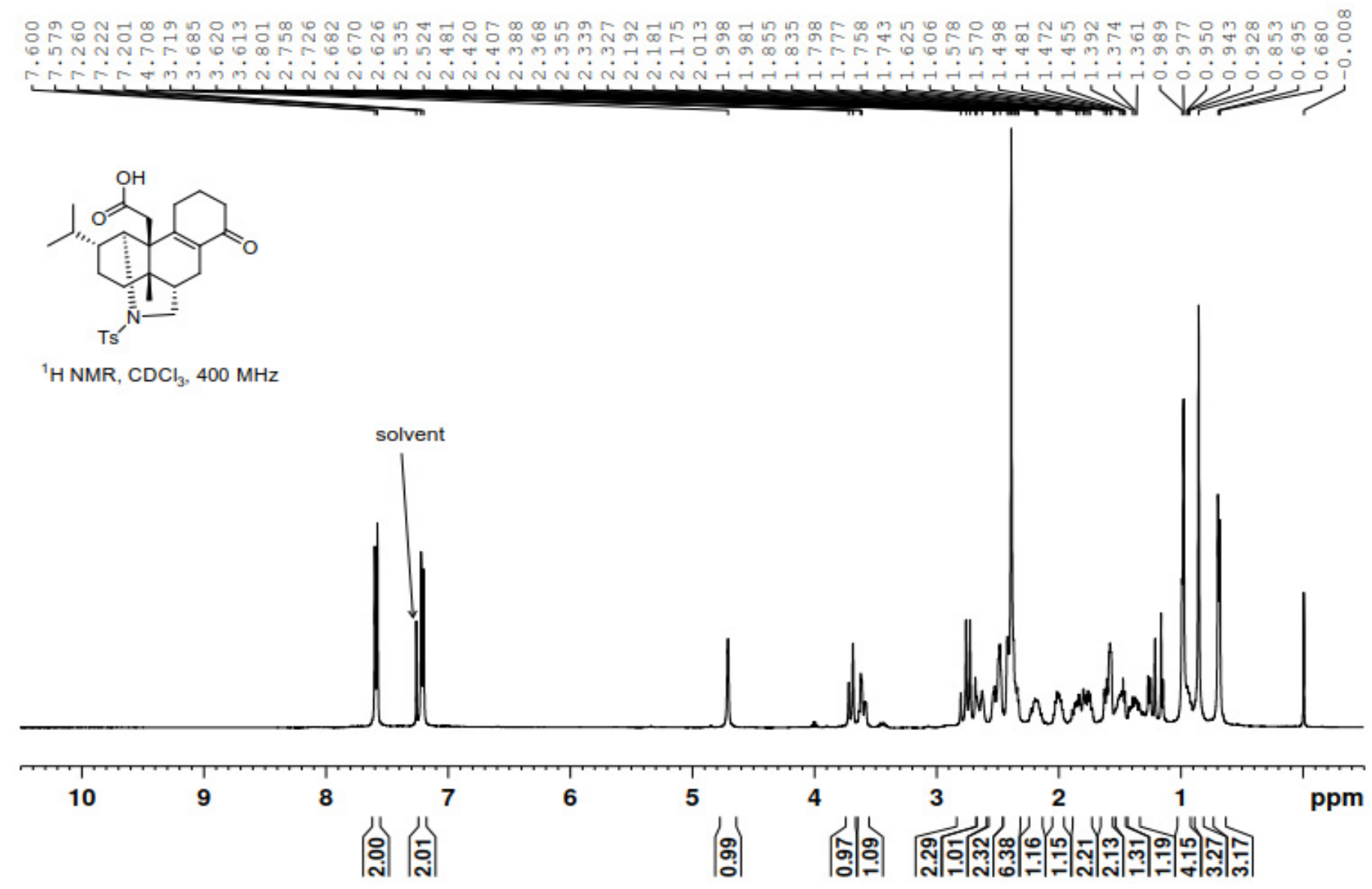


${ }^{13} \mathrm{C}$ NMR of 32

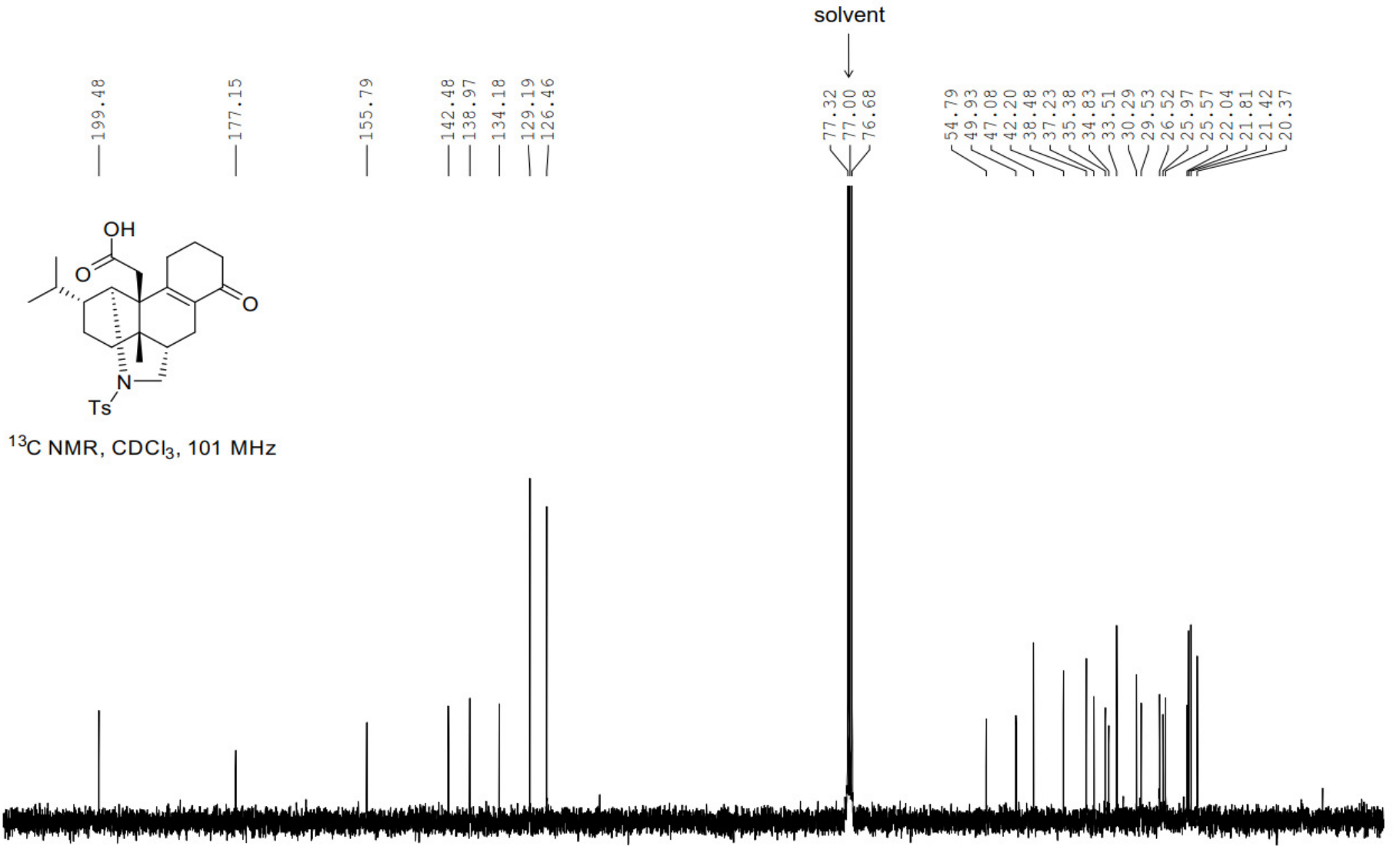

$200 \quad 180$ 160 140 120 100 80 60 


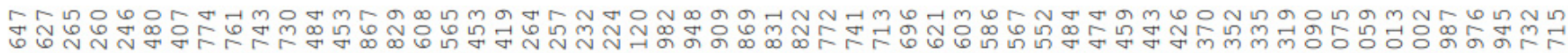
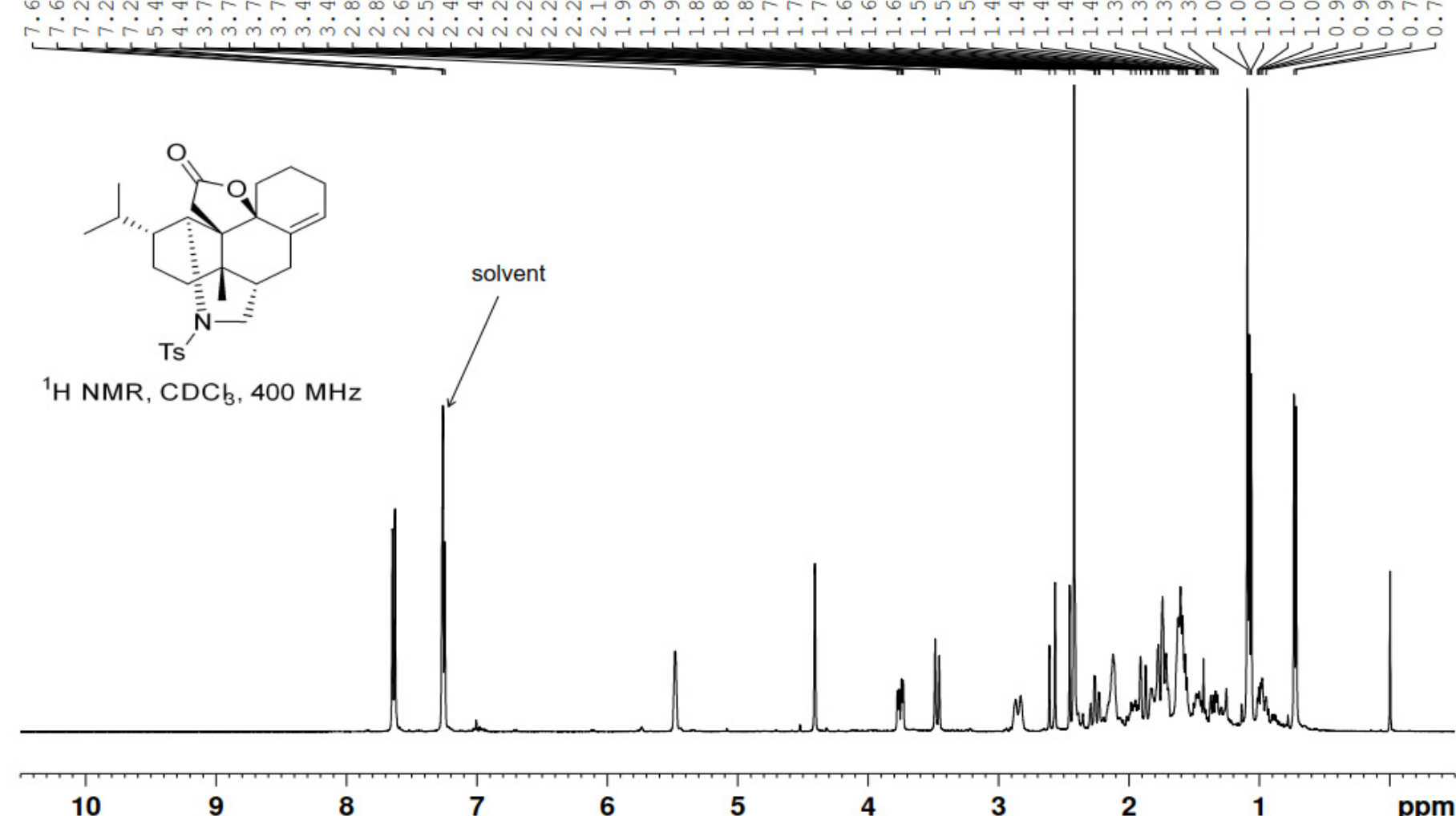

8

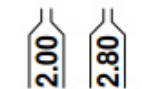
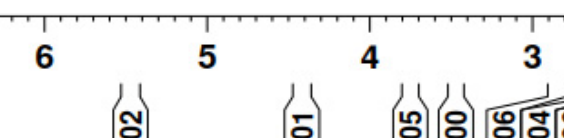
2 $1 \quad$ ppm

|하 | 
${ }^{13} \mathrm{C}$ NMR of 36
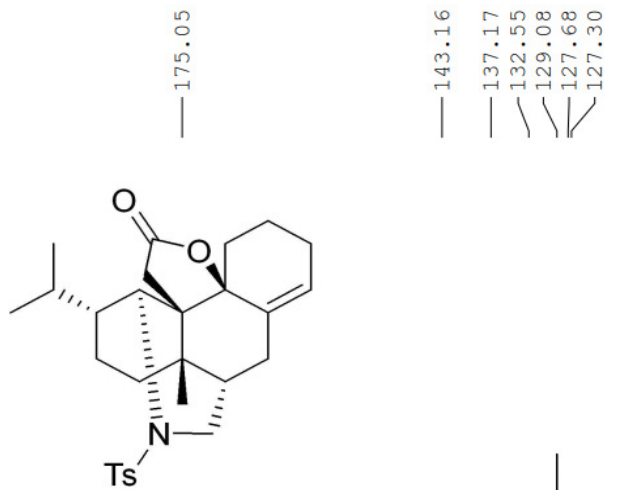

solvent

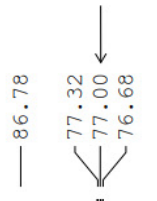

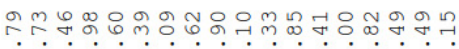

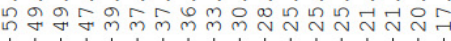

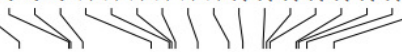

Ts

${ }^{13} \mathrm{C} \mathrm{NMR}, \mathrm{CDCl}_{3}, 101 \mathrm{MHz}$

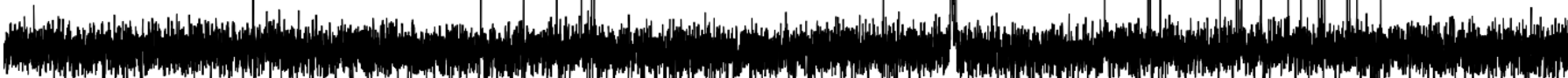
200
180
160
140
120
100
80
60
40
20
ppm 


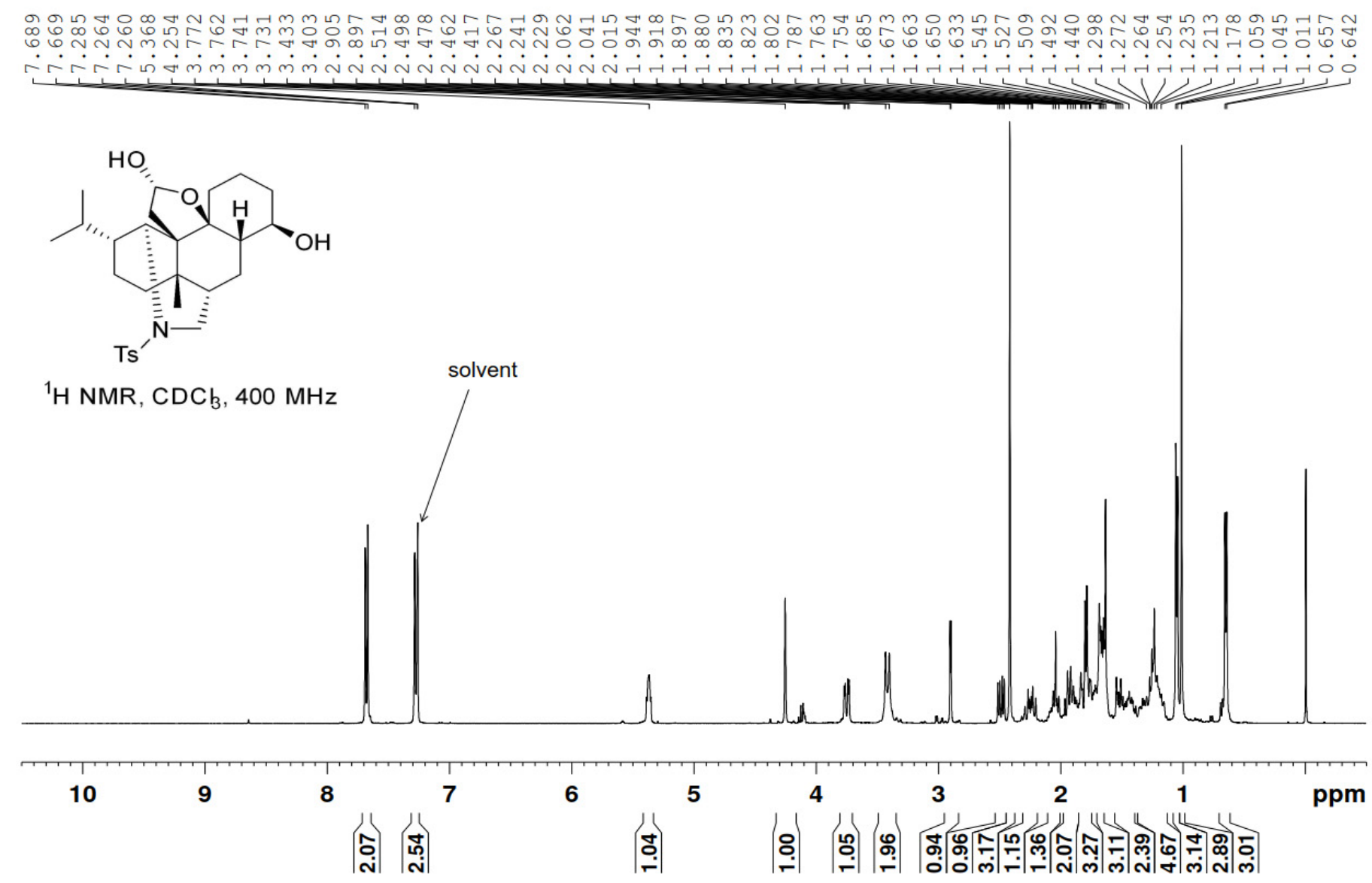


${ }^{13} \mathrm{C}$ NMR of 37

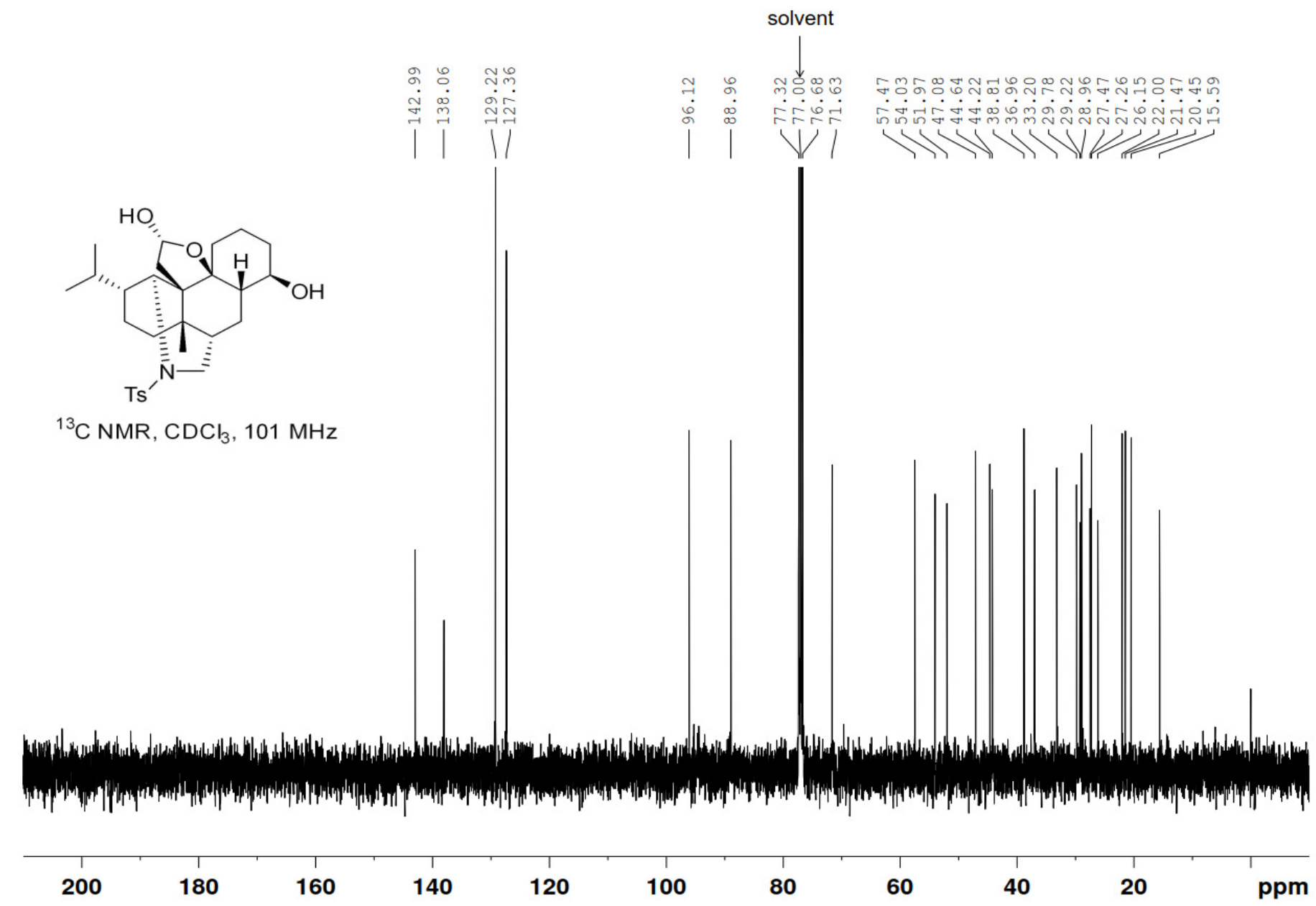



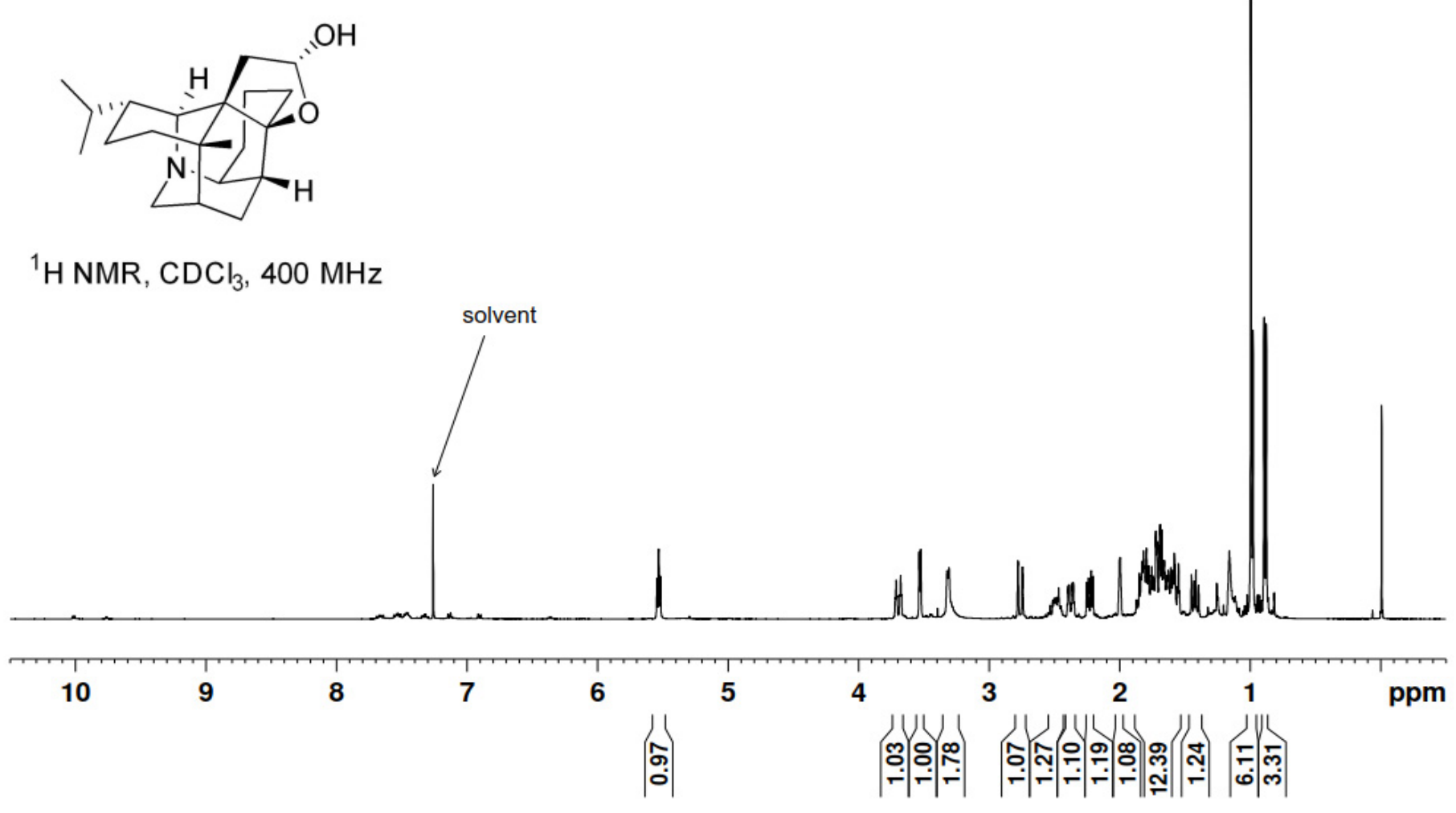
${ }^{13} \mathrm{C}$ NMR of 38
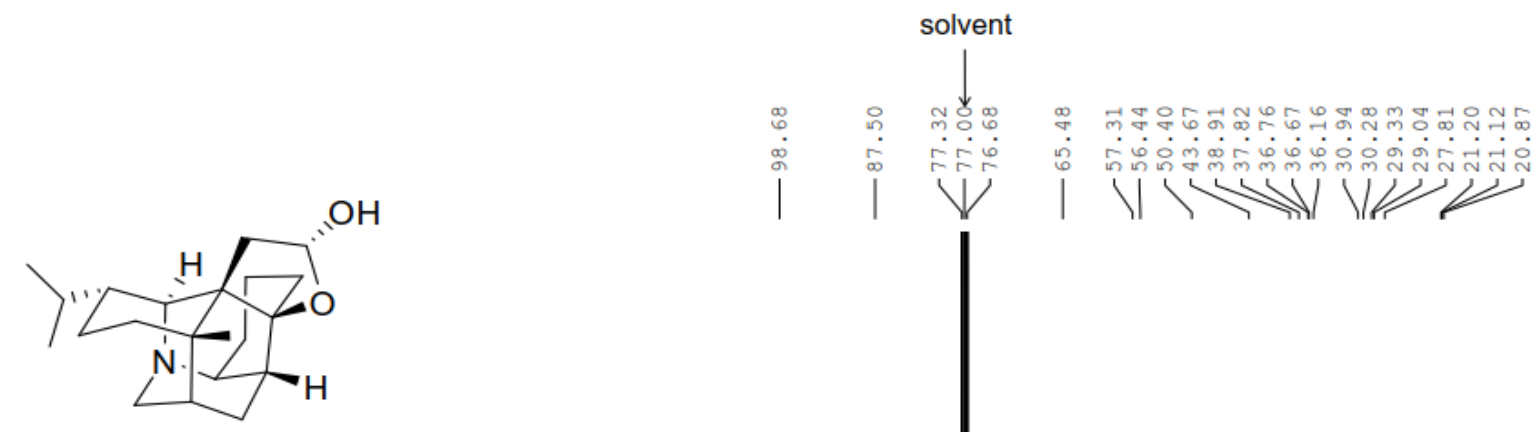

${ }^{13} \mathrm{C} \mathrm{NMR,} \mathrm{CDCl}_{3}, 101 \mathrm{MHz}$

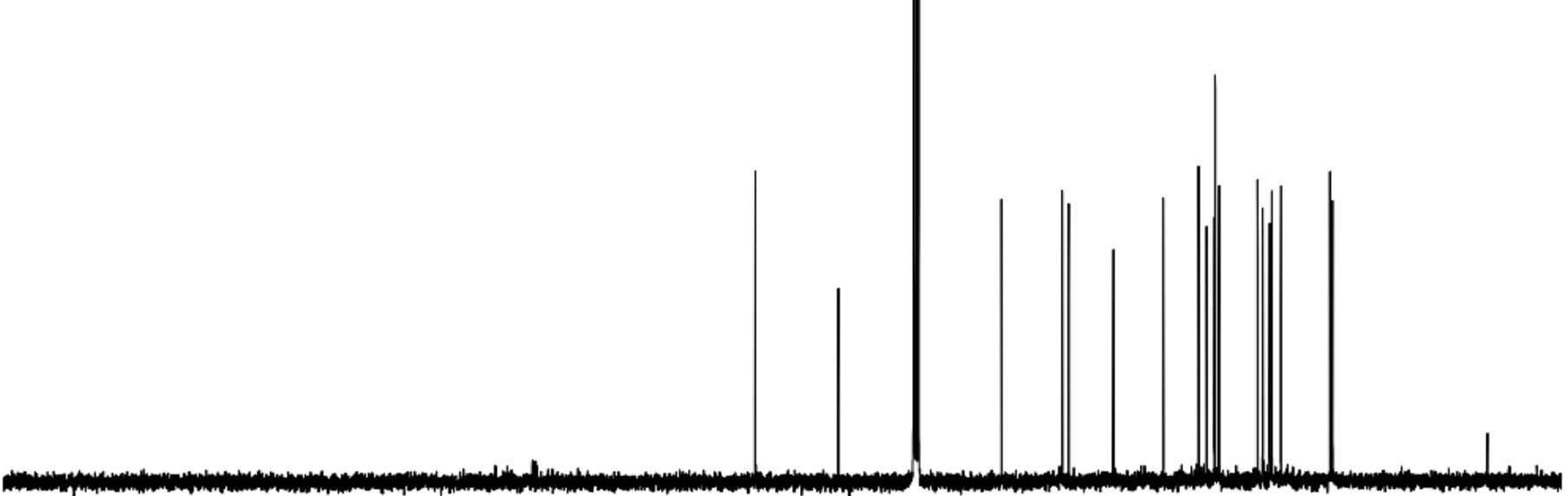

$\begin{array}{llllllllllllllllllll}190 & 180 & 170 & 160 & 150 & 140 & 130 & 120 & 110 & 100 & 90 & 80 & 70 & 60 & 50 & 40 & 30 & 20 & 10 & \mathrm{ppm}\end{array}$ 
${ }^{1} \mathrm{H}$ NMR of $1(\mathrm{pH}=7.0-7.5)$

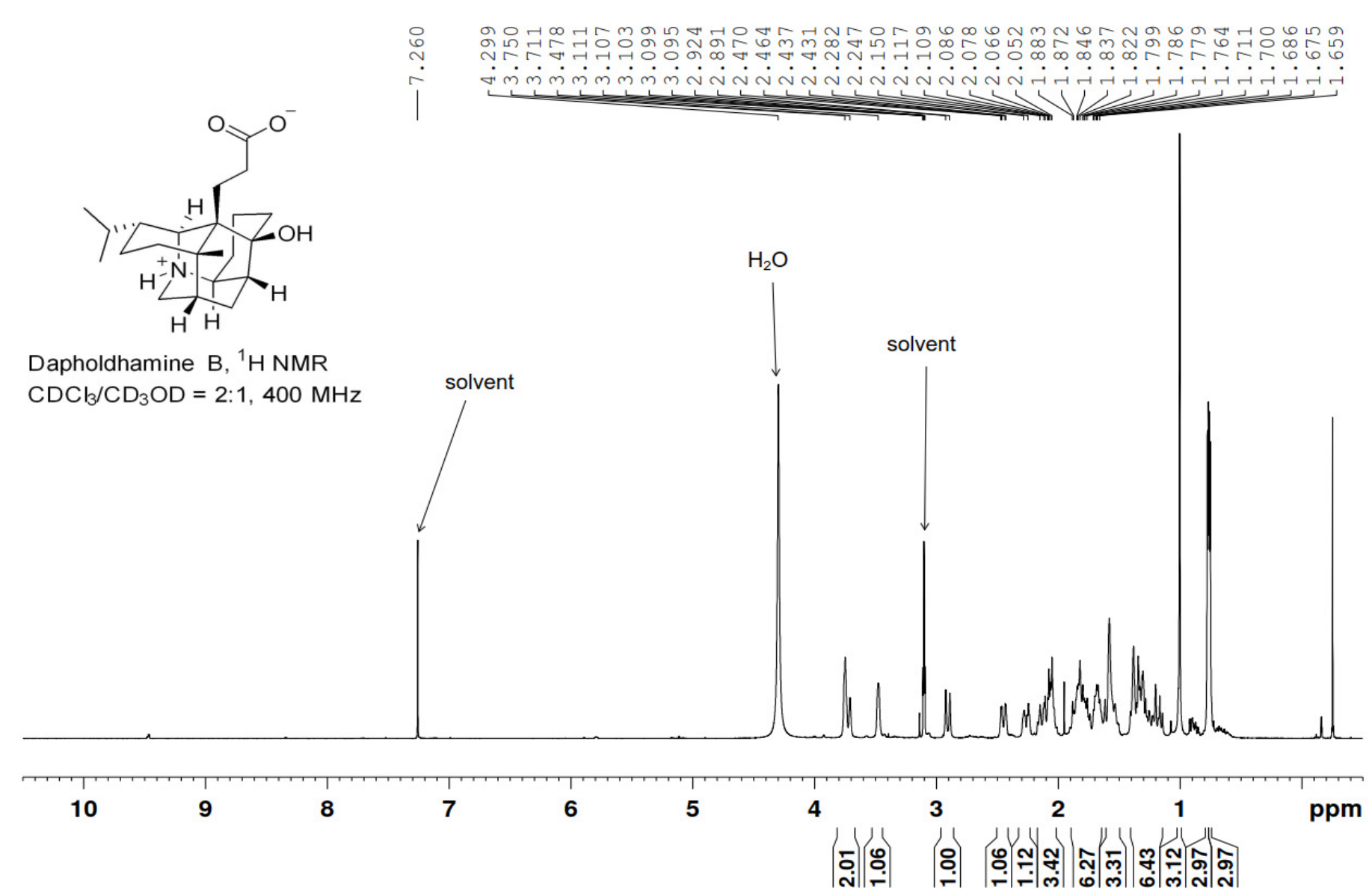


${ }^{13} \mathrm{C} \mathrm{NMR}$ of $\mathbf{1}(\mathrm{pH}=7.0-7.5)$

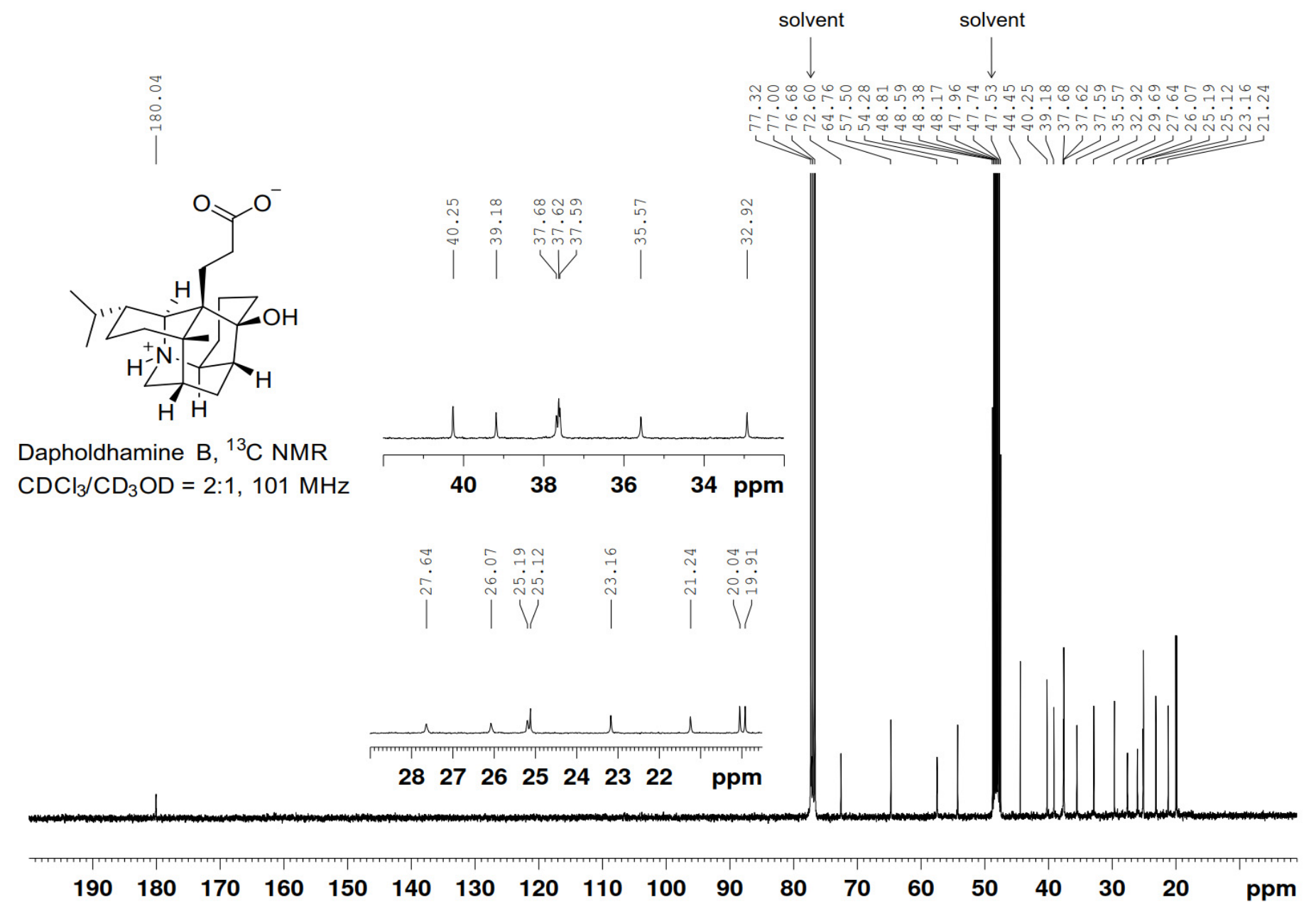


${ }^{13} \mathrm{C}$ DEPT135 NMR of $\mathbf{1}(\mathrm{pH}=7.0-7.5)$

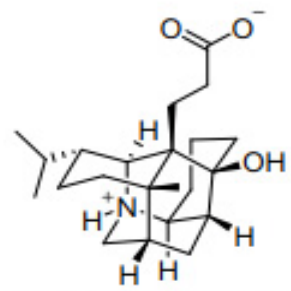

Dapholdhamine $\mathrm{B},{ }^{13} \mathrm{C}$ DEPT135 NMR $\mathrm{CDCl}_{3} / \mathrm{CD}_{3} \mathrm{OD}=2: 1,101 \mathrm{MHz}$

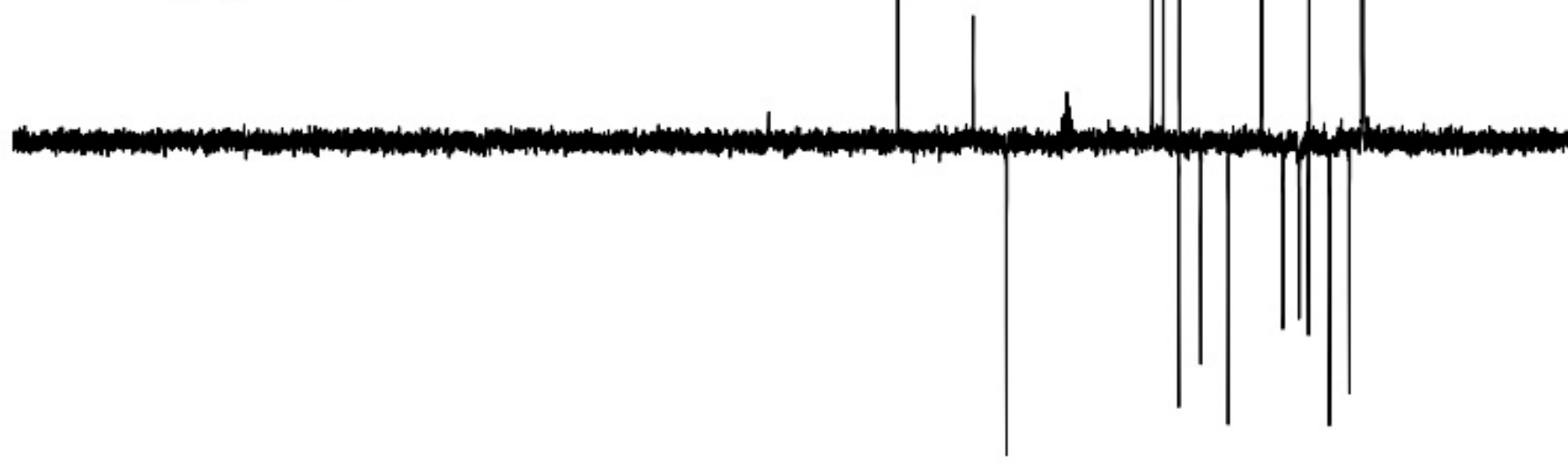

$\begin{array}{lllll}140 & 130 & 120 & 110 & 100\end{array}$

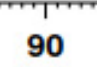

80

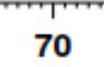

60
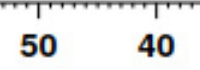

30

20

$10 \mathrm{ppm}$ 
${ }^{13} \mathrm{C}$ and ${ }^{13} \mathrm{C}$ DEPT135 NMR of $\mathbf{1}(\mathrm{pH}=7.0-7.5)$

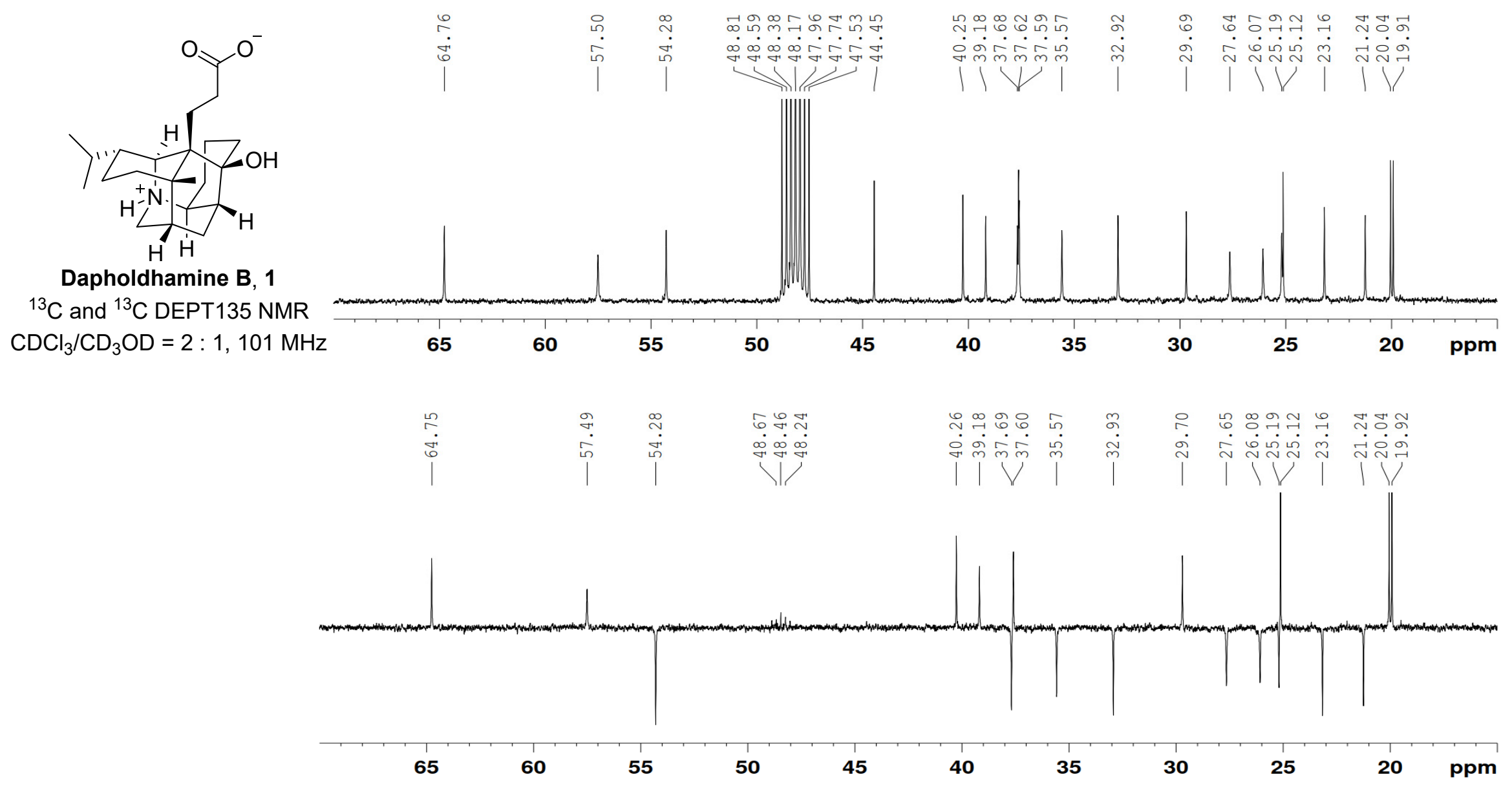




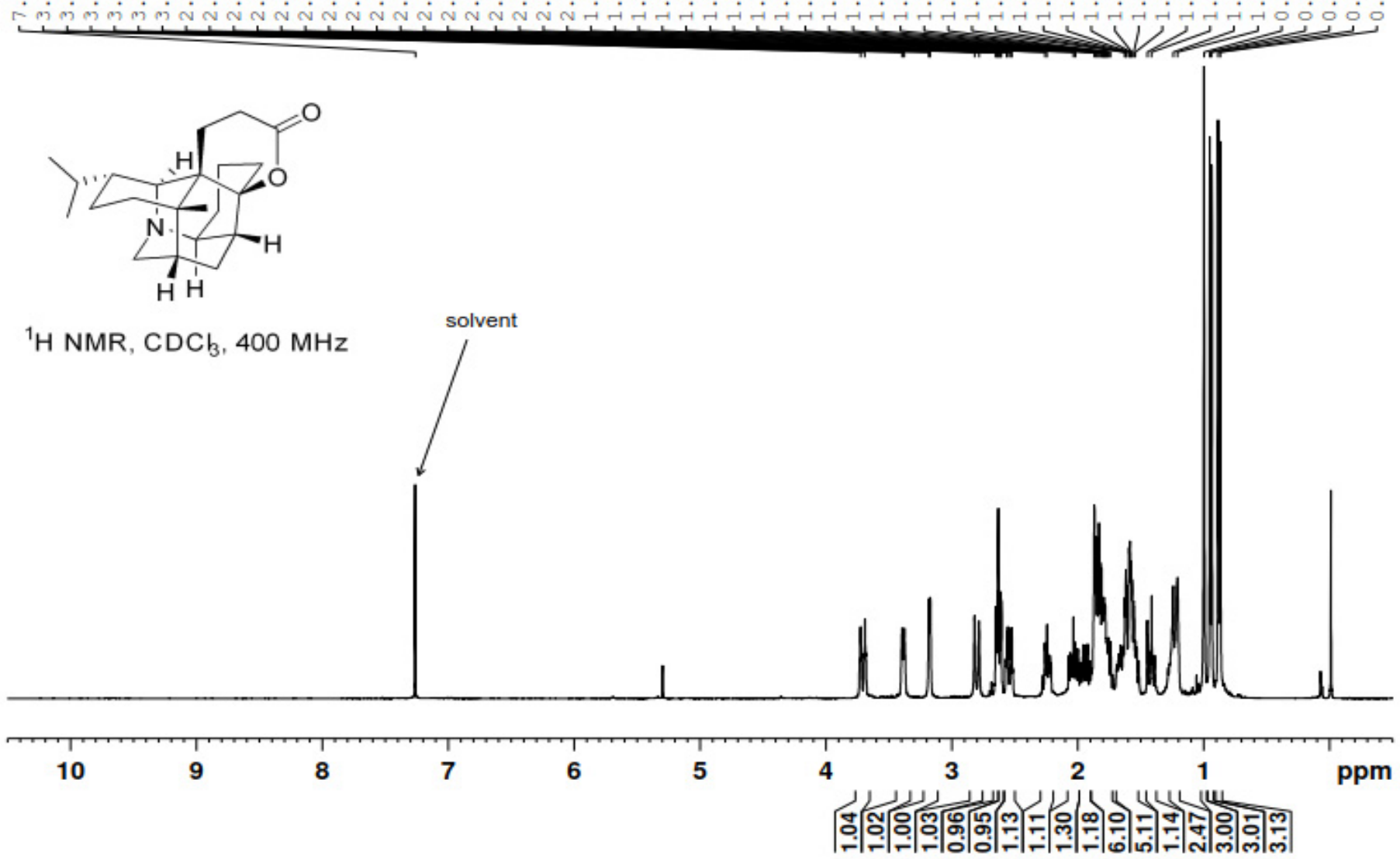


${ }^{13} \mathrm{C}$ NMR of 42

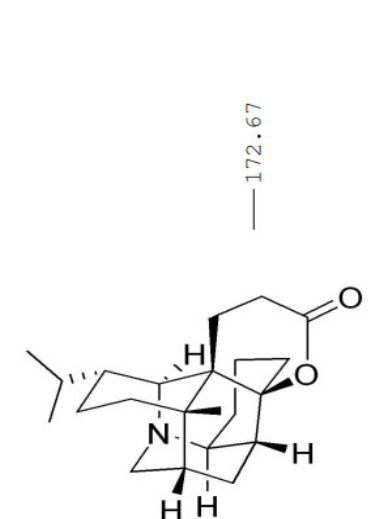

solvent

${ }^{13} \mathrm{C} \mathrm{NMR}, \mathrm{CDC}_{3}, 101 \mathrm{MHz}$

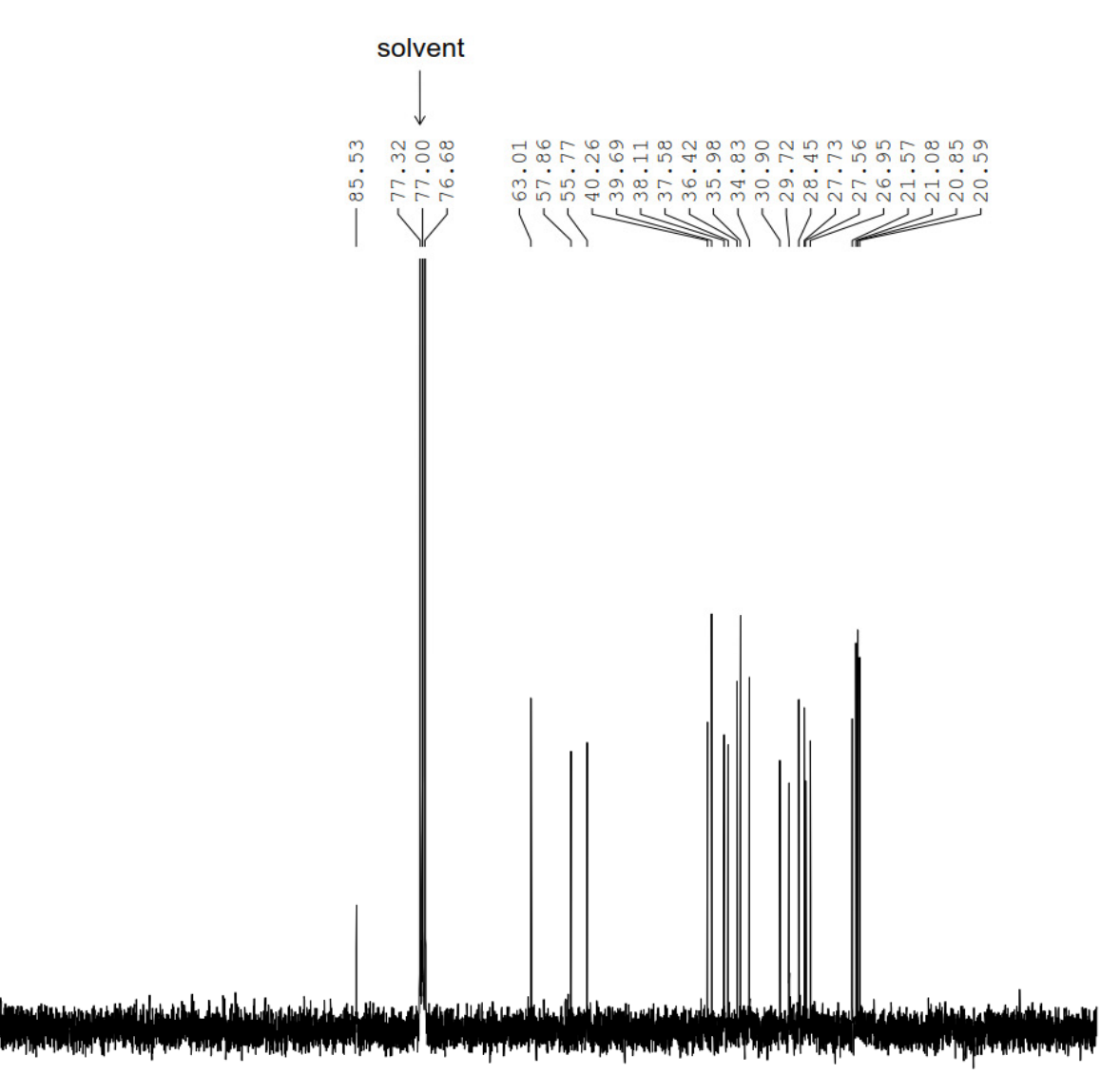

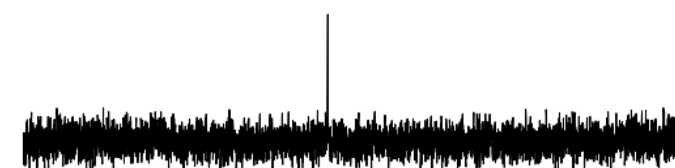

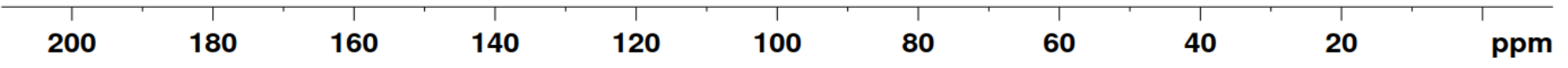


${ }^{13} \mathrm{C}$ DEPT135 NMR of 42
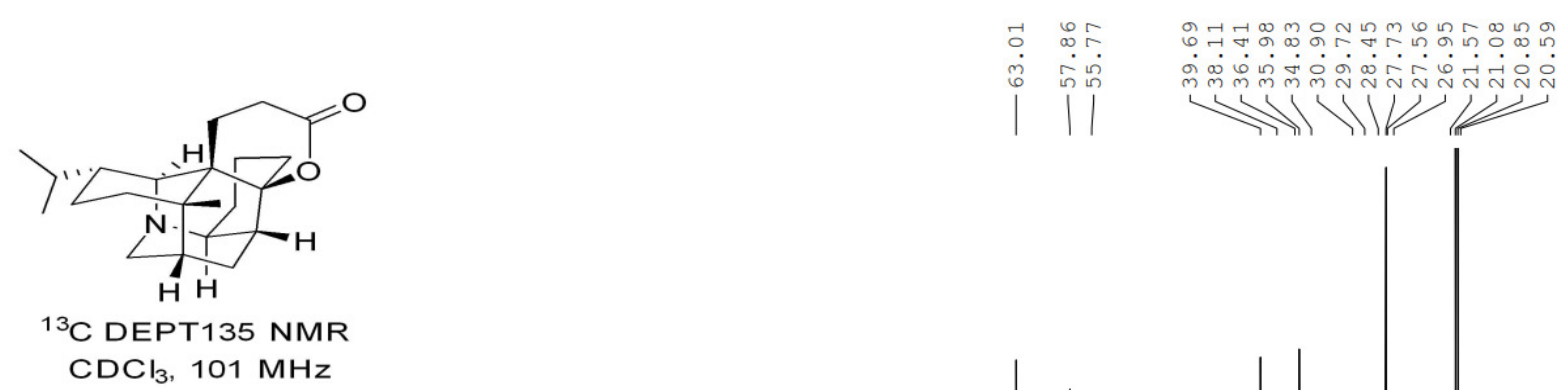

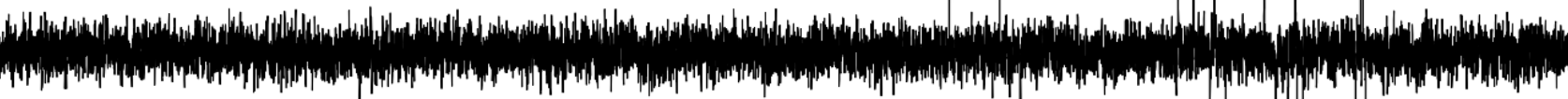

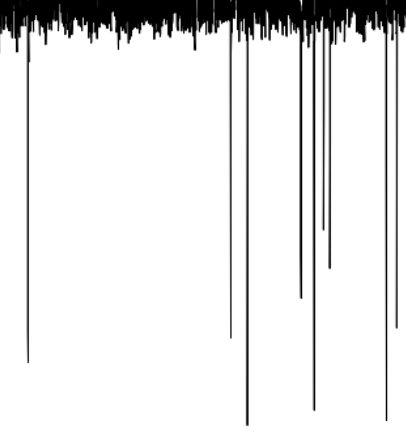
150
140130
120
110
100
90
80
70
0
40
30
20
10
0 ppm 
${ }^{1} \mathrm{H}-{ }^{1} \mathrm{H}$ COSY NMR of $\mathbf{4 2}$

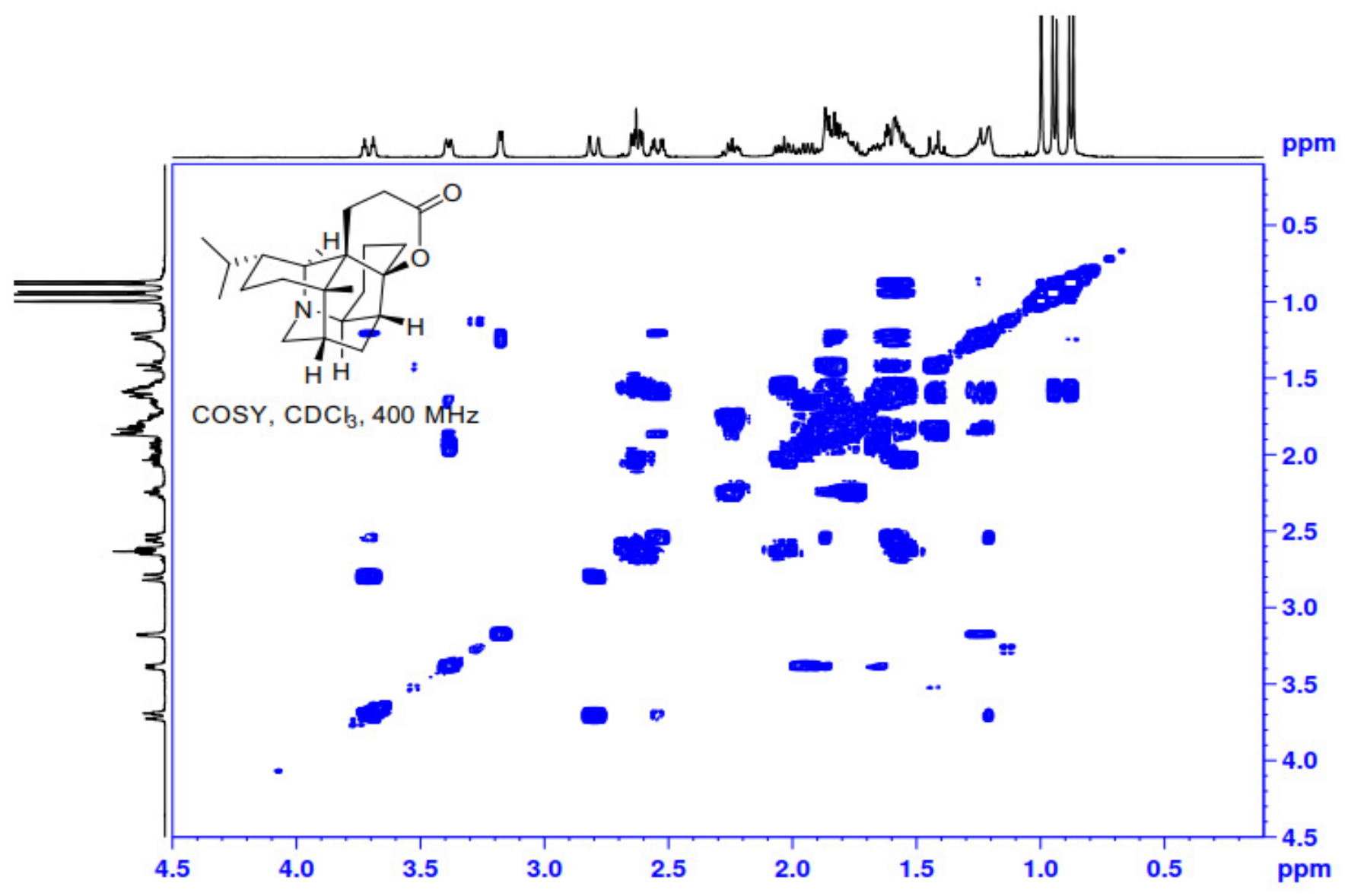


HSQC NMR of $\mathbf{4 2}$

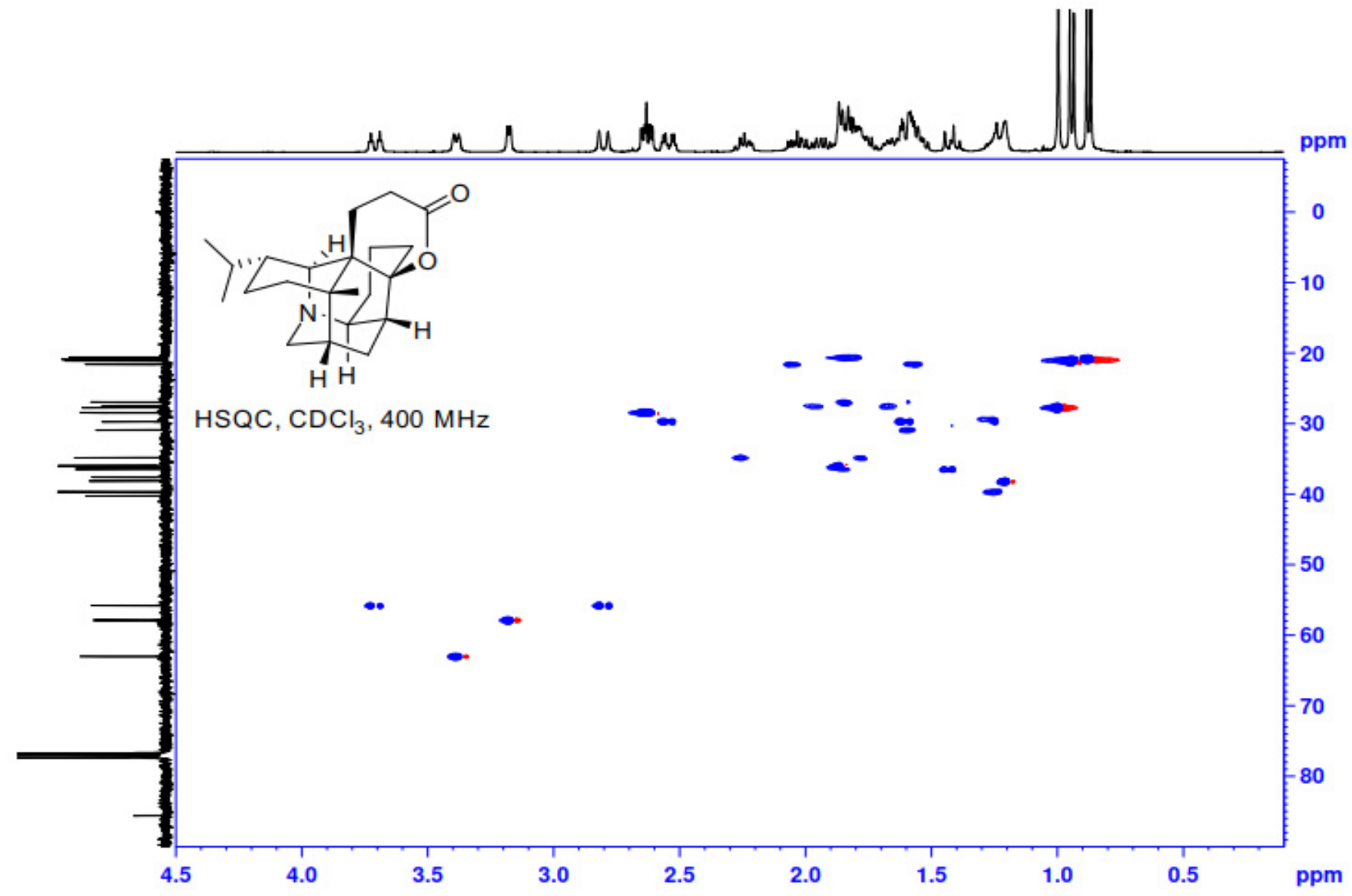


HMBC NMR of 42

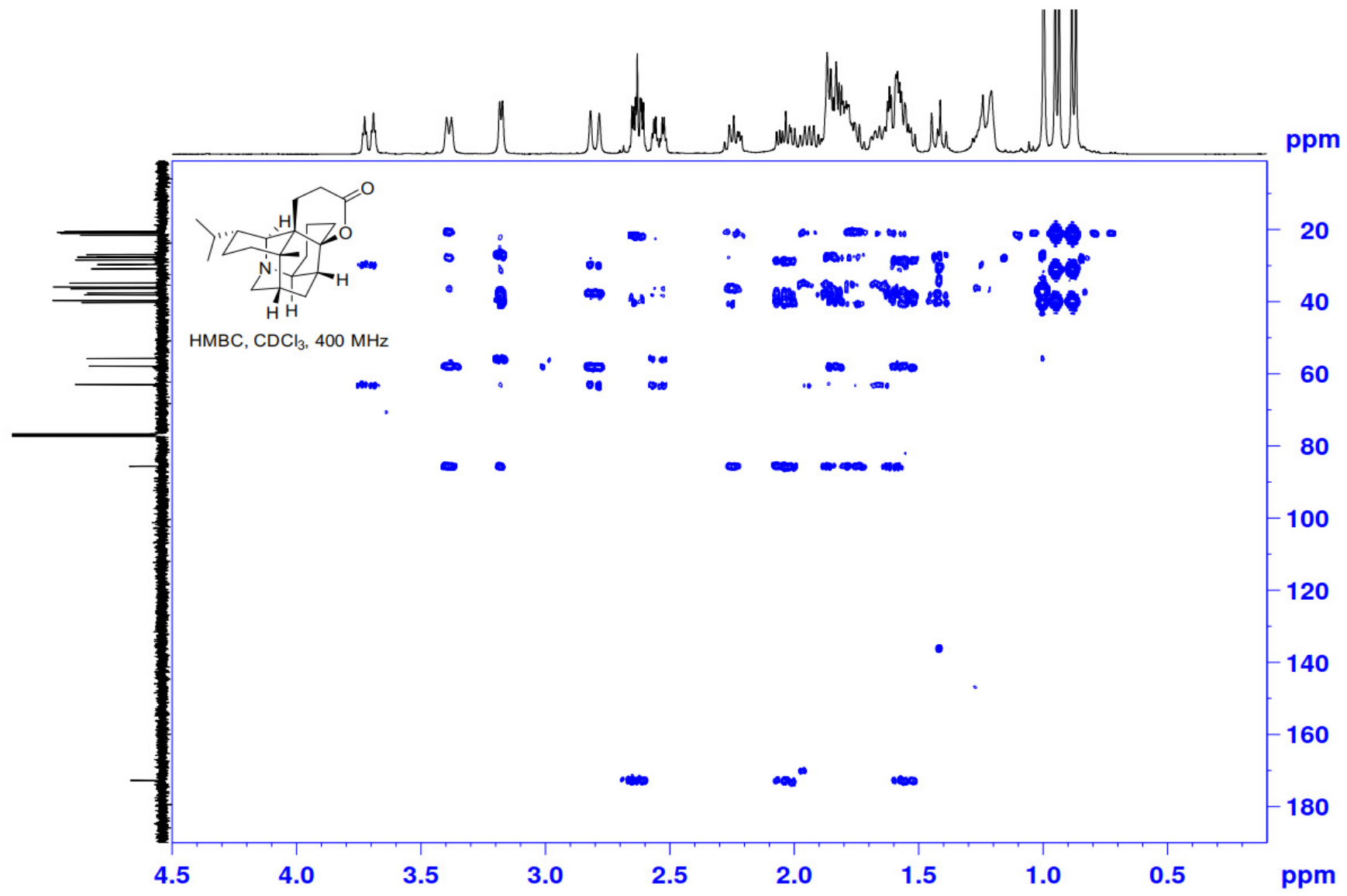




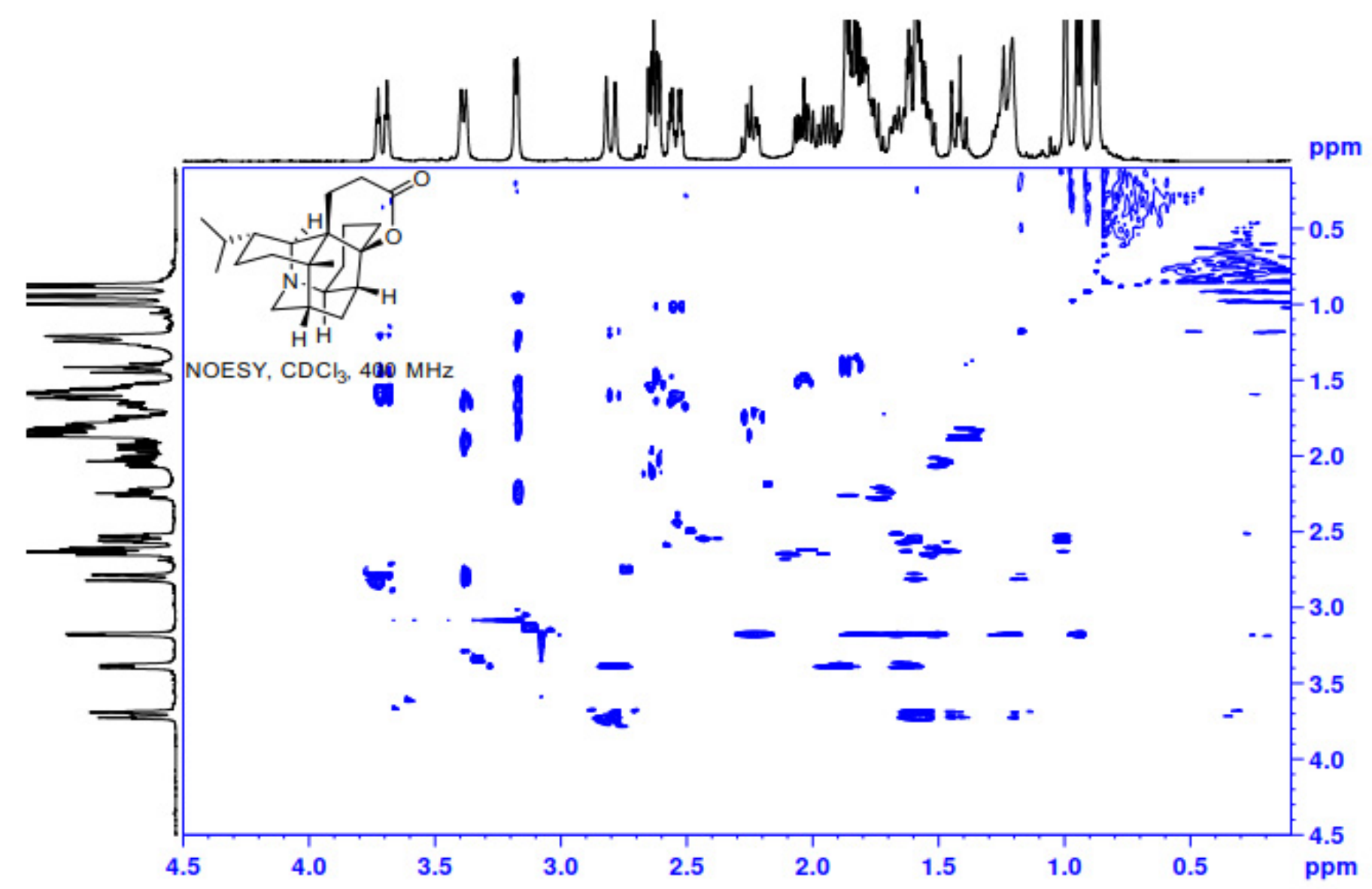

